\title{
Herbivore-induced plant volatiles and tritrophic interactions: from local to landscape scale
}
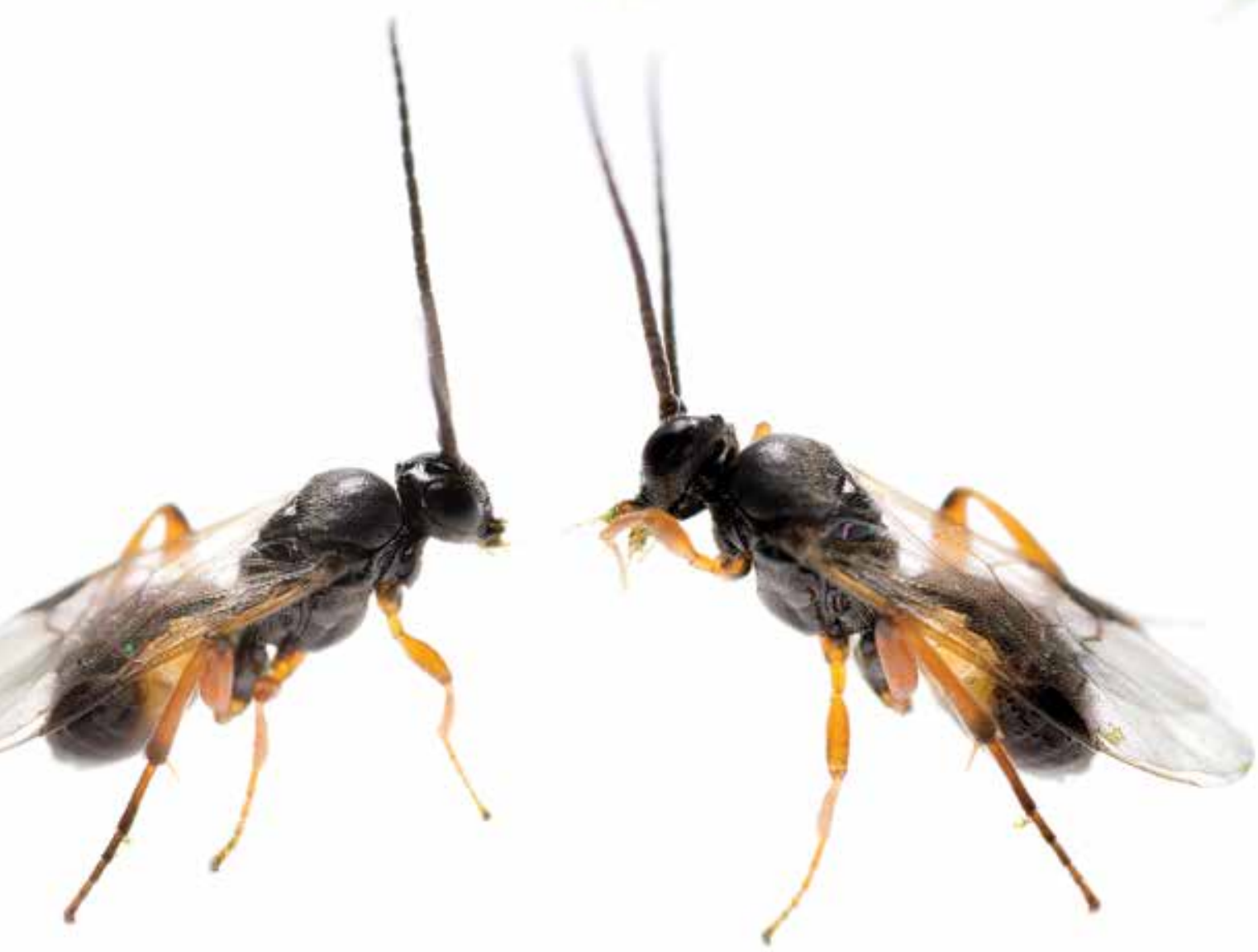

Yavanna Aartsma 


\section{Propositions}

1. To exploit crop varieties with enhanced parasitoid attraction for improving biological control, the surrounding landscape needs to be taken into account. (this thesis)

2. Taking movement capacity of insect species into account is important for studies of volatile-mediated insect-plant interactions.

(this thesis)

3. Scientists should stimulate science and curiosity at all levels of education.

4. Urbanisation not only affects natural ecosystems locally, but also globally by the extraction of construction materials.

5. Publishing papers is becoming more like online dating.

6. In the era of social media and digital manipulation, appreciation for non-manipulated nature is declining.

7. Every product should have full disclosure of its global supply chain.

Propositions belonging to the $\mathrm{PhD}$ thesis, entitled:

"Herbivore-induced plant volatiles and tritrophic interactions:

from local to landscape scale"

Yavanna Aartsma

Wageningen, 5 October 2018 


\title{
Herbivore-induced plant volatiles and tritrophic interactions: from local to landscape scale
}

\author{
Yavanna Aartsma
}




\section{Thesis committee}

\section{Promotor}

Prof. Dr Marcel Dicke

Professor of Entomology

Wageningen University \& Research

\section{Co-promotors}

Dr Felix J.J.A. Bianchi

Assistant Professor, Farming Systems Ecology Group

Wageningen University \& Research

Dr Erik H. Poelman

Associate Professor, Laboratory of Entomology

Wageningen University \& Research

Dr Wopke van der Werf

Associate Professor, Centre for Crop Systems Analysis

Wageningen University \& Research

\section{Other members}

Prof. Dr David Kleijn, Wageningen University \& Research

Dr Nina E. Fatouros, Wageningen University \& Research

Dr Olga Kostenko, Netherlands Institute of Ecology, Wageningen

Dr Tibor Bukovinszky, Koppert Biological Systems, Berkel en Rodenrijs

This research was conducted under the auspices of the C.T. de Wit

Graduate School for Production Ecology \& Resource Conservation. 


\title{
Herbivore-induced plant volatiles and tritrophic interactions: from local to landscape scale
}

\author{
Yavanna Aartsma
}

Thesis

submitted in fulfilment of the requirements for the degree of doctor at Wageningen University by the authority of the Rector Magnificus, Prof. Dr A.P.J. Mol, in the presence of the

Thesis Committee appointed by the Academic Board to be defended in public on Friday 5 October 2018 at 1.30 p.m. in the Aula. 
Yavanna Aartsma

Herbivore-induced plant volatiles and tritrophic interactions: from local to landscape scale 184 pages.

PhD thesis, Wageningen University, Wageningen, the Netherlands (2018) With references, with summary in English

ISBN: 978-94-6343-316-7

DOI: $10.18174 / 455955$ 



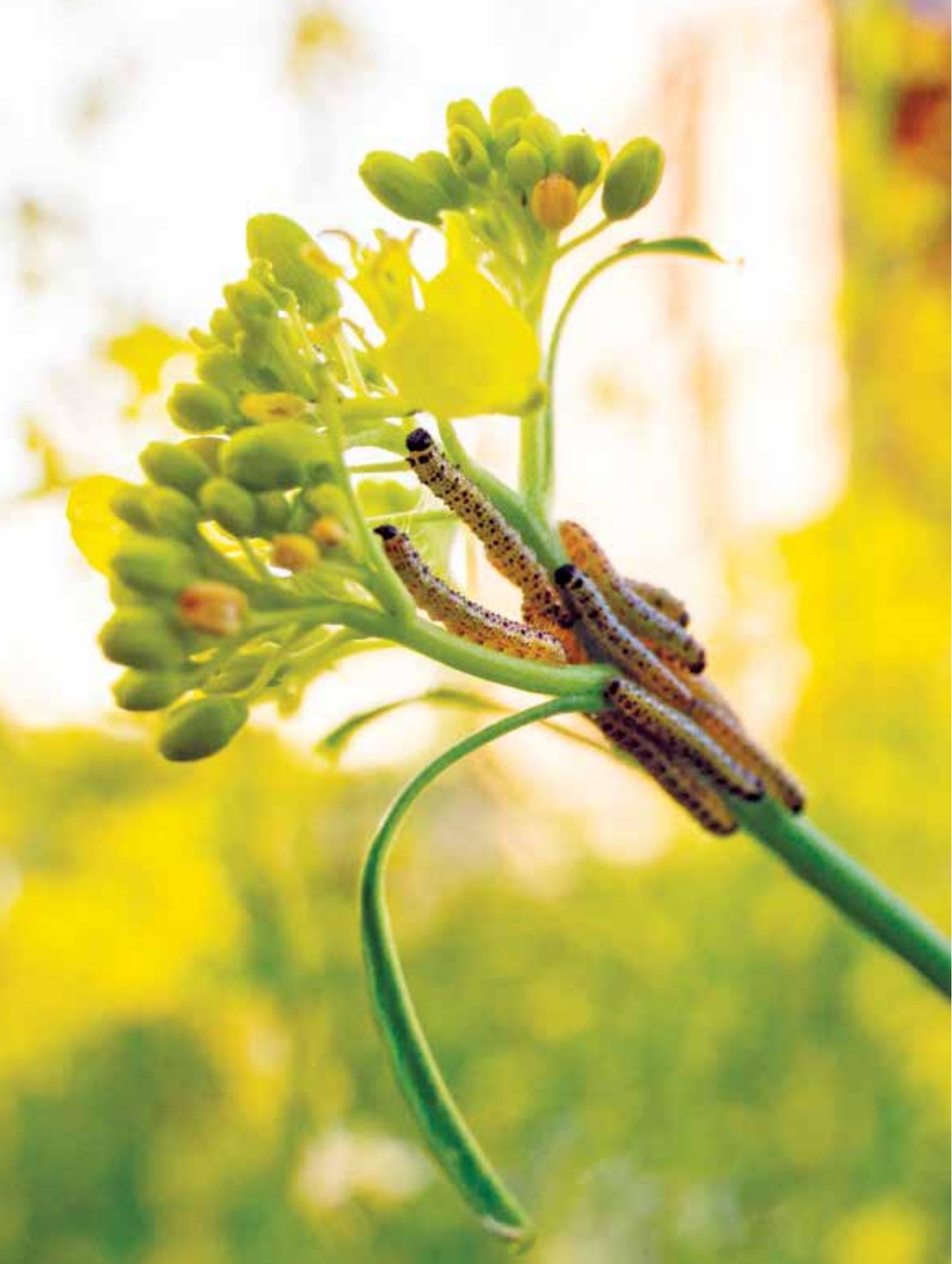




\section{Table of contents}

Chapter 1

General introduction

Chapter 2

Herbivore-induced plant volatiles and tritrophic interactions across spatial scales

\section{Chapter 3}

Intraspecific variation in herbivore-induced plant volatiles influences the spatial range of plant-parasitoid interactions

\section{Chapter 4}

Plant traits interact with habitat context in determining the strength of host-parasitoid relationships

\section{Chapter 5}

Herbivory by hosts and non-hosts, in interaction with plant traits, affects herbivore-induced plant volatiles and foraging efficiency of a specialis parasitoid

\section{Chapter 6}

Variation in herbivore-induced plant volatiles and alternative host plant cover mediate tritrophic interactions at the landscape scale

\section{Chapter 7}

General discussion

References

Summary

Acknowledgements

About the author

Publications

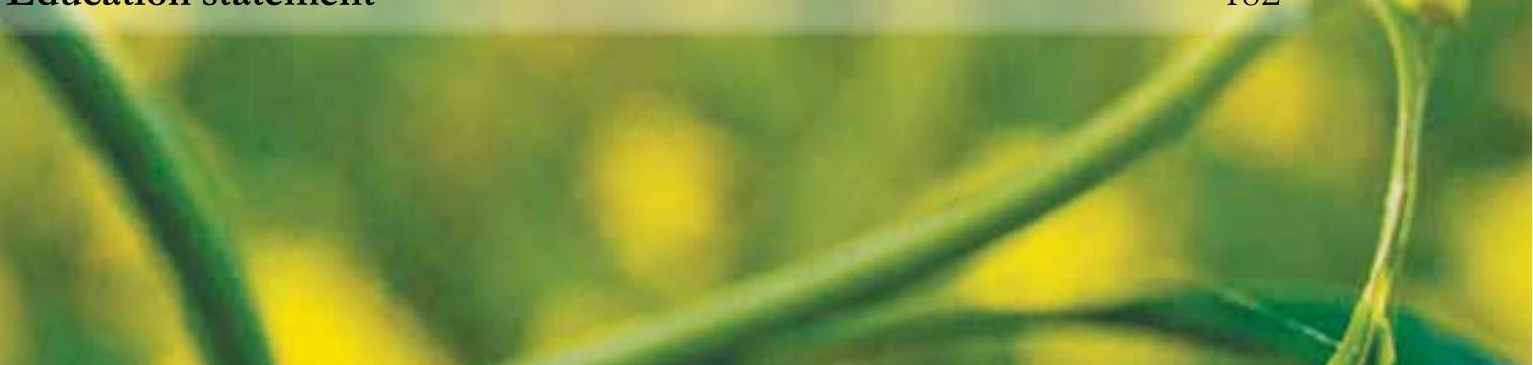




\section{Chapter 1}

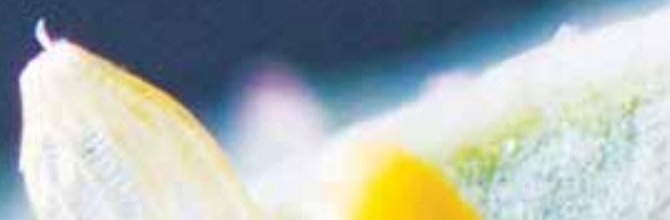




\section{General introduction}


Information has an important function in animal foraging behaviour and decision making (van Alphen et al., 2003; Wajnberg, 2006). Plants emit herbivore-induced plant volatiles (HIPVs) upon being exposed to herbivory, which can be used by natural enemies as information while they forage for prey or hosts (Dicke \& Baldwin, 2010; Hare, 2011). A number of elicitors can incite the release of HIPV from plants, varying from oral secretions to volatiles from other tissues of the same or neighbouring plants (Mattiacci et al., 1995; Engelberth et al., 2004). Because HIPVs can be produced systemically by plants, they are available in larger quantities and easier to detect than direct odour cues from individual herbivores (Vet \& Dicke, 1992). Blends of HIPVs can be affected by a wide range of factors such as the presence of multiple herbivores (de Rijk et al., 2013), abiotic stresses (Holopainen \& Gershenzon, 2010) or the developmental stage of the plant (Bruinsma et al., 2014). Such occurrences may impact the HIPVs to the extent that they become less reliable in conveying a specific herbivore's location (Vet \& Dicke, 1992). After leaving the plant, HIPVs also become subject to environmental factors such as atmospheric conditions and the presence of other volatiles in the air (Blande et al., 2014). As a result, natural enemies are searching for hosts in a spatially and temporally dynamic, heterogeneous environment. The emission of HIPVs and other volatiles by multiple sources in interaction with environmental factors, such as wind and turbulence, results in a 'volatile mosaic', and may provide a mechanistic understanding of an important driver of movement by natural enemies (Aartsma et al., 2017).

While the importance of HIPVs as chemical cues for parasitoids and predators is widely recognised, the spatial scale at which they influence foraging decisions remains an open question (Heil, 2014; Schellhorn et al., 2014). Furthermore, while it is known that natural enemy foraging efficiency can depend on the context in which HIPV-mediated tritrophic interactions takes place, little is known on how plant traits - such as attractiveness to natural enemies via HIPVs - affect natural enemy foraging decisions in the field. At what spatial scales do HIPVs affect natural enemy foraging and how does this depend on habitat characteristics? 
Parasitic wasps (parasitoids) are ubiquitous in natural and agricultural habitats, and parasitoid species constitute most of the total number of species in the order Hymenoptera, which might be considered the most speciose animal order (Forbes et al., 2018). Parasitoids lay eggs in, on or nearby other insects and during their development their larvae feed from a single host insect, eventually killing the host (Vinson, 1976; Godfray, 1994). Locating a host is therefore of vital importance for parasitoids, but female parasitoids emerging from their pupa may often be located in a habitat where new hosts are not available nearby (Vinson, 1976). In order to ensure procreation, they need to disperse and colonise new habitats (Vinson, 1976). Parasitoids often specialise on a few host species and, due to the direct link between host-finding success and parasitoid fitness, they are expected to be strongly selected for optimizing their foraging strategies regarding these hosts (van Alphen et al., 2003; Thiel \& Hoffmeister, 2009). They are also known to use HIPVs as a means to locate herbivorous hosts (Vet \& Dicke, 1992; Geervliet et al., 1994). This makes them an excellent model system to study foraging behaviour in the context of HIPVs and the spatial scale at which these compounds are useful when it comes to locating a host.

\section{Research objectives}

The aim of this thesis project was to investigate HIPV-mediated interactions between plants, herbivores and parasitoids at the plant, field and landscape scale. I also investigated how plant variation in HIPV-mediated attractiveness to parasitoids alters parasitoid movement and host-finding success at multiple spatial scales. In practical terms, I used two plant accessions which are known to differ in attractiveness to parasitoids via HIPVs and also ensured that the special context in which parasitoids search for hosts on these plants varied. I specifically investigated the effects of plant distance, spatial context, presence of other herbivores and alternative host plant cover in the landscape. 


\section{Study system}

\section{Plant species/variety}

The plants used in this thesis belong to the species Brassica oleracea var alba L., more commonly known as white cabbage. It is a cultivated form of wild cabbage (Brassica oleracea var oleracea), which has been domesticated into many distinct varieties to be grown worldwide as vegetable crops. Two varieties of white cabbage have been used, the accession 'Badger Shipper' and 'Christmas Drumhead'. Christmas Drumhead is more attractive to the parasitoid Cotesia glomerata upon damage by Pieris spp caterpillars than Badger Shipper (Poelman et al., 2009b). Cabbage plants can be attacked by various herbivores, including specialists of brassicaceous plants such as Pieris brassicae.

\section{Herbivore species}

Pieris brassicae (Lepidoptera: Pieridae)(Large cabbage white butterfly) caterpillars are specialist herbivores of plants in the family Brassicaceae. In the Netherlands, the species produces two or three generations per year. The female butterfly lays
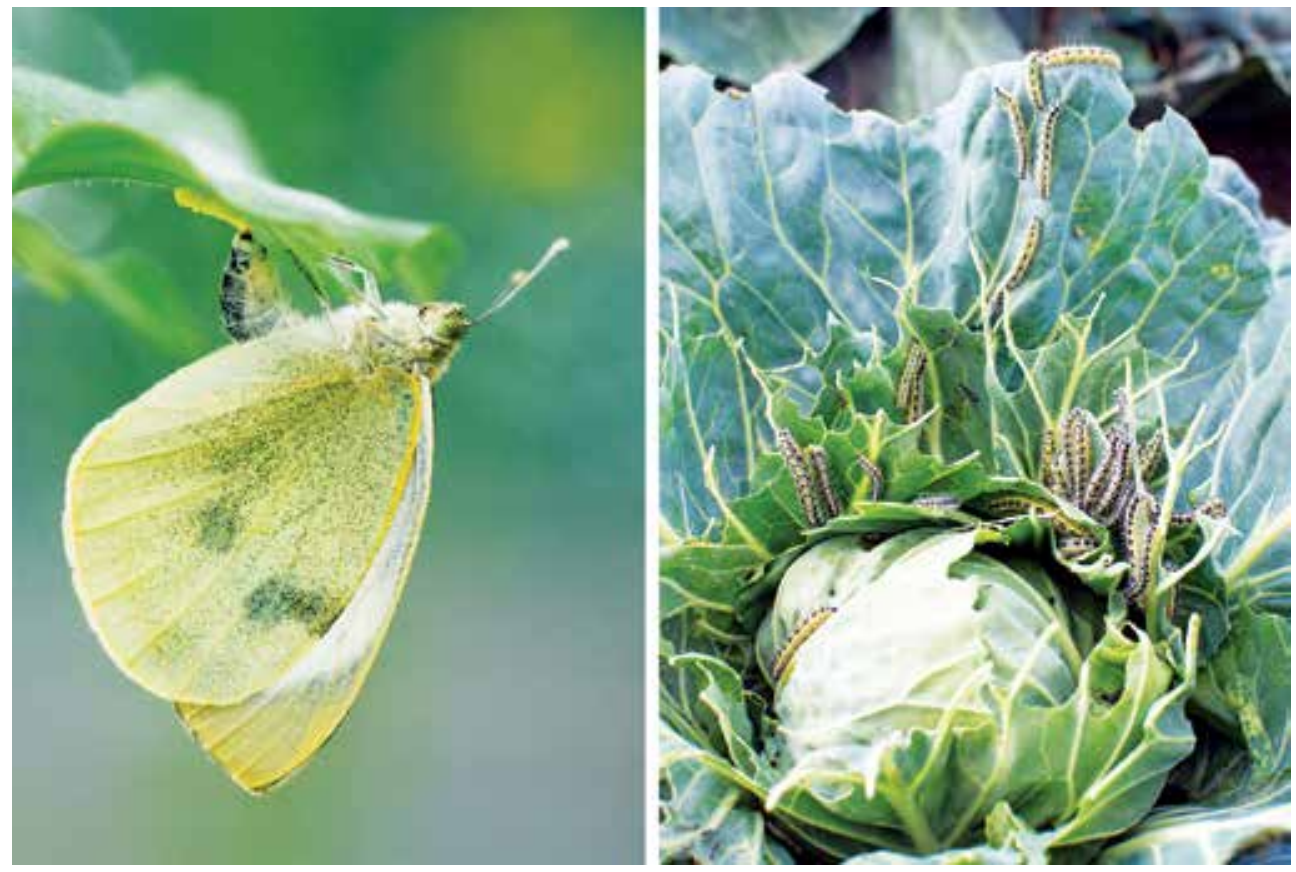

Figure 1: Pieris brassicae butterfly ovipositing (left) and late-stage caterpillars feeding from cabbage (right). Photographs by author. 
clusters of up to 150 eggs on the underside of leaves (Figure 1). Caterpillars develop through five instars. During the first instars, the caterpillars feed gregariously on a plants' leaves and flowers. In later instars, they colonize neighbouring plants as well. The species can cause excessive damage to cabbage plants in particular (Figure 1) and is considered a pest in cabbage crops. The caterpillars are at risk of being attacked by different carnivorous insects, such as the parasitoid Cotesia glomerata and by social wasps in the family Vespidae (Hymenoptera) (Lucas-Barbosa et al., 2014).

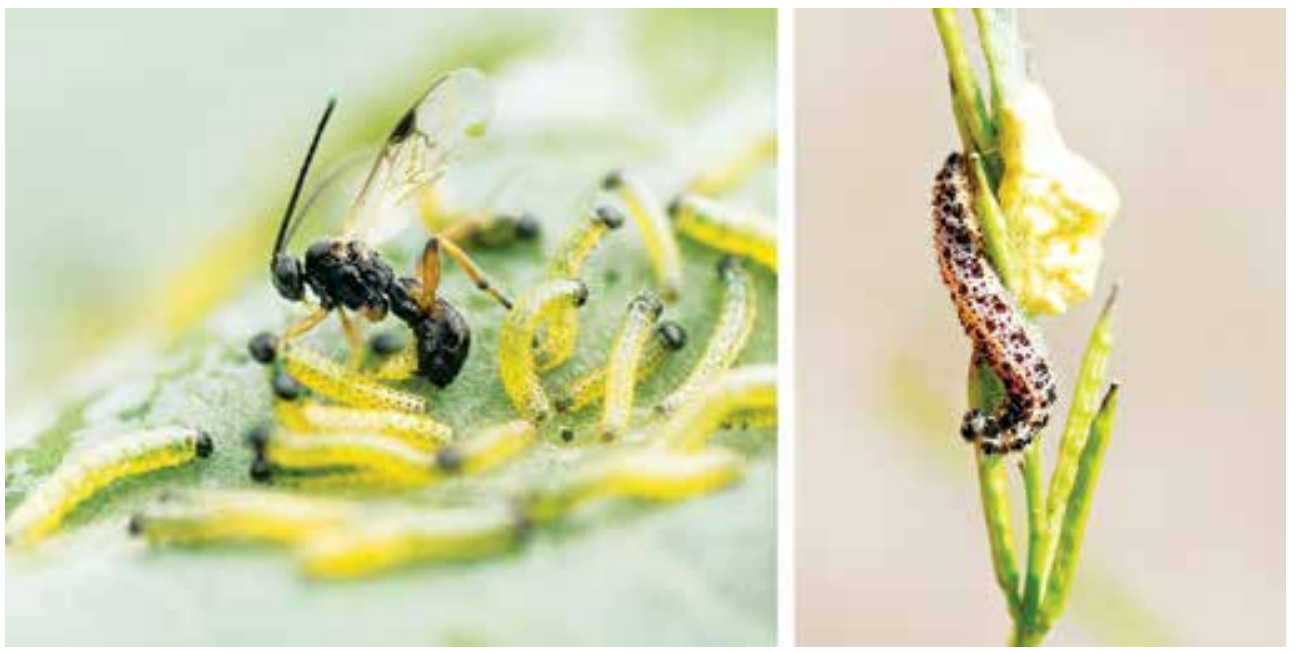

Figure 2: Cotesia glomerata attacking Pieris brassicae caterpillars (left) and a clutch of $C$. glomerata cocoons after egression from the host (right). Photographs by author.

\section{Parasitoid species}

Cotesia glomerata (Hymenoptera: Braconidae) is a gregarious larval endoparasitoid which has several host species in the lepidopteran family Pieridae, with Pieris brassicae as the preferred host (Brodeur et al., 1996). The female parasitoid lays clusters of up to 30 eggs in a single host, but is able to parasitize multiple hosts during their lifetime. The same or a different parasitoid individual may choose to lay eggs in the same host caterpillar, which is known as (self)superparasitism (Poelman et al., 2013). First or second instar caterpillars are the preferred stage for oviposition (Brodeur et al., 1996, Figure 2). As a koinobiont parasitoid, the host continues feeding and growing while the larvae are developing. When larval development is completed, larvae egress from the caterpillar and pupate outside of the host's body (Figure 2). Adult C. glomerata feed on nectar sources, which increases their longevity and flight capacity (Wäckers, 2001; Wanner et al., 2006). 


\section{Thesis outline}

In Chapter 2, I review the literature on the spatial scale at which HIPVs affect tritrophic interactions. I identified key knowledge gaps that impede our understanding of how herbivore-induced plant volatiles influence parasitoid foraging decisions as a long-distance cue. Little is known on the distance from which HIPVs can be perceived and how attributes of habitat characteristics may influence this distance.

Chapter 3 addresses the distance from which parasitoids can use HIPVs in host location behaviour and the effect of plant-trait variation in HIPV emission on this distance. In a field experiment, cabbage plants were placed at different distances from a central parasitoid release point, with a spacing of $10 \mathrm{~m}$ or 20 $\mathrm{m}$. Additionally, detailed observations on parasitoid behaviour were made in a tent, where host-plant-finding efficiency and search time could be assessed for distances up to 8 meter.

Besides being dependent on the distance to the plant, parasitoid host-seeking behaviour can also be influenced by habitat characteristics. In Chapter 4, I studied the effect of spatially heterogeneous environments on host abundance and parasitism rates. I conducted a factorial field experiment with white cabbage accessions that differed in the attractiveness of the HIPV profile for parasitoids. I modified the plot size and made changes regarding presence of a Brassica nigra border. In addition, I assessed parasitism rates of experimentally introduced Pieris brassicae caterpillars and the presence of naturally occurring Pieris spp. caterpillars for each combination of accession, plot size and border.

Structural aspects within a habitat patch are not the only factors affecting parasitoid foraging behaviour. The presence of other herbivore species which cannot serve as a host to the parasitoid could alter the plant's response to herbivory and therefore the emission of HIPV blends by the plant. In Chapter 5, I assessed parasitoid foraging efficiency and preference for plants, with variation in attractiveness, via HIPVs and in the presence of a non-host caterpillar under laboratory and field tent conditions. Additionally, I collected volatiles from plants 
of the two accessions infested with either no herbivores, only hosts, only nonhosts and a combination of hosts and non-hosts to analyse the composition of the volatile blends the parasitoids are exposed to while foraging.

In Chapter 6 I combined plant-trait variation with aspects of the surrounding landscape. I studied how plant variation and, by extension, attractiveness to parasitoids, may impact parasitism rates on these plants in 19 different landscapes. I also researched the role of landscape aspects therein, such as alternative host plant cover or land use classes. Several times during the season, patches of white cabbage were established in different landscapes. Parasitism rates pertaining to these plants have been assessed based on these findings.

In Chapter 7 I integrate the findings of the previous chapters and synthesize how variation in HIPVs affects the ability of parasitoids when it comes to finding their host in the volatile mosaic, with a particular focus on context-dependence. This final chapter makes recommendations for the application of my work and proposes future research directions.

\section{Acknowledgements}

I thank Erik Poelman, Wopke van der Werf, Felix Bianchi and Marcel Dicke for their constructive comments on an earlier version of this chapter. 


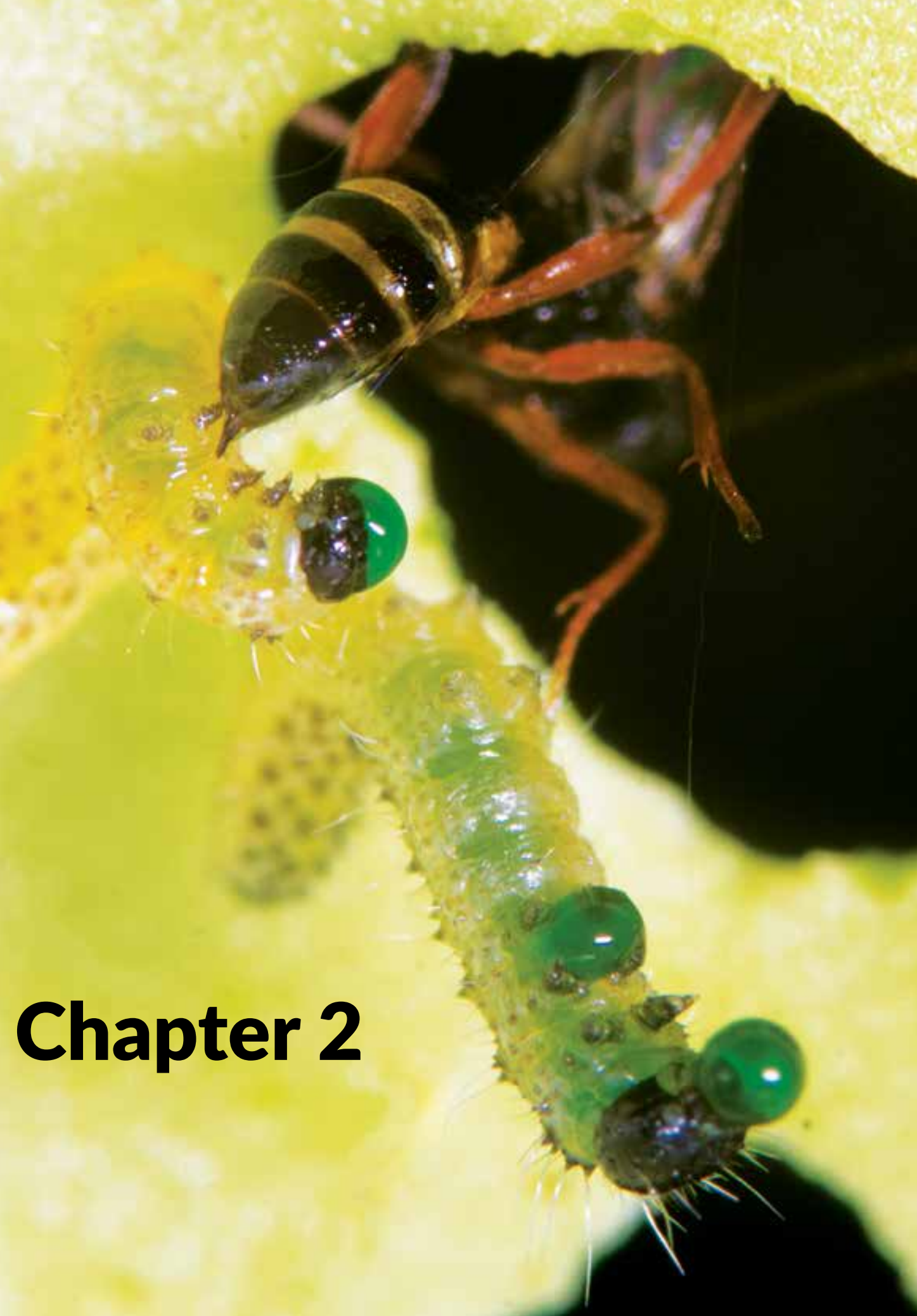




\section{Herbivore-induced plant volatiles and tritrophic interactions across spatial scales}

Yavanna Aartsma

Felix J.J.A. Bianchi

Wopke van der Werf

Erik H. Poelman

Marcel Dicke

New Phytologist (2017)

216: 1054-1063 


\begin{abstract}
Herbivore-induced plant volatiles (HIPVs) are an important cue used in herbivore location by carnivorous arthropods such as parasitoids. The effects of plant volatiles on parasitoids have been well characterized at small spatial scales, but little research has been done on their effects at larger spatial scales. The spatial matrix of volatiles ("volatile mosaic") within which parasitoids locate their hosts is dynamic and heterogeneous. It is shaped by the spatial pattern of HIPVemitting plants, the concentration, chemical composition and breakdown of the emitted HIPV blends, and by environmental factors such as wind, turbulence, and vegetation that affect transport and mixing of odour plumes. The volatile mosaic may be exploited differentially by different parasitoid species, in relation to species traits such as sensory ability to perceive volatiles and the physical ability to move towards the source. Understanding how HIPVs influence parasitoids at larger spatial scales is crucial for our understanding of tritrophic interactions and sustainable pest management in agriculture. However, there is a large gap in our knowledge on how volatiles influence the process of host location by parasitoids at the landscape scale. Future studies should bridge the gap between the chemical and behavioural ecology of tritrophic interactions and landscape ecology.
\end{abstract}




\section{Introduction}

Information plays an important role in behavioural choices of individuals, and consequently influences spatial distribution of populations on larger scales (Vet, 2001; Lof et al., 2008; Vinatier et al., 2011a). Animals have evolved many sensory systems for perceiving cues from their environment, such as vision, hearing, smell and sensing of vibration, and they use a combination of these to make foraging decisions (Roitberg \& Gillespie, 2014; Schellhorn et al., 2014). In insects, olfaction is the most important sensory system driving behaviour; it influences among others food searching, mate finding, avoidance of enemies and competition (Lima \& Dill, 1990; Schoonhoven et al., 2005). However, little is known about the mechanisms underlying the interactions between insects and their odorous environment in the context of the spatial scales at which these mechanisms need to operate under field conditions.

Herbivore-induced plant volatiles (HIPVs) constitute important cues for parasitoids and predators to find prey or hosts (Vet \& Dicke, 1992; Hare, 2011). Undamaged plants emit relatively low levels of volatiles. Upon herbivory, plants emit an induced blend of volatiles of different chemical classes (Fig. 1), produced through a variety of biosynthetic pathways. This blend is used by predators and parasitoids as a reliable and well-detectable cue to find herbivore-infested plants (Dicke \& Baldwin, 2010). While tritrophic interactions mediated by plant volatiles have been extensively studied in the laboratory and small-scale field experiments (Mumm \& Dicke, 2010), many questions remain unanswered about how these interactions unfold beyond the plot/field scale in agroecosystems (James \& Price, 2004; Simpson et al., 2011).

HIPVs are emitted from plant sources that are heterogeneously distributed at various spatial scales. Individual plants of different species may be induced to different degrees, and by different inducing herbivores, resulting in a complex spatial mosaic of volatile blends. Emitted HIPVs will be transported by wind and turbulence, resulting in mixing of multiple volatile blends, while chemical breakdown will happen at the same time. The compounding of spatially and temporally heterogeneous emission and turbulent transport results in a dynamic 
and heterogeneous three-dimensional chemical environment, which we here call the 'volatile mosaic'. Parasitoids and predators may be able to derive important information from HIPVs within this volatile mosaic, but they may be limited in their ability to detect HIPVs at larger spatial scales due to chemical breakdown of chemical constituents, and mixing of odours from different sources. Furthermore, they may be limited in their ability to initiate directed movement towards these potential sources of hosts/prey, for example if wind speed exceeds the speed of movement. Therefore, the interactions between carnivorous insects and the volatile mosaic are likely to be scale-dependent, such that different processes may be relevant at different spatial scales.

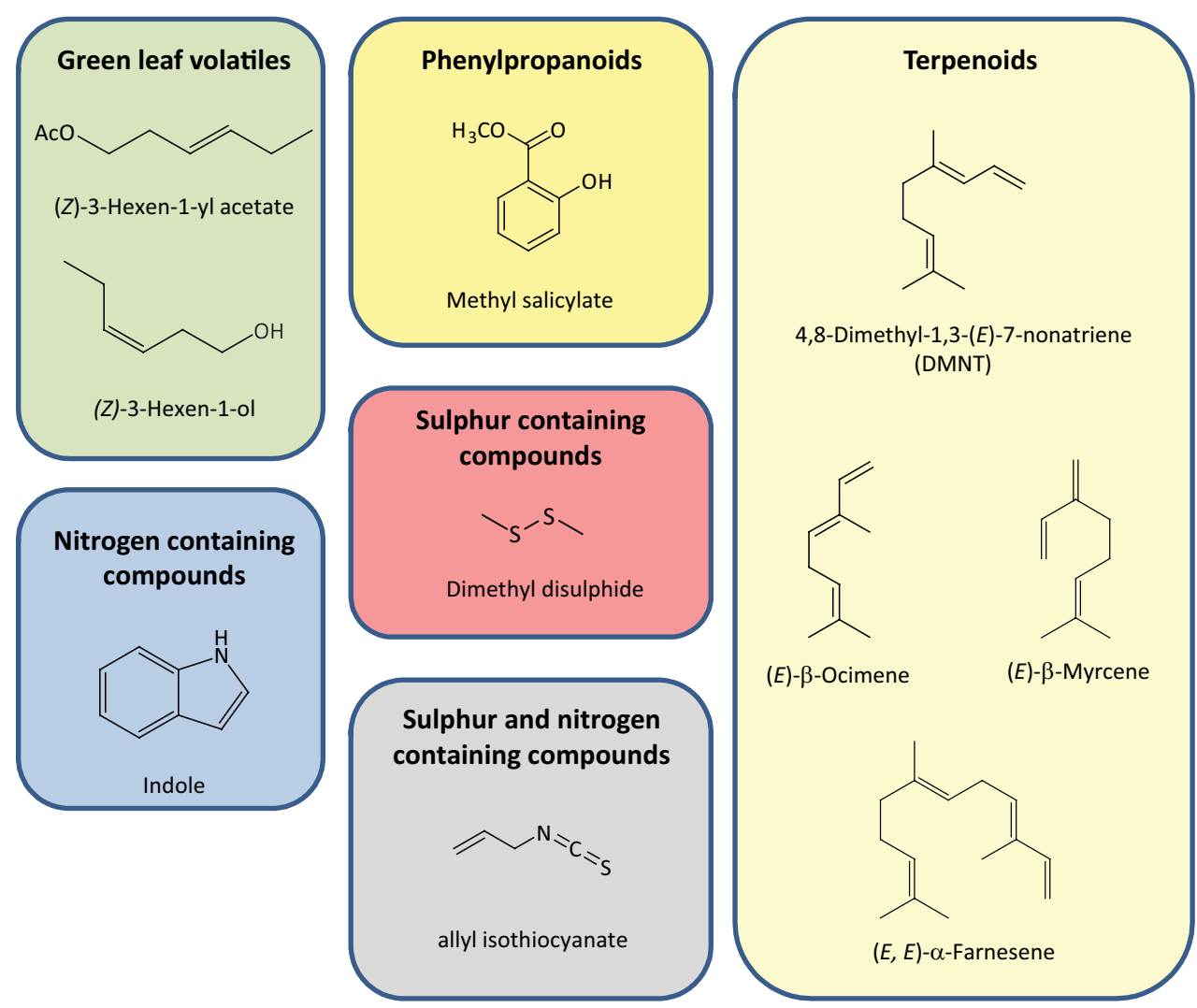

Figure 1: HIPVs consist of chemicals from different chemical classes. Examples for several different classes of compounds that can be found in HIPV blends. 
In this review, we argue that volatile mosaics, by influencing parasitoid choices and, consequently, parasitoid movement, may be a helpful addition to the current suite of landscape ecological concepts, such as structural complexity, fragmentation and connectivity. Volatile mosaics may allow for a more mechanistic understanding of the movement and distribution patterns of organisms that are especially driven by olfactory cues. Although many carnivorous arthropods use volatile information, we limit our review to interactions between plants, herbivores and primary parasitoids, which lay their eggs in herbivorous hosts. The use of information from their surroundings by primary parasitoids has been extensively studied (van Alphen et al., 2003), and their fitness is closely linked to their ability to use volatile information to find hosts (Thiel \& Hoffmeister, 2009). We first address the physical characteristics of volatile mosaics and the factors that shape them. Second, we provide information on how parasitoids perceive their environment and how their physical and behavioural traits might influence the extent to which HIPVs are used in a landscape context. Third, we discuss three different spatial scales at which volatile mosaics may influence parasitoid movement and distribution, namely the plant scale, patch scale and landscape scale. Finally, we discuss future research directions, open questions and potential applications of HIPVs for strengthening biological control in agricultural systems.

\section{Formation of the volatile mosaic and insect behavioural traits}

Volatiles emitted by plants form plumes that consist of odour filaments (Murlis et al., 1992; Beyaert \& Hilker, 2014). These plumes provide information to parasitoids that search for their herbivorous hosts (Dicke \& Baldwin, 2010). How this information can be used by parasitoids depends on the sender (the plant), the processes affecting the shape and spatial extent of odour plumes in the environment, and the ability of the receiver (the parasitoid) to perceive the cue (Fig. 2). In this section we discuss these three aspects, of which the first two form the volatile mosaic and the third determines how the volatile mosaic is perceived by the parasitoid. 
The production and release of a blend of volatiles starts at the level of the plant. Plant responses to herbivory have been extensively investigated, highlighting that the blend composition may vary with herbivore species, density and herbivore instar (D’Alessandro \& Turlings, 2006; Mumm \& Dicke, 2010; Rowen \& Kaplan, 2016), abiotic conditions (Loreto et al., 2014) and plant species, cultivar or even genotype (Degen et al., 2004; Poelman et al., 2009b; Gols et al., 2011).

After the odour blend leaves the plant as part of a plume, air currents determine the direction and speed at which the plume travels (Riffell et al., 2008). Volatile compounds in the atmosphere can gradually degrade, for example by interactions with reactive chemicals such as ozone (Blande et al., 2014). The degradation of compounds can alter the chemical composition of the blend by changing the ratio of compounds within the blend, and/or generating new breakdown products (Šimpraga et al., 2016). With increasing distance from the source, a plume becomes more dispersed and probably more difficult to be tracked by

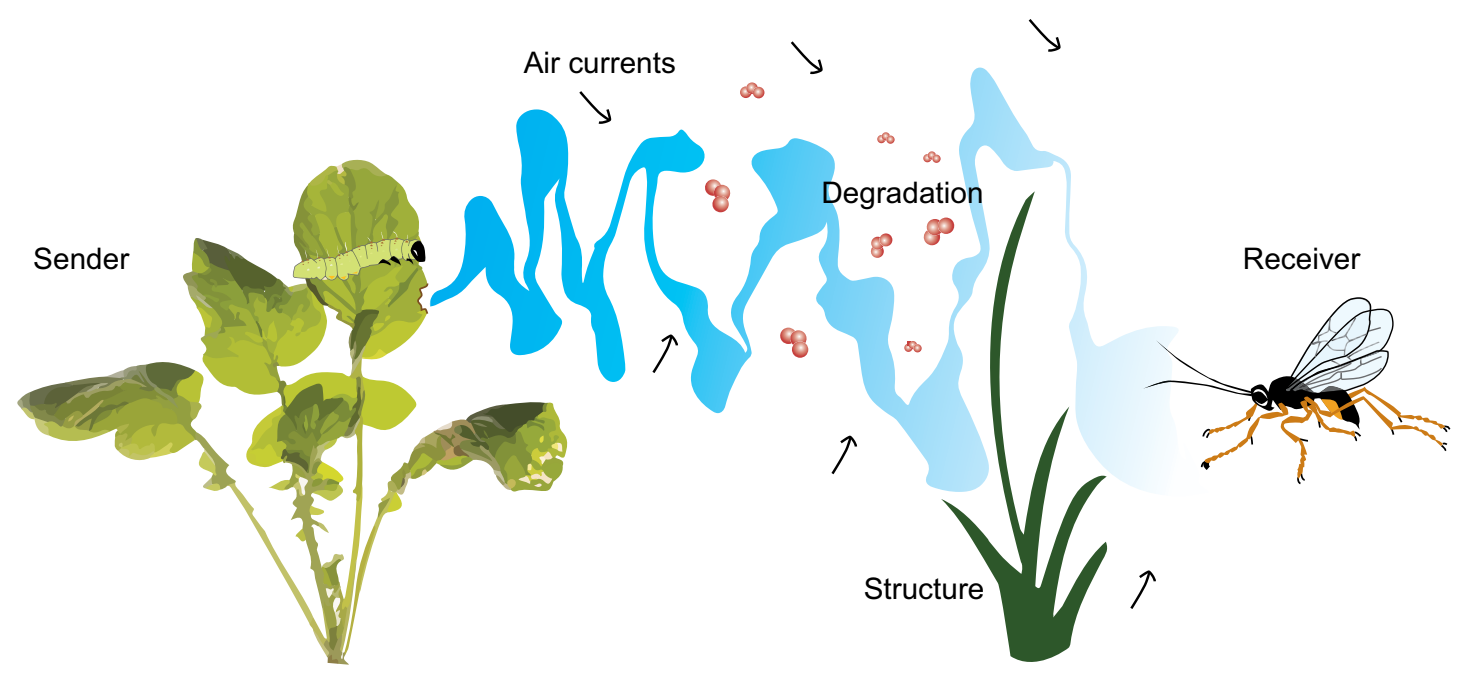

Figure 2: The sender (plant) emits herbivore-induced volatiles, which disperse as plumes in the environment as a result of air movement. Physical barriers such as vegetation further modulate the movement pattern of the volatile plume. With increasing distance from the source, the plume becomes more fragmented as a result of degradation, by reactions with other compounds in the atmosphere, and dilution as airflow spreads the plume. Depending on the distance from the source and traits of the receiver (insect parasitoid), the receiver may be able to follow the odour plume to the source. 
parasitoids. These processes ultimately determine the shape, concentration and spatial extent of the odour plume, as well as the composition of the odour filaments, which can alter the information available to insects. In a landscape setting, insects are confronted with assemblages of plants producing odour plumes that differ in blend, strength and size. Limited knowledge is available on the responses of parasitoids to mixed odour plumes (Dicke et al., 2003), but mixing of plumes may give rise to complex interactions such as plume masking or plume amplification. For instance, when moths are exposed to a mixture of pheromone and plant volatiles the capacity of pheromone detection is hampered, probably because of a masking effect of plant volatiles (Deisig et al., 2014).

Volatile mosaics consist of assemblages of odour plumes that are scattered across space and can be influenced by the vegetation structure of the landscape. For instance, odour plumes in open fields and forests have different shapes and sizes, possibly due to the differences in wind speed and turbulence in these contrasting habitats (Murlis et al., 2000). Increasing plant diversity is expected to increase the structural complexity of vegetation, but can also increase complexity of the volatile mosaic by mixing of odour plumes (Randlkofer et al., 2010b). The complex interaction between the spatial arrangement of plant communities in the wider landscape context and environmental factors leads to a bewildering array of emerging patterns, which are likely to change rapidly over time. Yet, parasitoids have to deal with this complexity to obtain olfactory information about the location of their hosts.

The perception of the volatile mosaic by parasitoids is determined by their ability to detect and interpret volatiles. Sensory perception of volatiles by insects relies on olfactory sensilla, primarily located on the antennae. These sensilla are innervated by olfactory receptor neurons, and a wide variety of receptor neuron types can be found among insect taxa (Martin et al., 2011; Reinecke \& Hilker, 2014). Parasitoid species may differ in their ability to detect volatile compounds, which impacts their ability to discriminate between volatile blends, and the distance from which they can track volatile-emitting plants (Gouinguené et al., 2005). The minimum volatile concentration eliciting a behavioural response may vary between parasitoid species. We expect a positive correlation between 
sensitivity of a parasitoid species to a particular volatile blend and the distance from which the blend can be detected from the source (as chemical breakdown and dilution due to turbulence have reduced the volatile concentration and altered the composition). Detection of odour plumes in a three-dimensional environment is complicated because of turbulence and chemical degradation of the plumes over larger distances. Complex navigational strategies are employed by insects to locate the source of the odour plume, for instance flying in a zigzagging fashion upwind towards the source of the volatiles (Kaiser et al., 1994; Kerguelen \& Cardé, 1997; Cardé \& Willis, 2008). When following odour plumes, insects may change their navigational strategy at certain distances from the odour source (Willis et al., 1991; Bau \& Cardé, 2015).

Detection ability is not in itself sufficient to locate a host. Parasitoids should also have the physical ability for directed movement to search and locate the host if they detect HIPVs. We expect flight capacity to influence the scale over which a volatile mosaic is explored and the spatial grain of searching. At low flight capacity, a parasitoid may intensively explore small patches of plants, and may depend on passive dispersal for finding patches further away, while a parasitoid with good capacity for directed flight may visit a sequence of interconnected resource patches by flying upwind in the direction of an odour source. There are several factors influencing the movement capacity of insects. In general, there is a positive correlation between the size of a parasitoid and their movement capacity (Roland \& Taylor, 1997). However, even individuals within the same species may exhibit different modes of movement, resulting in displacement across distances ranging from metres to kilometres (Kristensen et al., 2013a). Host-specific parasitoids may be more mobile and sensitive to specific volatiles than parasitoids with a wider host range (van Nouhuys \& Ehrnsten, 2004). 


\section{The spatial scales of parasitoid interactions with plant volatiles}

A parasitoid female emerging from her cocoon has only limited time to explore the environment and obtain information on patch quality. Perceptual range, resulting from perception sensitivity and odour dispersal, will influence host finding when hosts are heterogeneously distributed. However, not much is known about odour perceptual range of parasitoids in field situations, or whether this range differs between species. Some studies with artificial volatile sources and moths show antennal responses to odour sources in the field up to $60 \mathrm{~m}$ from the odour sources, depending on the number of odour sources (Andersson et al., 2013). The distance over which odours are perceived also depends on the landscape, which determines how far odours travel. For example, tsetse flies respond to host odours from a much larger distance $(60 \mathrm{~m})$ in woodlands than in open fields $(20$ $\mathrm{m})$, suggesting that odour plumes stay intact longer in these vegetation structures (Voskamp et al., 1998). Weather also affects perceptual range by influencing odour plume movement. While plants can convey information on attack by herbivores (Turlings et al., 1990; Vet \& Dicke, 1992), the detection and interpretation of these cues by parasitoids may differ depending on the distance of the parasitoid to the HIPV source, although empirical evidence for this is lacking (Puente et al., 2008). Depending on the spatial scale, different factors may be of overriding importance. Here, we will review the most important factors affecting the response of parasitoids to HIPVs at the plant, patch and landscape scale.

\section{Plant scale}

HIPV release at the plant scale is the basis of the formation of the volatile mosaic, which can be modulated by a wide range of factors, including plant species, plant genotype, plant age, herbivore species, attack severity, abiotic factors, or combinations of these (Fig. 3A). The interplay of these biotic and abiotic factors results in specific outcomes of tritrophic interactions at the plant scale in which volatiles may convey reliable information to parasitoids about the infestation of plants by herbivores, while in other cases the volatile cues are less specific (de Rijk et al., 2013). 
(A) Plant scale

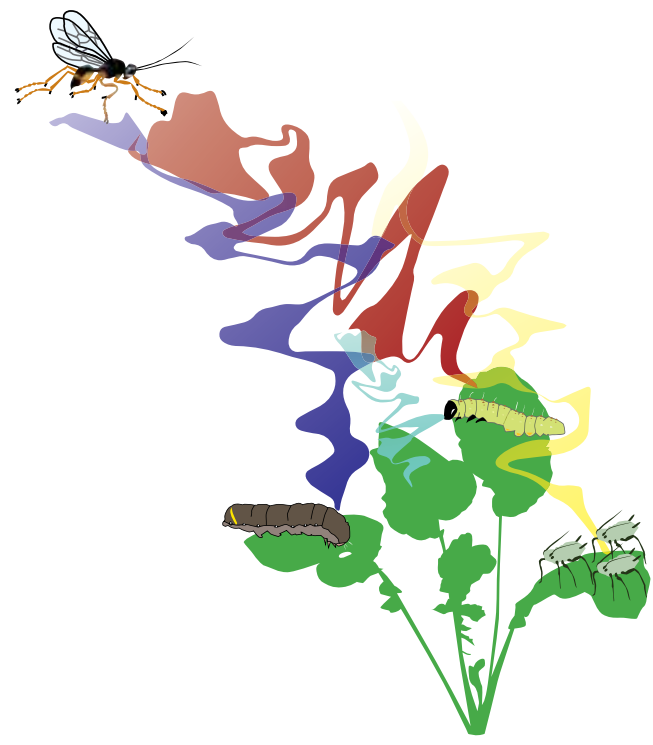

(B) Patch scale

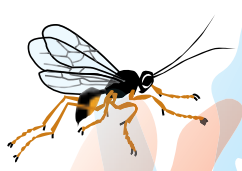

(C) Landscape scale

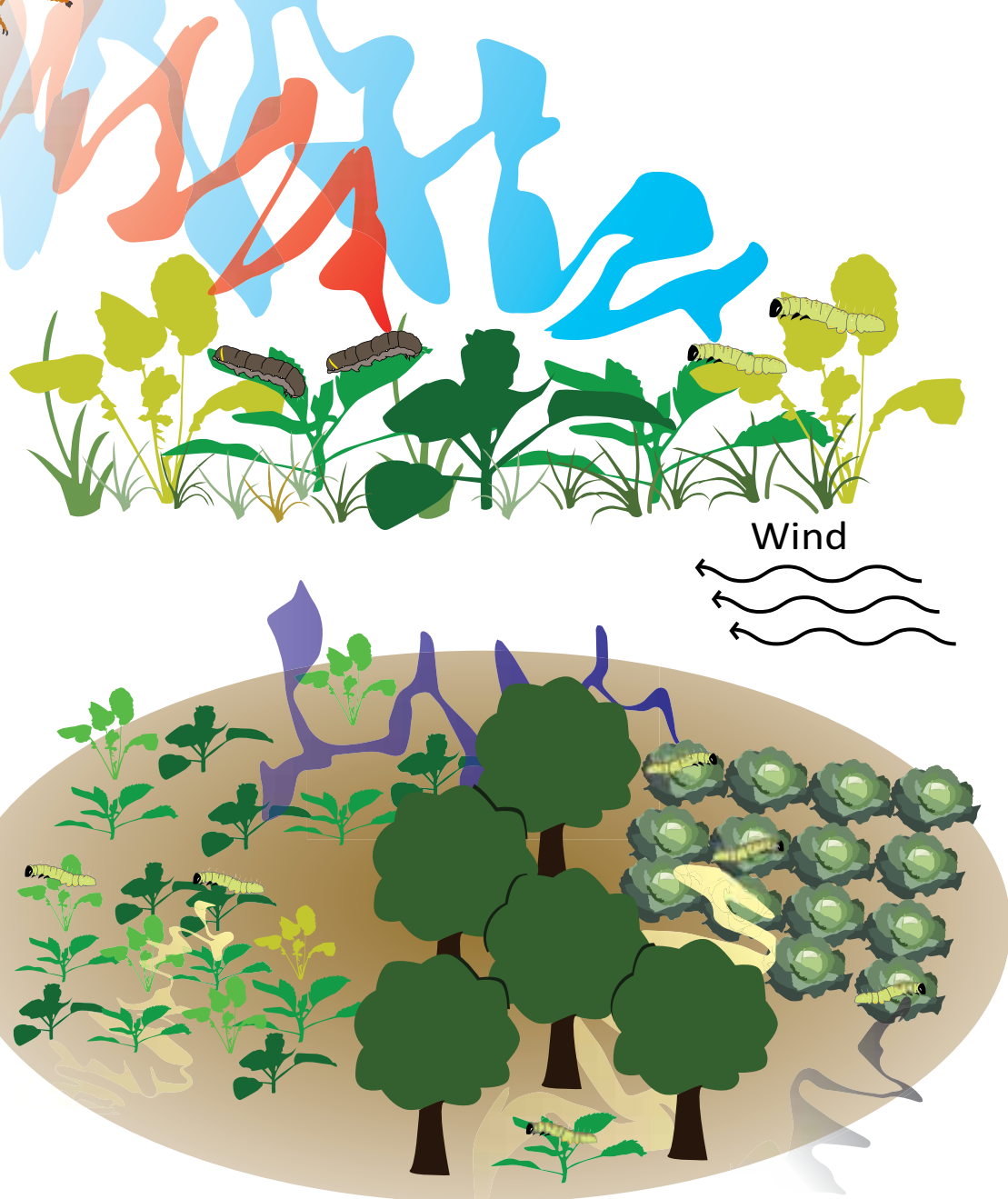


Herbivore-damaged plants emit a blend that is qualitatively and/or quantitatively different from the blend emitted when the plant is not damaged or mechanically damaged (Turlings et al., 1990; Ponzio et al., 2014). As a consequence, plants damaged by host herbivores attract more parasitoids than uninfested or mechanically damaged plants (Turlings et al., 1990; Geervliet et al., 1994; Potting et al., 1995). HIPV emission is positively related to the severity of herbivore damage and herbivore load (but see Shiojiri et al. 2010) and, consequently, more heavily infested plants are more attractive to parasitoids (Girling et al., 2011). Phloem-feeding herbivores generally induce lower amounts of volatiles than chewing herbivores (Rowen \& Kaplan, 2016), possibly because of the limited tissue damage caused by phloem feeders. Besides affecting initial parasitoid attraction to a plant, HIPVs can further stimulate searching behaviour when the parasitoid has arrived on the plant (Uefune et al., 2012).

Plant traits can modulate HIPV release and plant volatile emission fluctuates throughout the day (Loughrin et al., 1994; Arimura et al., 2008), highlighting the dynamic nature of volatile mosaics. Plant species emit specific volatile blends upon attack by the same herbivore species (van den Boom et al., 2004). Genotypes or varieties of the same plant species may differ in the intensity of volatile emission (Degen et al., 2004; Poelman et al., 2009b; Gols et al., 2011), which may result in contrasting parasitism rates under field conditions (Poelman et al., 2009b). Additional infestation by non-host herbivores may alter HIPV emission and, consequently, parasitoid attraction (de Rijk et al., 2013; Chabaane et al., 2015; Ponzio et al., 2016). Different non-host-herbivore species may vary

Figure 3 (left): Herbivore-induced plant volatiles (HIPVs) on multiple spatial scales. A) A plant can respond to herbivory with the production of HIPVs. The composition of these volatile blends is affected by many on-plant factors, such as herbivore identity, herbivore feeding guild, herbivore community, and plant species or traits. Parasitoid responses may vary with variation in HIPV blends. B) In nature, the plant is part of a larger community of plants and their associated herbivores. Therefore, parasitoids search for their hosts in patches where a large variety of odour plumes shapes the information on presence of the host, representing a dynamic volatile mosaic. C) At the landscape scale, different habitats can present different volatile mosaics and distance between these habitats becomes important. Landscape structure as determined by openness of the vegetation and plant diversity, as well as weather conditions such as wind direction affect how far odour plumes travel. The ability of the parasitoid to perceive HIPVs emanating from patches further away and to move across these habitats influences movement patterns and resulting population distribution. 
in the degree to which their attack alters HIPV blends and influences parasitoid searching behaviour (Desurmont et al., 2014; de Rijk et al., 2016a). Hence, the contribution of a single plant to the volatile mosaic depends on the attacking insects, both hosts and non-hosts.

\section{Patch scale}

At the patch scale, the complexity of plant communities contributes to the complexity of the volatile mosaic (Fig. 3B). Abiotic conditions, such as wind, influence the distribution of volatiles (Loreto et al., 2014; Li et al., 2016) and vegetation structure may further modulate the dispersal of volatile blends within the landscape. The variation in HIPV emission between and within plant species is likely to shape volatile blends in complex ways (Degen et al., 2004; Poelman et al., 2009b; Gols et al., 2011), which then changes the volatile mosaic depending on the neighbouring plants and the herbivore community that is present on these plants. The effect of plant diversity and habitat complexity on parasitoid behaviour has been extensively studied (Wäschke et al., 2014; Kruidhof et al., 2015). For example, Brassica nigra plants in unmown grassland attracted fewer parasitoids than those in mown grassland or bare soil (Bezemer et al., 2010). Parasitoids found host-infested plants faster in a Brussels sprouts monoculture compared to a Brussels sprout - barley intercrop (Bukovinszky et al., 2007). It is unclear whether these differences are caused by volatile masking, mixing of volatiles, or obstruction of visual or olfactory cues by the vegetation. Several effects of background odours on parasitoid behaviour in a patch have been described. If a parasitoid is unable to perceive a certain compound or blend, it is likely that this compound does not alter its behaviour in a patch (Schröder \& Hilker, 2008). A background odour might attenuate a behavioural response if it masks the target odour, or enhance the response if it complements the signal of the HIPV emitting plant (Schröder \& Hilker, 2008).

The induction of HIPVs in neighbouring plants infested with non-host herbivores can stimulate the searching efficiency of parasitoids by creating a contrasting HIPV blend that can help the parasitoid to identify the HIPV blend 
of host-infested plants (Soler et al., 2007; de Rijk et al., 2013). Yet, this effect may depend on the herbivore species inducing the neighbouring plant. For instance, discriminating hosts from non-hosts on the basis of HIPVs is more difficult for parasitoids when the herbivores are from the same feeding guild than from a different feeding guild (Geervliet et al., 1996; de Rijk et al., 2013). The attractiveness of neighbouring plants can also influence the searching behaviour of parasitoids on host-infested plants. When an attractive host plant was surrounded by less attractive, but still attractive non-host plants, the searching efficiency on the host plant decreased, suggesting that the perception of the patch quality exceeds the scale of a single plant (Perfecto \& Vet, 2003). HIPVs can attract parasitoids to host patches, and may even be used to assess patch quality. Aphid parasitoids that were previously exposed to a plant with a high aphid density spent less time on plants with few or no aphids than parasitoids that were previously exposed to a plant with a low aphid density. This was independent of actual presence of aphids during the exposure period, suggesting that plant volatiles were used by the parasitoids to assess patch quality (Tentelier \& Fauvergue, 2007).

Theoretical studies with plume models suggest that odour sources of which the plumes can be perceived at larger distances attract more insects than those than those that can be perceived at only a short distance from the source (Manoukis et al., 2014). Long-distance spread of odour plumes facilitated host location if hosts were sparsely distributed, while short- or long-distance spread of plumes equally facilitated host location if hosts were dense (Puente et al., 2008). The release of the synthetic HIPV component phenylethyl alcohol influenced the community composition and abundance of a range of arthropod taxa (both second and third trophic level) up to at least $8 \mathrm{~m}$ away, both positively (by attraction) and negatively (by repellence) in soybean fields (Braasch \& Kaplan, 2012). In contrast, Mallinger et al. (2011) found that such effects were more localized and ceased at $1.5 \mathrm{~m}$ from the source. These spatial characteristics of the response to odour plumes are likely to be both plant- and insect-species specific. 


\section{Landscape scale}

Landscapes are composed of a mosaic of vegetation patches, each consisting of plants that may produce HIPV plumes. However, only few studies have examined how HIPVs influence the movement of parasitoids at a landscape scale and how HIPVs from different patches influence the distribution of parasitoids across a landscape (James \& Price, 2004; Simpson et al., 2011). The study of HIPVs in a landscape context presents a challenge because of the difficulty of tracking parasitoid movement at large spatial scales, and because HIPV plumes are not visible and, therefore, hard to assess in the field. Indeed, most landscapescale studies infer parasitoid movement via indirect methods, such as analysis of meta-population structure, rather than by assessing the movement paths of individual parasitoids (Schellhorn et al., 2014). Nevertheless, considering HIPV plumes may reveal important insights in the movement and distribution patterns of parasitoids at the landscape scale.

Landscape-ecological studies have shown that forest edges, proportion semi-natural area or landscape simplification can have profound impacts on the distribution of parasitoids and their impact on herbivore populations (Pollard \& Holland, 2006; Bianchi et al., 2008; Woltz et al., 2012; Rusch et al., 2016), and that parasitoids respond to the landscape context at spatial scales ranging from several hundred metres to kilometres (Thies et al., 2003; Bianchi et al., 2008). While it has been shown that habitat types and vegetation structures may foster or impede parasitoid movement (Cronin, 2003a; Cronin, 2003b), it is not clear to what extent HIPVs play a role in this. The volatile mosaic can be considered as an additional information layer on top of the structural vegetation pattern. Depending on the spatial arrangement of vegetation patches emitting HIPVs and meteorological conditions that determine the shape and direction of odour plumes, the volatile mosaic may facilitate parasitoid movement (e.g. when nearby odour plumes function as stepping stones) or interfere with it (e.g. when attractive odour plumes are masked by less attractive plumes), in similar ways as found for vegetation structures (Tscharntke \& Brandl, 2004) (Fig. 3C). While the spatial vegetation template varies at a seasonal time scale, the volatile mosaic is much more dynamic and may change within seconds to minutes depending on wind conditions, turbulence and vegetation structure. 
Understanding the interactions between parasitoids, herbivores and plants in a volatile-mosaic context requires the integration of various factors that have been addressed in this review. In a landscape, parasitoids need to find all ecological requisites, including food resources, hosts and mates, and they need to allocate time to finding these resources at appropriate times of their lives (Lewis et al., 1998; Landis et al., 2000). The perception of the volatile mosaic may be very different depending on the scale and mode of movement of the parasitoid. For instance, volatile mosaics may be perceived as fragmented by parasitoids with a limited mobility, while less so by parasitoids with a large dispersal capacity (van Nouhuys, 2005). Further work is needed to unravel the factors and mechanisms that underlie the parasitoid movement and host searching at the landscape scale.

\section{Future perspectives}

The previous sections show that knowledge of parasitoid responses to HIPVs within the volatile mosaic decreases with increasing spatial scale. At present, there are no accurate data on the spatial extent of HIPV plumes. Beyond a critical distance, it can be expected that HIPV plumes are simply too diluted or dispersed by turbulence or chemically degraded, so that no reliable information can be derived from them by parasitoids. We hypothesise that HIPV plumes may provide reliable cues for parasitoids up till a distance of tens of metres, in line with studies on flies and moths (Voskamp et al., 1998; Andersson et al., 2013) and parasitoids (Y. Aartsma, pers. obs.). At further distances other cues will have overriding importance. It has been proposed that herbivores, pollinators and parasitoids use general 'habitat cues' to find locations that potentially contain resources, and then switch to more specific cues within this habitat (Vinson, 1976; Webster \& Cardé, 2016). Indeed, in no-choice situations or choice situations against non-host plant species, parasitoids often also respond to volatiles from undamaged plants, indicating that in the absence of host-specific HIPVs, more general cues are used (Gohole et al., 2005; Moraes et al., 2008). Hierarchical plume switching is a possible mechanism by which flying insects following a long-range habitat cue might switch to following more reliable short-range cues (Beyaert \& Hilker, 2014). A better understanding of the functioning of HIPVs in realistic field conditions requires 
characterization of the distance over which odour plumes can attract parasitoids in different plant-herbivore combinations.

Assessing the response of parasitoids to volatile mosaics is methodologically challenging, especially at the field scale and beyond. Here, we propose three potential approaches that integrate approaches from chemical ecology and landscape ecology that may foster progress in this field. First, the mechanistic basis of the volatile mosaic can be studied by collecting HIPV blends under field conditions in habitats with different structural complexity. By presenting field-collected or synthetic HIPV blends to parasitoids and recording their behaviour, predictions for parasitoid behaviour and distribution in the field can be made. Parasitoids can be released at different distances from a source and recaptured near the source to determine the distance at which parasitoids respond in the field (Papaj \& Vet, 1990). Furthermore, electro-antennographic (EAG) measurements in the field can be used to study in more detail under which conditions HIPV blends are still distinguishable against a background (Milli et al., 1997; Andersson et al., 2013; Misztal, 2016). Second, the volatile mosaic may be studied at a landscape scale by assessing the spatial distribution of parasitoids in landscapes with different numbers and spatial arrangements of HIPV sources. In addition, to assess the effect of an HIPV source in specific volatile-mosaic contexts, the response of parasitoids could be studied by introducing standardized HIPV sources in different habitat patches. The effect of the strength of HIPV cues could further be assessed in detail by using plant phenotypes that clearly differ in HIPV emissions (Poelman et al., 2009b). We hypothesize that variation in relative volatile emission rate and associated variation in attraction of parasitoids and predators among plant species/genotypes are strongly dependent on the volatile mosaic in the surrounding landscape. Third, by studying different parasitoid species with well-known functional traits, such as threshold HIPV concentrations to initiate host-searching behaviour, important new insights may be acquired about how parasitoid distribution patterns in realistic landscape settings are shaped by the interaction between species traits and the volatile mosaic. We hypothesize that parasitoid species traits such as size and dispersal capacity influence the spatial scale and landscape context at which they respond to HIPVs. Finally, simulation models may be used to integrate and 
extend information about parasitoid responses to volatile mosaics. For instance, simulations suggest that wind direction and HIPV concentration are important factors determining the spatial distribution of HIPVs (Kuroyanagi et al., 2012). Such modelling studies may generate hypotheses that can be experimentally tested in field experiments (Manoukis et al., 2014).

Interactions between parasitoids and the volatile mosaic should be studied at relevant spatial scales. These relevant spatial scales may be species-specific because parasitoids differ in dispersal capacity and search behaviour, and they may also depend on vegetation structure and meteorological conditions. Parasitoids with a low dispersal capacity may lack the ability for directed movement to distant targets and may, therefore, be less responsive to cues from longer distances, while larger parasitoids with a good capacity for directed search may be more sensitive to long-range cues. However, for many parasitoids knowledge about functional spatial scales is limited.

Finally, it is important to recognise that volatile cues are not the only information available for parasitoids to find their hosts, and that host searching is only a part of the daily activities. Visual and vibrational cues are also used in host finding (Fischer et al., 2001), although these are considered short-range cues (Völkl, 2000). Moreover, vegetation structure can influence parasitoid movement patterns, not only through the volatile mosaic (Randlkofer et al., 2010b), but also by visual obstruction, physical increase of the searching area (Gols et al., 2005; Randlkofer et al., 2010a), or effects on parasitoid flight capacity. In field situations, insects are likely to use multiple modes of information acquisition (Kulahci et al., 2008). Our understanding of the interactions between parasitoids and volatile mosaics can benefit from a better integration of chemical, behavioural and landscape ecological approaches. 


\section{Applications}

In natural systems, parasitoids and predators keep populations of herbivorous insects at low levels, and they can have similar impacts in agriculture (Ramsden et al., 2016). Attracting natural enemies to crop fields therefore would be beneficial for farmers. There are indications that as a consequence of crop domestication and reliance on insecticides, many crops have reduced defences against herbivore attack and reduced attractiveness to natural enemies compared to their wild relatives (Chen et al., 2015). However, a recent meta-analysis suggests that emission rates of HIPVs, especially green leaf volatiles and sesquiterpenes, are actually higher in crops than in wild plant species (Rowen \& Kaplan, 2016). The complexity of volatile blends from domesticated crops is reduced as compared to wild species, which may mean that compounds which are important in attracting natural enemies are limiting (Rowen \& Kaplan, 2016). Modern plant breeding mainly focuses on direct mechanisms of resistance, and little attention is paid to the development of improved indirect defence mechanisms, for example through natural enemy attraction by plant volatiles (Áhman et al., 2010).

Another challenge for the application of HIPVs in pest management strategies is that the reported effectiveness of HIPVs is mixed. Success stories include the 'push-pull' system developed for maize production (Khan et al., 1997), and the effects of white cabbage varieties that are more attractive to parasitoids in the laboratory in combination with higher parasitism rate in the field (Poelman et al., 2009b). However, other studies show that parasitoid searching activity and host finding success is increased in laboratory studies, but that these changes do not result in reduced pest populations in the field (Halitschke et al., 2008; von Mérey et al., 2012; Bruce et al., 2015). This illustrates the importance of field studies in addition to detailed behavioural studies in the laboratory.

For biological control, it is important that crops can attract sufficient natural enemies for effective top-down control of herbivore populations. There have been mixed results with engineering constitutive release of plant volatiles or alarm pheromones (Bruce et al., 2015) and simulations show that HIPVs as cues do not increase parasitism rates when plants emit them in the absence of hosts 
of parasitoids (Kaplan \& Lewis, 2015). Enhancing induced crop attractiveness (by triggering a higher HIPV emission upon herbivory) might be a more useful approach to increase attraction of natural enemies within as well as into the crop (Kappers et al., 2011). Also, monoculture cropping systems are often very simplified and exposed to frequent disturbances and, therefore, rely on recruitment of natural enemies from the surrounding habitat (Wissinger, 1997). In addition, natural enemies may rely on floral resources that can only be found outside the field (but see Vollhardt et al., 2008).

Crops with enhanced HIPV emission levels may reduce natural enemy populations in neighbouring crops (Braasch \& Kaplan, 2012). Indeed, parasitoid redistribution on a local scale $(8 \mathrm{~m})$ was observed after volatile lures were employed, resulting in increased braconid parasitoid densities near the lure, but lower densities in plots further away from the lure (Braasch \& Kaplan, 2012). Other arthropod taxa, however, did not show such natural enemy depletion responses, and it is unclear what the consequences will be at larger spatial scales. This suggests that the implementation of strategies to enhance natural enemy recruitment by crops with enhanced HIPV emission must go hand in hand with habitat management to conserve and increase natural enemy populations near crops (Landis et al., 2000; Tscharntke et al., 2005a).

\section{Conclusions}

In the last few decades we have learned a lot about HIPVs in terms of (bio) chemistry, plant physiology, and behavioural ecology of insect responses to HIPVs (Turlings et al., 1990; Mumm \& Dicke, 2010). The focus of this research was especially on interactions between individual plants and a single herbivore and the response of individual parasitoids at the plant level. In more recent years, research has addressed the effects of HIPVs within a community context, again particularly at the plant level (Desurmont et al., 2014; Stam et al., 2014). Despite exciting advances in research on responses of plants to herbivory, many important questions remain unanswered about the consequences of HIPV emissions for parasitoid foraging behaviour and host-parasitoid population dynamics in field 
or landscape settings. These questions, relating to the spatial scale at which HIPVs operate, are crucial for our understanding of tritrophic interactions and possible applications of volatiles in agricultural pest management (Gish et al., 2015). Research on HIPVs will need to consider effects at larger spatial scales if it is to assess the effects on populations in a spatial context and contribute to durable pest management in an agricultural context. Current mechanistic understanding of the effect of plant volatiles on insect movement may be used to formulate empirically testable hypotheses on the role of HIPVs in ecological processes at the larger spatial scales that are important for landscape ecology and agricultural pest management.

\section{Acknowledgements}

We thank Dani Lucas-Barbosa for help with preparing Fig. 1. 
HIPV s and tritrophic interactions across spatial scales 


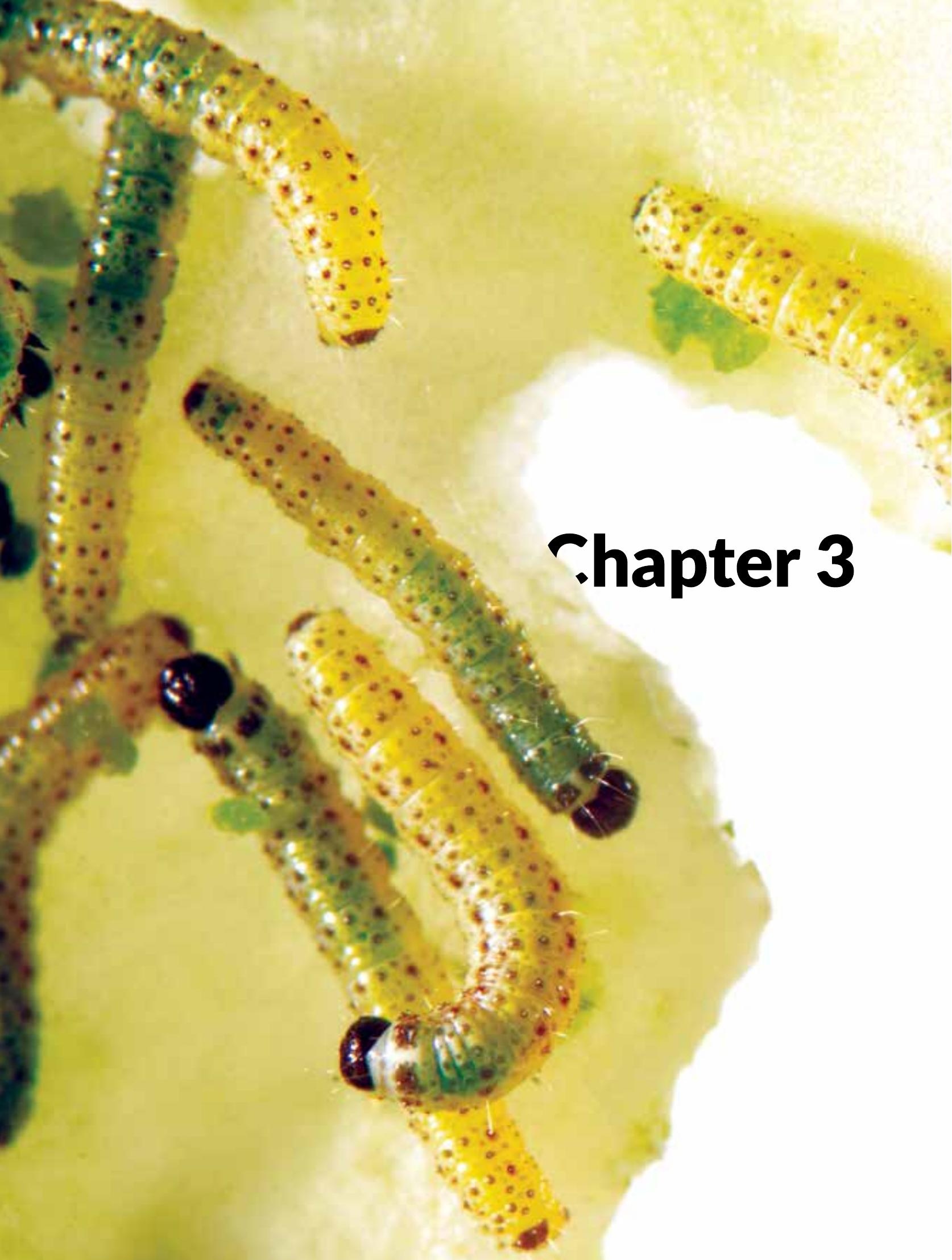




\section{Intraspecific variation in herbivore- induced plant volatiles influences the spatial range of plant-parasitoid interactions}

Yavanna Aartsma

Benjamin Leroy

Wopke van der Werf

Marcel Dicke

Erik H. Poelman

Felix J.J.A. Bianchi

Accepted for publication in Oikos 


\begin{abstract}
Chemical information influences the behaviour of many animals, thus affecting species interactions. Many animals forage for resources that are heterogeneously distributed in space and time, and have evolved foraging behaviour that utilizes information related to these resources. Herbivore-induced plant volatiles (HIPVs), emitted by plants upon herbivore attack, provide information on herbivory to various animal species, including parasitoids. Little is known about the spatial scale at which plants attract parasitoids via HIPVs under field conditions and how intraspecific variation in HIPV emission affects this spatial scale. Here, we investigated the spatial scale of parasitoid attraction to two cabbage accessions that differ in relative preference of the parasitoid Cotesia glomerata when plants were damaged by Pieris brassicae caterpillars. Parasitoids were released in a field experiment with plants at distances of up to $60 \mathrm{~m}$ from the release site using intervals between plants of $10 \mathrm{~m}$ or $20 \mathrm{~m}$ to assess parasitism rates over time and distance. Additionally, we observed host-location behaviour of parasitoids in detail in a semi-field tent experiment with plant spacing up to $8 \mathrm{~m}$. Plant accession strongly affected successful host location in field set-ups with $10 \mathrm{~m}$ or $20 \mathrm{~m}$ intervals between plants. In the semi-field set-up, plant finding success by parasitoids decreased with increasing plant spacing, differed between plant accessions, and was higher for host-infested plants than for uninfested plants. We demonstrate that parasitoids can be attracted to herbivore-infested plants over large distances $(10 \mathrm{~m}$ or $20 \mathrm{~m}$ ) in the field, and that stronger plant attractiveness via HIPVs increases this distance (up to at least $20 \mathrm{~m}$ ). Our study indicates that variation in plant traits can affect attraction distance, movement patterns of parasitoids, and ultimately spatial patterns of plant-insect interactions. It is therefore important to consider plant-trait variation in HIPVs when studying animal foraging behaviour and multitrophic interactions in a spatial context.
\end{abstract}




\section{Introduction}

Organisms are usually confronted with resources that are scattered across space and time. The spatial scale at which organisms forage for resources depends - besides the spatial distribution of resources - on species traits, such as the capacity for movement and the ability to acquire information about the resource distribution from a distance (Nathan et al., 2008). Organisms use information (cues), including visual, acoustic and olfactory cues to make foraging decisions. In addition, foraging decisions can be influenced by the animal's internal state, such as hunger level, and learning (Bowler \& Benton, 2005; Clobert et al., 2009). Compared to large animals, smaller animals such as insects may experience spatial scales differentially. Although smaller animals need to gather information on the availability of resources just like large vertebrates, they sometimes have a limited capacity for directed movement and have a short lifespan to acquire and use information (Wajnberg, 2006). For smaller animals, individual foraging behaviour is usually inferred from population patterns, leaving a knowledge gap on the possible use of information in individual foraging decisions (Holyoak et al., 2008). Yet, knowledge of how individual animals use information is important to understand at what spatial scale they interact with other individuals, both from the same and from other species.

A wide range of small animals have evolved foraging strategies based on olfactory cues (Raguso et al., 2015; Poelman \& Kos, 2016; Webster \& Cardé, 2016). Herbivore-induced plant volatiles (HIPVs), that are emitted by plants upon attack by herbivores, are remarkable olfactory cues in the sense that they provide indirect information on the presence of herbivores because they are produced by the plant under attack and not directly by the herbivores themselves (Vet \& Dicke, 1992; Hare, 2011; Turlings \& Erb, 2018). HIPVs and other plantderived volatiles are used during long-distance foraging by mammals (Finnerty et al., 2017), birds (Mäntylä et al., 2004; Amo et al., 2013; Mäntylä et al., 2014), reptiles (Stork et al., 2011), and insects (Turlings \& Erb, 2018). Yet, HIPV use is particularly well studied for parasitoid wasps (Vet \& Dicke, 1992; Turlings \& Erb, 2018). Parasitoids comprise an important and highly species-rich group of carnivorous insects which may regulate herbivore populations (Forbes et al., 
2018). They lay eggs and develop inside or on a host insect, and therefore their ability to find these hosts is closely linked to their fitness (Thiel \& Hoffmeister, 2009).

Because HIPVs provide information to parasitoids that in turn suppress herbivore attack, HIPVs can be considered a part of a plant's information-mediated indirect defences (Kessler \& Heil, 2011). Despite advances in the understanding of the role of HIPVs in parasitoid foraging behaviour, knowledge on the distance at which HIPVs are used by parasitoids is limited (Aartsma et al., 2017). Consequently, just like for chemical cues in general (Zimmer \& Zimmer, 2008), we have little understanding of the significance of plant volatiles in ecological processes at larger spatial scales under field conditions. With increasing distance from the source, a volatile blend will be diluted (Cardé \& Willis, 2008) and blend components differentially degrade under the influence of environmental factors, such as UV or ozone (Blande et al., 2014). Therefore, it is important to assess at what distances parasitoids interact with plants through HIPVs. While HIPVs are commonly referred to as long-distance cues, the spatial scale of 'long-distance' is under debate. Some studies report only very localized effects of HIPVs on insect distributions, less than $1.5 \mathrm{~m}$ away from the release point (Mallinger $e t$ al., 2011; Rodriguez-Saona et al., 2011). Others have recorded effects at larger spatial scales, up to 8 or $15 \mathrm{~m}$ (Bernasconi Ockroy et al., 2001; Braasch \& Kaplan, 2012a). Insight into the distance at which HIPVs attract parasitoids is crucial for our understanding of movement patterns as a result of host-location behaviour of parasitoids, both in nature and in agroecosystems. Such insights will reveal the spatial scale at which herbivore-infested plants interact with the enemies of the herbivores, and will improve our understanding of the spatial processes underlying population and community ecology (Mills \& Wajnberg, 2008). There is considerable variation within and between plant species in HIPV release (Degen et al., 2004; Gols et al., 2011). The identity of the herbivore, severity of attack, or presence of multiple herbivores may affect the strength and composition of HIPV blends, resulting in a dynamic and complex environment for parasitoids to locate their hosts (Dicke et al., 2009; de Rijk et al., 2013). It is well established that parasitoids prefer volatiles from damaged plants over those from non-damaged plants (Geervliet et al., 1996; Gols et al., 2012), but 
in a no-choice situation or combined with non-host plant species, parasitoids will also visit undamaged plants (Gohole et al., 2005; Pareja et al., 2007; Moraes et al., 2008). The effects of variation in plant-species traits have generally been studied in choice tests under controlled conditions. Preference for a certain plant accession can lead to higher parasitism rates in the field (Poelman et al., 2009b), although differences in field parasitism on different varieties cannot always be explained by volatiles (Degen et al., 2012). It is unknown whether plants whose HIPVs are preferred by parasitoids can be detected from a larger distance than HIPVs from less preferred plants.

The objective of the present study was to assess the effect of plant-trait variation in terms of HIPV emission on the spatial scale of parasitoid attraction. We made use of our earlier findings on relative preference of the parasitoid Cotesia glomerata to HIPVs for white cabbage, Brassica oleracea, accessions (from here on: cabbage accessions) (Poelman et al. 2009b). When damaged by Pieris brassicae caterpillars, the parasitoid's host, the cabbage accession Christmas Drumhead is preferred by C. glomerata parasitoids over the accession Badger Shipper in both wind tunnel assays (1 m distance) and field plots (Poelman et al. 2009b). We hypothesized that parasitism rates decline with increasing distance between plants and that differential preference of parasitoids (here called attractiveness of plants), as recorded in the laboratory (Poelman et al. 2009b), results in differences in spatial ecology among the cabbage accessions under field conditions. We tested these hypotheses in a field experiment using different spatial arrangements (10 m or 20 $\mathrm{m}$ spacing) of cabbage plants that differed in attractiveness, and in a semi-field tent experiment (spacing up to $8 \mathrm{~m}$ ) with the same system where we observed the behaviour of parasitoids in more detail and at a smaller spatial scale. These experiments provide novel information about the spatial scale at which plants interact with parasitoids that attack herbivores. We discuss the importance of plant genetic variation in volatile emission in the context of spatial scales, and the implications for foraging behaviour decisions based on HIPVs in the field. 


\section{Materials and Methods}

\section{Insects and plants}

Seeds of two white cabbage accessions (Brassica oleracea var. alba L.), the accession 'Badger Shipper' and the accession 'Christmas Drumhead', were obtained from the Centre for Genetic Resources (CGN-Wageningen, The Netherlands). Plants were sown in peat soil cubes in a greenhouse (L16:D8 photoperiod, at 18-26 ${ }^{\circ} \mathrm{C}$ and $40-70 \%$ relative humidity) and seedlings of three weeks old were placed outside in fine-meshed insect screen cages to acclimatize to field conditions. Five-week-old potted plants were used in the field experiments.

Caterpillars of Pieris brassicae L. (Lepidoptera: Pieridae) and adults of Cotesia glomerata L. (Hymenoptera: Braconidae) parasitoids were obtained from cultures at the laboratory of Entomology, Wageningen University. Pieris brassicae is a specialist herbivore of plants in the family Brassicaceae (Edger et al., 2015). Caterpillars were reared on Brussels sprouts plants (Brassica oleracea L. var. gemmifera cultivar Cyrus) in a greenhouse compartment (L16:D8 photoperiod, at $21 \pm 1{ }^{\circ} \mathrm{C}$ and $50-70 \%$ relative humidity). Cotesia glomerata is a small larval parasitoid specializing on P. brassicae and measures only 2-3 millimetres in size (Brodeur et al., 1996). Parasitoids were reared on $P$. brassicae caterpillars under similar greenhouse conditions. Cocoons were placed in a cage $(30 \times 30 \times 30 \mathrm{~cm}$, Bugdorm, Taiwan $)$ and the emerged adult parasitoids were allowed to mate and were fed with honey and water. The cage was kept in a climate cabinet at $24 \pm 1{ }^{\circ} \mathrm{C}, \mathrm{LD} 12: \mathrm{D} 12$. We used 2-4 days-old adult female parasitoids in the experiments.

\section{Wagon-wheel experiment}

The effect of Brassica accession and spacing between plants on host plant finding of parasitoids was studied in the field, using two fields of perennial rye-grass (Lolium perenne L.) and a spatial set-up of caterpillar-infested cabbage plants in each field that had the shape of a wagon wheel (Fig. 1). The two fields were situated near Wageningen, The Netherlands, and about $200 \mathrm{~m}$ apart. No brassicaceous plants were present in the direct vicinity of the fields. Parasitoids were released in a 3 by $3 \mathrm{~m}$ square of $5 \times 5$ cabbage plants at $75 \mathrm{~cm}$ plant distance, in the centre of each wagon wheel that was surrounded by a wired 
metal fence to exclude rabbits. Five transects (i.e. 'spokes') were laid out, radiating from the release area at $72^{\circ}$ angles. Each transect consisted of six single cabbage plants spaced $10 \mathrm{~m}$ apart, spanning a total length of $60 \mathrm{~m}$ from the centre. Each potted cabbage plant was placed in a hole in the ground and was also fenced with metal wire. During each replicate one 'Badger Shipper' wagon wheel and one 'Christmas Drumhead' wagon wheel were established, and accessions were alternated in each field in subsequent measurement sessions ('replicates') to prevent location bias.

To test whether the distance between infested plants affects parasitoid host finding, the plants in the 5 spokes were either all infested (full infestation with $10 \mathrm{~m}$ distance between infested plants) or infested alternately within each spoke (half infestation with $20 \mathrm{~m}$ distance between infested plants). Replicates with distances of 10 or $20 \mathrm{~m}$ between infested plants on the spokes of the wagon wheels were alternated over time to realize a total of four replicate replicates with

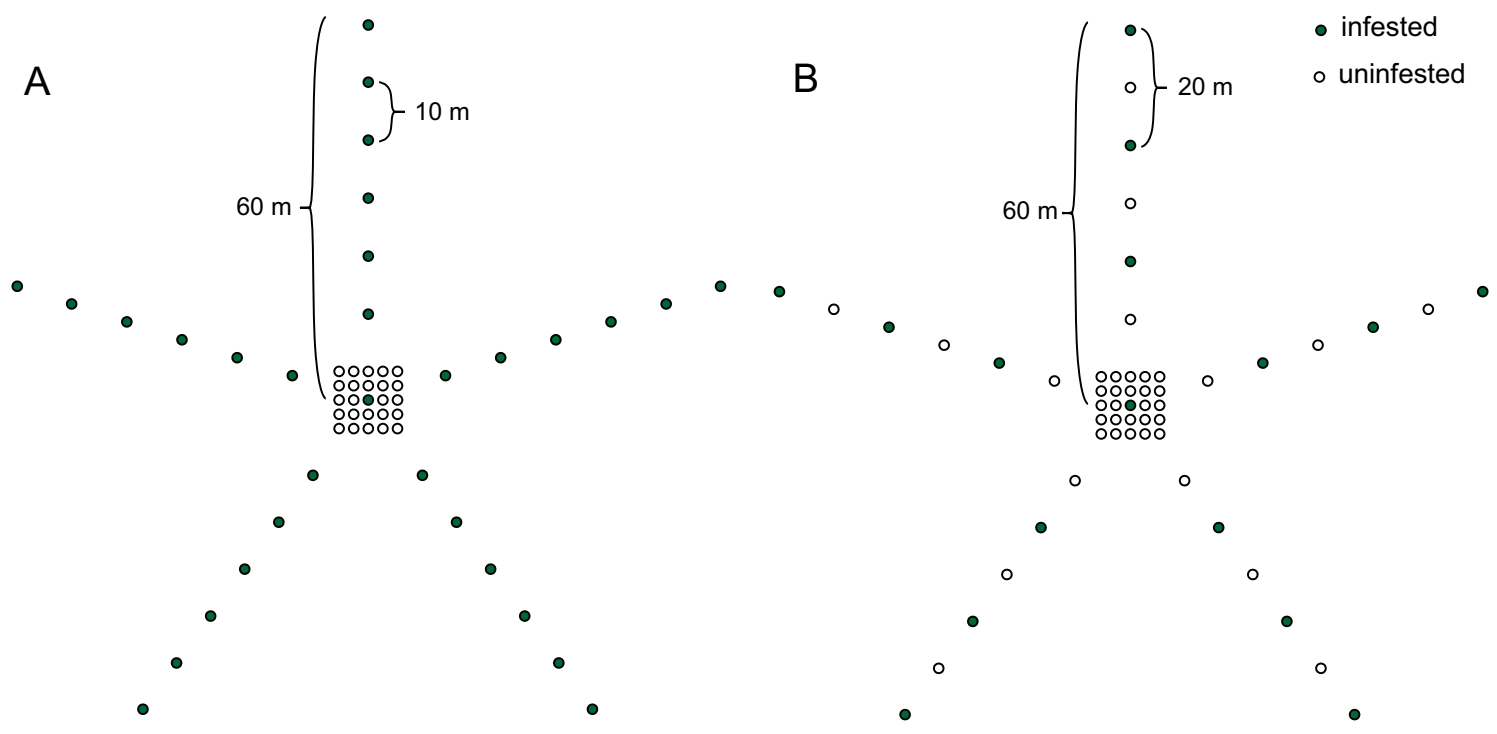

Figure 1: Spatial arrangement of wagon-wheel experiment. White cabbage plants (Brassica oleracea var. alba L.) of the attractive accession 'Christmas Drumhead' or the less attractive 'Badger Shipper' were placed in a homogeneous grassland. A central plot with $5 \times 5$ cabbage plants at $75 \mathrm{~cm}$ plant distance was created, from which five transects were laid out by placing six cabbage plants at $10-\mathrm{m}$ intervals. Twentyfive mated Cotesia glomerata females were released from the central plant. In design A, each of the six plants on each transect was infested with 10 caterpillars of Pieris brassicae, the host of $C$. glomerata, while in design B, only every second plant (i.e. at $20 \mathrm{~m}, 40 \mathrm{~m}$ and $60 \mathrm{~m}$ ) was infested with caterpillars, while the other plants (at $10 \mathrm{~m}, 30 \mathrm{~m}$ and $50 \mathrm{~m}$ ) were not infested. 
$10 \mathrm{~m}$ distance between infested plants and four replicate replicates with $20 \mathrm{~m}$ distance between infested plants. Replicates were done from May to September 2014. The grass fields were mown three times during this period in weeks that the fields were empty.

At the beginning of each replicate, potted cabbage plants were planted in the centre and the spokes of each wagon wheel. The middle plant of the central $5 \times 5$ plot was infested with 15 L1 P. brassicae caterpillars, while the rest of the plot remained uninfested. To verify that parasitism in our experiments was not caused by naturally occurring parasitoids or parasitoids released in previous experimental replicates, we assessed background levels of parasitism at the start of each replicate by introducing and recollecting the first batch of caterpillars on each plant in the spokes of the wheel $24 \mathrm{~h}$ before releasing the parasitoids. Subsequently, 25 female $C$. glomerata parasitoids were released from plastic tubes on the ground near the stem of the central plant. Infested plants in transects received 10 L1 P. brassicae caterpillars per plant. During four consecutive days, all caterpillars were recollected and replaced with new caterpillars each day. Caterpillars on the central plant, which were used to stimulate parasitoid hostsearching behaviour, were removed 24 hours after releasing the parasitoids and not replaced. In three earlier pilot trials, where 10 parasitoids were released and no caterpillars were present on the central plant, no parasitism was found. Recollected caterpillars were dissected under a stereomicroscope to record the presence of C. glomerata eggs. All cabbage plants were removed and the field was left empty for a week, after which the next replicate started.

\section{Tent experiment}

The influence of Brassica oleracea accession and spacing between infested plants on host finding by C. glomerata was studied in more detail and at a smaller spatial scale in a tent experiment. A large mesh tent $(16 \mathrm{~m} \times 8 \mathrm{~m} \times 2.5 \mathrm{~m})$ was set-up in a mown monoculture field of perennial rye grass near Wageningen. Infested or uninfested Badger Shipper or Christmas Drumhead plants were introduced at $1 \mathrm{~m}, 2 \mathrm{~m}, 4 \mathrm{~m}$ or $8 \mathrm{~m}$ in the upwind direction from an uninfested plant where C. glomerata parasitoids were released. Infested plants were inoculated with $10 \mathrm{~L} 1$ P. brassicae caterpillars $24 \mathrm{~h}$ before the start of the experiment to induce HIPV production. 
Female parasitoids were brought to the tent in a small mesh cage that included honey as food source and water. Parasitoids were stimulated to search for hosts by a short exposure to a Brussels sprouts leaf with P. brassicae caterpillars. Thereafter, we released five parasitoids near the stem of the release plant. To differentiate between parasitoids, four out of each five were marked a day prior to the observations with nail polish (Hema) of different colours. Parasitoids that were visibly wounded after marking were discarded. Unmarked parasitoids were used as a control to assess whether marking had an effect on foraging behaviour. Each parasitoid was only used once for the experiment. Twenty parasitoids were observed for each combination of accessions (Badger Shipper or Christmas Drumhead), distance between plants ( $1 \mathrm{~m}, 2 \mathrm{~m}, 4 \mathrm{~m}$ or $8 \mathrm{~m})$, and infestation with $P$. brassicae caterpillars (yes or no), for a total of 320 parasitoids.

Times until flight initiation and arrival at the (un)infested plant were recorded by visual examination of the release plant and target plant. Parasitoids that (i) did not initiate flight within 30 minutes, or (ii) did not arrive within $30 \mathrm{~min}, 45$ $\mathrm{min}, 60 \mathrm{~min}$ or $70 \mathrm{~min}$ on infested plants at distances of $1 \mathrm{~m}, 2 \mathrm{~m}, 4 \mathrm{~m}$ and $8 \mathrm{~m}$, respectively, were considered non-responsive and were excluded from analyses. We used new sets of control and infested plants on each day, and accessions were tested in alternate weeks. Observations were conducted on dry days with temperatures ranging from $17-25^{\circ} \mathrm{C}$ in July and August 2014.

\section{Statistical analysis}

Wagon-wheel experiment

Background parasitism was found in one replicate of the wagon wheel experiment, which was excluded from the analysis. The final dataset included data from three full infestation replicates and four half infestation replicates for each cabbage accession.

Host-plant finding by C. glomerata was analysed as presence/absence data. If eggs of C. glomerata were found in at least one of the recollected caterpillars of a plant at a location on the spokes of the wagon wheel, parasitism at this location was recorded as 'present'. We used a generalized linear mixed model (GLMM) with binomial error distribution and plant accession (categorical, levels: 
Christmas Drumhead and Badger Shipper), spatial arrangement (categorical, levels: $10 \mathrm{~m}$ and $20 \mathrm{~m}$ ), distance from central plot (continuous) and day after release (continuous variable) as fixed effects. The factors "week" (i.e. week in which the replicate was held) and "plant ID" (as each plant was used for four consecutive days) were included as random effects. Three-way and higher term interactions were not fitted because the biological interpretation of such interactions is problematic. A full model containing all fixed variables with twoway interactions and random effects was the starting point for model selection using the dredge procedure in the $\mathrm{R}$ package MuMIN. This procedure results in a complete set of sub-models with combinations of the terms of the full model, and sorts the sub-models based on the corrected Akaike Information Criterion (AICc). Models with a $\triangle \mathrm{AICc}$ of $<2$ have a substantial support from the data. We present tables for the simplest model of the sub-models with substantial support, with the fewest degrees of freedom. The full list of sub-models and the selected variables is presented in the Supplementary Information.

To identify significant interactions, a Tukey post-hoc test for the GLMM was used to compare combinations of factors. All analyses were conducted in $\mathrm{R}$ using the statistical packages 'Ime4' (Bates et al., 2015), 'MuMIN' (Barton, 2016), 'multcomp' (Hothorn et al., 2008) and 'car' (Fox \& Weisberg, 2011).

\section{Tent experiment}

Parasitoids that did not initiate flight were excluded from the analysis. Landing success was analysed as a binomial response variable. Parasitoids that landed on the infested plant within the time limit were recorded as a success, and other parasitoids as a failure. We used a generalized linear model with binomial error distribution and infestation treatment (categorical, levels: infestation and no infestation), distance from release point (continuous variable), plant accession (categorical, levels: Christmas Drumhead or Badger Shipper), and marking (categorical, levels: marked and non-marked) as fixed effects. The full model containing all these variables and two-way interactions was used for model selection with the dredge procedure as described above. 
Search time of C. glomerata parasitoids was analysed for parasitoids that successfully found infested plants within the time limit. We used a general linear model with searching time as response variable and accession, distance, marking, and infestation treatment as fixed effects. Search time was square-root transformed to meet normality of residuals and equality of variance requirements. Model selection was conducted as described for the wagon-wheel experiment.

\section{Results}

\section{Wagon-wheel experiment}

A total of 12,000 P. brassicae caterpillars were placed in the field during the season and 7,090 (59\%) of these were recovered. Recovery rate was similar to previous studies using the same methodology (Poelman et al. 2009b). Overall, C. glomerata parasitism rate of recovered caterpillars was $9.7 \%(n=790)$ and ranged from $0 \%$ to $30 \%$ of recovered caterpillars in the respective replicates (Supplementary Information Fig. 1; Supplementary Information Fig. 2).

Model selection (Supplementary Information Table 1) indicated that host-plantfinding was influenced by accession, spacing $(10 \mathrm{~m}$ or $20 \mathrm{~m}$ between P. brassicaeinfested plants), distance from release point, and days after release; there was a significant interaction between accession and spatial arrangement (Table 1). Host-plant finding was negatively associated with distance from the parasitoid release point and the number of days after release (Table 1).

In setups with $10 \mathrm{~m}$ spacing between infested plants, accession identity had no effect on host plant finding (Fig. 2, Tukey post hoc, $z=1.709, p=0.28$ ). There was no difference in host-plant finding between setups of Christmas Drumhead (attractive) with $10 \mathrm{~m}$ or $20 \mathrm{~m}$ spacing (Tukey post hoc, $z=-0.540, p=0.94$ ) and between setups of Badger Shipper (less attractive) with $10 \mathrm{~m}$ spacing and Christmas Drumhead with $20 \mathrm{~m}$ spacing (Tukey, $z=-0.079, p=1.0$ ). In setups with $20 \mathrm{~m}$ spacing between infested plants, fewer plants with parasitized caterpillars were found in wagon wheels with the less attractive Badger Shipper accession than in those with the attractive Christmas Drumhead accession (Fig. 
Table 1: Simplest generalized linear mixed model ( $<2 \Delta \mathrm{AICc}$ and lowest $d f$ ) for host-plant finding success by Cotesia glomerata parasitoids in the wagon wheel with estimates, standard error (SE) of estimates, Wald's z-statistic and corresponding $p$-value. Significant $p$-values are highlighted in bold $(a=0.05)$. The less attractive accession Badger Shipper and $10 \mathrm{~m}$ are reference treatments for accession and host spacing, respectively.

Fixed effect

Intercept

Accession Christmas Drumhead (categorical)

$10 \mathrm{~m}$ host spacing (categorical)

Distance from release point (continuous)

Time since release (continuous)

Accession $\times$ host spacing (categorical)

$\begin{array}{llll}\text { Estimates } & \text { SE } & \text { Z-value } & p \\ 0.363 & 0.906 & 0.401 & 0.69 \\ 0.519 & 0.312 & 1.664 & 0.10 \\ -3.407 & 1.299 & -2.623 & 0.009 \\ -0.050 & 0.008 & -5.800 & <0.001 \\ -0.016 & 0.006 & -2.688 & 0.007 \\ 2.815 & 0.798 & 3.530 & <0.001\end{array}$

- Christmas Drumhead

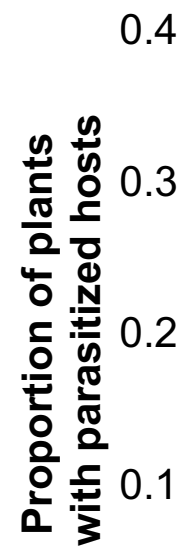

0

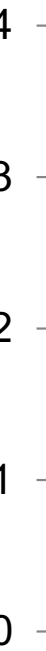

a

Badger Shipper

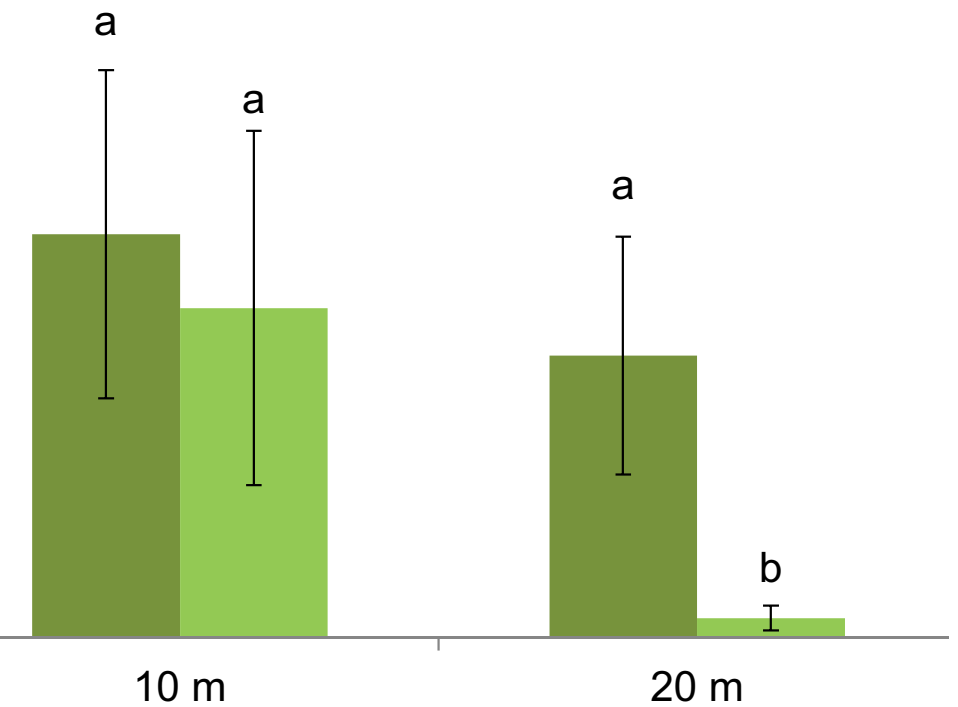

\section{Distance between caterpillar-infested plants}

Figure 2: Proportion of Pieris brassicae-infested cabbage plants found by Cotesia glomerata parasitoids for the attractive accession Christmas Drumhead and less attractive accession Badger Shipper in $10 \mathrm{~m}$ and $20 \mathrm{~m}$ plant-spacing wagon-wheel arrangements (Fig. 1). Bars and error bars represent averages across replicates and SEM, respectively ( $N=3$ for each accession in $10 \mathrm{~m}$ plant spacing, $N=4$ for each accession in $20 \mathrm{~m}$ plant spacing). Different letters indicate significant differences between all bars $(p<0.05)$ based on pair-wise Tukey LSD post hoc tests. 
2; Tukey post hoc, $z=4.521, p<0.001)$. Caterpillars were parasitized at a distance of $60 \mathrm{~m}$ after a time interval of $24 \mathrm{~h}$ after release (Fig. 2 and Supplementary Information 1 Fig. 1; Supplementary Information Fig. 2).

\section{Tent experiment}

Out of the 320 parasitoids observed in the tent experiment, 127 parasitoids $(39.7 \%)$ successfully landed on the experimental plant within the time limit, while $53(16.5 \%)$ did not initiate flight within 30 minutes. The others (43.8\%) initiated flight but did not arrive on the experimental plant within the time limit.

Host-plant finding success by C. glomerata was influenced by accession, distance from release point and whether the plant was infested with $P$. brassicae caterpillars or not (Supplementary Information Table 2). Marking of parasitoids had no effect

Table 2: Simplest generalized linear model ( $<2 \Delta \mathrm{AICc}$ and lowest $\mathrm{df}$ ) for host plant finding success by Cotesia glomerata parasitoids in a tent experiment with estimates, standard error (SE) of estimates, Wald's $z$-statistic and corresponding $p$-value. Significant $p$-values are highlighted in bold $(\alpha=0.05)$. The less attractive accession Badger Shipper and no infestation with caterpillars were the reference treatments for accession and infestation with caterpillars, respectively. $\mathrm{N}=267$ parasitoids.

$\begin{array}{lccrr}\text { Fixed effect } & \text { Estimates } & \text { SE } & \text { Z-value } & p \\ \text { Intercept } & -0.121 & 0.286 & -0.421 & 0.674 \\ \text { Accession Christmas Drumhead (categorical) } & 0.728 & 0.260 & 2.806 & 0.005 \\ \text { Distance from release point (continuous) } & -0.169 & 0.050 & -3.416 & <0.001 \\ \text { Infestation with caterpillars (categorical) } & 0.638 & 0.259 & 2.469 & 0.014\end{array}$

Table 3: Simplest general linear model ( $<2 \Delta \mathrm{AICc}$ and lowest $\mathrm{df}$ ) for square root transformed search time (s) by Cotesia glomerata parasitoids in a tent experiment with estimates, standard error (SE) of estimates, t-statistic and corresponding $p$-value. Significant $p$-values are highlighted in bold ( $\alpha=0.05)$. No infestation with caterpillars was the reference treatment for infestation with caterpillars. $N=127$ parasitoids.

$\begin{array}{lrrrr}\text { Fixed effect } & \text { Estimates } & \text { SE } & t \text {-value } & p \\ \text { Intercept } & 30.165 & 2.488 & 12.125 & <0.001 \\ \text { Distance from release point (continuous) } & 2.637 & 0.398 & 6.625 & <0.001 \\ \text { Infestation with caterpillars (categorical) } & -6.325 & 2.075 & -3.048 & 0.003\end{array}$


A $\quad$ Uninfested $\square$ Infested

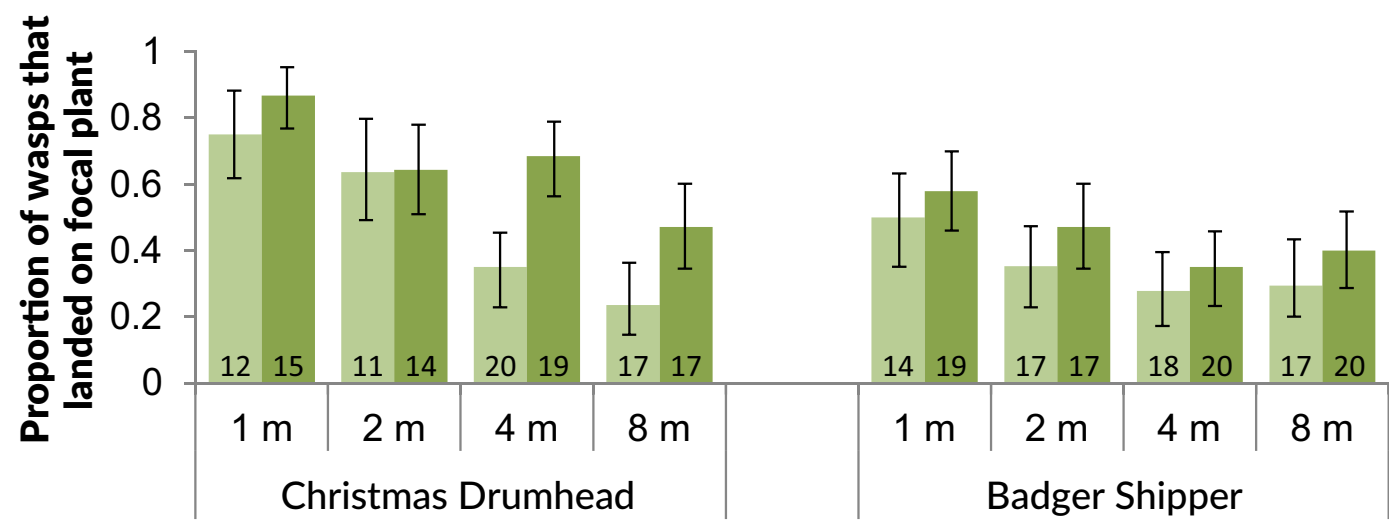

B

Uninfested $\quad$ Infested

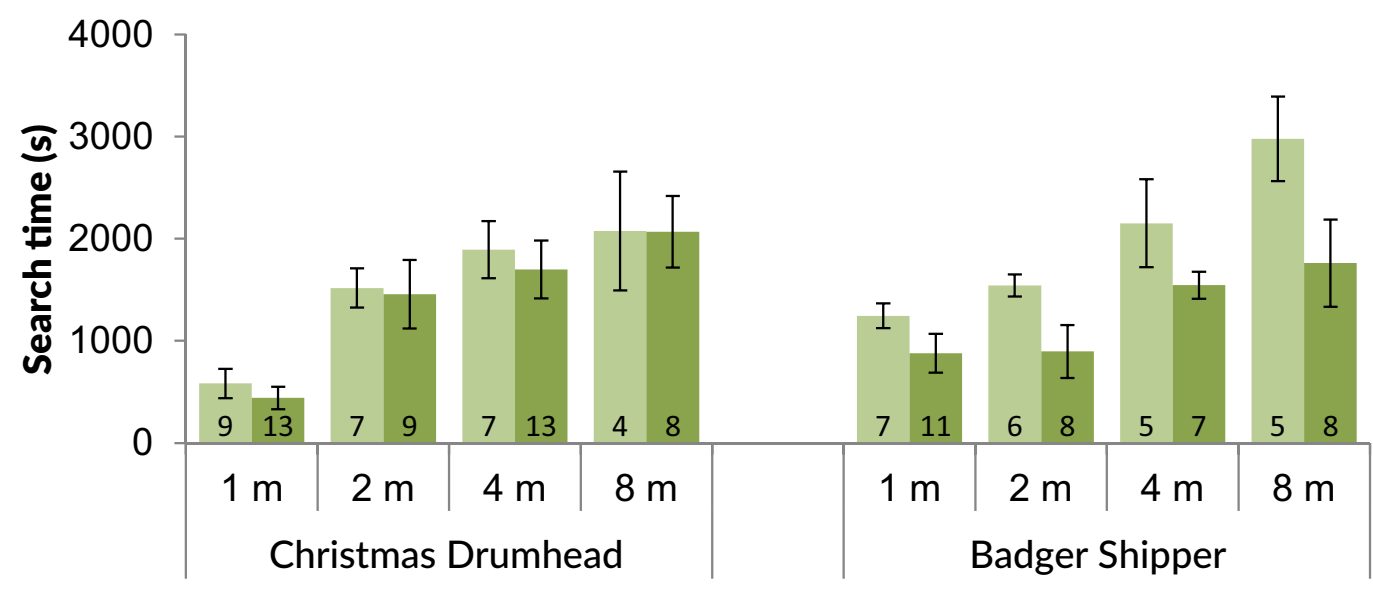

Figure 3: Proportion host location success (A) and search time of successful wasps (B) of Cotesia glomerata parasitoids when challenged to locate a focal white cabbage plant (Brassica oleracea var. alba L.) of either the attractive accession Christmas Drumhead or the less attractive accession Badger Shipper placed at different distances from the release plant $(1 \mathrm{~m}, 2 \mathrm{~m}, 4 \mathrm{~m}$ or $8 \mathrm{~m})$. Colours indicate whether the plant was infested for $24 \mathrm{~h}$ with $10 \mathrm{~L} 1$ Pieris brassicae caterpillars or not. For (A), bars and error bars represent proportion of successful landings and binomial standard deviation, respectively. For (B), bars and error bars represent averages and SEM, respectively. The number at the base of the bars indicates the number of parasitoids which initiated flight after release and the number of parasitoids which landed on the plant in figure a and b respectively. 
on host-plant finding success (Table 2). Parasitoids were more often successful in finding plants near the release point than plants further away, and more parasitized hosts were found on plants of the attractive Christmas Drumhead accession than on plants of the less attractive Badger Shipper accession (Table 2, Fig. 3). The infestation of the focal plant with caterpillars also led to higher host-plant finding success on both accessions as compared to plants that did not contain caterpillars (Table 2, Fig. 3).

Search time of C. glomerata was influenced by distance from the release point and infestation with $P$. brassicae caterpillars. Parasitoids took more time to land on plants that were further away from the release point (Table 3, Fig 3): the shortest search time was 16 seconds to find a Christmas Drumhead plant at 1 $m$ distance and the longest search time was 4531 seconds to find an uninfested Badger Shipper plant a $8 \mathrm{~m}$ distance. Search time of the parasitoids was lower when plants were infested with $P$. brassicae (Table 3, Fig 3). The interaction between accession and infestation was also selected in some models, as was the main effect of marking of the parasitoids (Supplementary Information Table 3). Marking and accession did not influence search time.

\section{Discussion}

Animal behaviour is at the basis of many species interactions and, thus, at the basis of ecological dynamics (Sih et al., 2012). Animal behaviour is guided by information and chemical information is an important source of cues for foraging animals (Raguso et al., 2015). Chemical information as conveyed by volatile chemicals mediates interactions between organisms before they actually meet. Thus, volatile chemicals enlarge the interaction space. Intraspecific variation in the emission of volatile chemicals may result in intraspecific variation of species interactions with consequences for population dynamics and community ecology. Here, we investigated intraspecific variation in plant volatiles that are induced by insect herbivory and that attract the enemies of the inducing herbivores. Herbivore-induced plant volatiles consist of a complex blend of tens of chemical compounds produced by plants under herbivore 
attack, which provide important information to both vertebrate and invertebrate natural enemies of herbivores that forage for insect herbivores (Poelman \& Kos, 2016).

Our field study shows that accession identity does not only influence the preference of parasitoids at a close range (Poelman et al., 2009b), but also the distance from which parasitoids are recruited in field situations: the spatial scale of parasitoid attraction ranges from $10 \mathrm{~m}$ to $20 \mathrm{~m}$ for the less attractive cabbage accession to $20 \mathrm{~m}$ and likely beyond for the more attractive accession over the time scale of the experiment. The hosts of $C$. glomerata parasitoids, the lepidopteran P. brassicae, lays clutches of eggs and the emerging caterpillars feed as a group; to avoid competition, the butterflies distribute their egg batches patchily and distances between plants with hosts can easily be beyond $20 \mathrm{~m}$, but within the range of the current field experiment. While accession identity did not influence the attraction of parasitoids at $10 \mathrm{~m}$ plant spacing in the field experiment, observations of parasitoid behaviour revealed that host finding success at distances smaller than $10 \mathrm{~m}$ was higher for the attractive accession than for the less attractive accession in a semi-field tent setting. Parasitoid searching behaviour was further influenced by the distance between the plant and the release point, and presence of hosts on the focal plant. Parasitism rates declined with time after release and increasing distance from the centre of the wagon-wheel setup.

While the movement ecology and perceptual range of larger animals, such as ungulates, have been relatively well studied (e.g. Zollner and Lima, 2005; Fryxell et al. 2008), few studies have assessed the spatial scale of volatile emission and effects on the foraging behaviour or attraction of arthropods (Aartsma et al., 2017). Most field studies using HIPV blends assess the attraction of insects to a point source and quantify insect attraction at or close to the point source (reviewed by Aartsma, et al. 2017). While this provides information on which HIPV compounds or blends attract certain insects, it does not tell us from which distance these insects are attracted. With increasing distance from an odour source, an odour plume will be diluted and change direction as a result of wind fluctuation (Cardé \& Willis, 2008). Moreover, individual components 
of the blend are differentially susceptible to environmental degradation (Blande et al., 2014). While parasitoids have navigational behavioural strategies to cope with these fluctuations in the strength of odour plumes (Kaiser et al., 1994), a higher probability of losing the plume during flight orientation might result in a lower host-plant finding success at larger distances, as observed in the tent experiment. The dynamics of odour-plume composition over time and space can compromise host-finding success under field conditions, and this is likely to influence the information value.

The spatial scale of arthropod attraction to infochemicals has predominantly been studied using synthetic volatiles. Increasing the number of pheromone point sources resulted in antennal responses of the moth Plodia interpunctella at larger distances (up to $60 \mathrm{~m}$ ) downwind from these sources, suggesting that an increase in the number of volatile-emitting sources can lead to insect attraction from larger distances (Andersson et al., 2013). This may also apply to intraspecific variation in volatile-emission rates in plants, although we are not aware of any field electroantennogram (EAG) studies using plants as odour source. Applying vials with phenylethyl alcohol (as a synthetic volatile) led to both repellent and attractant effects on the abundance of insects from distances of at least $8 \mathrm{~m}$ (Braasch \& Kaplan, 2012). However, these studies did not assess whether a change in odour concentration influences the distance from which insects are recruited. Other field studies report attraction and aggregation of arthropods to methyl salicylate at only short distances $(<1.5 \mathrm{~m})$ from the point source (Mallinger et al., 2011; Rodriguez-Saona et al., 2011). Our study shows that, in field situations, parasitoids are able to find host-infested cabbage plants from distances up to $10 \mathrm{~m}$ and possibly beyond $20 \mathrm{~m}$ (ca 8000 parasitoid body lengths) within a day, depending on the HIPV profile of the accession. Future studies should identify the spatial scale of attraction in more complex settings, such as plant communities with host and non-host herbivore feeding, which are known to hamper parasitoid host location (de Rijk et al., 2013; Desurmont et al., 2014; Kostenko et al., 2015).

The attractive accession Christmas Drumhead attracted parasitoids from a larger distance than the less attractive accession Badger Shipper. Although the 
HIPV profiles of these accessions have been found to differ in quantitative and qualitative aspects under feeding damage by Pieris caterpillars (Poelman et al., 2009b), it remains to be identified whether qualitative or quantitative components explain the distance of parasitoid attraction. Nevertheless, the present study provides the first empirical evidence that the differences in HIPV emission by the two accessions result in different attraction distances in the field, which has consequences for the overall spatial scale of attraction of parasitoids by HIPVs. Theoretically, a doubling of the attraction distance may lead to a 2-to-4-fold increase in catchment area of parasitoids depending on whether parasitoids are attracted from only the upwind direction or from all directions. This study shows that parasitoids can spread within one day through the whole $60 \mathrm{~m}$ assemblage of cabbage plants when inter-plant distances are $10 \mathrm{~m}$ or less. This is particularly important for parasitoid species that specialize on particular host stages and therefore facing a limited window of opportunity to find a host in the right stage (van Nouhuys \& Ehrnsten, 2004).

Parasitism rates declined with time after release and increasing distance from the centre of the wagon-wheel setup. This may be explained by several non-exclusive mechanisms, including (i) mortality reducing the number of actively searching parasitoids in the field, (ii) parasitoids leaving the experimental field because of a lack of floral food resources or simply due to random movement, (iii) female parasitoids becoming egg limited, and (iv) increasing distance between the spokes of the wagon wheel design at further distance from the release site. In the absence of HIPV information, parasitoids are expected to fly in crosswind direction (Williams et al., 2007). However, in this case one would expect that with $20 \mathrm{~m}$ spacing between plants parasitoids may have encountered hostinfested plants of the less-attractive accession when moving cross-wind without sensing cues and then tracing HIPV cues when they moved closer to the plants. Instead, we found that the parasitism rate in the wagon-wheel setups with the less attractive accession with $20 \mathrm{~m}$ spacing was negligible. It is known that parasitoids may switch from foraging to dispersing mode when information on hosts is not perceived or hosts are not found. Parasitoids may disperse by upward flight and the use of wind currents at higher altitudes (Kristensen et al., 2013b; Schellhorn et al., 2014). However, the factors eliciting the switch from small-scale searching 
behaviour to passive dispersal are little understood and deserve further study. Future research on animal dispersal should take into account the availability of information, such as HIPVs or other cues, at different distances to better understand patterns of dispersal at the landscape scale.

The distance from which HIPVs attract natural enemies of herbivores is relevant in the context of information-mediated indirect defence by plants and biological control of insect pests. First, plant varieties with a larger attraction distance may have a higher chance of attracting the enemies of their attackers. Additionally, both natural enemy and host population dynamics might be influenced by the enemies' ability to detect host presence from a distance by increased attack rates. Second, in recent years there has been an increasing interest in manipulating plant traits, including indirect defence using HIPVs, to enhance biological control in agricultural fields (Cortesero et al., 2000; Kappers et al., 2011; Kaplan, 2012a; Penaflor \& Bento, 2013; Gish et al., 2015; Stenberg et al., 2015). There are, however, still many open questions on interactions of insects of different trophic levels with plants emitting HIPVs and the practical implementation of crops making use of increased indirect defences (Gish et al., 2015). In agricultural systems where monocultures cover large areas, it is important to consider from what distance HIPVs attract natural enemies and whether landscape features can supply these enemies and thus affect parasitism in the field. While our findings suggest that attractive accessions can attract larger parasitoid numbers because of an extended volatile "catchment area", further work is needed to show how attraction by HIPVs can be exploited to increase parasitism rates in commercial agricultural field situations. Further studies are also needed to explore the implications of HIPV release for tritrophic interactions and effectiveness of biological control at a landscape level.

Insight in spatial ecology is important to understand population dynamics and community dynamics. Interactions between individuals, such as herbivoreplant, predator-prey, and pollinator-flower interactions, are at the basis of these processes. However, these interactions extend far beyond the physical interaction between the individual. Individuals emit cues that can influence the behaviour of others. Here, we have shown that a small insect of ca $2-3 \mathrm{~mm}$ small can interact 
with plants on which her hosts feed at a distance of 8000 body lengths. Thus, indirect cues produced by plants in response to herbivory have a large influence on the spatial scale at which parasitoids interact with herbivores. Moreover, this extension of the spatial interaction between two animal species is dependent on intraspecific variation among plant individuals. Investigating the consequences of the largely extended spatial scale of interactions between small animals for population dynamics and community dynamics will be an important challenge for future studies.

\section{Acknowledgements}

We thank Dine Volker and the staff of Unifarm for their help in the field work, and the rearing staff of the Laboratory of Entomology for the insects used in this experiment. 


\section{Supplementary Information}
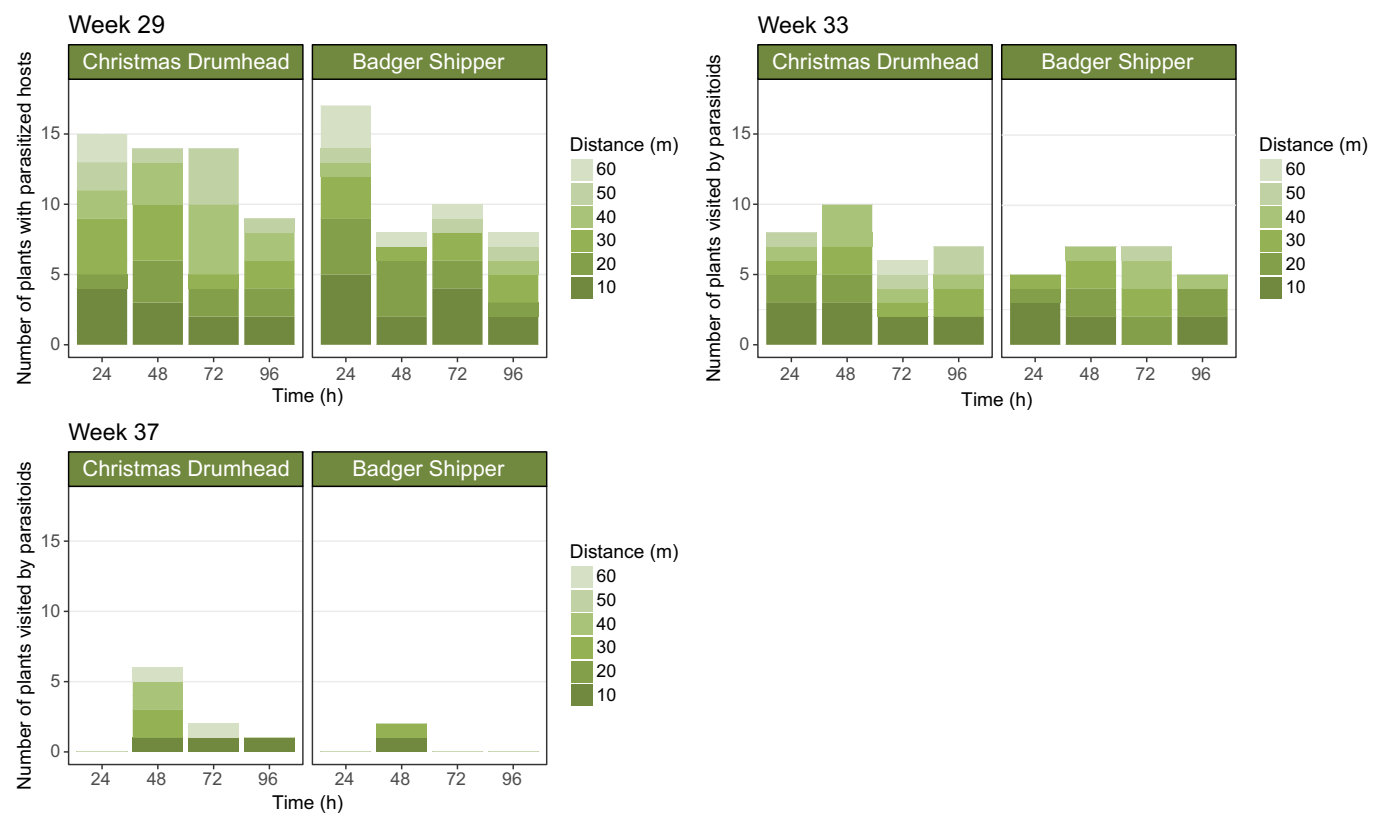

Figure 1: Number of white cabbage plants (Brassica oleracea var. alba L.) with Pieris brassicae caterpillars parasitized by Cotesia glomerata in experimental setups (wagon wheels) with $10 \mathrm{~m}$ between the infested plants. Week indicates the week of the year in which the replicate was conducted and results are shown for the attractive accession Christmas Drumhead and the less attractive accession Badger Shipper. Lighter colours indicate greater distance from the central release point and bars show data for the respective collection dates after release of the parasitoids. 

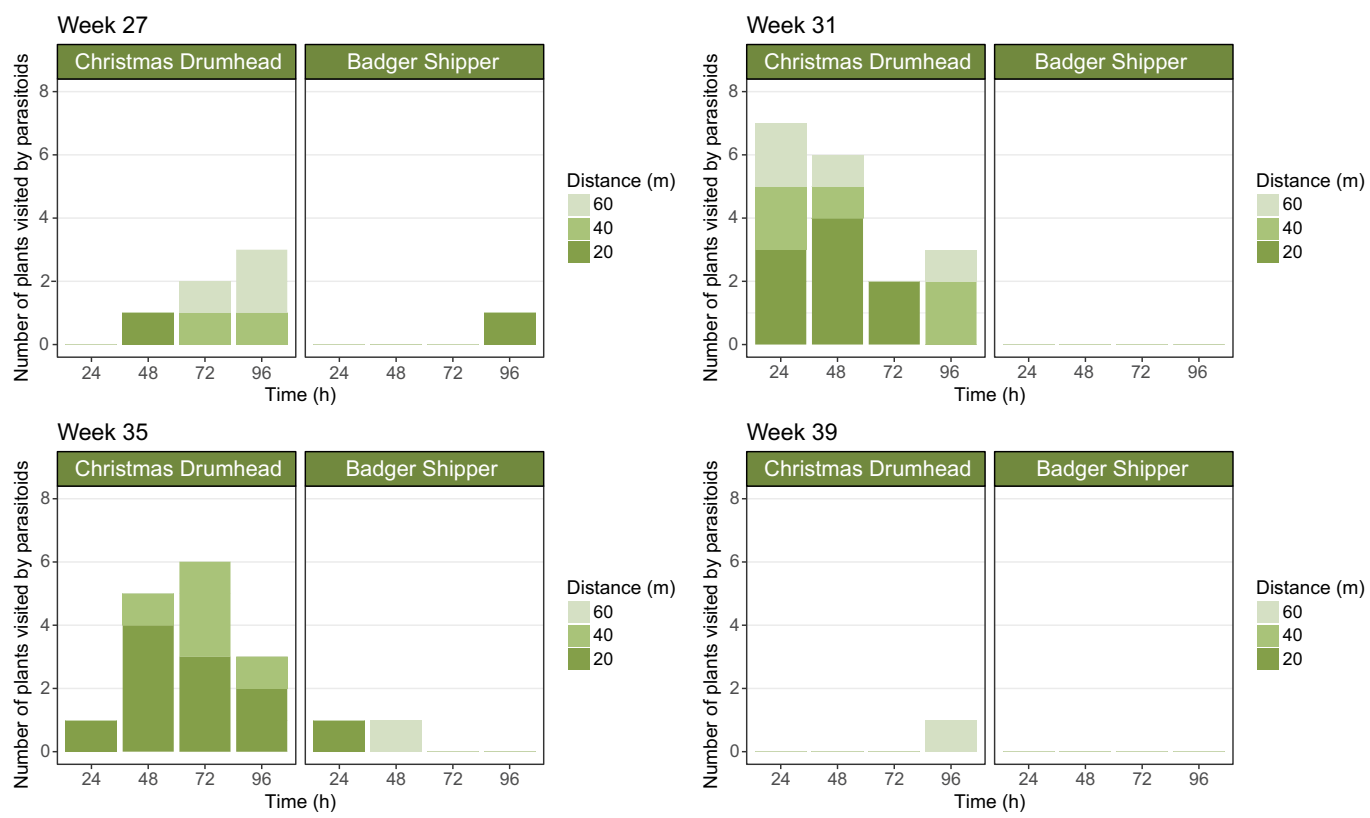

Figure 2: Number of white cabbage plants (Brassica oleracea var. alba L.) with Pieris brassicae caterpillars parasitized by Cotesia glomerata in experimental setups (wagon wheels) with $20 \mathrm{~m}$ between the infested plants. Week indicates the week of the year in which the replicate was conducted and results are shown for the attractive accession Christmas Drumhead and the less attractive accession Badger Shipper. Lighter colours indicate greater distance from the central release point and bars show data for the respective collection dates after release of the parasitoids. 
Table 1: Overview of model selection results for host plant finding of Cotesia glomerata in the wagon wheel experiment. Explanatory variables included accession (ac), distance from central release area (dst), time after release (tim), plant spacing (trm) and two-way interactions between these variables. Selected variables are shown as a "•". Models are ranked based on the Akaike Information Criterion corrected for finite sample sizes (AICc). Only models with substantial support from the data are presented $(\triangle \mathrm{AICc} \leq 2)$.

\begin{tabular}{|c|c|c|c|c|c|c|c|c|c|c|c|c|c|c|}
\hline (int) & $a c$ & $d s t$ & tim & trm & $a c^{*} d s t$ & actim & $a c^{*}$ trm & $d s t^{*} t i m$ & $d s t^{*}$ trm & tim*trm & $d f$ & $A I C C$ & $\triangle A I C c$ & weight \\
\hline 0.36280 & 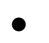 & - & 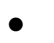 & $\bullet$ & & & $\bullet$ & & & & 10 & 809.0 & 0.00 & 0.202 \\
\hline 3900 & • & & & • & $\bullet$ & & $\bullet$ & & & & 11 & 809.3 & 0.26 & 0.174 \\
\hline 46510 & $\bullet$ & $\bullet$ & & 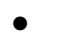 & & & $\bullet$ & & & $\bullet$ & 11 & 809.9 & 0.95 & 0.129 \\
\hline 68200 & $\bullet$ & $\bullet$ & 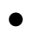 & 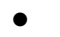 & & & - & - & & & 11 & 810.2 & 1.17 & 0.111 \\
\hline 88710 & $\bullet$ & $\bullet$ & $\bullet$ & • & $\bullet$ & & $\bullet$ & & & $\bullet$ & 12 & 810.3 & 1.26 & 0.106 \\
\hline 2800 & • & $\bullet$ & 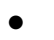 & • & - & & • & $\bullet$ & & & 12 & 810.4 & 1.39 & 0.100 \\
\hline 47370 & - & $\bullet$ & & & & & & & 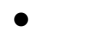 & & 11 & 810.6 & 1.56 & 0.091 \\
\hline 50020 & $\bullet$ & • & $\bullet$ & $\bullet$ & & $\bullet$ & • & & & & 11 & 810.7 & 1.74 & 0.086 \\
\hline
\end{tabular}

Table 2: Overview of model selection statistics for models predicting host plant finding of Cotesia glomerata. Explanatory variables included were accession (ac), distance from release plant (dst), marking of parasitoid (mrk), infestation of plant with Pieris brassicae caterpillars (trm) and two-way interactions between these variables. Selected variables are shown as a "•". Models are ranked based on the Akaike Information Criterion corrected for finite sample sizes (AICC). Only models with substantial support from the data are presented $(\triangle \mathrm{AICc} \leq 2)$.

\begin{tabular}{|c|c|c|c|c|c|c|c|c|c|c|c|}
\hline (int) & $a c$ & $d s t$ & mrk & trm & $a c^{*} d s t$ & $a c^{*}$ trm & $d s t^{*}$ trm $a c^{*} d s t^{*}$ trm & $d f$ & $\mathrm{AICC}$ & $\triangle A I C C$ & weight \\
\hline-0.1925 & $\bullet$ & $\bullet$ & $\bullet$ & $\bullet$ & $\bullet$ & & & 6 & 351.2 & 0.00 & 0.143 \\
\hline-0.3911 & $\bullet$ & $\bullet$ & & $\bullet$ & $\bullet$ & & & 5 & 351.8 & 0.63 & 0.105 \\
\hline 0.0801 & $\bullet$ & $\bullet$ & $\bullet$ & $\bullet$ & & & & 5 & 351.9 & 0.69 & 0.102 \\
\hline-0.1205 & $\bullet$ & $\bullet$ & & $\bullet$ & & & & 4 & 352.3 & 1.11 & 0.082 \\
\hline-0.1000 & $\bullet$ & $\bullet$ & $\bullet$ & $\bullet$ & $\bullet$ & $\bullet$ & & 7 & 352.7 & 1.49 & 0.067 \\
\hline-0.0610 & $\bullet$ & $\bullet$ & $\bullet$ & $\bullet$ & $\bullet$ & & $\bullet$ & 7 & 353.0 & 1.76 & 0.059 \\
\hline-0.2717 & $\bullet$ & • & & $\bullet$ & $\bullet$ & $\bullet$ & & 6 & 353.1 & 1.85 & 0.057 \\
\hline
\end{tabular}

Table 3: Overview of model selection statistics for models predicting search time of Cotesia glomerata. Explanatory variables included were accession (ac), distance from release plant (dst), marking of parasitoid (mrk), infestation of plant with Pieris brassicae caterpillars (trm) and two-way interactions between these variables. Selected variables are shown as a "•". Models are ranked based on the Akaike Information Criterion corrected for finite sample sizes (AICC). Only models with substantial support from the data are presented $(\triangle \mathrm{AICc} \leq 2)$.

\begin{tabular}{|c|c|c|c|c|c|c|c|c|c|c|c|}
\hline (int) & $a c$ & $d s t$ & $m r k$ & trm & $a c^{*} d s t$ & $a c^{*}$ trm & $d s t^{*}$ trm $a c^{*} d s t^{*}$ trm & $d f$ & $\mathrm{AICC}$ & $\triangle A I C C$ & weight \\
\hline 29.78 & & $\bullet$ & & $\bullet$ & & & & 4 & 982.7 & 0.00 & 0.122 \\
\hline 33.33 & $\bullet$ & $\bullet$ & & $\bullet$ & & $\bullet$ & & 6 & 983.0 & 0.26 & 0.107 \\
\hline 28.99 & & $\bullet$ & $\bullet$ & $\bullet$ & & & & 5 & 983.6 & 0.92 & 0.077 \\
\hline 31.00 & $\bullet$ & $\bullet$ & & $\bullet$ & & & & 5 & 983.7 & 1.00 & 0.074 \\
\hline 32.5 & $\bullet$ & $\bullet$ & $\bullet$ & $\bullet$ & & $\bullet$ & & 7 & 983.8 & 1.07 & 0.071 \\
\hline 34.90 & $\bullet$ & $\bullet$ & & $\bullet$ & $\bullet$ & $\bullet$ & & 7 & 983.8 & 1.09 & 0.071 \\
\hline 34.24 & $\bullet$ & $\bullet$ & $\bullet$ & $\bullet$ & $\bullet$ & $\bullet$ & & 8 & 984.0 & 1.30 & 0.063 \\
\hline 32.73 & $\bullet$ & $\bullet$ & & $\bullet$ & - & & & 6 & 984.4 & 1.66 & 0.053 \\
\hline
\end{tabular}




\section{Chapter 4}




\section{Plant traits interact with habitat context in determining the strength of host-parasitoid relationships}

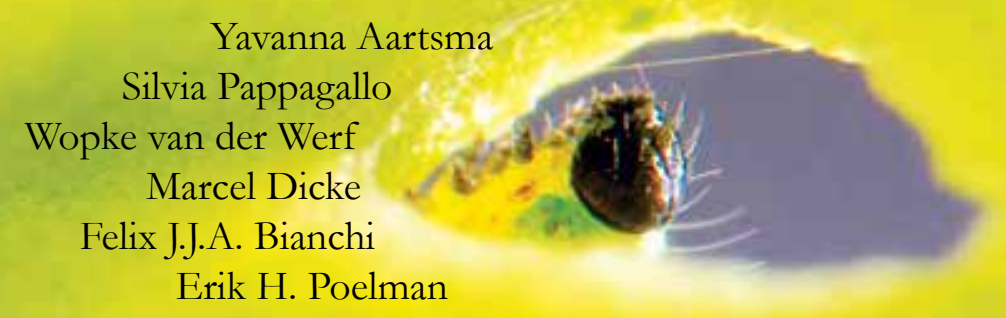




\section{Abstract}

Resources are often spread heterogeneously across habitats and, therefore, organisms need to forage for them. In tritrophic interactions between plants, herbivores and parasitoids, habitat context can affect foraging behaviour of herbivores and parasitoids. For insect herbivores, traits of the food plant can affect host-parasitoid interactions directly via plant quality or indirectly by altering parasitoid foraging behaviour by, for instance, the emission of herbivore-induced plant volatiles (HIPVs). At the same time, the habitat context surrounding a plant can alter behaviour of both herbivores and parasitoids, for example via patch size or the identity of neighbouring plant species. While the effects of focal plant traits and habitat context have been extensively studied in isolation, less is known on the interplay between focal plant traits and habitat context. We conducted a factorial field experiment with white cabbage (Brassica oleracea) accessions that differed in the attractiveness of the HIPV profile for parasitoids, plot size and presence of a Brassica nigra border. Throughout the growing season, parasitism rates of experimentally introduced Pieris brassicae caterpillars and the presence of naturally occurring Pieris spp. caterpillars in the plots were assessed. The abundance of the caterpillar Pieris rapae was neither affected by cabbage accession nor plot size. Later in the season, when B. nigra plants had senesced, fewer P. rapae caterpillars were found on cabbage plants in plots with a B. nigra border. Parasitism rates fluctuated over the season, and were not affected by plot size. However, the border negatively affected parasitism rates on the accession which is less attractive to the parasitoid Cotesa glomerata, but not on the more attractive accession. Superparasitism rates confirmed that the attractive accession sustained higher parasitism rates in the field overall. Our results show that traits of the focal plant (attractiveness to parasitoids) can lead to different outcomes on members of the second and third trophic level depending on characteristics of the surrounding plot context. These findings underscore the importance of considering the interaction between focal plant traits and habitat context for understanding parasitoid-host interactions. 


\section{Introduction}

Organisms need to find resources that are commonly spread heterogeneously across habitats that may differ in vegetation structure. Resources can be distributed in patches in a matrix of unsuitable habitat, thus effectively rendering these patches small islands (MacArthur \& Pianka, 1966). In tritrophic interactions between plants, herbivores and predators, foraging behaviour of herbivores and predators may be affected by characteristics of these patches, such as host plant identity, vegetation structure and patch size, which we will refer to as habitat context. In a metapopulation composed of patches of varying quality, apparency or vegetation structure, some patches may provide a refuge for herbivores, while others may lead to overexploitation by natural enemies (Van Nouhuys \& Hanski, 2002; van Nouhuys, 2005). Thus, variation in habitat context may determine local strength and stability of tritrophic interactions.

Plant-host-parasitoid systems are ubiquitous and well-studied for habitat context dependency of these tritrophic level interactions (Cronin \& Reeve, 2005; Forbes et al., 2018). Host-parasitoid dynamics are expected to be tightly linked, because parasitoid populations are strongly dependent on the abundance of their hosts at the patch scale (Hagen et al., 2012). When the host is a herbivore, these interactions can be influenced by traits of the herbivore's food plant (Kos et al., 2011). For example, host plant traits can affect herbivore immune responses against parasitoids (Bukovinszky et al., 2009; Smilanich et al., 2009) and plants differ in the strength and chemical composition of herbivore induced plant volatiles (HIPVs) that are used by parasitoids to locate their host (Vet \& Dicke, 1992; Beyaert \& Hilker, 2014; Turlings \& Erb, 2018). Because parasitoids may respond to different cues than herbivores, variation in these traits may affect these trophic levels differentially and cause spatial variation in the strength of trophic interactions. At the same time, aspects of the surroundings of the herbivore's food plant can also influence tritrophic interactions by altering foraging behaviour of herbivores and parasitoids (Wäschke et al., 2014; Moreira et al., 2016). Whereas the effect of habitat complexity and, to a lesser extent, plant traits on parasitoidhost interactions have received considerable attention, much less is known how the interplay between vegetation structure and food plant traits influences the 
strength and stability of parasitoid-host interactions. Most studies define trait variation a posteriori (Moreira et al., 2016), but do not investigate interactions between habitat structure and plant traits in a systematic way. Plant intraspecific variation in traits can lead to cascading effects on tritrophic interactions (Kos et al., 2011) and can alter food web structure (Bukovinszky et al., 2008).

The outcome of plant trait interactions with herbivores and parasitoids may depend on the plant community in which it is embedded. For example, neighbouring plants of different species can lead to a decrease or increase in herbivore damage, a phenomenon called associational resistance or susceptibility (Barbosa et al., 2009). Increasing vegetation complexity can result in lower foraging efficiency of parasitoids (Gols et al., 2005; Bukovinszky et al., 2007; Bezemer et al., 2010; Kruidhof et al., 2015). This effect could be attributed to neighbouring plants physically obstructing host plants or when volatiles emitted by these plants interfere with volatiles of the host plants. Neighbouring plants may mask a focal plant, or may specifically attract certain herbivores. Neighbouring plants may also interact with focal plant traits such as attractiveness via HIPVs. The surrounding vegetation can also provide structural challenges to foraging parasitoids by being physical barriers, or can limit the spread of HIPVs which are used as cues to find the host (Aartsma et al., 2017). However, there can be positive effects as well, for example by increasing contrast between background and focal plant patch (Soler et al., 2007) or by providing additional resources to the insect, such as nectar (Winkler et al., 2009; Rezende et al., 2014).

The number of plants in a patch or their density might also affect colonization by herbivores and parasitoids. For example, patch size may moderate the abundance of an insect species in the patch (Hambäck \& Englund, 2005). For small parasitoids with a low dispersal capacity, large monoculture patches might hamper their ability to enter the patches while at the same time keeping access to floral resources in other areas (Tscharntke et al., 2005b). Additionally, patch size may interact with the presence of a border of another plant species, because the ratio between plants inside the patch and surrounding plants changes. For example, if a weakly attractive herbivore-infested plant is weakly apparent to parasitoids, being in a larger patch of more weakly attractive plants might 
compensate for this, increasing the chance of attracting parasitoids.

In this study we investigated the outcome of parasitoid-host interactions for the tritrophic relationship of Brassica-Pieris-Cotesa glomerata in spatially heterogeneous environments. We focussed on the effects of plant genetic variation in HIPV production, patch size, and surrounding vegetation, including their interactions. Because we know from previous work that HIPVs from the cabbage accession Christmas Drumhead are more attractive to parasitoids than HIPVs from the accession Badger Shipper, we expected higher parasitism rates on the more attractive accession than on the less attractive accession (Poelman et al. 2009b; Aartsma et al. 2018). No differences in Pieris sp. abundance were expected due to plant accession (Poelman et al., 2009a). We expected Pieris caterpillars to be more abundant in small plots because these contrast with background vegetation (Bukovinszky et al., 2005), but to have lower risk of parasitism in these smaller plots because parasitoids seem to be more attracted to larger patches (Bezemer et al., 2010). To provide a surrounding vegetation, we added a border of Brassica nigra plants around cabbage patches. Brassica nigra is also a host plant to Pieris herbivores and, therefore, might also attract Pieris herbivores and C. glomerata. Therefore, we expected to find a positive effect of this plant species on parasitism rates and herbivore abundance in cabbage plots surrounded by a border. Finally, we discuss how plant traits and habitat complexity contribute to host-parasitoid interactions.

\section{Materials and Methods}

\section{Plants and insects}

White cabbage (Brassica oleracea var alba) accessions Badger Shipper and Christmas Drumhead were used in the experiment. Seeds were obtained from the Centre for Genetic Resources (CGN-Wageningen, the Netherlands). For the border surrounding some of the plots, Brassica nigra was used. Brassica nigra seeds originated from a field population near Wageningen, The Netherlands, and were multiplied by open pollination for two generations. All plants were grown in peat soil in a greenhouse at Unifarm, Wageningen (L16:D8, $18-26{ }^{\circ} \mathrm{C}$ and $40-70 \%$ RH) and were transplanted to the field in May 2015 as 4 week old seedlings. 
Pieris brassicae caterpillars were reared on Brussels sprouts plants in a greenhouse compartment $\left(20-22{ }^{\circ} \mathrm{C}\right.$ and $\left.50-70 \% \mathrm{RH}\right)$ and L1 and L2 caterpillars were used in the experiment.

\section{Experimental design}

Field plots were established according to a $2 \times 2 \times 2$ factorial design in May 2015. Plot size was small or large $(3 \times 3$ or $9 \times 9$ cabbage plants, respectively), with or without a border of B. nigra plants, and plots contained either the less attractive accession Badger Shipper or the more attractive Christmas Drumhead. Thus, there were eight treatments, which were each replicated seven times (Fig. 1). Plants within a plot were spaced $70 \mathrm{~cm}$ apart, and there was at least $6 \mathrm{~m}$ between the plots. Two rows of white cabbage plants (variety Lennox) were planted around the full field experiment $(92 \times 96 \mathrm{~m})$ to minimize border effects. In the days after transplanting the seedlings, the seedlings suffered from extensive damage by wood pigeons. Over the next few weeks, all plots fully recovered except for one small Christmas Drumhead plot with border. This treatment thus had only six replicates.

\section{Field observations}

The abundance of herbivorous hosts of C. glomerata, caterpillars of Pieris brassicae and Pieris rapae that colonized the plots were recorded in each plot in mid-season (weeks 29 and 30) and late season (weeks 32 and 33). In plots with $3 \times 3$ cabbage plants, four Brassica oleracea were monitored, and in plots with $9 \times 9$ cabbage plants, eight cabbage plants were monitored (Fig. 1B). The central plant of the plot, which was used for assessing parasitism rates, was not used to monitor herbivore abundance. In plots with a border, four and eight Brassica nigra plants from the border were also monitored in small and large plots, respectively (Fig. 1B).

Parasitism was assessed on a weekly basis by placing 10 first instar (L1) P. brassicae caterpillars on the centre plant of each plot. After four days, the caterpillars were recollected and dissected under a stereomicroscope to assess the number of $C$. glomerata eggs in each caterpillar. This procedure was repeated during eight weeks from June until August 2015 (weeks 24 to 32, with the exception of week 27). The experiment was terminated in week 33 , because the cabbage plants were 
either senescent or the leaves were too tough for the first instar caterpillars to feed on.

\section{Statistical analysis}

Herbivores

Naturally occurring P. brassicae caterpillars were encountered on only 4 and 13 plants out of the 496 plants monitored during mid- and late season, respectively, and their abundance was low $(0.0068 \pm 0.0039$ and $0.28 \pm 0.21$ caterpillars/ plant/plot, respectively). This did not allow a meaningful comparison between treatments and these data were, therefore, not statistically analysed.

A

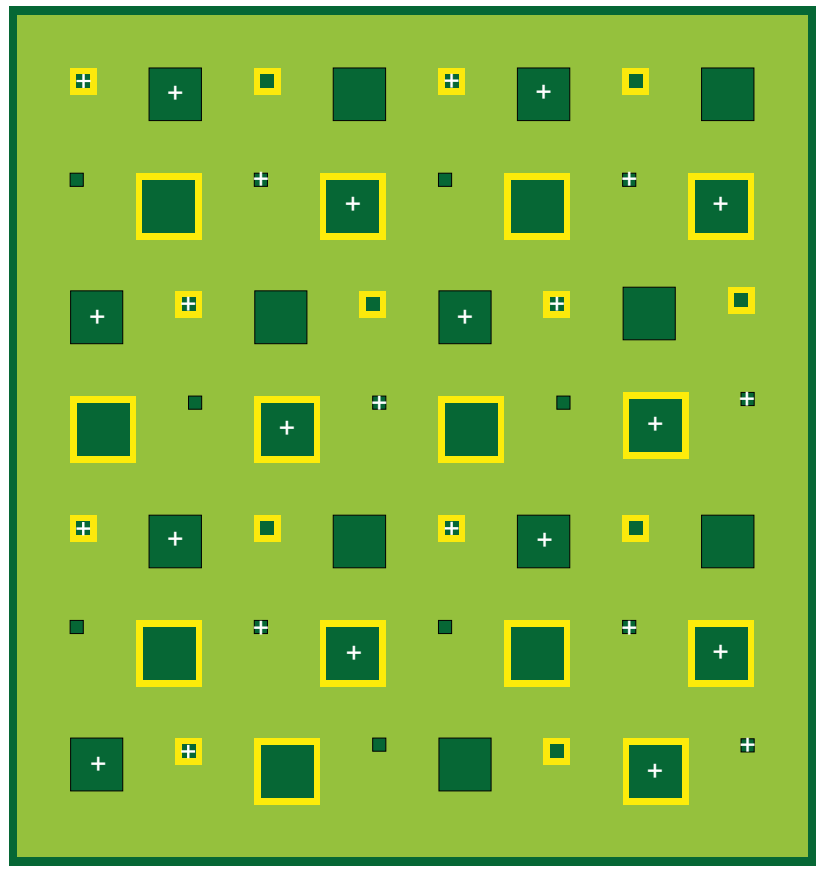

B

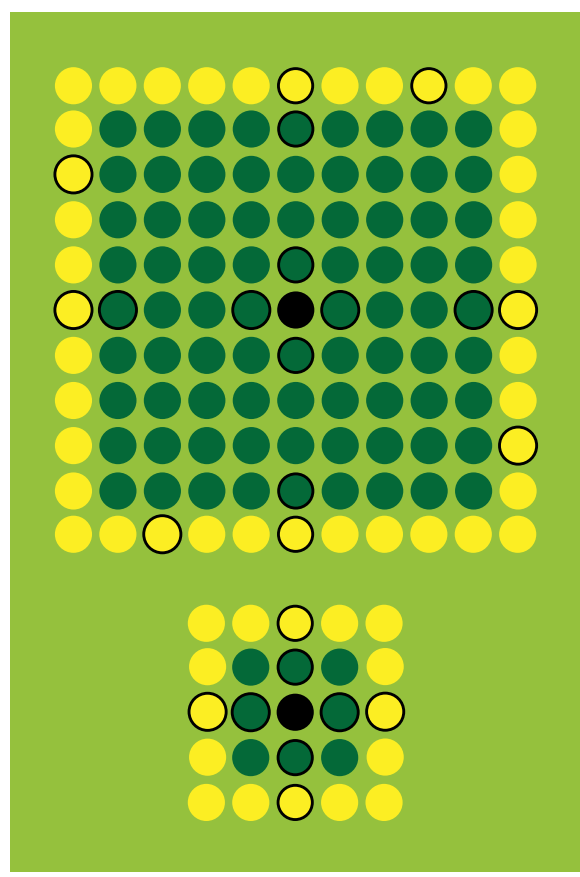

Figure 1: A: Experimental set-up. Large and small squares indicate plots of $9 \times 9$ and $3 \times 3$ cabbage plants, respectively. Christmas Drumhead plots are indicated with "+" and plots without marker contained Badger Shipper. Plots with Brassica nigra border are indicated with a yellow border, which was planted at the same time as the cabbage. Grass was sown between the plots and the field was surrounded by a border of white cabbage of the accession 'Lennox'. B: Example of a large and small plot with border. Cabbage plants and $B$. nigra plants are indicated by green and yellow circles, respectively. Plants used for herbivore monitoring are indicated with a black outline. The plant used for experimental inoculation with P. brassicae caterpillars to assess parasitism rates is indicated as a closed black circle. 
To investigate whether abundance of naturally occurring $P$. rapae caterpillars differed between plots with different accessions, sizes, and border and at different sampling times, we used a generalized linear model with negative binomial error distribution and $\log$ link. The response variable was the number of $P$. rapae caterpillars on monitored plants, and the number of monitored plants per plot was used as an offset variable with a log transformation. Accession, border, plot size, and their two-way interactions were included as fixed factors. We used a model selection procedure using the 'dredge' package in $\mathrm{R}$, which calculates all models with all fixed factor combinations and sorts them based on the Akaike Information Criterion corrected for small sample sizes (AICc).

Pieris rapae abundance was analysed in more detail by considering three different response variables in separate models: (i) $P$. rapae on cabbage plants only, (ii) $P$. rapae on B. nigra border plants only, and (iii) P. rapae on cabbage and B. nigra border plants combined. For all three response variables we used the same model as described above with adjusted number of monitored plants as offset variable. In the late-season monitoring period, B. nigra plants in the borders were senescing and only a single $P$. rapae caterpillar was found in the borders. Therefore, for lateseason only the count data for $P$. rapae on cabbage was used.

\section{Parasitism rate}

Generalized linear mixed models with binomial error distribution were used to investigate whether parasitism rates (response variable) were affected by accession, plot size, border presence, time of the year (fixed factors) and their interactions. We included an observation level random effect to account for overdispersion in the data. We used the same model selection procedure as described above for the herbivores. The interaction between factors was analysed in more detail with a Tukey post-hoc test.

\section{Superparasitism}

Cotesa glomerata females rarely deposit more than 35 eggs per caterpillar (Poelman et al., 2013). Therefore, we scored caterpillars with more than 35 eggs as being superparasitized. To investigate whether superparasitism rates were affected by accession, plot size, border presence and time of the year, we used a generalized linear mixed model with a binary response variable "superparasitism" (i.e 
presence or absence). Accession, plot size, border presence and week were included as fixed factors, and two-way interactions were included as well. Plot ID was included as random factor. We used the 'dredge' procedure to identify the most parsimonious models. Data were analysed in $\mathrm{R}$ using the packages lme4, MuMIn, MASS, multcomp and ggplot.

\section{Results}

\section{Herbivores}

The average abundance of P. rapae was $0.29 \pm 0.044$ and $0.38 \pm 0.054$ caterpillars/ plant/plot (mean $\pm S E M$ ) for cabbage (Christmas Drumhead and Badger Shipper) and B. nigra plants combined in mid- and late season, respectively. In the midseason monitoring period, P. rapae caterpillars were present both on cabbage plants

Table 1: Determinants of Pieris rapae abundance on Brassica oleracea and/or Brassica nigra plants in plots with different size ( $3 \times 3$ plants or $9 \times 9$ plants), with or without a B. nigra border, and with the attractive Brassica oleracea accession (Christmas Drumhead) or less attractive accession (Badger Shipper) in a generalized linear model with negative binomial error distribution and log link. The factor 'border' was not included for $P$. rapae abundance on $B$. nigra, as this plant species was only present in plots including a border. In the late season, $P$. rapae was not present on $B$. nigra border plants and therefore only $P$. rapae abundance on the cabbage ( $B$. oleracea) plants was analysed. Estimates with standard error (between brackets) and statistical significance (bold and with asterisks) are reported. Only main effects are shown, as interactions were not significant. Reference treatments are "9 × 9 plants", "no border" and "Badger Shipper".

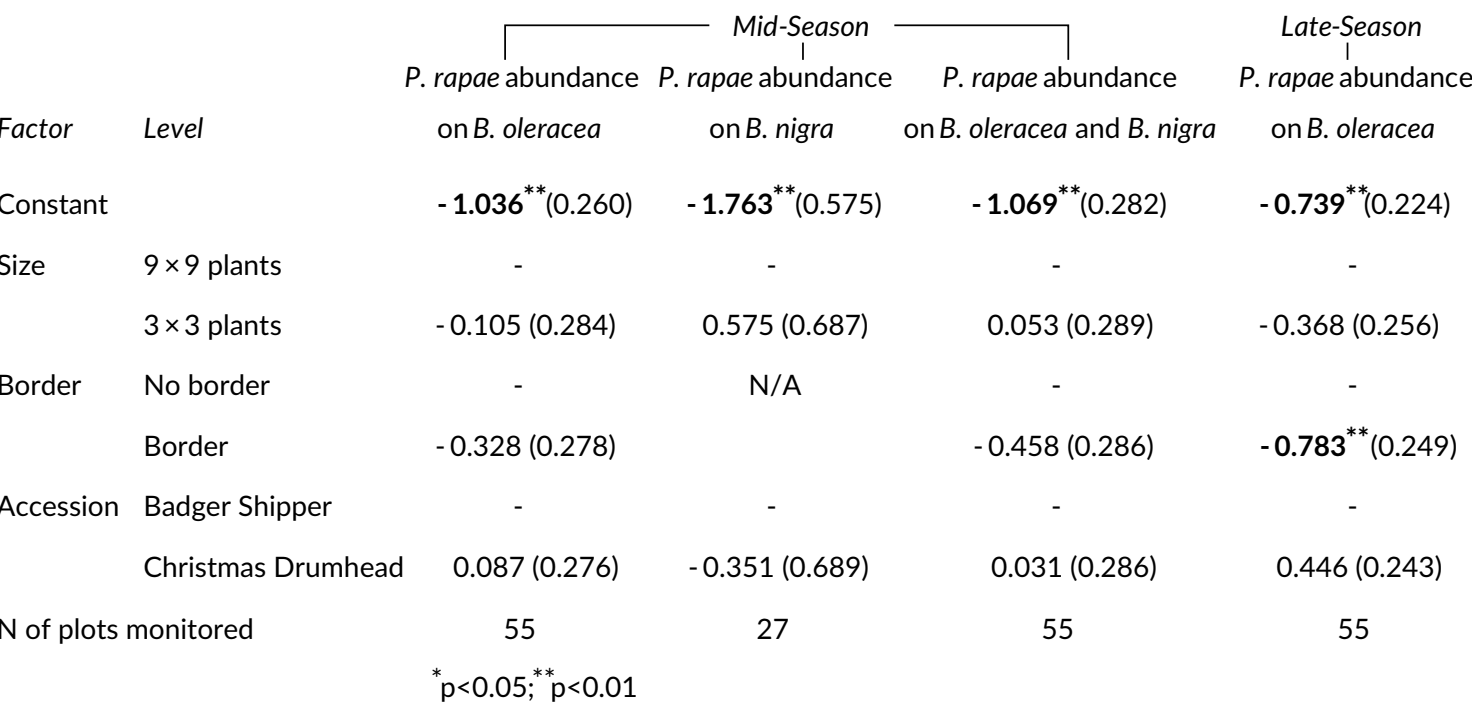


within the plots and on B. nigra plants in plots with a border. Pieris rapae abundance on cabbage, $B$. nigra or combined was not significantly related to plot size, presence of a border or cabbage variety (Table 1 ). In the late season, $P$. rapae abundance on cabbage plants was lower in plots surrounded with a B. nigra border than plots without border, but was not significantly different between large and small plots or between accessions Badger Shipper and Christmas Drumhead (Table 1).

Table 2: plants in plots with different size $(3 \times 3$ plants or $9 \times 9$ plants), with or without a Brassica nigra border, and with the attractive Brassica oleracea accession (Christmas Drumhead) or less attractive accession (Badger Shipper) and the different weeks of the experiment in a generalized linear mixed model with binomial error distribution and log link. No border, large size, the less attractive accession Badger Shipper and week 24 were the reference treatments for border, size, accession and week, respectively. Plant ID was used in the superparasitism analysis as a random effect to account for caterpillars cooccurring on the same plant at the same time. A slash symbol (/) indicates that this variable was not selected in this model. Estimates are shown, with standard error between brackets and statistical significance in bold and with asterisks *

\begin{tabular}{|c|c|c|c|}
\hline Factor & Level & Parasitism (parasitized/total) & Parasitism (0) or su \\
\hline Constant & & $2.517^{* * *}(0.670)$ & $-2.386^{* * *}(0.377)$ \\
\hline \multirow[t]{2}{*}{ Border } & No border & - & / \\
\hline & Border & $-1.621^{* *}(0.589)$ & \\
\hline \multirow[t]{2}{*}{ Accession } & Badger Shipper & - & - \\
\hline & Christmas Drumhead & $0.547(0.605)$ & $0.946^{* * *}(0.269)$ \\
\hline \multirow[t]{2}{*}{ Size } & $9 \times 9$ plants & - & / \\
\hline & $3 \times 3$ plants & $0.680(0.436)$ & \\
\hline Border $\times$ accession & - & $1.879^{*}(0.873)$ & / \\
\hline \multirow[t]{8}{*}{ Time } & Week 24 & - & - \\
\hline & Week 25 & $-4.394^{* * *}(0.795)$ & $-2.547^{* * *}(0.757)$ \\
\hline & Week 26 & $-2.741^{* * *}(0.769)$ & $0.609(0.544)$ \\
\hline & Week 28 & $2.320^{*}(0.911)$ & $4.757^{* * *}(0.618)$ \\
\hline & Week 29 & $3.096^{* * *}(0.867)$ & $1.711^{* * *}(0.434)$ \\
\hline & Week 30 & $0.172(0.801)$ & $-0.718(0.486)$ \\
\hline & Week 31 & $0.607(0.743)$ & $-0.786(0.466)$ \\
\hline & Week 32 & $3.295^{* *}(1.178)$ & $2.056^{* * *}(0.471)$ \\
\hline \multirow[t]{2}{*}{ Observations } & & 332 & 1371 \\
\hline & & ${ }^{*} p<0.05 ;{ }^{* *} p<0.01 ; \stackrel{* * *}{p}<0.001$ & \\
\hline
\end{tabular}




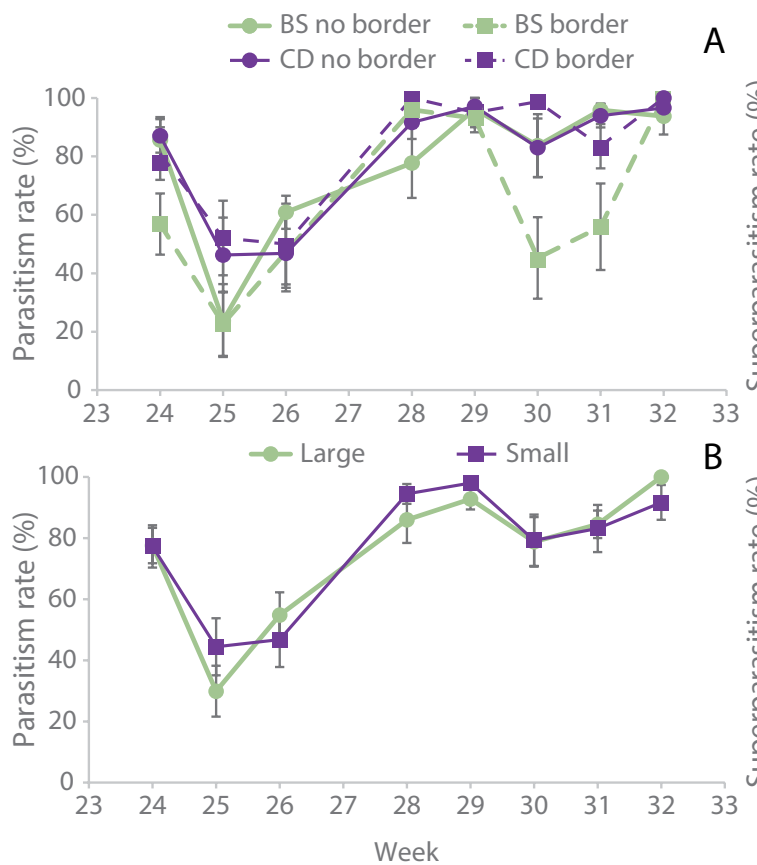

A
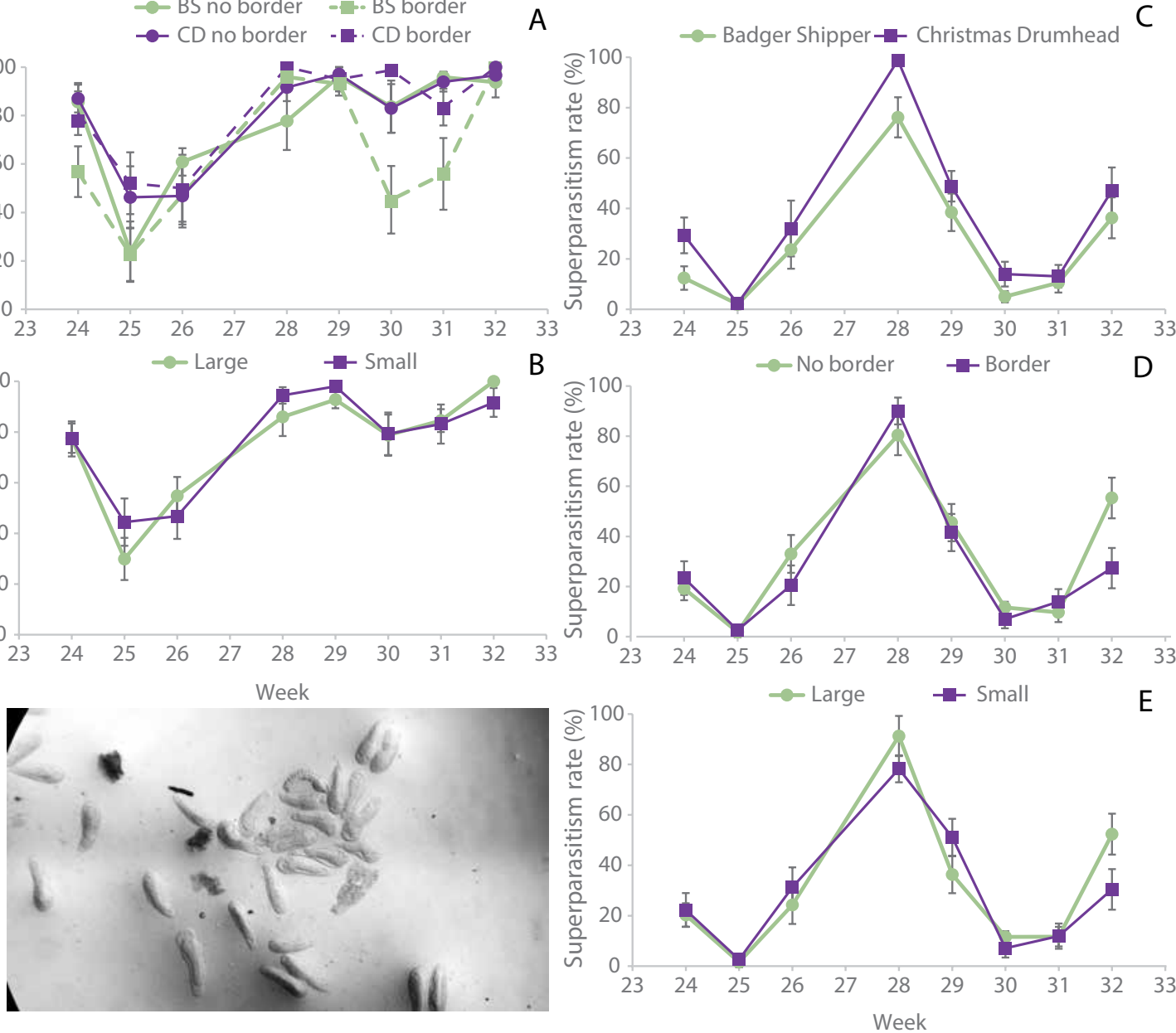

Figure 2: Parasitism rates (left) and superparasitism rates (right) of experimentally released Pieris brassicae caterpillars on the central Brassica oleracea plant of plots. Parasitism rates are calculated as the percentage of caterpillars parasitized out of the P. brassicae caterpillars recovered from a plot. Caterpillars with more than 35 eggs were considered to have been superparasitized, and superparasitism was calculated as the percentage of parasitized caterpillars that were superparasitized. Panel A shows the interaction between accession (less parasitoid-attractive accession Badger Shipper (BS) or more parasitoid-attractive accession Christmas Drumhead (CD)) and Brassica nigra border (absent or present). Panels B and E display differences between plot sizes $(9 \times 9$ plants vs. $3 \times 3$ plants). Panel $C$ displays differences between Badger Shipper and Christmas Drumhead. Panel D displays differences between border absence or presence. Photo shows eggs of Cotesa glomerata after dissection of the caterpillars. 


\section{Parasitoids}

The overall recovery rate of the experimentally introduced caterpillars on the centre plants in plots was on average 39.6\%. Parasitism rates of these caterpillars varied over the season and approached $100 \%$ parasitism later in the season (Table 2, Fig. 2A-B). There was no significant effect of plot size on the parasitism of $P$. brassicae caterpillars on cabbage plants (Table 2). However, there was a significant interaction between cabbage variety and border presence, indicating that plots consisting of the less attractive variety Badger Shipper had lower parasitism rates when a border of $B$. nigra was present, while the presence of a border did not affect parasitism levels for plots of the more attractive variety Christmas Drumhead (Table 2, Fig. 2A, Fig. 3).

Superparasitism incidence fluctuated throughout the season (Table 2) and followed the temporal fluctuations of overall parasitism rates (Fig. 2). Overall, superparasitism was observed more frequently on the attractive accession

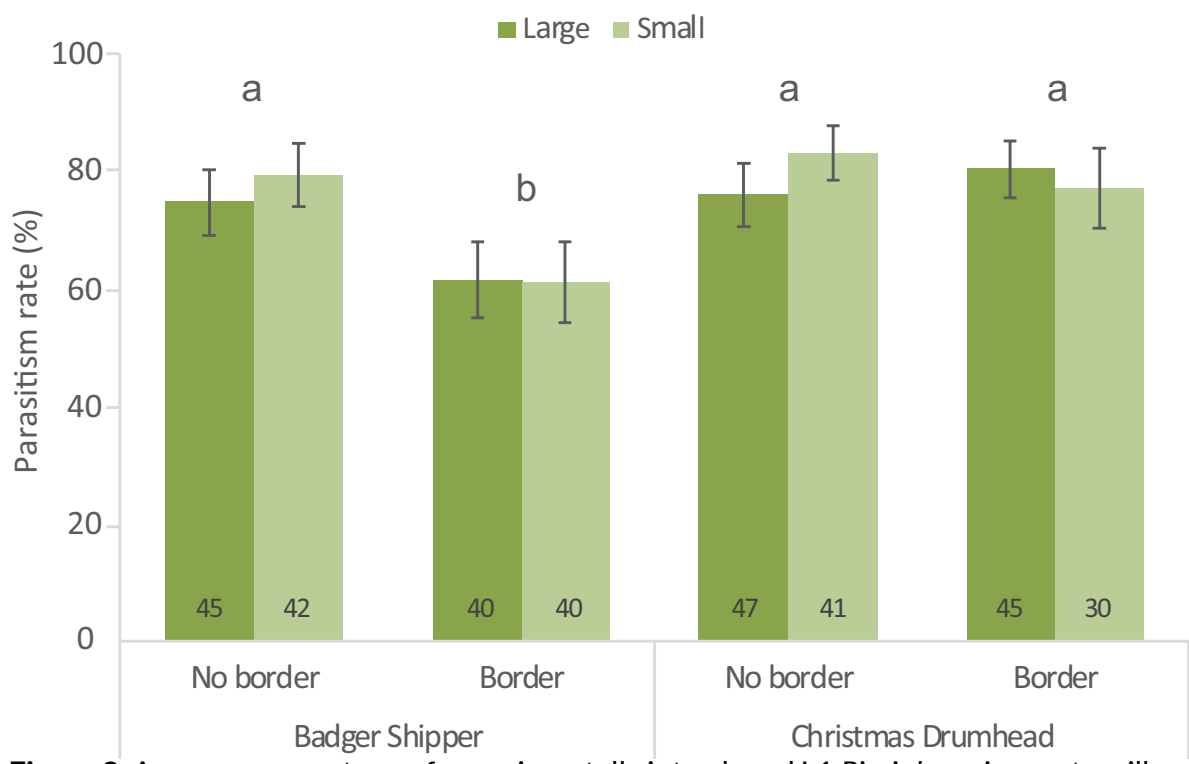

Figure 3: Average percentage of experimentally introduced L1 Pieris brassicae caterpillars that were parasitized on the central plant of plots differing in cabbage variety (Badger Shipper or Christmas Drumhead), plot size (large, $9 \times 9$ plants, or small, $3 \times 3$ plants) and presence of a border of Brassica nigra plants across 8 experimental weeks. Different letters indicate significant differences between combinations of border and cabbage variety (Tukey HSD). Error bars represent standard error of the mean and the number of observations is shown at the base of the bars. 
Christmas Drumhead than on accession Badger Shipper (Table 4, Fig. 2C), but was not affected by plot size and presence of a border (Table 4, Fig. 2D-E).

\section{Discussion}

While insect herbivores and their parasitoids are commonly confronted with heterogeneous environments, few studies have investigated how aspects of habitat structure interacts with intraspecific variation in focal plant traits to moderate parasitoid-host interactions. The results of this study highlight the differential responses of herbivores and their primary parasitoids to habitat complexity, and point to the complex interaction between plant traits and vegetation structure for host finding by parasitoids, and the associated strength of tritrophic interactions. Our study has three key findings. First, herbivore infestation was lower in plots surrounded by borders at the end of the season, but not in the middle of the season. Second, a B. nigra border was associated with lower parasitism rates, but only on the less attractive accession Badger Shipper. Third, superparasitism was consistently highest in plots with the attractive accession Christmas Drumhead.

\section{Herbivores}

There was no significant difference in P. rapae abundance between plots with the two cabbage varieties at any of the two assessments during the growing season, which aligns with results of a previous field study using the same accessions (Poelman et al., 2009a). Apparently, P. rapae does not differentiate between the plant volatiles of the two cabbage accessions. However, our finding that plot size did not influence $P$. rapae abundance contrasts with an earlier field study by Bukovinszky et al. (2005), which compared similar patch sizes as we did here. More oviposition by $P$. rapae was found in smaller cabbage plots, regardless of the vegetation type surrounding these plots (Bukovinszky et al., 2005). In a simulation study, it was postulated that $P$. rapae uses visual cues to find cabbage patches and that small patches are more conspicuous in the background vegetation (Bukovinszky et al., 2005; Hambäck \& Englund, 2005). A visual search mode in combination with a high movement capacity might explain why P. rapae abundance was not higher in large plots. However, our study does not 
confirm that smaller plots are associated with a higher abundance of $P$. rapae than large plots. This may be explained by the fact that the $P$. rapae abundance in our study was generally lower than in the study by Poelman et al. (2009a) with the same accessions, which makes it harder to demonstrate significant differences between treatments.

In the late season, there were fewer P. rapae caterpillars in plots with a $B$. nigra border. At this point in time, the B. nigra plants had completed their development and were senescent, suggesting that the age of the border plants may have influenced oviposition preference of the butterflies. Since P. rapae butterflies use a visual searching mode (Bukovinszky et al., 2005), this suggests that the border visually obstructed perception of suitable host plants inside the plot. While visual cues such as leaf colour have been found to decrease colonization of plants with neighbouring plants (Finch et al., 2003), we cannot exclude that the B. nigra plants may have interfered with volatile cues from the cabbage as well.

\section{Parasitoids}

Parasitism by Cotesia spp. was lower on the less attractive cabbage variety Badger Shipper when surrounded by a border of B. nigra plants as compared to plots without this border. In contrast, parasitism rates on the more attractive cabbage variety Christmas Drumhead were not affected by the presence of a border. While the border could provide both a physical and visual barrier for parasitoids, and potentially act as a barrier for odour plumes (Aartsma et al., 2017), our data suggest that the border may hamper the perception of HIPVs from the less attractive accession, but not of the attractive accession. Thus, a more attractive variety may be more chemically apparent for parasitoids in patches with a complex vegetation structure. Previously, we found that the more attractive accession is able to attract parasitoids from a greater distance than the less attractive accession (Aartsma et al., 2018). The relative attractiveness of $B$. nigra plants as compared to Badger Shipper and Christmas Drumhead is not known and merits further investigation.

In the absence of a border, parasitism rates were not significantly different in plots with attractive and less attractive accessions. However, the absence of an accession effect 
could have been masked by the high parasitism rates in the experiment, particularly later in the season, when parasitism rates approached 100\% (Fig. 2). Interestingly, superparasitism incidence was indeed higher on the more attractive variety than on the less attractive variety, confirming higher parasitoid visitation in plots with the attractive accession (Poelman et al., 2013; Aartsma et al., 2018). Thus, the assessment of superparasitism allowed us to demonstrate the higher visitation rates in plots with the attractive accession, despite the saturation of parasitism in plots.

Parasitism rates were not affected by plot size. Therefore, our hypothesis that larger plots might attract more parasitoids by generating a larger amount of HIPVs was not confirmed by our data. There was also no significant interaction of plot size with the attractiveness of the plant accession used in the plots. In the experiment, we only experimentally inoculated the central plant of the plot with caterpillars. As the abundance of Pieris caterpillars from natural infestations was relatively low, the other plants in the plot may not have been induced to produce HIPV-blends attractive to C. glomerata, and therefore played a minor role in attracting this species to the central plants. Further studies may reveal whether the effect of plot size is more pronounced in years with higher natural infestations of Pieris butterflies.

\section{Response of herbivores vs response of parasitoids}

In contrast to the herbivores, the parasitoids were affected by cabbage accession and border type. The difference in responses by members of these two trophic levels may be mediated by the type of cues they use (HIPVs vs general plant odours). The spatial distribution of odour cues can be affected by structural and chemical barriers from surrounding vegetation, resulting in a spatially heterogeneous volatile mosaic (Randlkofer et al., 2010b; Aartsma et al., 2017). In addition, background odours can play a role in masking of chemical cues (Vos et al., 2006; Hilker \& McNeil, 2008; Schröder \& Hilker, 2008). A weak emitter of cues can be 'hidden' in background odours from neighbouring plants (Vos et al., 2006). A stronger emitter of these cues (i.e. a more attractive variety) might be easier to distinguish from background odours emitted by other plants. It should be noted, however, that attractiveness or apparency of host infested plants can be due to both quantitative and qualitative aspects of HIPVs (Clavijo McCormick et al., 2012; Rowen \& Kaplan, 2016), which are difficult to disentangle under field conditions. 
Besides the chemical composition of plant-mediated volatile cues, the dispersal capacity of insects might also affect how different trophic levels are impacted by habitat context. There are several studies that show that herbivores tend to have a higher dispersal capacity than their parasitoids, which may be related to differences in body size (Van Nouhuys \& Hanski, 2002; Tscharntke \& Brandl, 2004; van Nouhuys, 2005). Therefore, herbivores may generally operate at larger scales than their parasitoids, and may be less affected by barriers, such as the B. nigra border in our study. The contrasting responses of herbivores vs parasitoids can be important for host-parasitoid interactions. Herbivores may escape parasitism by colonizing areas that are more difficult to find/reach by the less mobile parasitoids. Habitat heterogeneity can play are role in providing herbivores with refuges (Gols et al., 2005; Bukovinszky et al., 2007; Karban et al., 2013). Herbivores may use sub-optimal plants to escape from parasitoids (Ohsaki \& Sato, 1994) or use habitat types less suitable for their parasitoids (Ohsaki \& Sato, 1999).

In conclusion, this study shows that both habitat structure and variation in focal plant traits such as HIPV emission can affect tritrophic interactions. Thus, variation in habitat context can have different effects depending on traits of the focal plant, and plants with variation in traits can also differ depending on the vegetation context in which they are embedded. Since there are few other studies that focussed on the interaction between variables related to habitat structure and plant traits such as attractiveness to parasitoids in a systematic way, it is not clear how general our findings are. There is scope for further research on the way how vegetation structure and plant traits associated with the communications between trophic levels interact in different systems and conditions. 
Plant traits interact with habitat context 


\section{Chapter 5}




\section{Herbivory by hosts and non-hosts, in interaction with plant traits, affects herbivore-induced plant volatiles and foraging efficiency of a specialist parasitoid}

\section{Yavanna Aartsma}

Miguél Calvo Agudo

Cassandra Vogel

Alexandre Villela

Desalegn W. Etalo

Wopke van der Werf

Marcel Dicke

Felix J.J.A. Bianchi

Erik H. Poelman 


\begin{abstract}
Trophic interactions often occur in a species-rich community context. To locate their herbivore host in these communities, parasitoids exploit herbivore-induced plant volatiles, but such volatiles may be induced by both hosts and non-hosts. Furthermore, different plant genotypes emit different HIPV profiles and they respond differently to feeding by specific herbivores. Little knowledge is available about how efficiently foraging parasitoids can locate hosts using HIPV cues when they are confronted with a blend of HIPV cues resulting from feeding by hosts and non-hosts on plant genotypes of varying attractiveness. Here, we investigated how variation in plant attractiveness and distribution of host and non-host herbivores over these plants affect host-finding success of a parasitoid wasp in a field-tent experiment. We further studied whether differences in hostfinding success can be explained by HIPVs in a wind tunnel and analysed volatile blends emitted by plants of the same or different genotypes that were infested by hosts, non-hosts, or both (dual infestation). Our results show that dual infestation of a less attractive plant accession results in reduced host-finding success when it is present near a more attractive plant infested by only hosts; in contrast, dual infestation on both plant accessions did not reduce host-finding success. HIPV blends differed between the two accessions, but these differences did not fully explain parasitoid host-finding success in complex situations. We discuss our findings in the context of HIPV-mediated foraging behaviour and patch foraging processes and conclude that plant genotypic variation in HIPVs and the presence of non-host herbivores intricately interact in their effect on parasitoid host finding.
\end{abstract}




\section{Introduction}

Interactions between two species may be influenced by the presence of other species. These indirect species interactions are particularly apparent in predatorprey interactions, for example in prey switching by predators, intra-guild predation and apparent competition (Abrams \& Matsuda, 1996; Werner \& Peacor, 2003; Arim \& Marquet, 2004). One mechanism by which interactions between species can be influenced is by emission of chemical cues that predators and parasitoids use to locate prey and hosts (van Alphen et al., 2003; Vos et al., 2006). In both terrestrial and aquatic ecosystems, chemical cues have been well-studied (Raguso et al., 2015; Kamio \& Derby, 2017) and they are affected by the presence of other species in the community (Vos et al., 2001; Utsumi et al., 2010; Poelman, 2015). In species-rich plant-insect communities, indirect interactions are strongly affected by two sources of variation. On individual plants, multiple herbivore species may alter chemical cues released by the plant and this affects foraging by herbivores or predators (de Rijk et al., 2013; Stam et al., 2014). Additionally, plants are often embedded in a complex vegetation structure where plant genotypic variation and the presence of other plant species can alter foraging behaviour of both herbivores and their natural enemies through alteration of chemical cues (Randlkofer et al., 2010; Wäschke et al., 2014; Aartsma et al., 2017).

Plants under attack by herbivores respond by emitting herbivore-induced plant volatiles (HIPVs). Blends of HIPVs may differ between plant species or genotypes within a species and provide specific information to herbivores and predators regarding the species and quality of the plant, as well as presence of different herbivore species (Rasmann et al., 2005; Poelman et al., 2009b; Mumm \& Dicke, 2010; Gols et al., 2011). Predators and parasitoids can be more attracted to certain plant varieties, which can result in higher predation and parasitism rates on these plants in the field (Poelman et al., 2009b). Multispecies herbivore assemblages that comprise suitable prey/hosts as well as non-prey or non-host species can alter the information presented to predators or parasitoids (de Rijk et al., 2013). For instance, non-host herbivores can reduce parasitoid foraging efficiency by making it more difficult to discriminate between host-infested plants and plants not infested with hosts (Shiojiri et al., 2000; Bukovinszky et al., 
2012; Peñaflor et al., 2017). At the same time, dual herbivory (i.e. infestation by both hosts and non-hosts) often results in increased attraction of parasitoids (Shiojiri et al., 2000; Bukovinszky et al., 2012; de Rijk et al., 2016a), although feeding by exotic non-host species may reduce the attraction of parasitoids to dual-infested plants compared to plants infested with hosts only (Chabaane $e t$ al., 2015; Desurmont et al., 2018). Whether parasitoids are able to discriminate between host- and non-host infested plants may also depend on the plant species the herbivores feed on (Gols et al., 2012). However, so far it is unknown whether differences in HIPV blends as a result of plant species or genotypes interact with differences in induction by different herbivore species and the consequences for host location by parasitoids.

Specific knowledge gaps are: How do plant species or genotypes differ in their HIPV response to the combination of host and non-host herbivory, and what are the implications for the attractiveness to parasitoids? If the host is on a plant that emits a less attractive HIPV blend, but the non-host is on a plant that emits a more attractive HIPV blend, does this result in parasitoids visiting the nonhost infested plant more often than the host-infested plant? Or is the volatile blend emitted by non-host infested plants sufficiently different for parasitoids to still find the less attractive plant which actually is infested by hosts?

To address these issues, we considered a study system comprising host and nonhost herbivore species, and plants that differ in HIPV-mediated attractiveness to parasitoids. We investigated how variation in plant and herbivore identity affects host-finding success and behaviour of a parasitoid wasp. Two white cabbage accessions, which differ in HIPV-mediated attractiveness to the parasitoid Cotesia glomerata, were used in combination with infestation by the host of $C$. glomerata, Pieris brassicae caterpillars, and a non-host species, Plutella xylostella caterpillars. We studied the ability of the parasitoid to locate hosts in a field tent experiment and wind tunnel experiment. In addition, volatiles of the two accessions infested with hosts, non-hosts or both were collected to examine whether HIPVs could explain the foraging behaviour of C. glomerata. The plant accession Christmas Drumhead is a more attractive accession via HIPVs for the parasitoid Cotesia glomerata than Badger Shipper (Poelman et al., 2009b; Aartsma 
et al., 2018), and addition of the chewing non-host herbivore Plutella xylostella to host-infested plants may lead to increased preference for dual infested plants (Shiojiri et al., 2000). We expected that parasitoids have reduced host location efficiency when their host is feeding on an attractive plant accession and the less attractive accession is infested with a combination of host and non-host herbivores that by quantitatively more damage may attract part of the parasitoids to dual herbivore infested plants. Furthermore, we predicted that parasitoids are hampered in host location when their host is feeding on an unattractive plant accession while a more attractive accession is infested with non-host herbivores. We further expected that foraging efficiency in complex situations would be limited by interference between host and non-host induced HIPVs and that HIPVs induced by host and non-host herbivores differed for the two accessions.

\section{Materials and methods}

\section{Plants and insects}

We used two different accessions of white cabbage (Brassica oleracea var. alba L.), 'Badger Shipper' and 'Christmas Drumhead', in the experiments. Seeds of these accessions were obtained from the Centre for Genetic Resources (CGN Wageningen, The Netherlands). Upon feeding damage by the host caterpillar Pieris brassicae, both accessions are more attractive to the parasitoid Cotesia glomerata than undamaged plants of the same accession (Poelman et al., 2009b). Moreover, HIPVs emitted by Christmas Drumhead plants are more attractive to the parasitoid C. glomerata than HIPVs emitted by Badger Shipper (Poelman et al., 2009b). Plants were grown in $0.7 \mathrm{~L}$ pots filled with peat soil (Lentse potgrond, $\mathrm{n}^{\mathrm{o}} 4$, Lent, The Netherlands) in a greenhouse compartment $\left(22 \pm 4{ }^{\circ} \mathrm{C}, 16 \mathrm{~L}\right.$ : $8 \mathrm{D}, 40-70 \% \mathrm{RH})$. Plants used in the wind tunnel behaviour experiment and for collection of VOCs were 5 to 6 weeks old. In the field-tent experiments they were 7 to 8 weeks old.

Pieris brassicae and Plutella xylostella were reared under greenhouse conditions (20$22{ }^{\circ} \mathrm{C}, 16 \mathrm{~L}$ : 8D, and 50-70\% RH) on Brussels sprouts plants (Brassica oleracea var. gemmifera Cyrus). In all experiments first instar P. brassicae and second instar $P$. 
xylostella caterpillars were used because these larval stages are similar in size. The parasitoid C. glomerata was reared under the same greenhouse conditions, using P. brassicae as host insect. Cocoons and emerged adults of $C$. glomerata were kept in a Bugdorm cage $(30 \times 30 \times 30 \mathrm{~cm})$ in a climate cabinet $\left(20-22{ }^{\circ} \mathrm{C}\right)$ and had access to water and honey. Mated 5 -to-7-days old naïve female parasitoids were used in the experiments.

\section{Field-tent experiment}

We assessed host-finding success of $C$. glomerata in environments with two plant varieties differing in attractiveness and in the distribution of host and non-host herbivores over the two plant varieties in a field-tent experiment. The response variable of interest in this experiment was the number of hosts parasitized and number of plants with parasitized hosts, both as a total for the whole tent (not distinguishing between accessions) and comparing the two accessions within each tent. Twelve mesh tents of $3 \times 4 \times 2 \mathrm{~m}(\mathrm{~L} \times \mathrm{W} \times \mathrm{H})$ were set up in a mown monoculture field of perennial rye grass in Wageningen, The Netherlands, a setup similar to the one described in Bukovinszky et al. (2012). Grass was removed and cabbage plants were planted in bare soil in a $4 \times 4$ grid in each tent with a $0.5 \mathrm{~m}$ spacing between plants. In each tent, eight plants of accession Christmas Drumhead and eight plants of accession Badger Shipper were arranged in a checkerboard pattern (Fig. 1).

One week after planting, we infested the plants with host (P. brassicae) and non-host caterpillars (P. xylostella) according to four treatments (Fig. 1). First, to investigate the effect of plant accession on parasitoid foraging efficiency in a control treatment, plants of both accessions were infested with 10 host caterpillars (host treatment, Fig. 1A). Second, to examine the effects of addition of non-host herbivores, plants of both accessions were infested with 10 hosts and 10 non-hosts (all dual treatment, Fig. 1B). The third and fourth treatment were designed to investigate the interaction between accession and non-host herbivore presence. In the third treatment, all plants were infested with 10 hosts, while 10 P. xylostella caterpillars were added to the accession Christmas Drumhead (dual infested Christmas Drumhead, Fig. 1C). In the fourth treatment, all plants were infested with 10 hosts, while 10 P. xylostella caterpillars were added to the accession Badger Shipper (dual infested Badger Shipper, Fig. 1D). 


\section{A) Only hosts}

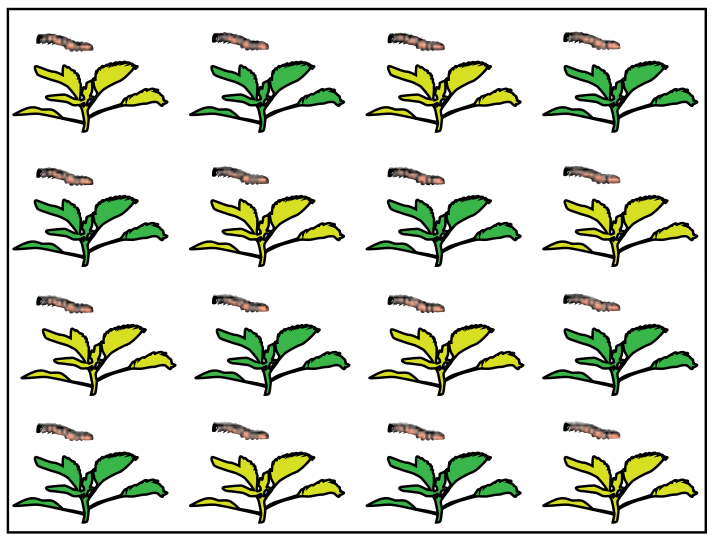

C) Christmas Drumhead dual
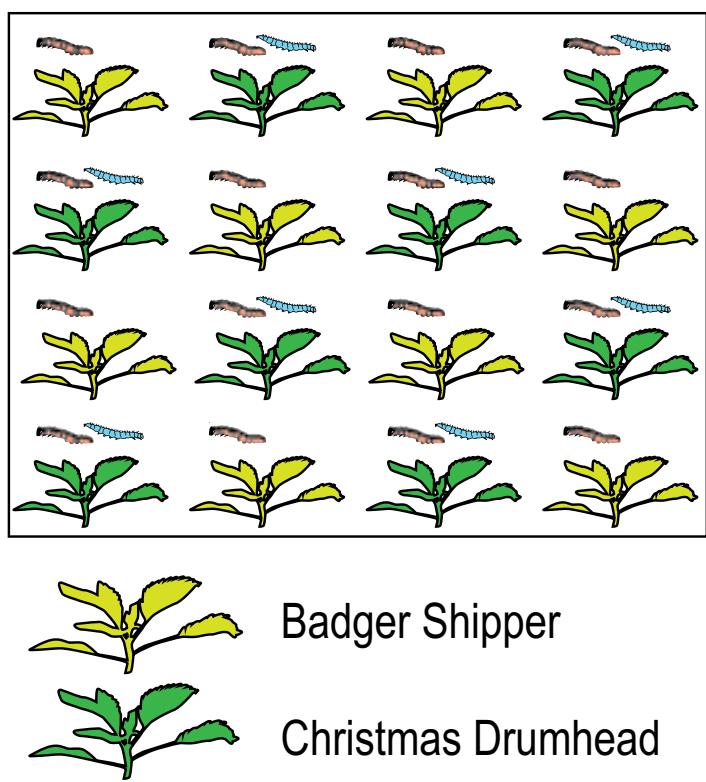

B) All dual

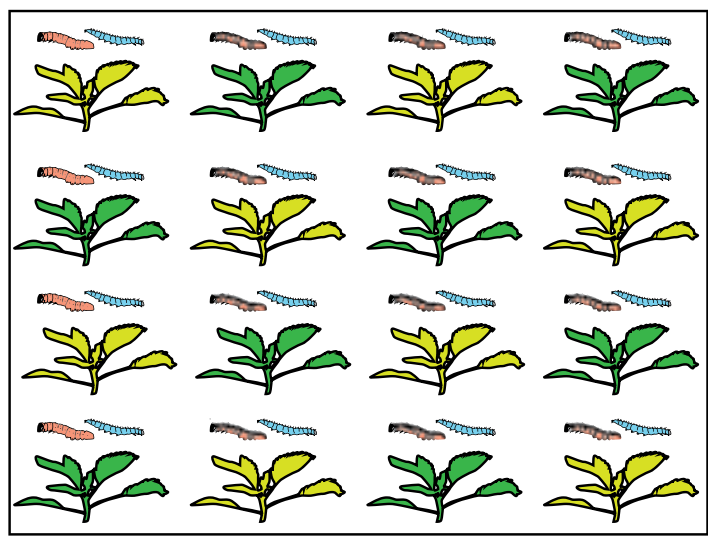

\section{D) Badger Shipper dual}

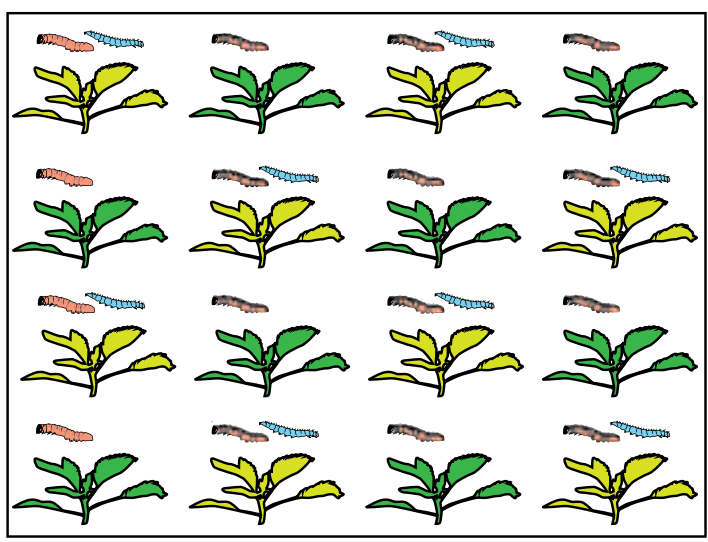

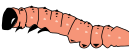

Pieris brassicae (host)

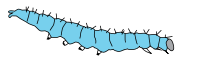

Plutella xylostella (non host)

Figure 1: Experimental set-up of the field-tent experiment. In mesh tents of $3 \times 4 \times 2 \mathrm{~m}(\mathrm{~L} \times \mathrm{W} \times \mathrm{H})$, eight plants of the accession Badger Shipper and eight plants of the accession Christmas Drumhead were planted. Each plant was infested with 10 L1 caterpillars of Pieris brassicae (host) while in dual infestation treatments, the plants were also infested with 10 L2 caterpillars of Plutella xylostella (non-host). Four comparisons were made: A) Only hosts on each plant; B) Hosts + non-hosts on each plant; C) Hosts on Badger Shipper versus hosts + non-hosts on Christmas Drumhead; D) Hosts + non hosts on Badger Shipper versus hosts on Christmas Drumhead. All four treatments were replicated five times during the summer of 2015. Each of the five times pertaining to one particular treatment was executed in three tents at the same time, resulting in a total of 15 replicate tents per treatment. 
After allowing the caterpillars to feed on the plants for $24 \mathrm{~h}$, three naïve female and three male C. glomerata were released in each tent. The males were added to make sure the female parasitoids were mated. A Petri dish with honey and water was placed in each tent providing the parasitoids with an ad libitum food source (Bukovinszky et al., 2012). One day after releasing the parasitoids, all plants were cut and placed individually in plastic bags, which were stored at $4{ }^{\circ} \mathrm{C}$. During the following days, the P. brassicae caterpillars were recovered from these plants to dissect them and check for parasitism by $C$. glomerata. All four treatments were replicated five times during the summer of 2015. Each of the five times pertaining to one particular treatment was executed in three tents at the same time, resulting in a total of 15 replicate tents per treatment.

\section{Wind tunnel assays}

To assess whether infestation with host, non-host or both herbivore species influences the preference of parasitoids for Badger Shipper or Christmas Drumhead in more detail, parasitoid choice behaviour was assessed in a wind tunnel (Geervliet et al., 1994). In nine dual-choice situations we assessed parasitoid preference for either a Badger Shipper or Christmas Drumhead plant with different herbivore infestation treatments. In three dual-choice situations, we assessed preference for one of the accessions when both plants had the same herbivore treatment of either 10 host caterpillars, 10 non-host caterpillars, or both (10 hosts and 10 non-hosts). In two situations, preference for host- or non-host infested plants was tested while switching the location of the host between the two accessions. Finally, we tested the preference for dual infestation vs host or non-host for either accession in another four dual-choice situations. Plants were infested with herbivores $24 \mathrm{~h}$ before the plants were used in the wind tunnel set-up. Caterpillars were initially placed on the same leaf, but could move freely on the plant.

Wind speed in the tunnel was set at $0.1 \mathrm{~m} \mathrm{~s}^{-1}$, under light conditions of 24 to $26 \mu \mathrm{mol} \mathrm{m} \mathrm{m}^{-2} \mathrm{~s}^{-1}$, temperature $21-24{ }^{\circ} \mathrm{C}$ and relative humidity above $50 \%$. A single parasitoid was transferred from the rearing cage to the wind tunnel in a glass tube. The tube was placed in a glass release cylinder at approximately 70 $\mathrm{cm}$ distance from the plants. From the moment the open tube containing the 
parasitoid was in the release cylinder, the parasitoids were given ten minutes to exit the tube, take off and fly to one of the plants. If a parasitoid did not make a choice within these ten minutes, it was removed and the trial was recorded as a "no response". If the parasitoid landed on one of the plants, the accession on which the parasitoid landed was recorded and the parasitoid was removed from the plant. We tested up to 10 naïve parasitoids per set of plants. After five consecutive tests, the position of the two plants was swapped. Four or five plant pairs were tested per treatment combination. After the assays, leaves of the two plants were scanned using Scion Image for Windows (Scion Corporation, Maryland, USA) to quantify the leaf damage by the caterpillars.

\section{Collection and analysis of VOCs}

To investigate whether parasitoid foraging decisions could be explained by differences in HIPV blends of the two accessions infested with different herbivores, plant volatiles were collected from plants of both accessions with either no herbivores (control), 10 L1 P. brassicae caterpillars (host), 10 L2 P. xylostella caterpillars (non-host) or both 10 L1 P. brassicae and 10 L2 P. xylostella (dual). For the collection of volatiles, plant individuals were different from those used in the wind tunnel experiment. Herbivores were placed on the plants $24 \mathrm{~h}$ before the experiment and were removed just before the collection of VOCs. Plants were given $50 \mathrm{~mL}$ of water before collection of VOCs.

Pot and soil were wrapped in aluminium foil, and subsequently, plant volatiles were collected in $28 \mathrm{~L}$ glass containers with rubber and PTFE closing elements. Plants were placed in the jars $\sim 40$ minutes before starting the collection to allow the plants to acclimatize. Volatiles were collected for 100 minutes with a flow of charcoal-filtered synthetic air (nitrogen $80 \%$, oxygen $20 \%$ ) pushed into the $28 \mathrm{~L}$ containers at a rate of $320-340 \mathrm{~mL} \mathrm{~min}{ }^{-1}$. Air was pulled from the containers at a rate of $302 \mathrm{~mL} \mathrm{~min}^{-1}$ using an air-sampling pump. Stainless steel tubes filled with $200 \mathrm{mg}$ Tenax TA (20/35 mesh size; Markes International, Llantrisant, $\mathrm{UK})$ were used to collect the dynamic headspace samples. Then, the tubes were dry-purged with helium for lowering their moisture content, and stored at room temperature until GC-MS analysis. After collecting the VOCs, the above-ground parts of the plants were cut and weighed. 
Eight plants per treatment were sampled. To minimize variation due to the circadian rhythm of the plants, samplings took place between 12 and $15 \mathrm{~h}$. One sampling event was conducted per day in a set-up that can accommodate simultaneous sampling from four plants in separate jars. Four or five collections were done weekly, and every treatment was sampled at least once every week.

The analysis of the VOCs was carried out by GC-MS in a thermal desorption (TD)-GC-MS instrument. The VOCs were desorbed from the sample TD tubes and re-adsorbed on an electrically-cooled sorbent trap. The compounds, desorbed from this trap, were transferred to the GC column. VOCs were ionized via electron impact, in a single-quadrupole mass spectrometer. Detailed information on the analysis of the VOCs-including GC column used and how compounds were putatively identified-is available in Supplementary Information 1.

\section{Data processing and statistical analyses}

We used generalized linear mixed models with binomial error distribution to analyse the data of the field tent experiment. We determined whether overall parasitism rates in the tents (not taking into account within-tent differences between accessions) differed among the four treatments. Data on Badger Shipper and Christmas Drumhead were pooled for each tent to get an overall parasitism rate. An observation level random effect was used to account for overdispersion. The response variable was either the number of parasitized caterpillars out of the total number of recollected caterpillars in a single tent, or the number of plants with at least one parasitized caterpillar out of the total number of plants from which caterpillars were recollected. The herbivore infestation treatment in the tent ("treatment") and the temporal replication of the experiment ("period") were fixed factors. The interaction between infestation treatment and time period was not significant and therefore dropped from the model.

Furthermore, we investigated within each treatment whether parasitism rates differed between the two accessions in four generalized linear mixed models with binomial error distribution. An observation level random effect was used to account for overdispersion in the data. The response variable was either 
the number of parasitized caterpillars out of the total number of recollected caterpillars on plants of each accession in a single tent, or the number of plants of each accession with at least one parasitized caterpillar out of the total number of plants from which caterpillars were recollected. Cabbage accession and time period of the experimental replicates were fixed factors. The interaction between accession and time period was not significant and subsequently dropped from the model.

A binomial test was used for each combination of plants and infestation type in the wind tunnel experiment to test the null hypothesis that the probability of choosing either plant in a pair was random $(\mathrm{P}=0.5)$. Additionally, Chi-Square tests of independence were used to compare distributions of preferences between Christmas Drumhead and Badger Shipper.

The chromatograms obtained from the GC-MS analyses were processed (peak picking, baseline correction and peak alignment) in untargeted manner using MetAlign software (Lommen, 2009). The raw processed file from MetAlign was further filtered by excluding mass features that were not detected in more than $50 \%$ of the replicates of at least one of the treatments. Using the filtered file, mass signals $(m / z$, mass-to-charge ratio) originating from the same metabolites were clustered based on retention window and their correlation across all measured samples using MSClust software (Tikunov et al., 2012). A total of 335 centrotypes (putative VOCs) were generated after the clustering of the mass signals. Three of the original 64 samples (eight replicates per control or treatment) were discarded due to infestation with thrips, resulting in a total of 61 samples. Eleven duplicate centrotypes and six centrotypes of centrotypefactors below 0.80 were deleted - the latter, aiming at decreasing the likelihood of having artefact-centrotypes-resulting in 318 centrotypes. Then, the mass signals of centrotypes displaying peak height values $>1.20 \times 10^{8}$ counts—even if in only one sample-were exchanged by mass signals of intensity $<1.20 \times$ $10^{8}$ counts across all samples, ensuring that that there was no saturation of the detector in all data used. 
Before performing statistical analysis on the VOCs data, peak height of every compound was divided by the biomass of the plant used in the experiment. The data were then log-transformed to correct for heteroscedasticity, and range-scaled to standardize the range of values of each compound (van den Berg, 2006). To allow log-transformation of data on compounds that were not detected in a sample, a value of 1 was added to all data points before transformation. Not all 318 centrotypes were actual VOCs. Out of those that were VOCs, many originate from background and analytical system. Compounds that were found to be significantly different in at least one of the treatment combinations by performing a Kruskal Wallis test (for non-normally distributed data) or ANOVA (for normally distributed data) $(\alpha=0.05)$ were subjected to post-hoc tests to determine which treatments differed. This was done via either Dunn's test of multiple comparisons (for non-normally distributed data) or Tukey's all-pair comparisons (for normally distributed data). The final list consists of 27 compounds. Nearly all of them were putatively identified, and are listed in Table 4 and Fig. 4. We used hierarchical cluster analysis to group the eight treatments according to composition of the blend of these 27 VOCs. For the cluster analysis, treatment averages of each compound were used to derive clustering according to the Ward method and using Euclidean distances in a dendrogram. Approximately unbiased (AU) probability values were calculated to assess uncertainty in the analysis (Suzuki \& Shimodaira, 2006).

All statistical analyses were performed in R using the packages lme4 for GLMM, pvclust for hierarchical clustering, FSA for Dunn's test, stargazer for table output and rcompanion (Bates et al., 2015; Suzuki \& Shimodaira, 2015; Dinno, 2017; Hlavac, 2018; Mangiafico, 2018; Ogle, 2018). 


\section{Results}

\section{Field tent experiment}

Across the five replicates in time, we recollected 6169 out of the $9600 \mathrm{P}$. brassicae caterpillars (64\%), of which 1940 were parasitized (31\%). Overall parasitism rates in the tents were not significantly different between most of the different infestation treatments, except for the tents where Badger Shipper was dual-infested and Christmas Drumhead was infested with hosts only, which had a lower parasitism rate (GLMM, Table 1, Fig. 2A). Parasitism rates were significantly influenced by the time point during the summer (GLMM, Table 1).

Within tents of the individual treatments, the percentage of parasitized caterpillars was significantly higher on accession Christmas Drumhead than on Badger Shipper in all treatments (GLMM, Table 2, Fig. 2A), except when Badger Shipper was dual-infested and Christmas Drumhead was host-infested (GLMM, Table 2, Fig. 2A). The proportion of plants with parasitized caterpillars showed similar trends as the percentage of caterpillars parasitized (GLMM, Table 3, Fig. 2B). However, there was no significant difference between the plant accessions in the percentage of plants with parasitized caterpillars when both were dualinfested whereas there was a significantly greater percentage of parasitized hosts on Christmas Drumhead than on Badger Shipper. 

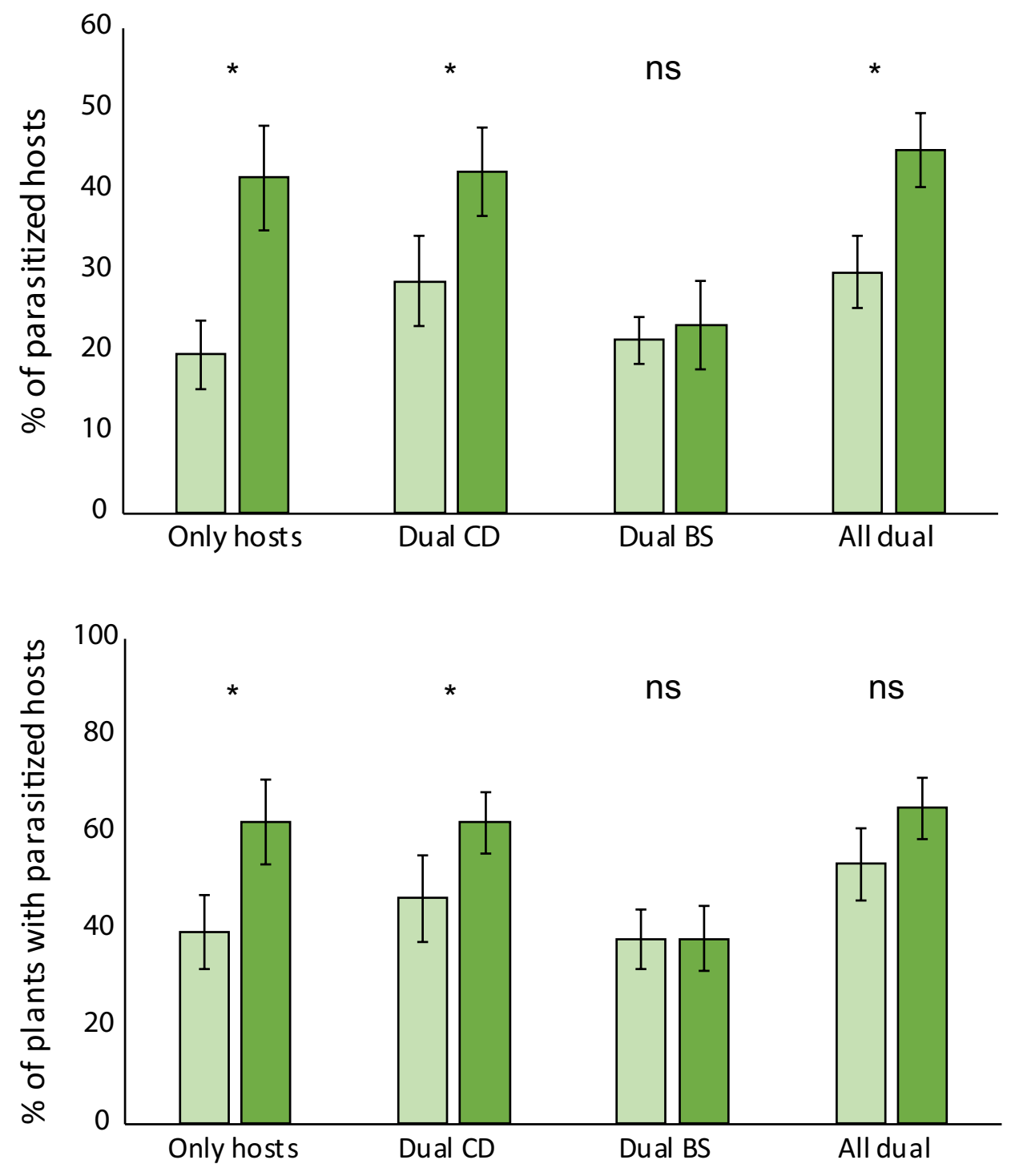

Figure 2: Parasitism rates (mean \pm SEM) of Pieris brassicae caterpillars by Cotesia glomerata parasitoids in a field-tent experiment with plants of the attractive accession Christmas Drumhead and plants of the less attractive accession Badger Shipper. Pairs of bars indicate results within a tent treatment and the colour represents plant accession. Plants were infested with either only P. brassicae caterpillars (hosts) or with both P. brassicae and the non-host Plutella xylostella (dual), displayed at the x-axis. ${ }^{*}$ indicates a significant difference between the varieties in a treatment (GLMM with binomial error distribution, $a=0.05$ ). ns = not significant. $\mathrm{N}=15$ per tent experiment. 
Table 1: Parameter estimates of generalized linear mixed models for overall parasitism levels (not taking into account within-tent differences between accessions). The dependent variable was either the number of parasitized caterpillars of the total number of $P$. brassicae caterpillars recovered from the tent, or the number of plants on which parasitized caterpillars were found out of the total number of plants in the tent (binomial error distribution, logit link). Fixed factors tested were treatment (four levels) and time point during the summer (five levels). There was no significant interaction between the two fixed factors and the interaction was therefore removed from the analysis. An observation level random effect was used to account for overdispersion. Estimates with standard error (between brackets) and statistical significance (bold and with asterisks) are reported $(a=0.05)$. Reference treatments are "Only hosts on both accessions" and "Trial 1".

Dependent variable:

Fixed factor $\downarrow \quad$ Level $\downarrow$

Constant

Treatment

Only hosts on both accessions

Hosts on Badger Shipper,

Dual on Christmas Drumhead

Dual on Badger Shipper,

Hosts on Christmas Drumhead

Dual on both accessions

Trial 1

Trial 2

Trial 3

Trial 4

Trial 5
$0.589(0.425)$

$0.542(0.496)$
$-0.021(0.369)$

Parasitized

caterpillars/total

recovered caterpillars in

tent

$-1.295^{* *}(0.383)$

$-0.820^{*}(0.374)$

$-0.438(0.372)$

$-0.432(0.437)$

tent

$-0.081(0.448)$

$-0.180(0.436)$

$-1.008^{*}(0.436)$
Plants with parasitized caterpillars/total number of plants in

$1.148^{* *}(0.424)$

$1.136^{*}(0.500)$

$0.388(0.424)$

$0.136(0.495)$

$0.963^{*}(0.427)$

$0.653(0.496)$ 
Table 2: Parameter estimates of generalized linear mixed models to assess differences between the two accessions within treatments in the number of parasitized Pieris brassicae caterpillars in the tent experiment. The dependent variable was the number of parasitized caterpillars out of the total number of $P$. brassicae caterpillars recovered from the tent (binomial error distribution, logit link). Fixed factors tested were accession ( 2 levels) and time point during the summer (5 levels). There was no significant interaction between the two fixed factors; therefore the interaction was removed from the analysis. An observation level random effect was used to correct for overdispersion. Estimates with standard error (between brackets) and statistical significance (bold and with asterisks) are reported $(\alpha=0.05)$. Reference treatments are "Badger Shipper" and "Trial 1".

Dependent variable:

Parasitized caterpillars/Total caterpillars recovered

CD dual, CD hosts,

Fixed factor $\downarrow \quad$ Level $\downarrow \quad$ Only hosts BShosts $\quad$ BS dual All dual

Constant

$-2.937^{* *}(0.725)-1.323^{* *}(0.426)-2.655^{* *}(0.602)-1.475^{* *}(0.469)$

Accession Badger Shipper

Christmas Drumhead $1.367^{*}(0.534) \quad 0.926^{* *}(0.343)-0.056(0.426) \quad 0.816^{*}(0.367)$

Time $\quad$ Trial 1

Trial $2 \quad-0.126(0.888) \quad 0.227(0.535) \quad 1.215(0.721) \quad 0.833(0.588)$

Trial $3 \quad 1.869^{*}(0.869) \quad 1.031(0.533) \quad 1.564^{*}(0.724) \quad 0.444(0.590)$

Trial $4 \quad 1.263(0.870) \quad-1.324^{*}(0.550) \quad 1.681^{*}(0.718)-0.137(0.590)$

$\begin{array}{lllll}\text { Trial } 5 & 1.637(0.873) & 0.360(0.548) & 1.086(0.738) & 0.747(0.591)\end{array}$

${ }^{*} \mathrm{p}<0.05 ; \stackrel{* *}{\mathrm{p}}<0.01$ 


\section{Wind tunnel experiment}

Of the 424 parasitoids released in the wind tunnel experiments with the different plant pairs, $250(59 \%)$ made a choice and landed on one of the plants within ten minutes. Over all plant combinations, Christmas Drumhead was the preferred accession with $66 \%$ of first landings (binomial test, $P<0.001$, Fig. 3 ). There was no preference for either accession when plants were infested with only hosts (binomial test, $P=1$, Fig. 3A). When both plants were infested with non-host caterpillars, more parasitoids landed on the accession Christmas Drumhead first (binomial test, $P<0.001$, Fig. 3A). When both plants where dually infested, there was a tendency towards more landings on the accession Christmas Drumhead (binomial test, $P=0.10$, Fig. 3A). In the plant-pair combinations where one plant was infested with hosts and the other with non-hosts, parasitoids landed more often on the host-infested plant than on the non-host-infested plant, irrespective of the plant accession (binomial test, $p<0.001$ and $p<0.041$, Fig. 3B). However, when the hosts were on Badger Shipper and non-hosts on Christmas Drumhead, more parasitoids chose the non-host-infested plant compared to the reverse situation (Chi-square test, $\chi^{2}=4.12, d f=1, p=0.042$, Fig. 3B).

In plant pairs with one dual-infested plant versus one host-only-infested plant, parasitoids landed more often on the dual-infested Christmas Drumhead compared to the host-only-infested Badger Shipper (binomial test, $p=0.011$, Fig. 3C), but did not land more often on dual-infested Badger Shipper as compared to host-onlyinfested Christmas Drumhead (binomial test, $p=0.26$, Fig. 3C). The proportion of parasitoids choosing the Christmas Drumhead plant did not differ between the two combinations (Chi-square test, $\chi^{2}=0.935, d f=1, p=0.333$, Fig. 3C). When the accession Christmas Drumhead was dually infested and the accession Badger Shipper was infested with only non-hosts, parasitoids preferred to land on the dually infested plant (binomial test, $p<0.001$, Fig. 3C). However, when the accession Badger Shipper was dually infested and Christmas Drumhead only infested with non-hosts, parasitoids had no preference for either plant (binomial test, $p=0.15$, Fig. 3C). Comparing the two non-host vs dual combinations, choice for either dual or non-host infested plants was influenced by the accession on which the herbivores were present, with more parasitoids choosing the non-host-infested plant if it was Christmas Drumhead (Chi-square test, $\chi^{2}=8.62, d f=1, p=0.0033$ ). 
Table 3: Parameter estimates of a generalized linear mixed models for comparing within treatments the number of plants with parasitized Pieris brassicae caterpillars in the tent experiment. The dependent variable was the number of plants on which parasitized caterpillars were found out of the total number of plants in the tent (binomial error distribution, logit link). Fixed factors tested were accession ( 2 levels) and time point during the summer ( 5 levels). There was no significant interaction between the two fixed factors; therefore it was removed from the analysis. An observation level random effect was used to limit overdispersion. Estimates with standard error (between brackets) and statistical significance (bold and with asterisks) are reported ( $\alpha=$ 0.05). Reference treatments are "Badger Shipper" and "Trial 1".

\section{Dependent variable:}

Plants with parasitized hosts/Total number of plants

Fixed factor $\downarrow$ Level $\downarrow$

Constant

Accession Badger Shipper

Christmas Drumhead

$1.365^{*}(0.646)$
Only hosts

$C D$ dual, $B S$ hosts

$-1.642(0.840)$

$-0.387(0.514)$
$C D$ hosts, $B S$

dual
All dual

$-0.01(0.579)$

$0.840^{*}(0.424)-0.018(0.409)$

$0.564(0.462$

Time

Trial 1

Trial 2

$-0.122(1.029)$

$0.456(0.642)$

$0.957(0.677)$

$0.817(0.744$

Trial 3

$1.906(1.037)$

$1.906^{* *}(0.709)$

$0.934(0.676)$

$0.018(0.731$

Trial 4

$1.318(1.026)$

$-1.302(0.673)$

$0.802(0.671)$

$0.281(0.737$

Trial 5

$1.768(1.047) \quad-0.073(0.652)$

$0.811(0.679)$

$0.281(0.737$ 


\section{Badger Shipper $\quad \square$ Christmas Drumhead}

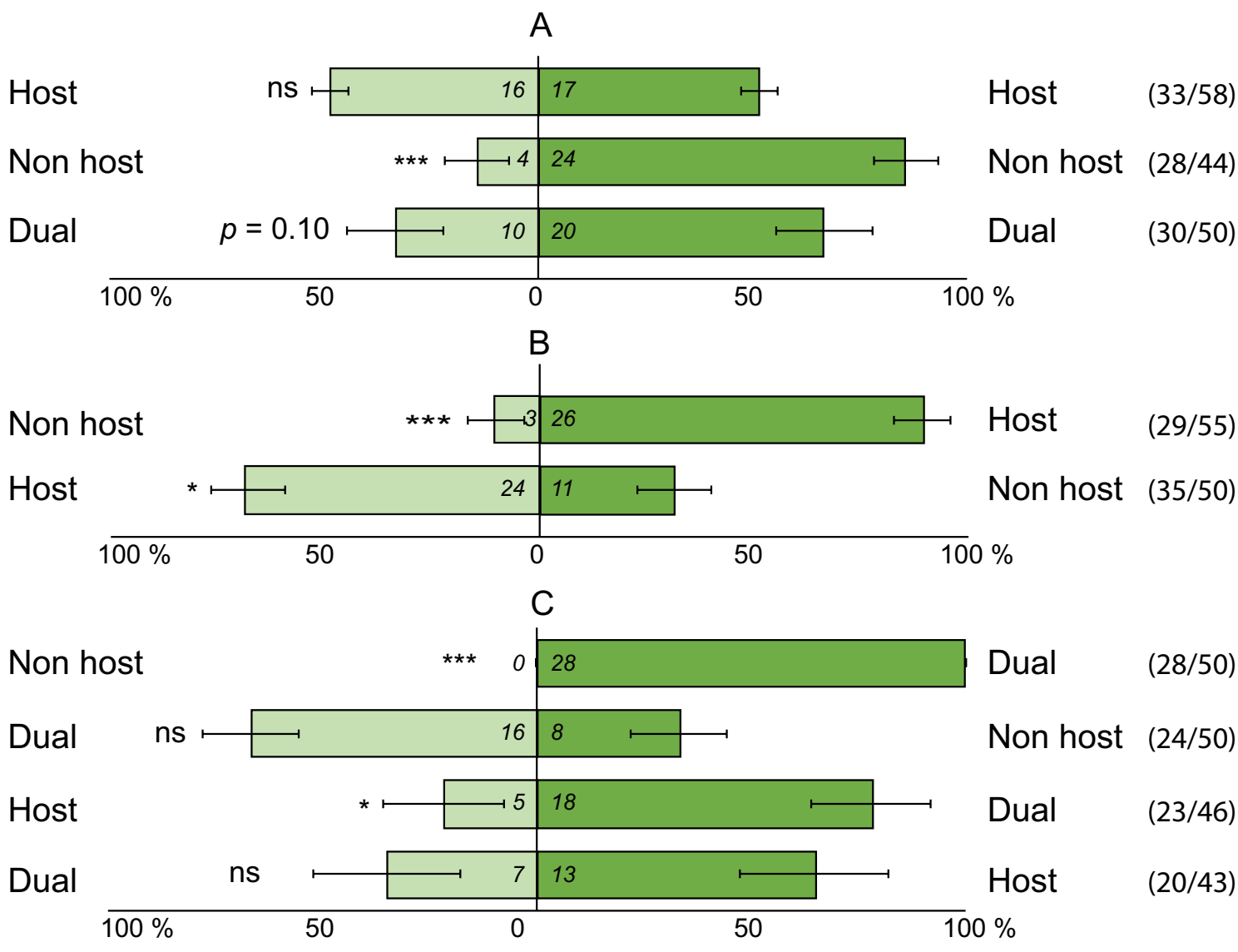

Figure 3: Results of a wind tunnel experiment in which Cotesia glomerata females were offered a plant pair consisting of one Badger Shipper and one Christmas Drumhead plant, with different caterpillar infestation treatments. Plants were infested with ten caterpillars of the host Pieris brassicae, ten caterpillars of the non-host Plutella xylostella, or a combination of 10 individuals of both herbivore species. A) Equal herbivore infestation on the two accessions. B) Non host vs. host on either accession. C) Dual vs. host or non-host for either accession. Five plant pairs were tested for each combination. Each bar represents the proportion of parasitoids choosing the respective plant, and the error bars represent the variation (SEM) between plant pairs. Numbers within the bars represent the number of parasitoids choosing that accession, while the numbers in brackets on the right represent the number of parasitoids making a choice within ten minutes out of the total number of parasitoids tested. Preference for a plant within a combination was tested using a binomial test, significant preferences are indicated with asterisks $\left({ }^{*} P<0.05,{ }^{* *} P<0.01,{ }^{* * *} P<0.001, n s=\right.$ not significant). 
Leaf damage after $24 \mathrm{~h}$ of feeding was influenced by herbivore species, but not by cabbage accession (Kruskal-Wallis chi-squared $=79.506, d f=2, p<0.001$ and Kruskal-Wallis multiple comparisons, Supplemental Information 2 Fig. 1). Leaf damage by ten $P$. xylostella caterpillars was less than that by ten $P$. brassicae caterpillars or a combination of ten P. xylostella and ten P. brassicae (Supplemental Information 2; Fig. 1). There was no difference in leaf damage between plants infested with ten P. brassicae and dual-infested plants (Supplemental Information 2 Fig. 1).

\section{Collection and analysis of VOCs}

There were 27 compounds that differed significantly in peak heights $\mathrm{g}^{-1}$ biomass between at least two treatments in pair-wise comparisons (Kruskal Wallis or ANOVA, Table 4). Cluster analysis revealed that both cultivars produced different blends of these compounds and that for Badger Shipper two clusters can be discerned, one for clean plants and host-infested plants, and another one for non-host and dual-infested plants (Fig. 4). For Christmas Drumhead, the blend of the 27 VOCs of clean plants was different from that of all three types of herbivore-induced plants, but within herbivore treatments no significant clustering was apparent (Fig. 4). Pairwise comparisons between treatments for single compounds show that the largest differences occur between cultivars, and between control and dual infested plants (Fig. 4, Table 4). 


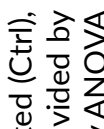

嵌

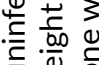

这

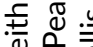

宁荷

U人

$\bar{\sigma}_{0}$

要

है을

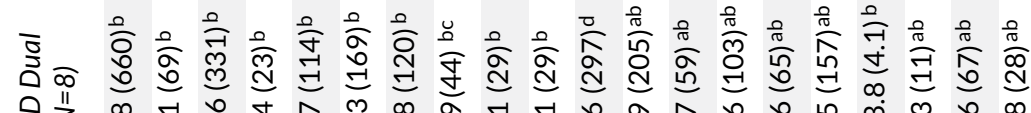

४ 3 少

in

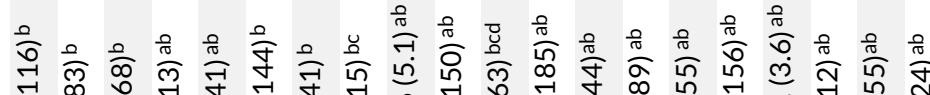

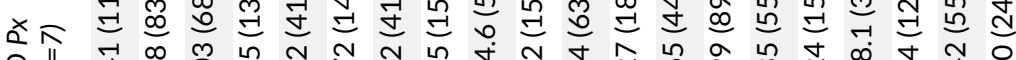

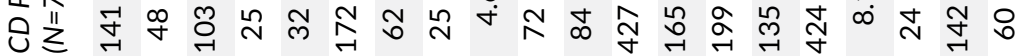

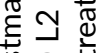

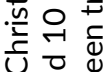

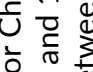

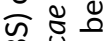

乐

बे

을

ज牙

هํ.

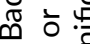

$\tilde{c} \cdot \bar{x} \cdot \frac{000}{n}$

은

凹్

$\breve{\sigma}$

o

艺 产

논

중 공

공을

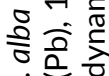

宊 8.0

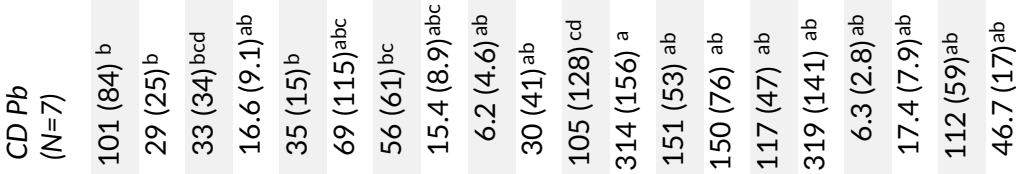

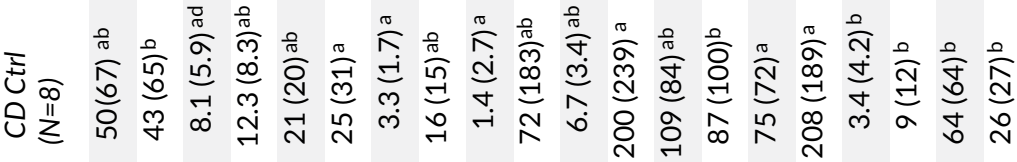

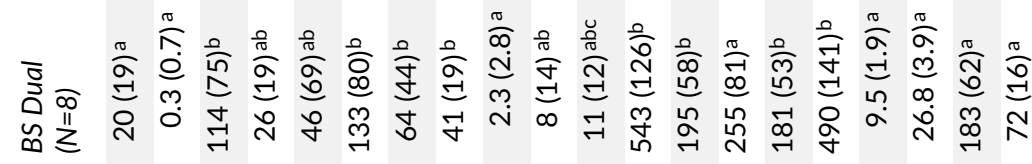

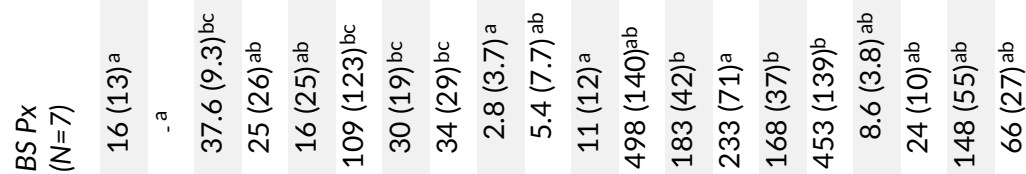

赵

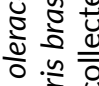

氜.

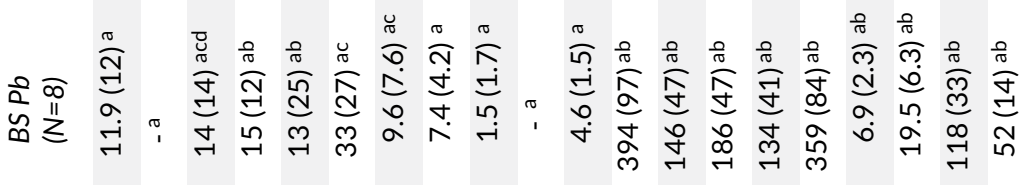

ชัง

응

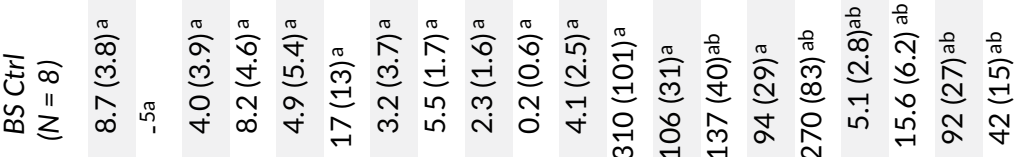

总㐫苛

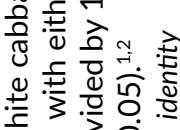

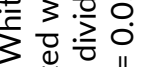

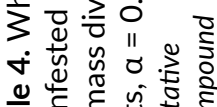

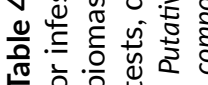

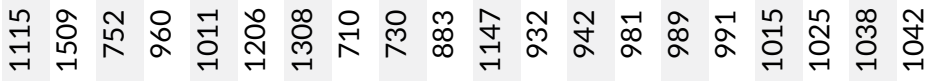

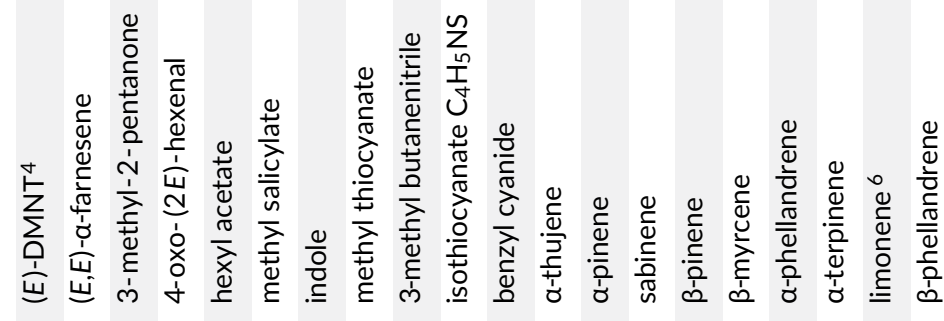




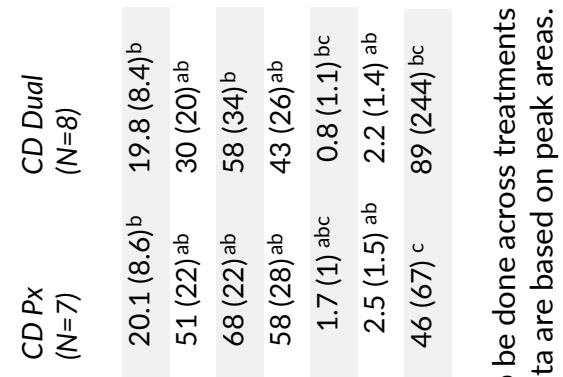

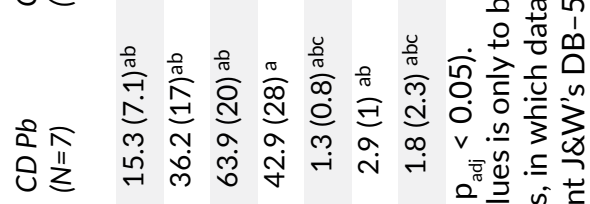

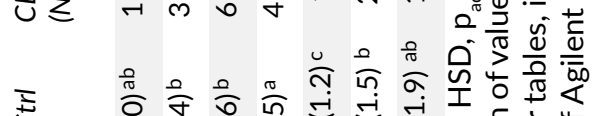

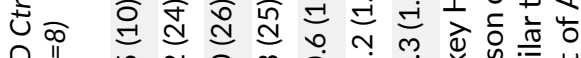

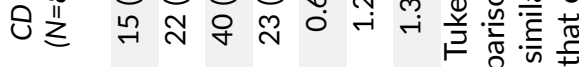

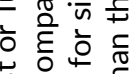

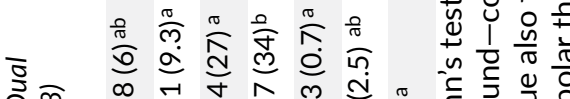

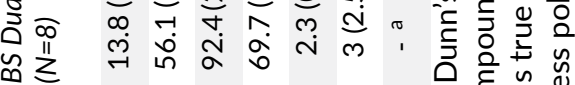

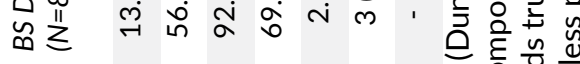

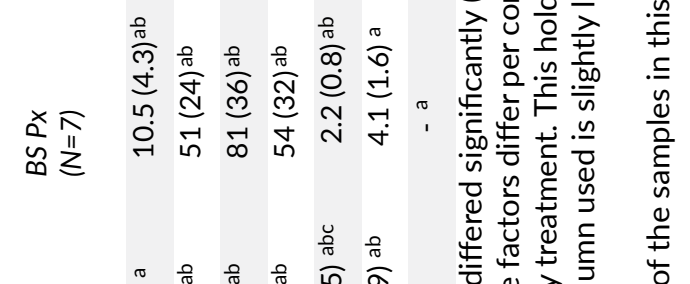

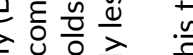

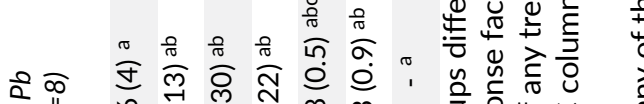

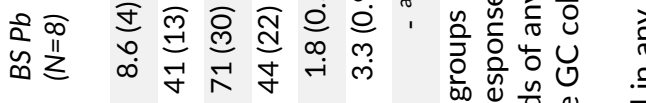

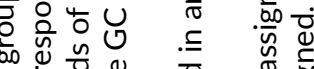

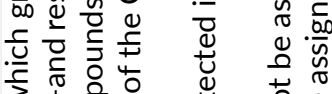

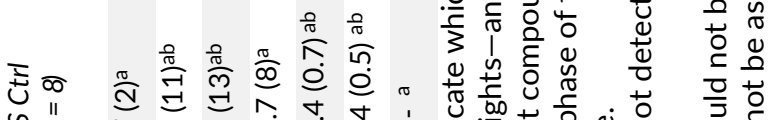
品之

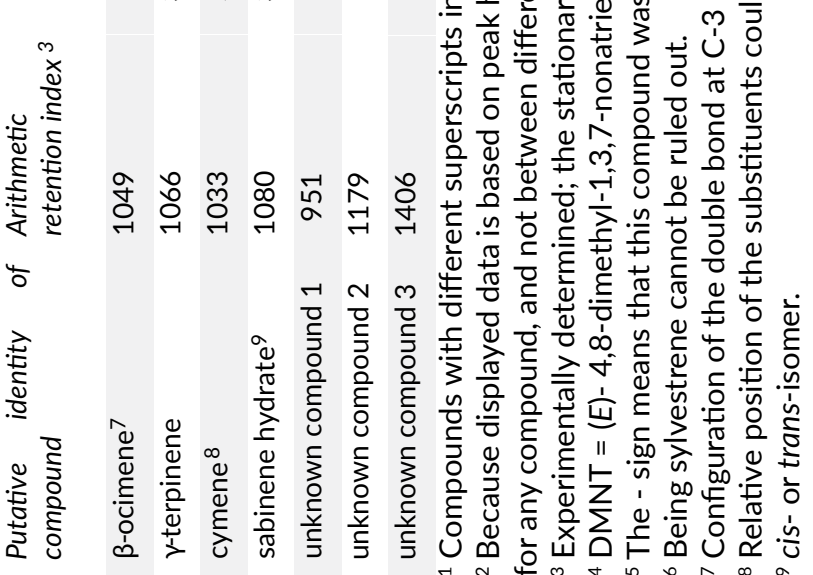



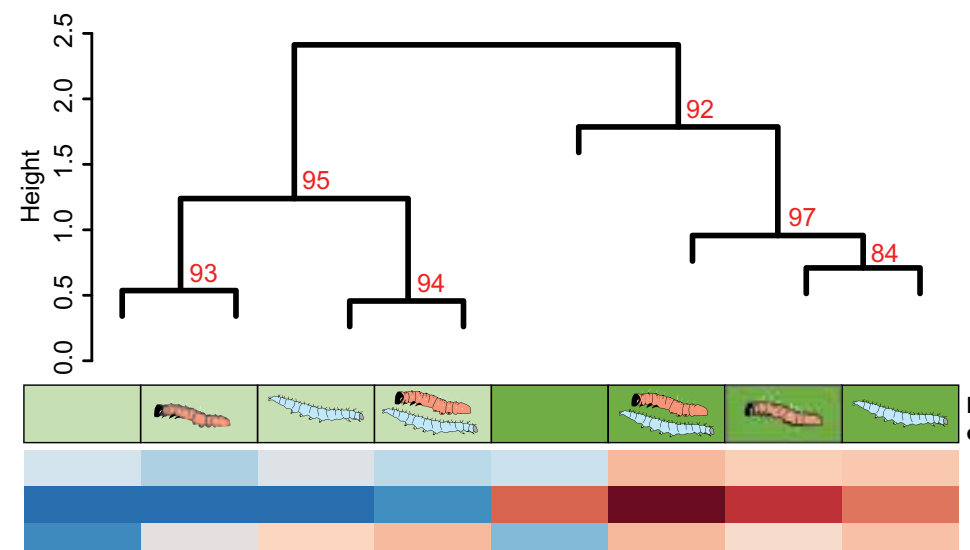

Putative identity of compound:

(E)-DMNT

$(E, E)$ - $\alpha$-farnesene

3-methyl-2-pentanone 4-oxo-(2E)-hexenal hexyl acetate methyl salicylate indole methyl thiocyanate 3-methyl butanenitrile isothiocyanate $\mathrm{C}_{4} \mathrm{H}_{5} \mathrm{NS}$ benzyl cyanide a-thujene a-pinene sabinene $\beta$-pinene $\beta$-myrcene $\alpha$-phellandrene a-terpinene limonene $\beta$-phellandrene $\beta$-ocimene $\gamma$-terpinene cymene sabinene hydrate unknown compound 1 unknown compound 2 unknown compound 3

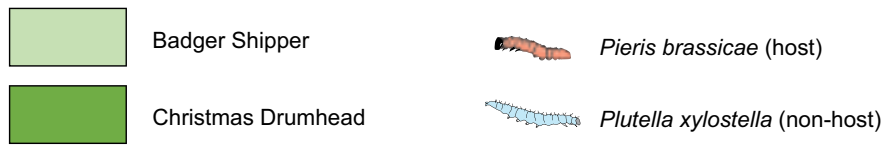

Figure 4: Cluster analysis and heat map of 27 volatile organic compounds emitted by white cabbage plants that significantly differed in at least one of the treatment groups. Values of peak height divided by biomass were log-transformed and range-scaled across all treatments before analysis. Putative identity of the compounds is shown on the right. Colour intensity is based on scaled data, meaning that their interpretation is relative to the range of values of each compound across treatments and not directly relating to actual amounts of any particular compound. Numbers displayed in red in the dendrogram represent approximately unbiased probability values for the clustering. 


\section{Discussion}

Our data show that plant-trait variation in terms of attractiveness influences parasitoid preference and host-finding success, confirming the earlier findings that Christmas Drumhead is a more attractive accession than Badger Shipper (Poelman et al., 2009b). However, plant-trait variation interacts with the presence of a non-host caterpillar in determining parasitoid host-finding success. When a less attractive accession was dually infested (with hosts and non-hosts for the parasitoid) and the more attractive accession was host-infested, overall hostfinding success was reduced compared to the reverse situation and situation with only hosts, and parasitism rates became equal on each accession. However, when both accessions were dually infested, host-finding was not reduced compared to the other situations and the more attractive accession had higher parasitism rates than the less attractive accession. This indicates that dual herbivory on one plant can affect parasitism rates on neighbouring plants which are not dually infested. Here we discuss two possible mechanisms for this finding: (i) alteration of HIPV cues by non-host herbivores specific for plant genotypes, and (ii) onpatch processes when parasitoids search for hosts on dual herbivore infested plants.

In other studies, parasitoids often did not discriminate between plants infested with host and non-host caterpillars (Shiojiri et al., 2000; Bukovinszky et al., 2012; Peñaflor et al., 2017). In case of sucking herbivores, such as aphids as non-hosts, parasitoids are more able to discriminate, suggesting that the different feeding modes lead to different cues from the plant (Powell et al., 1998; de Rijk et al., 2016b). However, the species identity of the chewing herbivore also matters for a parasitoid's ability to distinguish host from non-host infested plants (Geervliet et al., 1998; de Rijk et al., 2016a). As we did not test preference for host or non-host infested plants within the same accession, the question whether parasitoids can discriminate between volatiles of plants of these two accessions with different herbivores remains. However, we show that the outcome of non-host herbivory for parasitoid foraging efficiency can also be affected by traits of the plants on which these herbivores are feeding. 
Plants can respond to herbivores in a species-specific manner (Clavijo McCormick et al., 2012), resulting in the emission of different odour blends after herbivory by different herbivore species. There can be inter- and intraspecific variation in plant responses to specific combinations of herbivores (Turlings et al., 1998; Gols et al., 2011). Therefore, some plant varieties may produce more distinctly different odour blends after feeding damage by different herbivores than others. The two accessions used in this study produced clearly distinct volatile blends, and also within accessions induction by different herbivores resulted in specific changes in volatile blends. For the less attractive accession Badger Shipper, the volatile blend of control plants and plants infested with the host $P$. brassicae does not change much. The herbivore $P$. xylostella has a slightly larger effect on the volatile blend when either present alone or in combination with P. brassicae than P. brassicae alone. For the more attractive accession Christmas Drumhead, volatile blends of uninfested plants were clearly different from herbivore-induced plants, but blends of herbivore-induced plants were similar, irrespective of the identity of the herbivore. This might explain why non-host infested Christmas Drumhead plants in the wind tunnel were distracting parasitoids from landing on host-infested Badger Shipper plants.

While many generalist predators use generic HIPVs, such as green leaf volatiles or terpenoids, specialist parasitoid species may use HIPVs which are less common in a blend, but may be more indicative of the host plant or herbivore species (Turlings \& Erb, 2018). For example, parasitoids which are searching for herbivores on Brassica plants may respond to glucosinolate (GLS) breakdown products, which are specific for that plant genus (Mumm \& Dicke, 2010; Turlings \& Erb, 2018). The accession Christmas Drumhead had higher emissions of the GLS-breakdown product isothiocyanate in both control and herbivore-infested plants, and the GLS-breakdown product benzyl cyanide was induced in herbivore-infested Christmas Drumhead. The latter has been shown to be present in volatiles from infested B. oleracea var gemmifera plants and might play a role in the attraction of C. glomerata (Scascighini et al., 2005).

Methyl salicylate was emitted more in plants induced with P. xylostella and dual infested plants of both varieties than in control plants and plants induced with $P$. 
brassicae. Previous work using these accessions and the herbivore P. rapae indicated that emission of methyl salicylate was increased in Christmas Drumhead after herbivory, but not in Badger Shipper (Poelman et al., 2009b). This indicates that both herbivore identity and plant accession influences HIPV blends, because one herbivore may induce different changes in different accessions, and different herbivores may induce different changes in the same accession. It is possible that with regard to this compound, dual-infested Badger Shipper plants became more similar to host-infested Christmas Drumhead.

Although differences in volatile blends as a result of herbivory are common, it usually remains unclear which compounds are used by parasitoids and influence parasitoid foraging behaviour (Gols et al., 2011; Gols et al., 2012). Furthermore, parasitoids may respond to mixes of compounds, rather than isolated compounds, and background odours may also play a role (Schröder \& Hilker, 2008; van Wijk et al., 2011). The differences in volatile profiles of the two accessions as a result of infestation with different herbivores do not give a conclusive explanation for the findings in the behavioural assays of this study. Additionally, while the cluster analysis created groups of treatments with regard to the volatile blend, individual compounds often did not differ between herbivore treatments due to the large variation between samples of the same treatment. Cluster analysis used the compounds which differed between the plants and not only compounds which are actually used (separate or in a blend) by C. glomerata to find hostinfested plants. If all these compounds were known, cluster analysis on a subset of these compounds might result in different clustering which may explain more of the preference hierarchy of C. glomerata for host and non-host infested accessions found in the wind tunnel tests.

Besides alteration of HIPV blends by non-host herbivores and their effect on parasitoid choice behaviour, on-patch foraging behaviour might also explain why host-finding efficiency is different under dual infestation. Several stages in parasitoid foraging behaviour can be distinguished: patch searching, hostsearching within patch and host selection (van Alphen et al., 2003). Parasitoids can use different cues during different stages of foraging, which includes cues from the host plant and cues from the herbivore itself. In the tent 
experiment, besides choosing which plant to land on, on-patch decisions can affect host-location success (van Alphen et al., 2003; de Rijk et al., 2013). Nonhost herbivores may affect on-patch foraging behaviour in several ways. For example, non-host presence might result in short giving-up time upon landing in a patch (Bukovinszky et al., 2012). However, when both hosts and non-hosts are present on a plant, giving-up time is not shorter than in host-only situations (Bukovinszky et al., 2012). Parasitoids can waste time on following direct cues from non-hosts such as feeding sites or frass (Takabayashi \& Takahashi, 1990), and even start ovipositing in non-hosts (Bukovinszky et al., 2012; Chabaane et al., 2015). It is possible that more landings on dual-infested Badger Shipper caused the parasitoid to waste time on non-host encounters and that they therefore visited fewer plants in the tents. However, this does not explain why the reverse situation with dual-infested Christmas Drumhead and the situation in which all plants were dual-infested did not have this reduction in parasitism rates. Possibly, on-plant foraging behaviour and on-plant cues are different for dual-infested Badger Shipper and Christmas Drumhead plants and this may explain better what happens during host-searching on the plant.

Parasitoids are able to adapt foraging behaviour after oviposition experience by learning to associate cues with presence of their hosts (Perfecto \& Vet, 2003; Smid et al., 2007; Hoedjes et al., 2011). Ability to associate cues with oviposition may be more difficult depending on context. In the tent-experiment, all plants were infested with hosts. Therefore, it is unlikely for a parasitoid to have a negative learning experience by landing on a plant which contains non-hosts. Such incomplete information may have prevented parasitoids to optimize their foraging strategy (Vet et al., 1998; Erb et al., 2010; de Rijk et al., 2018). Therefore, parasitoids may have kept returning to the dual-infested Badger Shipper where they may waste time on non-hosts. When all plants contain non-hosts, they may do the same on the more attractive Christmas Drumhead. Experience with hosts can also lead to an increase in tendency to search for more hosts, especially for parasitoids foraging for gregarious hosts such as P. brassicae (Vos et al., 1998; Smid \& Vet, 2016). Encounters with a patchily distributed host may lead to reduced leaving tendency and therefore longer patch exploitation (Vos et al., 1998). Exposure to non-hosts might interfere with this process, but to what 
extent this is the case might depend on the cues presented with the non-hosts, such as different volatiles from plants or on-plant cues.

To date, no studies have examined the combination of plant trait variation and plant induction by hosts and non-hosts and their interactive effect on parasitoid behaviour in environments with multiple plant accessions and herbivore species. In field situations, it is likely that hosts and non-hosts occur across the landscape on different plant species and varieties within species. We have shown that both aspects influence parasitoid foraging behaviour, and that the outcome is not easy to predict based on previous work on either plant trait variation or non-host presence. In field situations, the environment in which a parasitoid is searching for hosts is heterogeneous and consists of many plants emitting different volatiles, resulting in a complex volatile mosaic (Aartsma, et al. 2017 and references therein). Besides structural variation from vegetation composition, patch size or landscape structure, the presence of non-host herbivores can impair hostlocation efficiency.

Changes in foraging efficiency through the presence of non-hosts or non-prey can result in more stable coexistence of species and prevent overexploitation by parasitoids and predators (Fan et al., 2010; Hammill et al., 2015). In other cases, non-hosts can increase attraction of parasitoids, for example in the case of parasitoid attraction to dual herbivore infested plants, which may result in stronger parasitism pressure on the host. These changes may be species-specific and can be mediated by other trophic levels as well, such as the plant. Our research shows that effects of plant-trait variation and non-host herbivores are not only resulting from HIPV-mediated foraging behaviour, nor only from on-patch decisions. Future research should include both variation in non-host herbivores and plant trait variation to further investigate the mechanisms of parasitoid foraging behaviour in complex situations and to find generalities in how these aspects interact. Especially focussing on the role of on-patch foraging and time spent on plants and handling non-hosts and hosts may shed light on how plant trait variation and variation in non-host herbivores interact. 


\section{Supplemental Information 1}

Analysis of VOCs by GC-MS

Analysis of VOCs was carried out in a thermal desorption (TD)-GC-MS instrument, consisting of a TD system with autosampler (Ultra TD and Unity modules; Markes International), a gas chromatograph (Trace GC Ultra; Thermo Electron), and a mass spectrometer (EI-single quadrupole Trace DSQ; Thermo Electron). The instrument is managed through Thermal Desorption System Control Program (Markes International) and Thermo Xcalibur (Thermo Fisher Scientific) software. In the TD system, the sample TD tubes were first purged at ambient temperature at a flow rate of $20.0 \mathrm{~mL} \mathrm{~min}^{-1}$ (3.0 min dry-purge and 1.0 min pre-purge; trap off line). During the primary desorption, VOCs were desorbed from the tubes for $10 \mathrm{~min}\left(20.0 \mathrm{~mL} \mathrm{~min}^{-1} ; 250^{\circ} \mathrm{C}\right)$. They were then re-adsorbed on an electrically-cooled sorbent trap $\left(20.0 \mathrm{~mL} \mathrm{~min}^{-1} ; 0{ }^{\circ} \mathrm{C}\right.$; pre-trap fire purge of $1.0 \mathrm{~min})$. The compounds were desorbed from this trap during the secondary desorption $\left(20.0 \mathrm{~mL} \mathrm{~min}^{-1}\right.$; heating rate of $40^{\circ} \mathrm{C} \mathrm{s}^{-1} ; 280$ ${ }^{\circ} \mathrm{C}$ for $10 \mathrm{~min}$ ), and were transferred to the $\mathrm{GC}$ column in splitless mode. The temperature of the sample flow path was set to $185^{\circ} \mathrm{C}$. The chromatographic process was carried out at a flow rate of the carrier gas of $1.0 \mathrm{~mL} \mathrm{~min}{ }^{-1}$. The oven temperature was programmed to go from $40{ }^{\circ} \mathrm{C}$ ( 2 min hold time) to $280{ }^{\circ} \mathrm{C}$ (4 min hold time) at $6{ }^{\circ} \mathrm{C} \mathrm{min}^{-1}$. This resulted in a $46 \mathrm{~min}$ temperature programme. The MS transfer line was set to $275^{\circ} \mathrm{C}$. [Note For the analysis of the mixture of alkanes: Oven programme was the same until 46 min; then, the temperature was raised to $300{ }^{\circ} \mathrm{C}$ over $1 \mathrm{~min}$ and kept at this temperature for 20 min.] The energy of the electron beam was set to $70 \mathrm{eV}$, and the temperature of the ion source was set to $250{ }^{\circ} \mathrm{C}$. The mass spectrometer scanned $m / z 35-400$ at a rate of 4.7 scans s$^{-1}$. [Note For the analysis of the mixture of alkanes: Settings were the same, except for a 6 min-delay before the beginning of scanning (due to solvent).] The GC column used was a ZB-5MS Zebron (Phenomenex); 30 $\mathrm{m} \times 0.25 \mathrm{~mm} \times 1.00 \mu \mathrm{m}$, with $10 \mathrm{~m}$ Guardian End. The stationary phase of this column consists of $5 \%$ polysilarylene- $95 \%$ dimethylpolysiloxane, making it slightly less polar than Agilent J\&W's DB-5. Helium was the gas used for desorption and chromatographic processes. 
A standard mixture of linear alkanes was analysed for the determination of the retention index (arithmetic index) values of the VOCs. A working solution of linear alkanes was prepared by diluting a commercial mixture of the compounds (C7-C30; Supelco) with petroleum ether 40-60 (puriss. p.a.; Sigma-Aldrich) $\sim 100 \times$. The concentration of each alkane in the working solution was $\sim 10 \mu \mathrm{g}$ $\mathrm{mL}^{-1}$. Approximately $0.5 \mu \mathrm{L}$ of this working solution was added to a TD tube using a $10 \mu \mathrm{L}$-glass syringe. This was followed by dry-purging of the tube $(\sim 25$ psi of helium, for $60 \mathrm{~min}$ at room temperature). Such a tube was analysed with the sample TD tubes.

All TD tubes were analysed within four days. The performance of the mass spectrometer was checked (maintenance tune and leak check) before the analytical sequence started, as well as after it ended.

Putative identification of the VOCs that were significantly different across controls/treatments Arithmetic retention index (AI) values of the VOCs were determined from their retention times and those of the linear alkanes, through the following formula (Adams, 2001):

$\mathrm{AI}=\left(100 * \mathrm{C}_{\mathrm{z}}\right)+\left\{100 *\left[\left(\mathrm{RT}_{\mathrm{x}}-\mathrm{RT}_{\mathrm{z}}\right) /\left(\mathrm{RT}_{\mathrm{z}+1}-\mathrm{RT}_{\mathrm{z}}\right)\right]\right\}$

in which $\mathrm{C}_{\mathrm{z}}$ is the number of $\mathrm{C}$-atoms of the alkane eluting just before the VOCs of interest (z), RT $T_{x}$ is the retention time of the VOC of interest (x), RT is the retention time of $z$, and $\mathrm{RT}_{z+1}$ is the retention time of the alkane eluting just after $\mathrm{x}$.

Putative identification of VOCs, generally: Experimentally acquired/MSClustreconstructed mass spectra were compared with those of libraries, taking retention index values into account; in-house knowledge on VOCs from plants was used too. Wageningen Mass Spectral Database of Natural Products and the MS library published by Adams (2001) were chiefly used. NIST Mass Spectral Search Program (U.S. Secretary of Commerce) was used. 


\section{Supplementary Information 2}

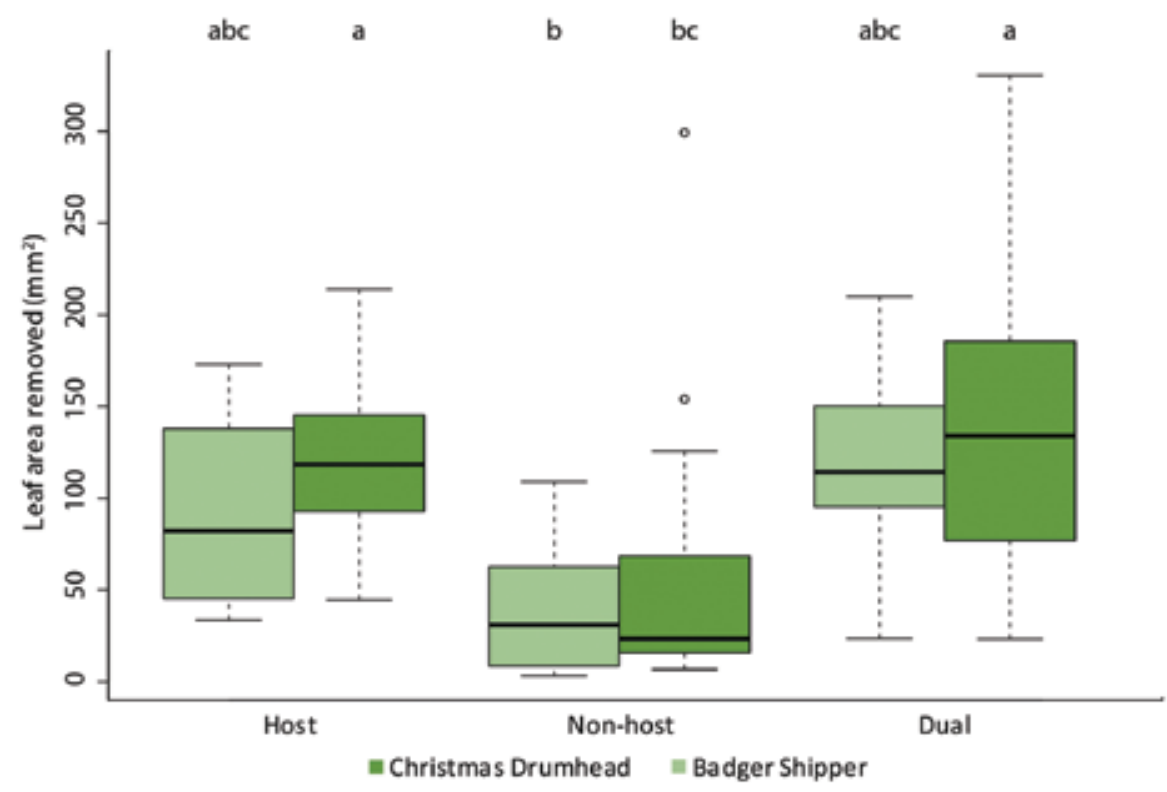

Figure 1: Leaf damage $\left(\mathrm{mm}^{2}\right)$ on leaves of white cabbage of the accessions Christmas Drumhead and Badger Shipper, damaged by either ten Pieris brassicae (host of Cotesia glomerata), ten Plutella xylostella (non-host) or by both ten P. brassicae and ten P. xylostella (dual). Box plot represents the median of leaf area removed. Different letters above the graph represent significant differences between the treatments (Kruskal-Wallis multiple comparisons, $a=0.05)$. 


\section{Chapter 6}
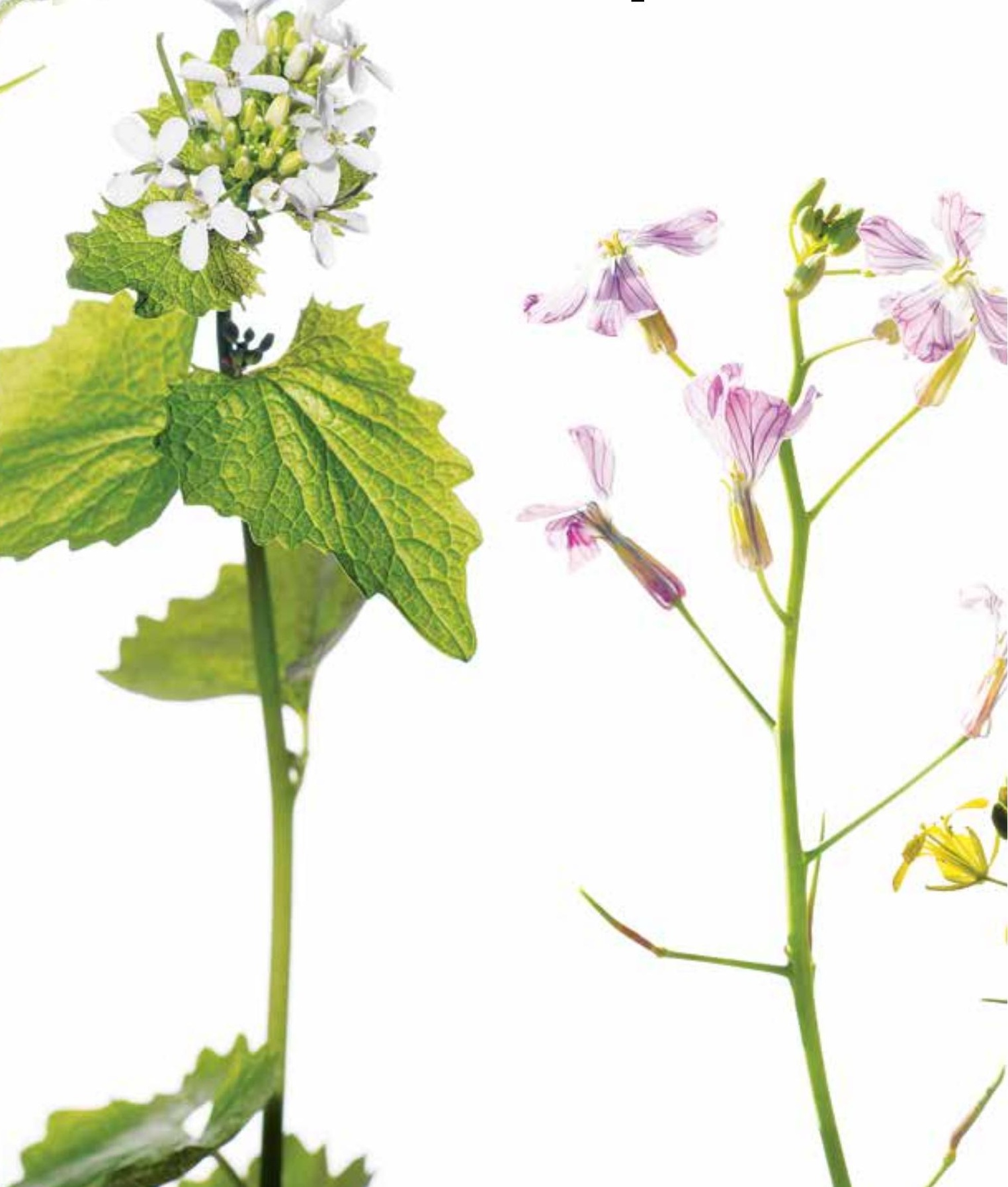


\section{4}

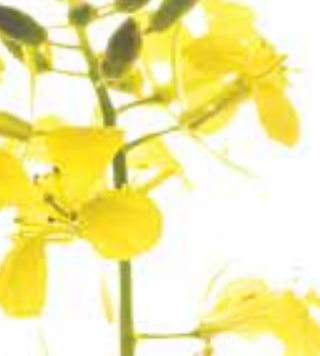

\section{Variation in herbivore-induced}

plant volatiles and alternative host plant cover mediate tritrophic interactions at the landscape scale

Yavanna Aartsma Yueyi Hao Marcel Dicke

Wopke van der Werf Erik H. Poelman Felix J.J.A. Bianchi 


\section{Abstract}

Tritrophic interactions may be affected both by local factors and by the broader landscape context. At small spatial scales of meters, parasitoid wasps that lay eggs in herbivorous caterpillars use herbivore-induced plant volatiles (HIPVs) to find hosts, but little is known about the possible effects of HIPVs at the landscape scale. It is not known whether HIPV emission by plants can enhance recruitment of parasitoids from the broader environment and increase rates of parasitism. Landscape-wide abundance of plant species providing hosts for parasitoid reproduction may be an important driver of parasitoid populations, influencing the pools of individuals from which herbivore-attacked plants may recruit parasitoids. Here, we studied in 19 landscapes whether parasitism of caterpillars of the butterfly Pieris brassicae on white cabbage was influenced by landscape composition and cover with Brassicaceous species that provide hosts for parasitoids of $P$. brassicae. We also investigated whether plant traits associated with HIPV-mediated attraction of parasitoids affected parasitism of $P$. brassicae caterpillars. Parasitism rates were higher on a more attractive cabbage accession than on a less attractive accession, but only when parasitism rates were high. Landscape-wide cover of brassicaceous plants and area of arable land were positively associated with parasitism rates. In contrast, forest area was negatively associated with parasitism rates, possibly because the dominant parasitoid species that parasitizes P. brassicae caterpillars, Cotesia glomerata, does not use forest as a habitat. The study shows that HIPV-mediated attractiveness to parasitoids increases parasitism when parasitoids are recruited from the broader landscape. Plant functional traits can be useful predictors of parasitism but need to be considered within the landscape context; this holds both for traits of the focal plant species on which parasitism is assessed and for traits of plant species that provide resources in the broader landscape. Accounting for species functional traits can thus improve insight in landscape effects on tri-trophic relationships underlying biological control of pests. 


\section{Introduction}

Species abundance and trophic interactions can be influenced by processes that extend beyond the local scale of a single patch or habitat (Thies et al., 2003; Tscharntke \& Brandl, 2004). For example, host-parasitoid dynamics can be influenced by characteristics of the landscape in which these interactions take place (Cronin \& Reeve, 2005). Parasitoids can be important natural enemies of pest insects in agriculture, but rely on resources, such as overwintering sites, floral resources or alternative hosts, that may be scattered across the landscape (Bianchi et al. 2006). There is a mature body of studies that focus on hostparasitoid dynamics in agricultural landscapes (Cronin \& Reeve, 2005; Bianchi et al., 2006; Chaplin-Kramer et al., 2011; Chaplin-Kramer et al., 2013, Karp et al., 2018), which indicates that parasitoid abundance and biological control of pest populations in agro-ecosystems are influenced by a wide range of landscape features such as crop/non-crop area (Rusch et al., 2010; Chaplin-Kramer et al., 2013), fragmentation/connectivity (Tscharntke \& Brandl, 2004), landscape management (Rusch et al., 2010), and landscape complexity (Poveda et al., 2012; Rusch et al., 2016).

Many studies on landscape composition use land use classes to explain parasitoid abundances. For example, non-crop area such as forests and non-woody seminatural habitats can be positively associated with parasitism rates (Thies et al., 2003; Costamagna et al., 2004; Bianchi et al., 2008b), and arable land can be negatively associated with parasitism rates in agricultural fields (Chaplin-Kramer et al., 2011; Poveda et al., 2012). However, land use classes are not a good predictor of parasitoid abundance when they do not capture the functionality of the habitats, for instance in terms of resource distribution for parasitoids and their hosts (Tscharntke et al., 2016; Karp et al., in press). In these cases a functional land cover approach is required to assess habitat suitability in more detail (Fahrig et al., 2010; Bianchi et al., 2012). For example, the abundance of wild plants that support host plants for the hosts of parasitoids might be a better predictor for parasitoid abundance than a general land use class "non-crop habitat" that may comprise a wide range of habitats with varying suitability for the parasitoid species (Crist et al., 2006; Isaacs et al., 2008; Bianchi et al., 2012). Especially for 
specialist parasitoids with a low dispersal capacity, presence of plant patches with hosts may be important for colonizing new areas (Elzinga et al., 2007).

There has been limited attention for the ways in which local-scale ecological processes interact with landscape scale processes to explain arthropod movement and their redistribution (Kremen, 2005; Schellhorn et al., 2014). Population redistribution processes can be understood in terms of patch leaving, interpatch movement and patch finding behaviour. These processes can be influenced by a myriad of factors, including habitat characteristics, motion and navigation capacity, perceptual range and environmental conditions (Schellhorn et al., 2014). Parasitoids use herbivore-induced plant volatiles (HIPVs) as cues to find their hosts (Vet \& Dicke, 1992; Hare, 2011). Herbivore-induced plant volatiles are emitted upon damage by herbivores and spread through space, where -together with volatiles from other plants- they may form a spatially heterogeneous volatile mosaic that parasitoids use to locate hosts (Aartsma et al., 2017). However, little is known about the spatial range at which HIPVs influence parasitoid foraging behaviour, especially at larger scales such as the landscape scale (Aartsma et al., 2017). Furthermore, there can be considerable variation in HIPV emission from plants, which may affect the distance from which they can be perceived (Rasmann et al., 2005; Poelman et al., 2009b; Mumm \& Dicke, 2010; Aartsma et al., 2018). The effect of HIPVs on parasitoid recruitment and parasitism of herbivorous insects has, to the best of our knowledge, not been studied at the landscape scale. This leaves the question unanswered how plant varieties with different attractiveness to parasitoids as a result of differences in HIPV emission moderate parasitism in different landscape settings.

In this study we quantified parasitism rates of P. brassicae caterpillars on two white cabbage accessions with different attractiveness to the parasitoid $C$. glomerata as a result of differences in HIPV blends. The study was conducted in 19 landscapes, without release of parasitoids, so that these had to be recruited from the surrounding landscape. The landscapes varied in the abundance of Brassicaceae species that might act as a source of parasitoids. We hypothesized that Brassicaceae cover is a meaningful predictor of parasitism rates of $P$. brassicae, and that a plant accession, which is more attractive to parasitoids has 
higher parasitism rates than a less attractive accession, independent of landscape composition. We also hypothesized that the area of arable land and intensively managed pastures is negatively associated with parasitism rates on the premise that these do not fulfil resource requirements of C.glomerata, and that the area of forest and non-woody semi-natural area are positively associated with parasitism rates, on the premise that wild Brassicaceae are important sources of C. glomerata and would occur more in non-crop habitats.

\section{Materials and Methods}

\section{Plants and insects}

Seeds of white cabbage (Brassica oleracea var alba) accessions Badger Shipper and Christmas Drumhead were obtained from the Centre for Genetic Resources (CGN-Wageningen, the Netherlands). In previous work, the accession Christmas Drumhead was found to be more attractive to the parasitoid C. glomerata than Badger Shipper, both under laboratory and field conditions (Poelman et al., 2009b; Aartsma et al., 2018). Plants were grown in peat soil in a greenhouse at Unifarm, Wageningen (L16:D8, 18-26 ${ }^{\circ} \mathrm{C}$ and $\left.40-70 \% \mathrm{RH}\right)$. Potted plants of six weeks old were used in the experiment.

Pieris brassicae caterpillars were reared in a greenhouse compartment $\left(20-22{ }^{\circ} \mathrm{C}\right.$ and $50-70 \% \mathrm{RH}$ ) on Brussels sprouts plants and first (L1) and second instar (L2) caterpillars were used in the experiment.

\section{Landscape selection}

We selected 19 landscapes in the vicinity of Wageningen, the Netherlands (Fig. 1). Landscapes were selected on the basis of the expected cover of brassicaceous plants in the area. The landscapes included a variety of land use types to reflect variation in landscape composition that is typical of the area (arable land, pastures, forest, and non-woody semi-natural areas). In the case of arable land, we chose mainly organic farm locations to minimize interference from pesticides. The minimum distance between the centres of two landscapes was at least $2 \mathrm{~km}$ (Fig. 1). 


\section{Field experiment}

To examine parasitism rates of $P$. brassicae caterpillars on cabbage plants of the accessions Badger Shipper and Christmas Drumhead, we performed a series of field experiments from May-August 2016. In each of the 19 locations, we placed potted cabbage plants near the centre of the landscape. The plants were placed in two small patches of four potted plants, one patch with the accession Badger Shipper and the other with the accession Christmas Drumhead. Both patches were positioned $25 \mathrm{~m}$ from the centre of the landscape and there was $50 \mathrm{~m}$ between the two patches (Fig 2A). The four plants were arranged in a square, with leaves touching. The two patches in each landscape were placed in a similar

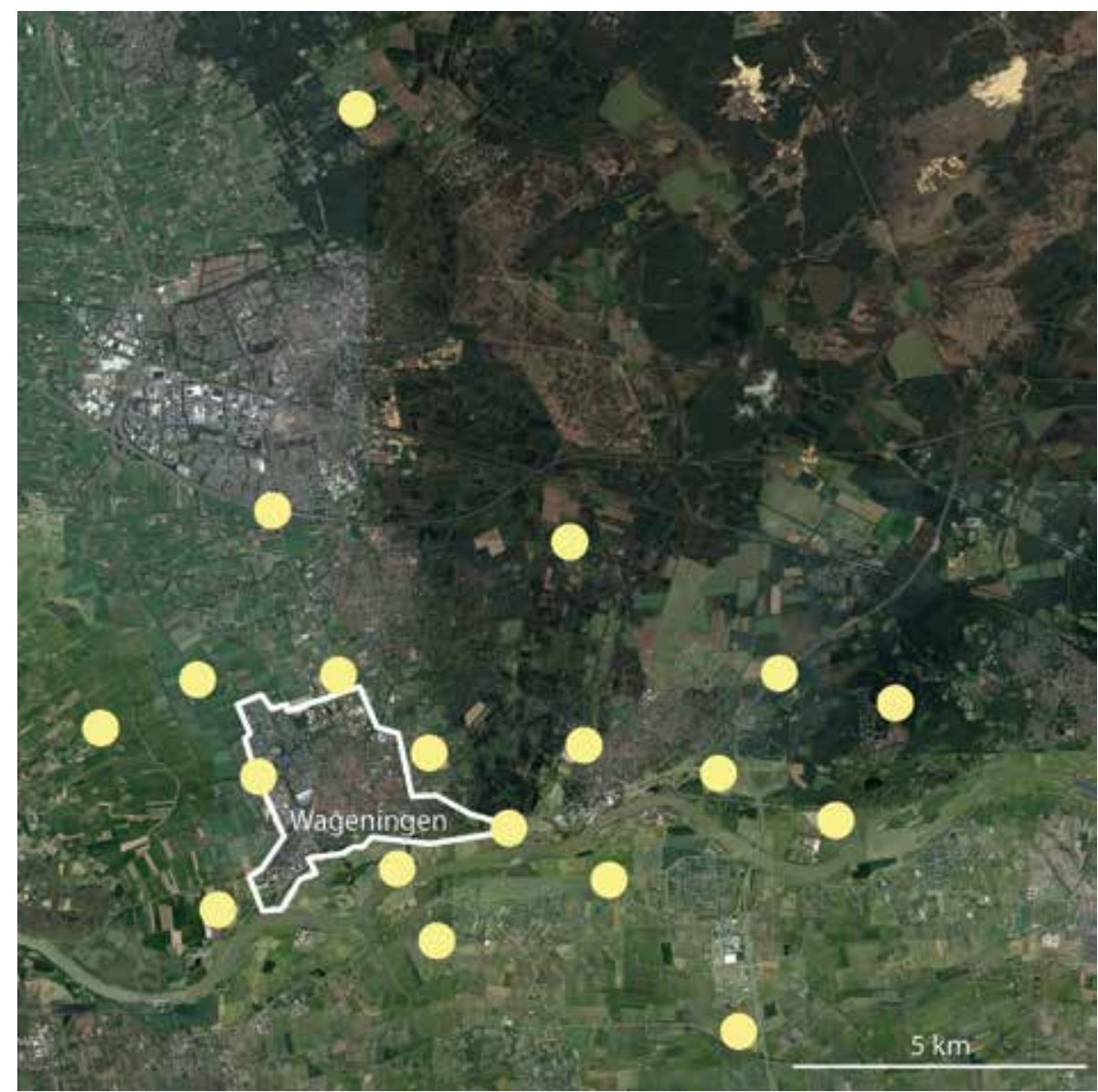

Figure 1: Locations of the 19 landscapes (yellow dots) around Wageningen, the Netherlands (white outline). Satellite image from Google Earth Pro. 
background vegetation. The plants were each inoculated with ten $P$. brassicae caterpillars per plant (40 in total in the patch) and were surrounded by a metal wire fence to prevent damage by vertebrate herbivores such as rabbits, hare and deer.

Four days after placing the plants in the landscape, the plants (and the remaining caterpillars) were recollected by cutting the stem and placing plants individually in labelled plastic bags. Plastic bags with plants were stored in a cold room (4 ${ }^{\circ} \mathrm{C}$ ), and during the days after recollecting the plants we dissected the caterpillars to assess whether they had been parasitized. In five cases, no caterpillars were recollected from a patch, resulting in five missing observations. Fewer caterpillars were recollected from patches with excessive slug damage.

The experiment was replicated five times over the season, in weeks 20, 22, 27, 29 and 34 . Between replicates the location of the two accessions in each landscape was swapped to minimize local vegetation background effects, but locations of the patches remained the same over the season. Placement of the plants and recollection four days later was conducted in two days (nine or ten locations per day).

\section{Quantification of landscape variables}

Land use data were extracted from the TOP10NL database. The vector-based TOP10NL database (PDOK, 2016) was used in ArcGIS to assess the area of arable land, pasture, forest and non-woody semi-natural habitat within circles of 100, 200, 300 and $1000 \mathrm{~m}$ radius around the centre of each landscape. The map information from TOP10NL was checked by ground-truthing and adjusted when needed.

We used brassicaceous plant cover as a measure of their relative abundance. By estimating the proportion cover by Brassicaceae in a field, we take into account the size of the plants rather than the number of individual plants. Brassicaceous plant cover was assessed in a radius of $300 \mathrm{~m}$ around the centre of each landscape, once between week 24 and 27 of 2016, which coincides with the flowering time of many Brassicaceae species. The brassicaceous plant cover was 
assessed in each landscape element (e.g. arable field, pasture, forest patch) by randomly selecting three locations in the interior and three at the border of the element (directly at the interface of the adjoining element), and estimating the percentage cover of brassicaceous plants in a $1 \mathrm{~m}^{2}$ quadrat (Fig. 2). Edges and interiors were sampled separately because the density of brassicaceous plants may differ. The most common brassicaceous species were cultivated Brassica oleracea, and the wild plants Brassica nigra, Brassica rapa, Alliaria petiolata, Raphanus spp., Capsella bursa-pastoris and Sinapis spp.

Recorded Brassicaceae cover data from quadrats were converted to Brassicaceae cover in $\mathrm{m}^{2}$ for each landscape element by multiplying the mean brassicaceous plant cover per element section (border or interior) with the area $\left(\mathrm{m}^{2}\right)$ of each element section. For borders we multiplied the length of the border with a width of the quadrat $(1 \mathrm{~m})$ to obtain the area of the border. Brassicaceae covers for all elements were summed to obtain Brassicaceae cover estimates in 100, 200 and $300 \mathrm{~m}$ radius landscape sectors.

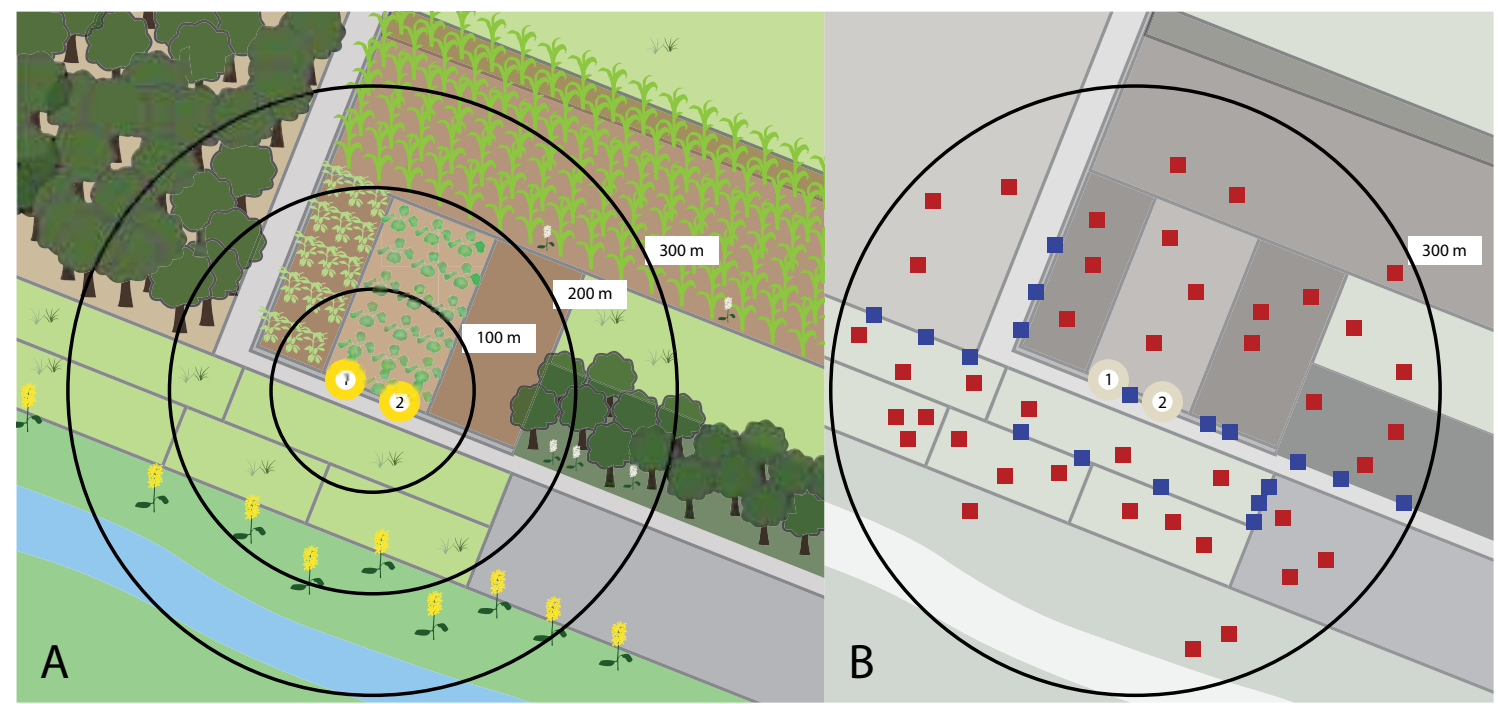

Figure 2: A) hypothetical landscape with the location of the experimental cabbage patches (white circles with yellow border, location 1 and 2 for two cabbage accessions differing in HIPV profile) and the $100 \mathrm{~m}$, $200 \mathrm{~m}$ and $300 \mathrm{~m}$ radius circles surrounding the patches (1000 m circle not shown). B) The landscape was subdivided into different elements and brassicaceous plant cover was assessed in each element by three quadrant observations in the interior (red squares) and three in the border (blue squares). 


\section{Data analysis}

To investigate whether cabbage accession and landscape variables affect parasitism rates in patches across landscapes, data on parasitism rates were analysed using a generalized linear mixed model with logit link. The data of the four plants in each patch were pooled and the number of parasitized caterpillars out of the total number of recollected caterpillars was analysed as a binomially distributed response variable. We did not discriminate between different parasitoid species in the analysis.

Table 1: Overview of descriptive statistics of brassicaceous plant cover in landscape elements in $300 \mathrm{~m}$ landscape circles in 19 landscapes. Columns represent total area (ha), number of examined elements (N), number of elements with brassicaceous plant species ( $\mathrm{N}$ Brassicaceae), average proportion Brassicaceae cover \pm SEM (dimensionless) in element interiors (Cover interior), average proportion Brassicaceae cover \pm SEM (dimensionless) in element borders (Cover border) and the overall area-weighted proportion of interior and border combined (Cover combined). The land use type 'Other' includes road verges, urban areas and some miscellaneous elements occurring only once in the 19 landscapes. *mixed crops consisted of vegetable gardens or small-scale strip cropping.

\begin{tabular}{|c|c|c|c|c|c|c|c|}
\hline Land use ty & & $\begin{array}{l}\text { Area } \\
\text { (ha) }\end{array}$ & $N$ & $\begin{array}{l}\text { N } \\
\text { Brassicaceae }\end{array}$ & $\begin{array}{l}\text { Cover interior } \\
\text { (proportion) }\end{array}$ & $\begin{array}{l}\text { Cover border } \\
\text { (proportion) }\end{array}$ & $\begin{array}{l}\text { Cover combined } \\
\text { (proportion) }\end{array}$ \\
\hline Pastures & & 01120 & 120 & 0 & $4.8 \times 10^{-5}$ & $4.0 \times 10^{-4}$ & $6.3 \times 10^{-5}$ \\
\hline & & 212.8 & 100 & 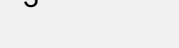 & $\pm 4.8 \times 10^{-5}$ & $\pm 6.0 \times 10^{-5}$ & $\pm 6.0 \times 10^{-5}$ \\
\hline Arable & Total & 120.6 & 73 & 21 & $0.071 \pm 0.023$ & $0.067 \pm 0.022$ & $0.071 \pm 0.023$ \\
\hline & maize & 43.9 & 20 & 1 & $0.012 \pm 0.012$ & $0.007 \pm 0.007$ & $0.012 \pm 0.012$ \\
\hline & wheat & 37.1 & 16 & 1 & 0 & $0.003 \pm 0.003$ & $5.3 \times 10^{-6} \pm 5.3 \times 10^{-6}$ \\
\hline & Brassicaceae & 11.5 & 10 & 10 & $0.44 \pm 0.119$ & $0.412 \pm 0.126$ & $0.438 \pm 0.12$ \\
\hline & mixed crops* & 8.4 & 7 & 5 & $0.055 \pm 0.018$ & $0.055 \pm 0.018$ & $0.055 \pm 0.018$ \\
\hline & carrots & 7.3 & 1 & 0 & 0 & 0 & 0 \\
\hline & fallow & 5.0 & 8 & 1 & $0.013 \pm 0.013$ & $0.013 \pm 0.013$ & $0.013 \pm 0.013$ \\
\hline & potatoes & 3.4 & 6 & 1 & 0 & $0.003 \pm 0.003$ & $8.6 \times 10^{-5} \pm 8.6 \times 10^{-5}$ \\
\hline & onions & 1.6 & 1 & 0 & 0 & 0 & 0 \\
\hline & lettuce & 1.5 & 1 & 0 & 0 & 0 & 0 \\
\hline & flower strip & 1.0 & 2 & 2 & $0.205 \pm 0.195$ & $0.205 \pm 0.195$ & $0.205 \pm 0.195$ \\
\hline Forest & & 66.4 & 57 & 7 & $\begin{array}{l}5.8 \times 10^{-5} \\
\pm 5.8 \times 10^{-5}\end{array}$ & $\begin{array}{l}0.007 \\
\pm 0.003\end{array}$ & $\begin{array}{l}2.2 \times 10^{-4} \\
\pm 1.19 \times 10^{-4}\end{array}$ \\
\hline Non-wood & semi-natural & 9.5 & 7 & 2 & $0.007 \pm 0.007$ & $0.057 \pm 0.056$ & $0.007 \pm 0.007$ \\
\hline Other & & 70.5 & 53 & 11 & $0.02 \pm 0.007$ & $0.023 \pm 0.009$ & $0.02 \pm 0.007$ \\
\hline
\end{tabular}


We first constructed models using only structural landscape variables (i.e. area of arable land, pasture, forest, and non-woody semi-natural habitat, and accession (Christmas Drumhead vs. Badger Shipper) as fixed factors. Trial nested within landscape and the location of the patch within a landscape (on which the accessions were switched between trials) were included as random factors. We used the Akaike Information Criterion corrected for the number of data (AICc) to determine the model structure for the random effects (Zuur et al., 2009). We determined which fixed factors were most important for the model by using the 'dredge' model selection procedure, which calculates all possible factor combinations and sorts the models according to the value of AICc. For the selected models with the lowest AICc values, we also calculated the more conservative Bayesian Information Criterion (BIC). The marginal $\mathrm{R}^{2}$ was calculated to evaluate the explained variance of the fixed effects (Nakagawa \& Schielzeth, 2012). This analysis was conducted separately for spatial scales of $100 \mathrm{~m}, 200 \mathrm{~m}, 300 \mathrm{~m}$ and $1000 \mathrm{~m}$ radius.

In a second analysis, we used a functional landscape variable, i.e. brassicaceous plant cover, which captures information on the alternative host plant cover in the landscape, and accession (treatment) as fixed factors. Brassicaceae cover was calculated in $\mathrm{m}^{2}$ by multiplying the proportion cover in landscape elements by the area of the same elements and adding them up for the complete landscape. Brassicaceae cover was double square-root transformed to meet linearity criteria. The same structure for random effects was used as in the analysis using land use classes. All analyses were performed in R and the packages lme4, MuMIn (Barton, 2016; Bates et al., 2015). Plots were made in ggplot2 and model output tables with the package stargazer (Wickham, 2009; Hlavac, 2018).

\section{Results}

Across the five replicates of the experiment, we recovered 3302 out of 7400 caterpillars ( $44 \% \pm 21.9 \%$ per patch; mean $\pm S D$ ) placed on plant patches in the 19 landscapes. The overall average parasitism rate of the recovered caterpillars was $20 \pm 35 \%$ (mean $\pm S D$ ), varying from $6.6 \%$ to $34 \%$ between replicates. 
Cotesia glomerata was responsible for $98 \%$ of the parasitism events, while Cotesia rubecula and tachinid flies were responsible for the remaining $2 \%$. The tachinid fly larvae were found in caterpillars which also had C. glomerata eggs.

The first analysis, considering structural land use variables, indicated that parasitism rates were significantly negatively associated with forest at scales of $100 \mathrm{~m}, 200 \mathrm{~m}$ and $300 \mathrm{~m}$, but not at $1000 \mathrm{~m}$ (Fig. 3B, Table 2). Parasitism rates were also negatively related to non-woody semi-natural habitat, but this was only significant at $200 \mathrm{~m}$. There was a significant interaction between arable land and cabbage accession at all scales, indicating that parasitism rates were higher on Christmas Drumhead than on Badger Shipper when the area of arable land was high, whereas there was no significant difference between the accessions when the area of arable land was small (Fig. 3A, Table 2). While the interaction between accession and area of arable land was also significant at a scale of 1 $\mathrm{km}$, parasitism rates were not strongly influenced by the area of arable land and the largest differences between parasitism rates on the two cultivars were found in landscapes with a relatively low proportion of arable land (Supplemental Information Fig. 1, Table 2).

In the second analysis with the functional variable brassicaceous plant cover, parasitism rates were significantly influenced by brassicaceous plant cover, cabbage accession and their interaction (Table 3). In landscapes where cover by Brassicaceae was low, parasitism rates were low on both accessions. Parasitism rates increased with higher brassicaceous plant cover, and parasitism rates were higher on the attractive accession Christmas Drumhead than on the less attractive Badger Shipper (Table 3, Fig. 4).

There was a strong positive correlation between area of arable land and Brassicaceae cover $(r=0.79, p<0.001)$. When comparing the first analysis with the second, the analysis with land use variables had a lower AICc than the model with the functional variable Brassicaceae cover at the $100 \mathrm{~m}$ scale, but at $200 \mathrm{~m}$ and $300 \mathrm{~m}$ AICc's were similar (Supplemental Fig. 2). Using the more conservative Bayesian Information Criterion, the analysis with land use variables had a lower $\mathrm{BIC}$ as the model with the functional variable Brassicaceae 
Chapter 6
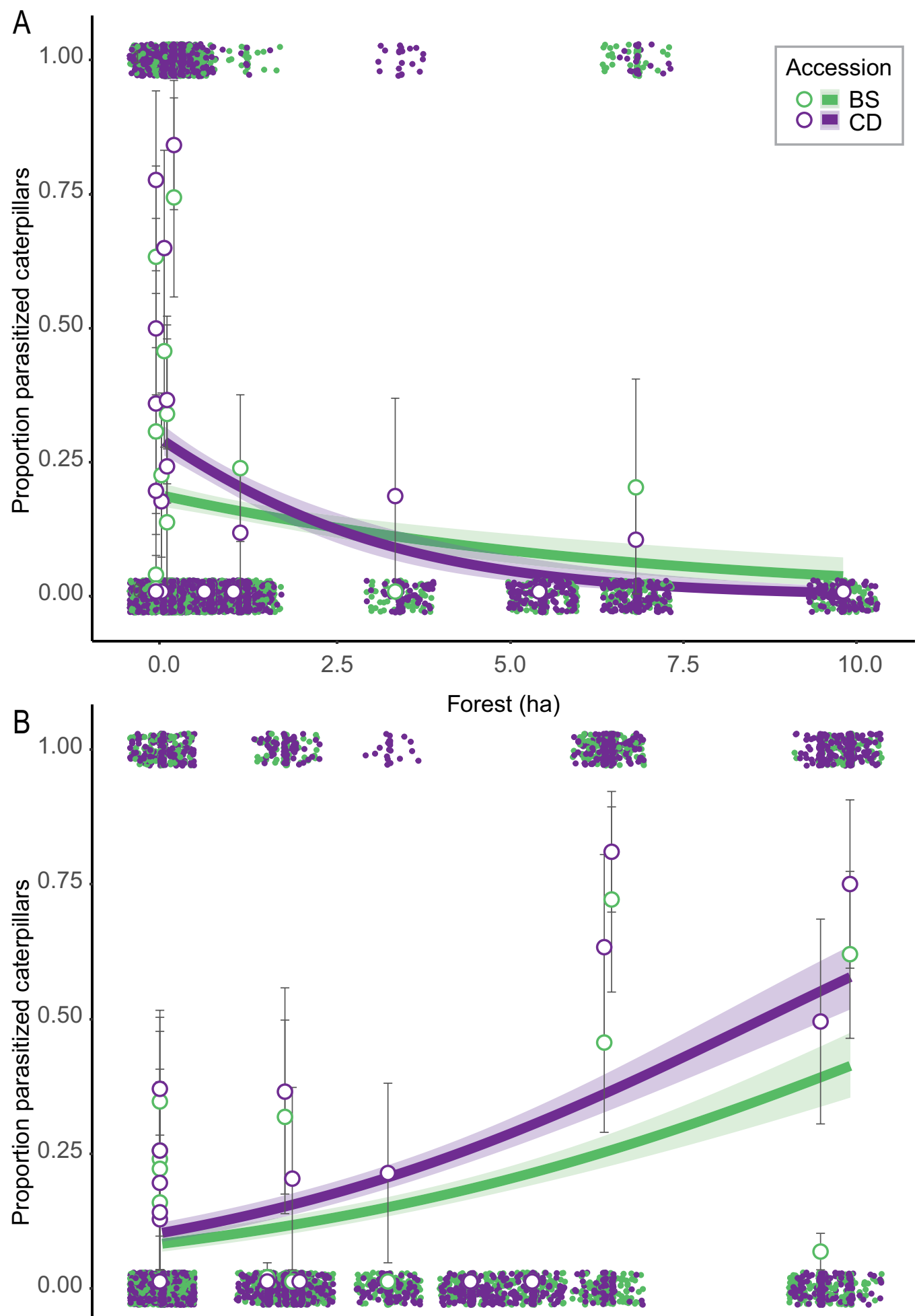
Figure 3 (left): Parasitism rates on the white cabbage accessions Badger Shipper (BS; green) and Christmas Drumhead (CD; purple) in landscapes varying in area of forest and area (A) of arable land (B) in a $200 \mathrm{~m}$ radius circle surrounding the patches. Open circle markers show averages across five temporal replicates and error bars reflect SEM. Binary responses of individual larvae are presented as a jitter plots at 0 (no parasitism) and 1 (parasitism). At a scale of $200 \mathrm{~m}$, arable land area and area of forest were the significant variables selected in the structural land use model (Table 2).

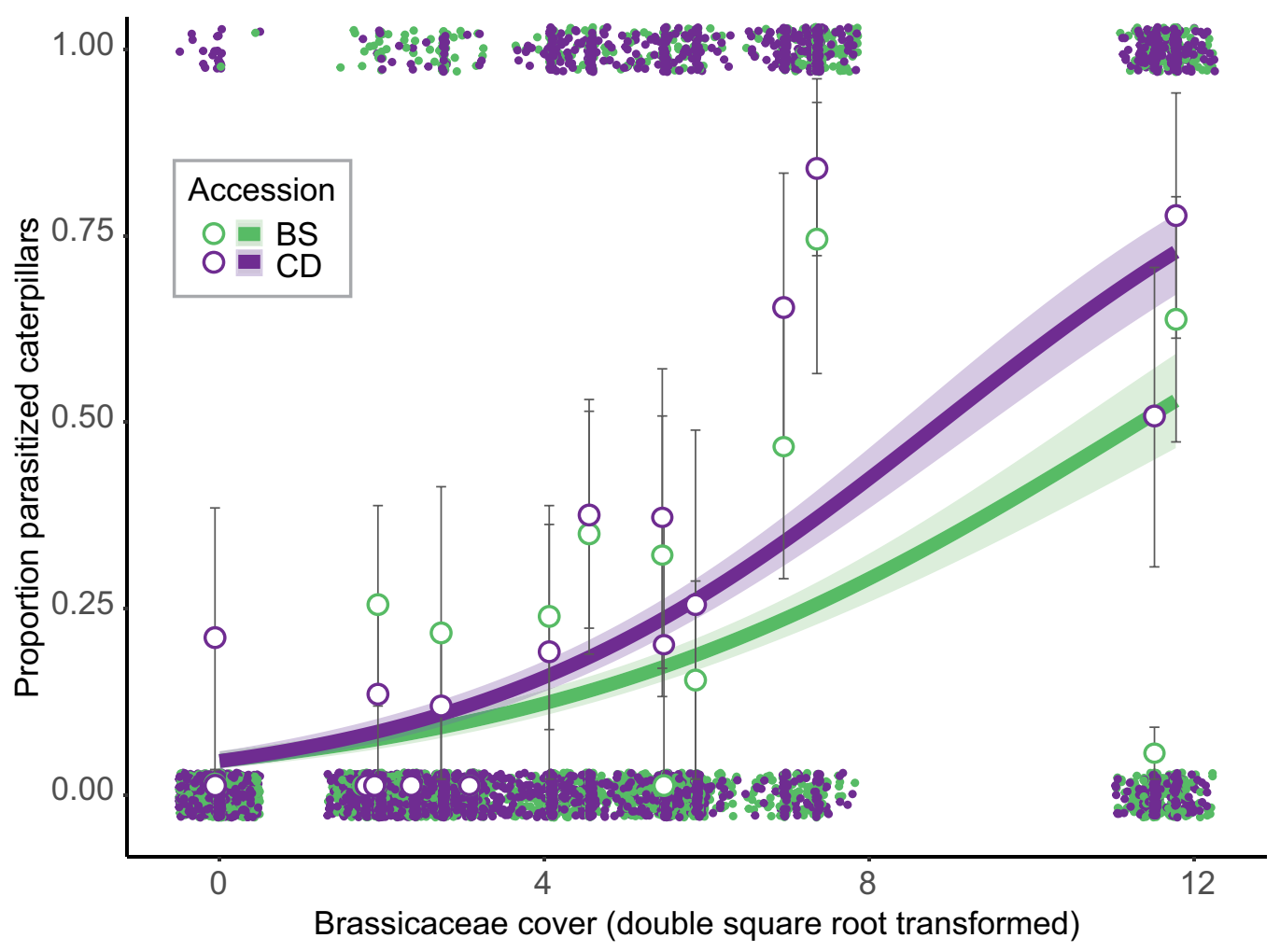

Figure 4: Relationship between parasitism rates on the white cabbage accessions Badger Shipper (BS; green) and Christmas Drumhead (CD; purple) and coverage by brassicaceous plants within a radius of $200 \mathrm{~m}$ from the experimental patches. Open circle markers show averages across five temporal replicates and error bars reflect SEM. Binary responses of individual larvae are presented as a jitter plots at 0 (no parasitism) and 1 (parasitism). Brassicaceous plant cover was transformed as where $x$ is the measured cover fraction in the landscape circle. Brassicaceae cover was calculated by multiplying the proportion cover in landscape elements by the area of the respective elements and summing them up for the complete landscape sector. 
Table 2: Overview of explanatory variables and estimated coefficients of the selected most parsimonious models for parasitism rate (number of parasitized caterpillars/total number of caterpillars recovered from patch) at four different spatial scales, when using structural land use variables and accession of the focal plants (more or less attractive via HIPV emission) as predictors. A slash symbol (/) indicates that this variable was not selected at this spatial scale. The reference treatment for accession was Badger Shipper. Bold numbers indicate significant differences.

Response variable: Parasitized caterpillars/total recollected caterpillars

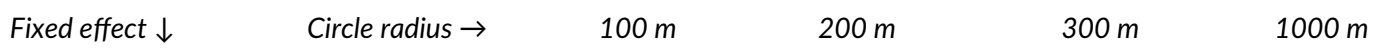

\begin{tabular}{|c|c|c|c|c|c|}
\hline \multirow[t]{2}{*}{ Accession } & Badger Shipper & - & - & - & - \\
\hline & Christmas Drumhead & $0.328(0.320)$ & $0.236(0.335)$ & $-0.030(0.376)$ & $-0.694(0.537)$ \\
\hline \multicolumn{2}{|l|}{ Arable (ha) } & $-0.590(1.428)$ & $-0.278(0.367)$ & $0.054(0.160)$ & $-0.047(0.034)$ \\
\hline \multicolumn{2}{|l|}{ Forest (ha) } & $-6.152^{*}(2.478)$ & $-2.200^{* *}(0.799)$ & $-0.556^{*}(0.277)$ & $-0.032(0.022)$ \\
\hline \multicolumn{2}{|c|}{ Pastures (ha) } & $-3.439(2.508)$ & - $1.191(0.642)$ & / & / \\
\hline \multicolumn{2}{|c|}{ Semi -natural (ha) } & $-4.842(2.495)$ & $-1.802^{*}(0.803)$ & $-0.320(0.252)$ & $-0.078(0.057)$ \\
\hline \multicolumn{2}{|c|}{ Accession $\times$ Arable (ha) } & $0.968^{* * *}(0.197)$ & $0.281^{* * *}(0.056)$ & $0.159^{* * *}(0.032)$ & $0.058^{* * *}(0.013)$ \\
\hline \multicolumn{2}{|l|}{ Constant } & $1.112(5.361)$ & 3.275 (5.187) & $-5.780^{* * *}(1.693)$ & $-4.614^{*}(2.087)$ \\
\hline \multicolumn{2}{|l|}{$\mathrm{AICc}$} & 601.69 & 604.43 & 608.55 & 612.41 \\
\hline
\end{tabular}

Table 3: Overview of explanatory variables and estimated coefficients of the selected most parsimonious models for parasitism rate (number of parasitized caterpillars/total number of caterpillars recovered from patch) at four different spatial scales, when using brassicaceous plant cover and accession of the focal plants (more or less attractive via HIPV emission) as predictors.

Response variable: Parasitized caterpillars/total recollected caterpillars

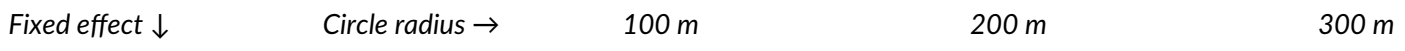

Accession Badger Shipper

Christmas Drumhead $\quad 0.787(0.674)$

$\begin{array}{lr}-1.226^{*}(0.599) & -1.201^{*}(0.604) \\ 0.955^{* *}(0.332) & 0.873^{* *}(0.302) \\ 0.386^{* * *}(0.082) & 0.343^{* * *}(0.075) \\ -10.225^{* * *}(1.518) & -10.144^{* * *}(1.495) \\ 605.3 & 605.2\end{array}$

$\mathrm{AICC}$

$1.191^{* *}(0.421)$

$0.422^{* * *}(0.120)$

Brassicaceae cover $\times$ Christmas Drumhead

$-10.129^{* * *}(1.472)$

614.2

$$
{ }^{*} \mathrm{p}<0.05 ; \quad{ }^{* *} \mathrm{p}<0.01,{ }^{* * *} \mathrm{p}<0.001
$$


cover at the $100 \mathrm{~m}$ scale, but higher BIC values at $200 \mathrm{~m}$ and $300 \mathrm{~m}$, indicating that the model with the functional variable Brassicaceae cover received more support from the data than the model with land use variables at $200 \mathrm{~m}$ and 300 $\mathrm{m}$ (Supplemental Fig. 2). Marginal $\mathrm{R}^{2}$ for both models were similar for $100 \mathrm{~m}$ and $200 \mathrm{~m}$, while the land use models contain more variables, but was higher for the model with the functional variable than the model with land use variables (Supplemental Fig. 2).

\section{Discussion}

In this study we show that parasitism rates of $P$. brassicae caterpillars correlate with landscape scale and local scale factors. We report three key findings. First, parasitism rates of caterpillars feeding on the more attractive accession, Christmas Drumhead, were higher than on the less attractive accession, Badger Shipper, but this was only the case in landscapes with relatively high parasitism rates. Second, parasitism rates were positively associated with the cover of Brassicaceae plants and the area of arable land, and negatively associated with forest and non-woody semi-natural habitat. Third, the functional landscape variable brassicaceous plant cover was strongly and positively correlated with the structural landscape variable proportion of arable land.

Parasitism rates were influenced by the relative attractiveness of the cabbage accession, but only when parasitism rates were relatively high. This confirms findings of Poelman et al. (2009b) who showed that the accession Christmas Drumhead is more attractive than Badger Shipper in laboratory and small-scale field experiments. Here we show that these accessions can explain variation in parasitism rates at the landscape scale. Since parasitism rates were positively associated with Brassicaceae cover and proportion of arable land, this suggests that landscapes with a high Brassicaceae cover and proportion of arable land supported higher parasitoid densities than landscapes with low Brassicaceae cover and proportion of arable land. There was no difference between the cabbage accessions when parasitism rates were low. This could be a consequence of the low numbers per se, which generally tends to make it difficult to find significant 
effects. When parasitoid densities are low, finding a herbivore-infested cabbage plant by a parasitoid resembles drawing from a binomial probability distribution with a low number of trials (e.g. flipping a coin just a few times). Even though the attractive accession may have a higher probability of being found (reflecting flipping a biased coin which has a higher probability of obtaining a head than tail) the likelihood of showing significant differences between cabbage accessions (or heads and tail) is limited as long as the number of trials is small. Only with a substantial number of trials significant differences among cabbage accessions are likely to show. This is exactly what we found in our experiment.

Higher cover of food plants for caterpillar hosts has earlier been linked to higher parasitoid densities, usually indirectly via host abundance (Costamagna et al., 2004; Petermann et al., 2010). Our results suggest that the difference in parasitism rates on Christmas Drumhead and Badger Shipper is probably not only due to choice behaviour associated with a preference for more attractive plants (i.e. preference for one accession when perceiving the odours of two accessions), but also the parasitoid's ability to locate the plant via HIPVs from further away. Previously, we found that the attractive accession Christmas Drumhead could attract parasitoids from a distance of $20 \mathrm{~m}$ in the field whereas the less attractive accession Badger Shipper showed such attraction at $10 \mathrm{~m}$ distance, but not at $20 \mathrm{~m}$ (Aartsma et al., 2018). Larger distance of attraction would result in a larger 'parasitoid catchment area' and may therefore explain higher parasitism rates on the attractive accession. We are not aware of other landscape scale studies on parasitism that accounted for a possible role of HIPVs (Schellhorn et al., 2014; Aartsma et al., 2017). The current results are therefore unique, suggesting that HIPV have consequences for natural biological control in a landscape context.

The positive association between parasitism rates in P. brassicae caterpillars and the proportion arable land does not seem to match with other landscape studies, which consider the area of arable land in a landscape as a measure of landscape simplicity or agricultural intensification. For instance, natural enemy abundance, including parasitoids, was negatively associated with arable land (Bianchi et al., 2008b; Poveda et al., 2012; Rusch et al., 2016; Tscharntke et al., 2005a, but see Vollhardt et al., 2008, Zhou et al., 2014). Furthermore, the negative association 
between parasitism rates and forest and non-woody semi-natural habitats in our study contrasts with previous work that reported positive associations (Bianchi et al., 2008; González et al., 2017). However, individual taxa may show contrasting responses to landscape complexity with some species showing positive and other species negative associations (Menalled et al., 2003). This difference might be explained by the life history of the species. For example, cereal aphid parasitoid diversity and density was similar for wheat fields in simple and complex landscapes, possibly because all resources including honeydew as a food source can be found in wheat fields (Vollhardt et al., 2008). In another study, one parasitoid species would mainly parasitize hosts in vineyards, while another species mainly parasitized hosts in non-crop area surrounding these vineyards (Feng et al., 2017). In more complex community settings, presence of Pieris rapae caterpillars reduced aphid parasitism in broccoli fields and increased aphid densities, showing that other community members can disrupt pest suppression (Blubaugh et al., 2018). In our specific study system of P. brassicae and their dominant parasitoid species, C. glomerata, life history of the parasitoid species might help explain variation in parasitism in different landscape settings. Brassicaceae cover in forests was rather low in our study area and consisted mostly of the species Allaria petiolata. While the main hosts of C. glomerata, P. brassicae and P. rapae, can oviposit on and feed from this plant species (Heinen et al., 2016), it may not be their preferred host plant in natural conditions. Brassicaceae cover was highest in arable land, in particular cabbage fields, which suggests that (organic) arable land contains suitable habitats for P. brassicae and $P$. rapae. Information on the herbivore community that plant species support in combination with their cover might improve predictions on parasitism rates. In addition, both butterfly and parasitoid might prefer open landscapes to wooded areas (Friberg et al., 2015). Therefore, it is important to not only examine broadscale effects of non-crop versus crop habitats, but to also take into account ecological habitat requirements of the species under study.

In our study, arable land coverage correlated with the functional landscape variable of cover of brassicaceous plants. Cultivated Brassicaceae are by definition a part of arable land and at the same time are planted in high densities compared to wild Brassicaceae. Therefore, locations with the highest Brassicaceae cover also had 
the most arable land. As these locations were organically grown brassicaceous crops, we do not expect strong negative effects of farm management practices (e.g. synthetic insecticide applications) on parasitoid populations in these locations. In conventional farms, pesticide use may negatively impact natural enemy abundance (Rusch et al., 2010). Arable land as a simple and easy to obtain land use class may not be a suitable predictor of parasitism rates in this specialised system. However, our field survey provided estimates of Brassicaceae cover in different crop types. These could be used to predict Brassicaceae cover when the crop composition of arable land is known and thereby be used as a proxy to determine parasitism rates.

\section{Application}

Studies on HIPVs there have paid considerable attention to the use of indirect defence via HIPVs or the use of plants which are better at attracting natural enemies to improve biological control in agricultural fields (Dicke et al., 1990; Cortesero et al., 2000; Kaplan, 2012a; Penaflor \& Bento, 2013; Stenberg et al., 2015; Turlings \& Erb, 2018). Similarly, there is attention to habitat management at the landscape scale to facilitate natural enemy abundance in agricultural fields and create pest-suppressive landscapes (Tscharntke et al., 2007; Bianchi \& Wäckers, 2008; Woltz et al., 2012; Wyckhuys et al., 2013; Tscharntke et al., 2016; Gurr et al., 2017). This study shows that although plant attractiveness via HIPVs can lead to higher parasitism rates in patches with a more attractive variety, landscape characteristics such as the area of arable land, forest and host plant cover are also important determinants of parasitism in the field. Therefore, for enhanced attraction of natural enemies through HIPVs in realistic field situations, it is important to consider the landscape composition surrounding crop fields and connectivity with populations of natural enemies. This means that the use of plant varieties with enhanced HIPV blends and habitat management practices are both crucial and interdependent when it comes to improving natural biological control in agricultural fields. It is also important to consider which natural enemies are preferred to be attracted to the field as biological control agents. Specialist natural enemies may require more specific habitat types, linked 
to their specific host plant species, rather than a general semi-natural area or forest. For generalist natural enemies, this may be less of a problem as they can switch prey when the pest species is not available in alternative habitat.

\section{Conclusion}

Structural land use variables are often used in landscape studies because they are simple to obtain and correlate to measurements of arthropod abundance. However, these structural variables do not necessarily capture the ecological prerequisites of arthropods that are needed for their survival and reproduction, such as overwintering sites, floral resources or alternative hosts. Relationships between structural land use variables are often only presumed or speculated on a posteriori basis. While structural land use variables may be useful for general measurements such as monitoring (functional) groups of arthropods or pest pressure/control in a landscape, for study systems comprising specialist insects it will be more useful to consider more biologically meaningful variables such as host-plant cover. Moreover, it is also important to consider how focal plant traits such as attractiveness to natural enemies can affect movement of natural enemies. Our study highlights the importance of integrating local scale processes driven by plant-trait variation with landscape scale processes that determine parasitoid abundance in our understanding of what determines the strength of tri-trophic interactions and to better understand and manage parasitism as a tool in biological pest control.

\section{Acknowledgements}

We thank Jasper van Ruijven for advice on setting up the experiment and Tim van Schelt for assisting during part of the field work. 


\section{Supplemental information}

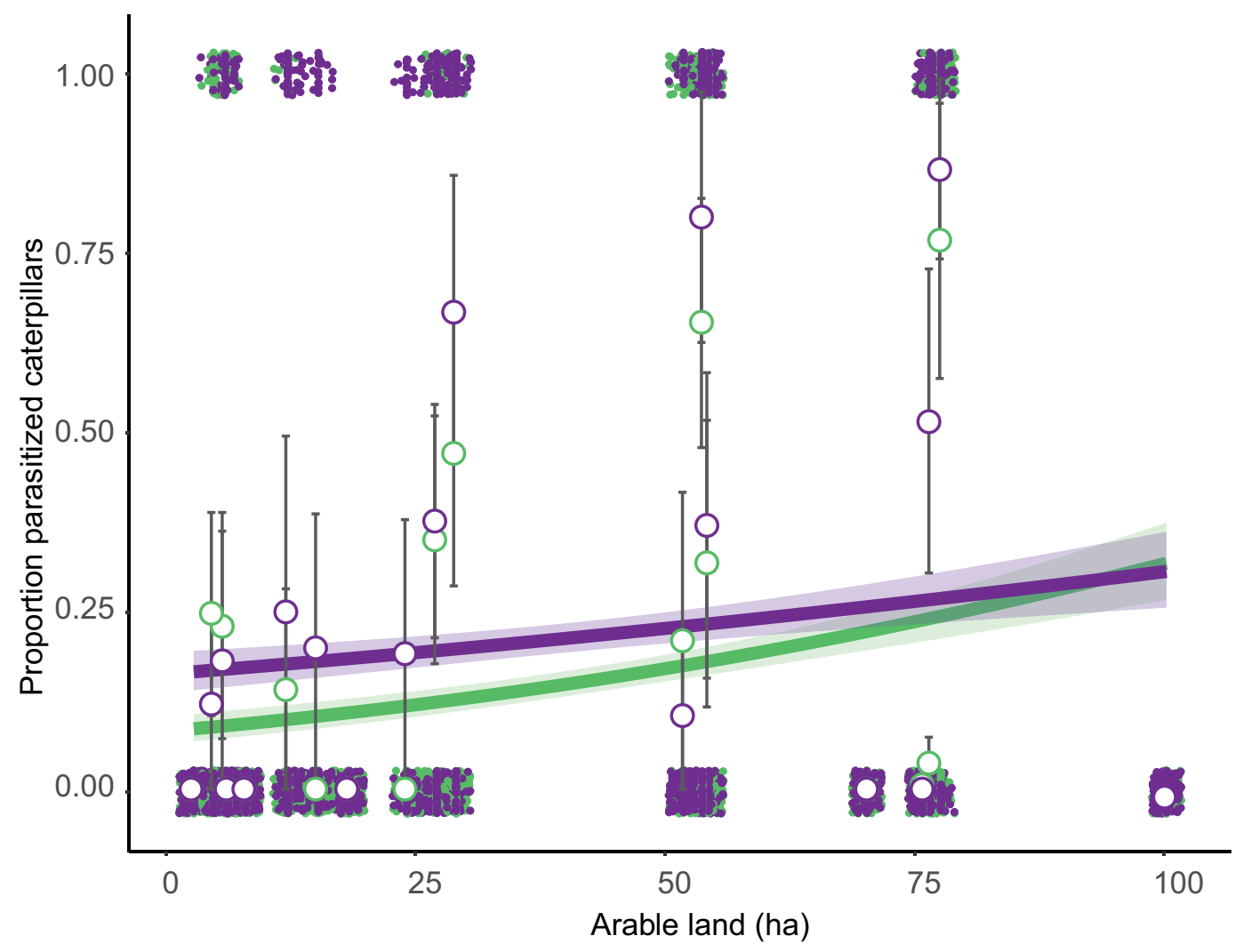

Supplemental Figure 1: Parasitism rates on different white cabbage accessions Badger Shipper (BS; green) and Christmas Drumhead (CD; purple) and in landscapes varying in area of arable land 1000 $\mathrm{m}$ radius circle surrounding the patches, the most important variable selected in the structural land use model. Open circle markers show averages across five temporal replicates and error bars reflect SEM. Binary responses of individual larvae are presented as a jitter plots at 0 (no parasitism) and 1 (parasitism). 


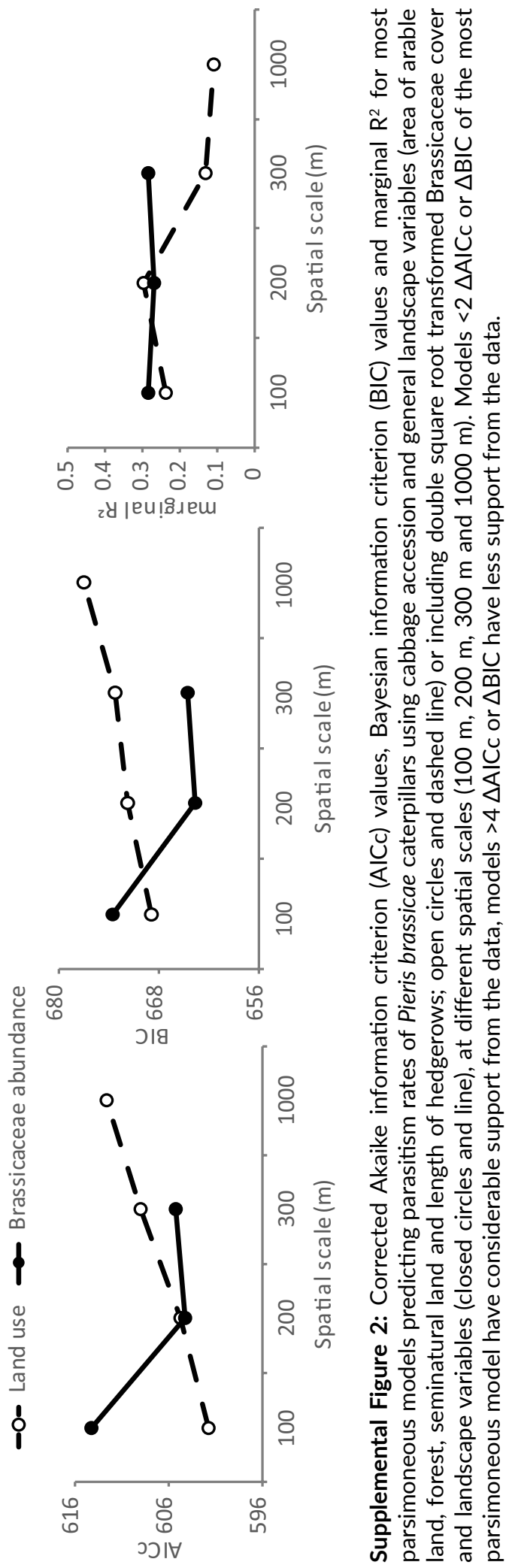




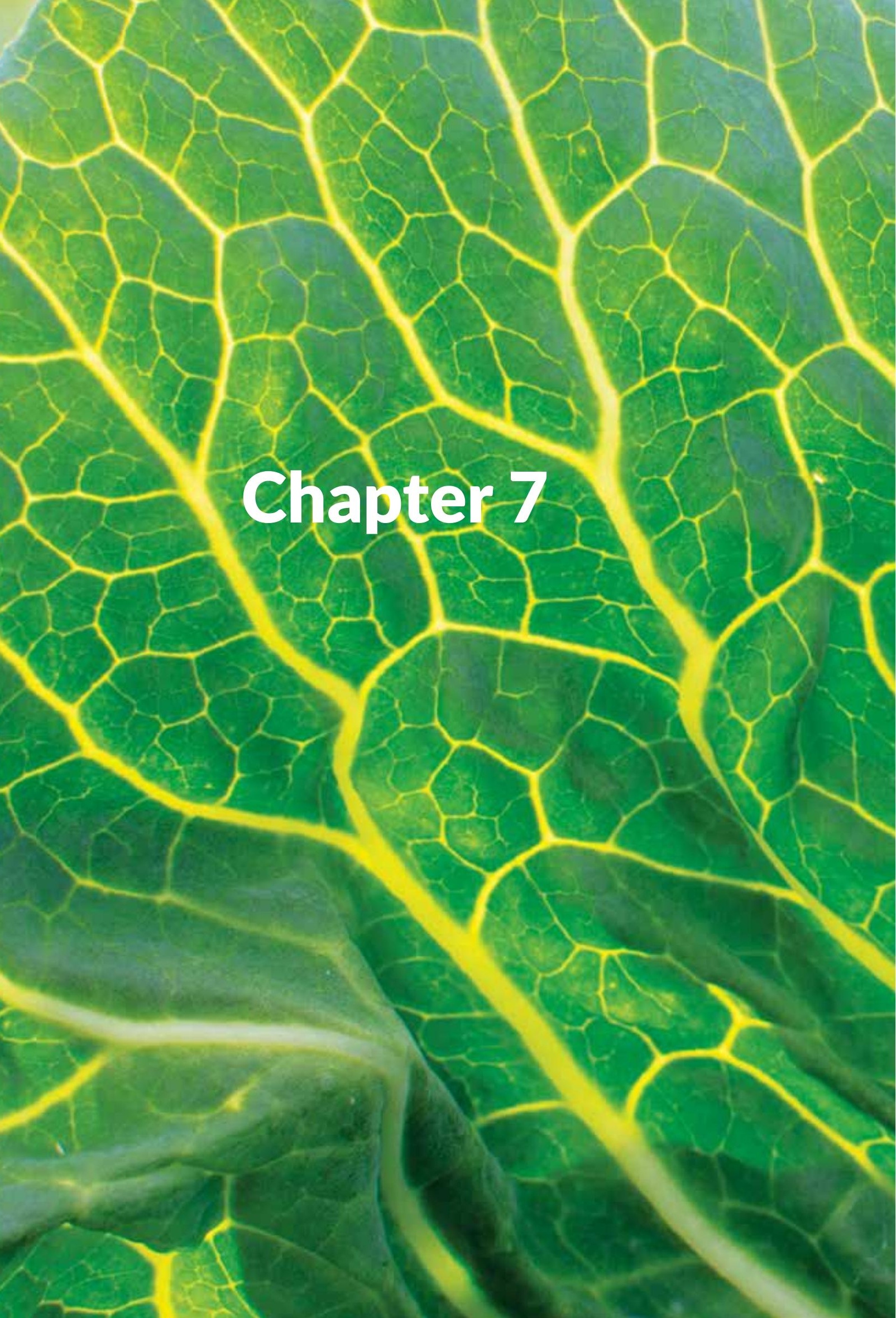




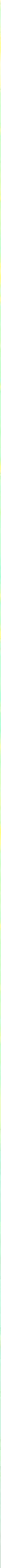



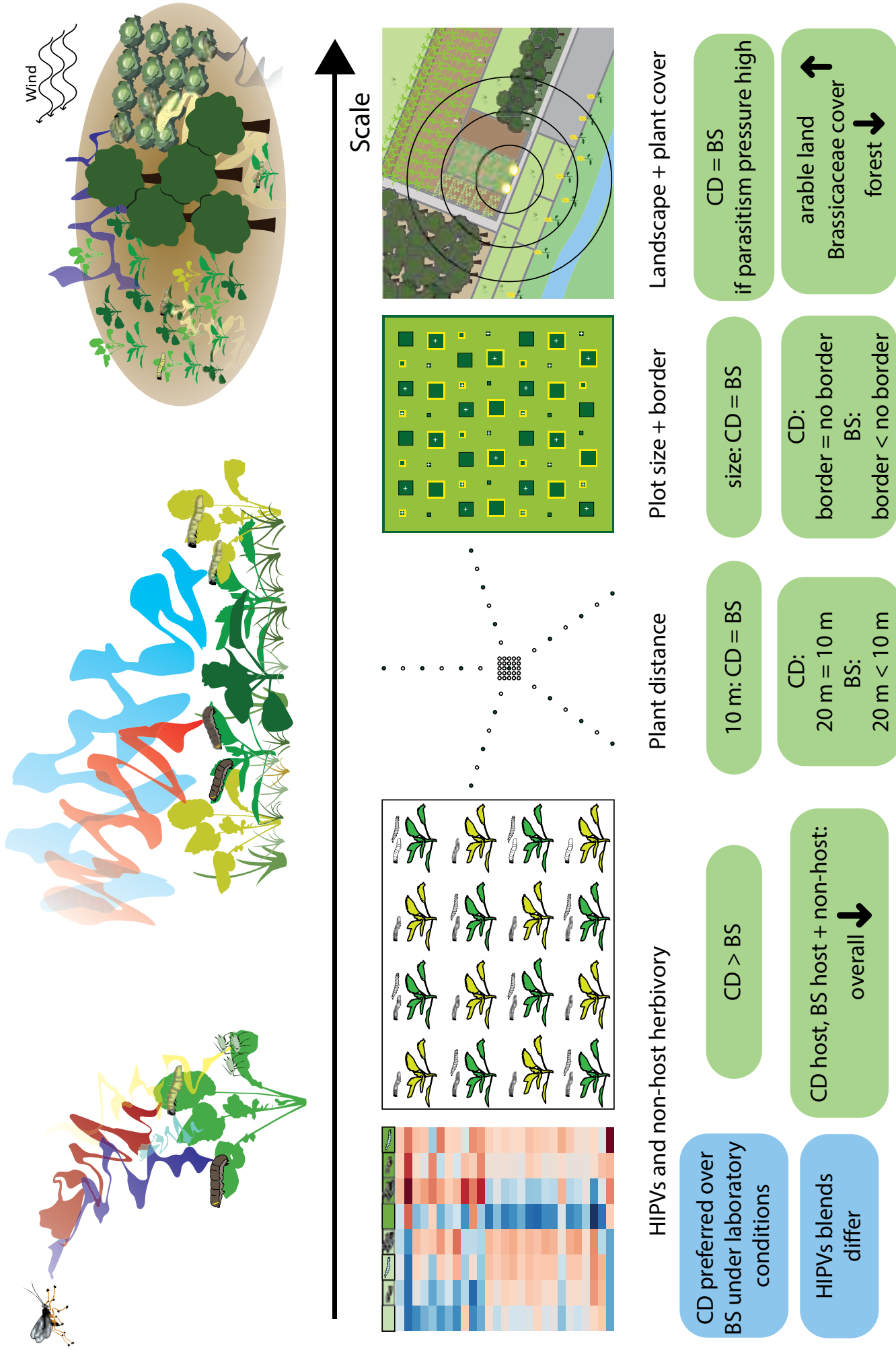

हो हो हो हो
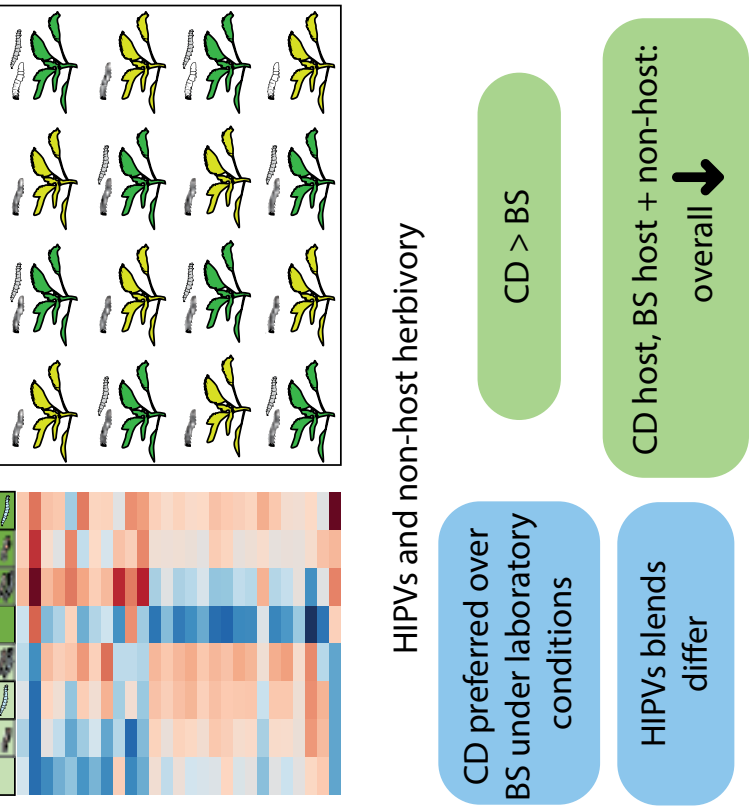
Parasitoids can use herbivore-induced plant volatiles (HIPVs) as long-distance cues to find their herbivorous hosts (Dicke \& Baldwin, 2010; Hare, 2011). While direct cues produced by the host, such as host body odours or frass, provide reliable information on the presence of the host, plant volatiles are produced in larger amounts and may therefore be more detectable from a larger distance (Vet \& Dicke, 1992). The availability and characteristics of these cues can depend on the species or variety of the plant (Gols et al., 2011; Degen et al., 2012), as well as on attributes of the environment in which the parasitoid is foraging (Beyaert \& Hilker, 2014; Wäschke et al., 2014). These can include weather conditions, but also structural and chemical attributes of the vegetation (Blande et al., 2014; Wäschke et al., 2014). Chemical cues mediating interactions between plants, herbivores and parasitoids, as well as other community members, may structure food webs as a result of information exchange between different trophic levels (Vos et al., 2006).

In light of this thesis, I explored HIPV-mediated interactions between plants, herbivores and parasitoids on different spatial scales. I used a suite of methods to assess the role of HIPVs in host finding by parasitoids across spatial scales. Having analysed such factors, I maintain that the spatial context HIPVproducing plants are embedded in can affect the outcome of HIPV-mediated tritrophic interactions in terms of parasitism rates. Figure 1 summarizes the main elements of this thesis, ordered by increasing spatial scale. In this chapter, I will discuss my findings and connect the results of the individual chapters in an attempt to come to some definite answers as to the implications thereof. The first subsection demonstrates how the different spatial scales connect in

Figure 1: Summary of the results of this thesis. From left to right, the experiments of this thesis are arranged from local scale (left) to landscape scale (right). The lowest scale is covered in Chapter 5. This chapter presents herbivore-induced plant volatile (HIPV) profiles of the accessions Christmas Drumhead (CD) and Badger Shipper (BS) and analyses the influence of host and non-host herbivory on foraging efficiency of Cotesia glomerata. Chapter 3 (plant distance) presents the effects of increased interplant distance on parasitism rates. The effects of plot size and presence of a border are studied in Chapter 4. Finally the largest scale is covered in Chapter 6, which presents effects of the surrounding landscape. Green boxes show the main findings of the experiments that are illustrated above the box. A dashed line around the box represents the outcome of comparing the two accessions, while non-bordered boxes represent effects of environmental characteristics on parasitism rates within accession. 
terms of the 'volatile mosaic'-concept. This is followed by a discussion of the role of plant-trait variation in attraction of parasitoids, in which I elaborate on how HIPVs can be a plant resistance or defence trait in complex environments. The fact that the use of plant varieties with elevated levels of HIPV emission is often discussed in research literature in the field (Dicke et al., 1990; Kappers et al., 2011; Heil, 2014) informed the decision to also include a discussion of the results in the context of possible applications in agriculture. The last subsection issues my final conclusions and sheds some light on future perspectives.

\section{The volatile mosaic at different spatial scales and vegetation backgrounds}

In Chapter 2, I argued that 'volatile mosaics' influence parasitoid choices and movement in the field. In this section, I discuss my findings in the context of the volatile mosaic and, by extension, demonstrate how the volatile mosaic can be a helpful ecological concept to explain tritrophic interactions.

In Chapter 3, I investigated whether the distance between host-infested plants influenced parasitism rates under field and semi-field conditions. The distance between HIPV-emitting plants was identified to be important for parasitoids to find the plant. With increasing distance, the proportion of parasitoids that successfully found an infested plant declined. After leaving the plant, HIPV plumes move away from the source in a process influenced by turbulence and other meteorological conditions, leading to the dilution and degradation of compounds in the plume with increasing distance (Murlis et al., 1992; Beyaert \& Hilker, 2014; Blande et al., 2014; Aartsma et al., 2017). Therefore, I can be concluded that distance is an important determinant of the strength and quality of HIPV cues. This also goes to show that a plant that is preferred under laboratory conditions attracts parasitoids from further away. Thus, attractiveness of HIPVs helps parasitoids perceive and navigate towards the plant even when the distance is substantial. The more attractive accessions may produce volatile blends, which tend to break down less easily and which may get transported to more remote locations due to turbulence (Murlis et al., 1992; Blande et al., 2014). 
Neighbouring plants can also contribute to the volatile mosaic by emitting HIPVs or background odours (Chapter 4). Having a border surrounding a patch of plants, some of which are infested with herbivores, and thus emit HIPVs, may interfere with host finding by structural effects or through the chemical mixing of HIPVs of the focal plant and other HIPV plumes. Complex vegetation is an important contributor to the volatile mosaic, because it emits background odours (Randlkofer et al., 2010b; Wäschke et al., 2014). While not much is known about mixing of odour plumes and how such mixing affect insect behaviour (Dicke et al., 2003), research indicates that background odours can result in decreased response towards volatiles (Schröder \& Hilker, 2008; Beyaert \& Hilker, 2014; Deisig et al., 2014). Additionally, variation in species composition of the insect community can alter HIPVs from the source and interact with HIPVs emitted from neighbouring plants with different herbivores (Chapter 5, Bukovinszky et al., 2012; de Rijk et al., 2013). Different herbivore species mixed with the host of the parasitoid can result in alteration of HIPV blends and might mask the presence of the host (Vos et al., 2006) or, alternatively, increase the contrast between host-infested plants and plants which are not of interest (Soler et al., 2007). Recently, it was shown that presence of another herbivore species ( $P$. rapae) reduced parasitism of the aphid Brevicoryne brassicae on broccoli at the field scale, resulting in higher aphid densities in fields where both herbivores were present (Blubaugh et al., 2018). My thesis furthers these claims by demonstrating for the first time that the combination of host and non-host herbivores has different consequences for parasitoid foraging efficiency depending on the plant accession on which these herbivores are present.

In Chapter 2, the landscape scale was identified as the scale which is currently the most lacking in knowledge with regards to the volatile mosaic. Parasitism rates of $P$. brassicae on introduced cabbage patches were affected by features of the landscape and HIPV-mediated parasitoid attraction (Chapter 6). Coverage of alternative host plants is an important determinant of parasitism rates, which is probably related to populations of the host and parasitoid. Alternatively, the abundance of - both wild and cultivated - alternative host plants may function as a corridor within or between crop fields, facilitating dispersal of a parasitoid (Cronin, 2003a; Murakami et al., 2008). Herbivore-induced plant volatiles also have the potential to help establish connectivity between plant patches. More 
research is needed on the spatial range of attraction of HIPVs of wild plants to assess the proper scale of patch spacing for corridors.

\section{Plant-trait variation in attraction of parasitoids}

In all experiments of this thesis, the white cabbage accession Christmas Drumhead was either more attractive, or equally attractive to $C$. glomerata as the accession Badger Shipper, with the exception of the choice between a Badger Shipper plant with hosts and a Christmas Drumhead plant with only the non-host Plutella xylostella (Chapter 5). This indicates that the effect of plant-trait variation in attraction of parasitoids or parasitism rates of herbivores by this parasitoid is consistent over studies. There have been some studies in which the emission of volatiles from plants was modified, where this modification did not lead to strong positive effects on parasitism rates in the field (von Mérey et al., 2012; Bruce et al., 2015). I have also shown that increased attractiveness to parasitoids may result in higher parasitism rates depending on the context in which the plant is present. This mostly pertains to situations which can be interpreted as more complex, e.g. wider plant spacing, greater vegetation and insect community diversity. An exception to this is the landscape scale, where nearby monoculture crop fields of organic farms supported the highest parasitism rates and also had the largest difference in parasitism rates between the accessions (Chapter 6). In this landscape-scale study the underlying mechanism may be related to the number of parasitoids foraging for hosts, while in the other studies parasitoid populations were similar across accession treatments. (Chapters 3, 4, and 5).

The finding that the effect of habitat context on parasitoid foraging behaviour and parasitism rates often depended on which accession was used. This is important when designing experiments on foraging behaviour of insects using cues from the plant. For example, parasitoids released in wagon wheel set-ups with the accession Christmas Drumhead parasitized a proportion of hosts independent of whether host-infested-plant spacing was $10 \mathrm{~m}$ or $20 \mathrm{~m}$, while in set-ups with Badger Shipper parasitism rates were much lower at $20 \mathrm{~m}$ than at $10 \mathrm{~m}$ (Chapter 3). Similarly, the addition of a border surrounding the plant 
had no effect on parasitism rates on Christmas Drumhead, while parasitism rates on Badger Shipper were lower than in plots without border (Chapter 4). In the landscape study, only minor positive effects of Brassicaceae cover or arable land were found for the accession Badger Shipper, while larger effects were found on the accession Christmas Drumhead (Chapter 6). If plant-trait variation in attractiveness to parasitoids had not been taken into account, and if only one accession had been used instead of two, the effects of habitat context would have been different - depending on the chosen accession - and would have inspired different conclusions. This thesis shows that habitat context has different consequences for parasitoid foraging efficiency depending on the studied plant accession.

\section{The spatial context of HIPVs as a defence/resistance trait}

Plants can defend themselves against herbivore attackers with a wide range of defensive traits, including attracting the third trophic level (Price et al., 1980; Schoonhoven et al., 2005). If HIPVs function as a defence trait, there should be a fitness benefit for the plant (Kessler \& Heil, 2011; Poelman, 2015). However, other community members can respond to HIPVs as well (Stam et al., 2014); HIPVs can be used by other herbivores to find suitable food plants (Poelman et al., 2010), repel parasitoids (Snoeren et al., 2010) and attract hyperparasitoids (Zhu et al., 2015). The attraction of other insects which are not beneficial for the plant might make HIPVs ecologically costly as a defence and attenuate potential fitness benefits of HIPVs (Kessler \& Heil, 2011). Alternatively, other herbivores can be repelled by HIPVs, which might amplify potential fitness benefits of HIPVs (De Moraes et al., 2001; Bernasconi et al., 2003). Therefore, it is difficult to examine the contribution of a trait affecting a single interaction to plant fitness in a community context (Strauss et al., 2004; Kessler \& Heil, 2011; Poelman, 2015).

In this thesis, I show that parasitism rates as an outcome of HIPV-mediated tritrophic interactions were influenced by the spatial context around HIPVproducing plants are present and depend on the relative attractiveness of nearby 
plants. In a number of cases, most notably in the more simple situations, with short interplant distance and low vegetation diversity, plant-trait variation of parasitoid attraction did not significantly increase parasitism rates of herbivores on the plant. In these cases, increased attraction of parasitoids via HIPVs cannot be expected to result in a fitness benefit for the plant compared to plants without increased parasitoid attraction. Furthermore, whether HIPVs constitute a defence trait and provide a fitness benefit to the plant might also depend on local parasitoid population dynamics. If few parasitoids are searching for hosts, higher attractiveness via HIPVs might not result in higher parasitism rates and therefore provide no advantage over other plants (Chapter 6). In terms of the 'cry for help' concept (Dicke \& Baldwin, 2010; Heil, 2014), crying for help louder does not matter if there is no one to hear the cries. Alternatively, if parasitoid populations are very high, enhanced attractiveness via HIPVs also does not matter (Chapter 4), because all herbivore-infested plants will be visited anyway, if not via HIPVs then via random encounters. However, it remains a question how common such high parasitoid populations are in nature. It is known that parasitoid populations can be very high in crop monocultures to the point where local extinction of the pest occurs (Gols et al., 2005). In species-rich natural ecosystems, parasitoids may not reach such high populations levels as hosts and their food plants are present in less dense stands. In Chapter 6, hostplant cover was much lower in non-crop habitats than in crop-fields, something which could be related to parasitoid population sizes in these areas, although this remains to be investigated.

In this thesis, I assessed parasitoid behaviour and the resulting parasitism rates of caterpillars in terms of egg presence and/or counts. While it is likely that parasitism of caterpillars results in death of the caterpillars, this is not always the case. For example, eggs can be encapsulated, resulting in a healthy caterpillar (Brodeur \& Vet, 1995; Bukovinszky et al., 2009). Additionally, parasitized hosts often continue feeding from the plant and might even feed more, which possibly does not result in a fitness benefit for the plant (Smallegange et al., 2008). Another aspect, which was not studied in this thesis, is the time component of these interactions. The plant and insect community is variable in time and also populations of parasitoids are subject to strong dynamics (Plećaš et al., 2014; 
Stam et al., 2014). Possible defensive traits such as parasitoid attraction through HIPV might be more important in some years or periods during the year than in others depending on the dynamics of the community.

\section{Using plants with elevated HIPV emission in agriculture}

In many reviews, the prospects of plants with elevated HIPV emission - either by pre-existing varieties, selective breeding or genetic manipulation - have been discussed (Dicke et al., 1990; Bottrell et al., 1998; Kaplan, 2012b; Penaflor \& Bento, 2013; Heil, 2014; Gish et al., 2015; Stenberg et al., 2015; Turlings \& Erb, 2018). Domesticated plants may have lost some of their capacity to respond to herbivory (Chen et al., 2015), although this does not necessarily results in lower HIPV emission (Rowen \& Kaplan, 2016). However, for these varieties to be successful in open-field agricultural settings, we need to know the spatial scale on which HIPVs affect natural enemies (Heil, 2014; Aartsma et al., 2017). After all, if the spatial scale of HIPVs is only small, slightly enhancing this range might not lead to attraction of natural enemies from habitats surrounding a crop field. Existing estimates in the literature on the spatial scale of HIPVs and effects on natural enemy abundance do not exceed a distance of $8 \mathrm{~m}$ (Mallinger et al., 2011; Braasch \& Kaplan, 2012). However, for the more attractive cabbage variety used in this thesis, this distance is at least $20 \mathrm{~m}$ (Chapter 3). This can be considered a promising distance for application in agricultural fields because it attracts parasitoids from a wide 'catchment area'. Through plant selective breeding, perhaps even larger distances might be attained, which might make these varieties even better in an agricultural context. However, the perceptual range and dispersal capacity of the parasitoid have to be taken into account when breeding for increased spatial range of HIPV information.

The augmentation of biological control in open field agriculture through landscape management and creating 'pest-suppressive' landscapes requires sufficiently sized populations of natural enemies to attract them to crop fields when they are needed. However, insect populations in agriculture-dominated areas in Europe are declining, which may have widespread consequences for ecosystem services 
in these areas (Potts et al., 2010; Hallmann et al., 2017; Kleijn et al., 2018). Higher trophic levels often disappear faster from food webs in the case of habitat loss and fragmentation than lower trophic levels (Fahrig, 2003; van Nouhuys, 2005; Dobson et al., 2006). Fragmentation and removal small-scale connecting corridors such as hedgerows or unmanaged field edges lead to greater isolation of habitat patches. Animals in these patches may risk local extinction (Tscharntke et al., 2005a; Haddad et al., 2015; Holland et al., 2016). While crop fields can provide resources for natural enemies, and the building up of populations, they are often not permanent and resource continuity is low (Schellhorn et al., 2015). Conservation biological control measures aimed to increase natural enemy populations are often at the field scale. These measures constitute small-scale improvements such as flower strips, unmown grass strips and local reduction of pesticide use (Jonsson et al., 2015). The 'intermediate landscape complexity hypothesis' predicts that such measurements are most effective in landscapes where some complexity is still present (Tscharntke et al., 2012). They are not effective, however, in the most simple landscapes where source populations of natural enemies are too low (Tscharntke et al., 2012; Jonsson et al., 2015). In the most simple landscapes which have almost no semi-natural habitat left, local-scale measures aimed at conserving natural enemies may not be effective and discourage farmers to give up farm land for habitat creation for farmland biodiversity (Tscharntke et al. 2012). Therefore, large-scale regional programmes will be needed to prioritise areas where management is needed to support and establish communities of natural enemies and other beneficial insects, such as pollinators. Establishing corridors between habitats can be part of these programmes, for which HIPV emitting plants may be instrumental.

Ultimately, the use of plant varieties which are more attractive to natural enemies via HIPVs should lead to higher crop yield if they are to be used in agriculture. This might not necessarily be the case due to the complex nature of insect-plant interactions that include many members of the insect community, including different herbivore species which may also be attracted to HIPVs, and insects of higher trophic levels, such as hyperparasitoids, which may compromise the pestsuppressive effect of parasitoids, as described earlier in this chapter. Usually there are more than one pest species and enemy species that might not all be affected by HIPVs in the same way. Furthermore, management practices of farmers, 
such as pesticide use, may attenuate potential benefits of attracting natural enemies. For organic farmers who depend on natural enemies for pest control, using an attractive plant variety in a landscape with sufficient natural enemy populations might lead to yield improvements. A next step in continuing the research of this thesis would be experiments at the farm level which incorporate management practices and yield measurements. This will provide novel insights in the contribution of increased parasitoid attraction to crop yield.

\section{Conclusions and future research perspectives}

Parasitoids often forage in heterogeneous habitats and make use of chemical cues to find their hosts. Spatial attributes of these habitats can have major impacts on parasitoid foraging efficiency, which results in different parasitism pressures throughout the landscape and allows herbivores to escape parasitism by colonizing less 'apparent' patches. Based on the results of this thesis, I conclude that both local- and landscape-scale spatial processes are important for tritrophic interactions. How these spatial processes influence tritrophic interactions depends partly on the strength and quality of the information provided by the first trophic level, the focal plant, via HIPVs.

In this thesis, I investigated parasitoid movement and host-finding success using HIPVs in an indirect way, by using two different cabbage accessions known to differ in attraction of these parasitoids. While the outcomes of my research show that parasitoid attractiveness via HIPVs is important for attraction of parasitoids at different spatial scales, there are still many open questions regarding how HIPVs move through the environment after leaving the plant. How long do HIPV plumes persist in the atmosphere and in a way insects will be able to recognize it? How do neighbouring plants interfere with HIPVs and how does the identity of the 'sending' plant alter this interference? Quantifying HIPVs in real-time and under field conditions is a methodologically difficult task, however, especially when it comes to sampling air which is not in the direct vicinity of the plant, let alone $20 \mathrm{~m}$ away from the source. Alternatively, it is also possible to investigate the response of the parasitoid rather than HIPVs directly. This can 
be done by using a portable electroantennogram (EAG) device to test antennal response of insects downwind from a plant (Andersson et al., 2013). However, due to the methodological difficulties in measuring volatiles and insect responses in the field, the indirect way of comparing two plant accessions is a practical way of studying how HIPVs influence parasitoid movement and behaviour in the field.

In this thesis I focussed on plant-trait variation in HIPVs and effects of habitat complexity at different spatial scales on host-finding efficiency of mostly a single parasitoid species, C. glomerata. However, besides plant traits, traits of the parasitoid also determine how the volatile mosaic is perceived by this parasitoid. For example, parasitoids might differ in their ability to detect volatile compounds at certain concentrations (Gouinguené et al., 2005). Parasitoids being more sensitive to low concentrations of volatiles could result them in being able to perceive plants from a greater distance. However, in order to be able to do so, parasitoids will need to have the dispersal capacity to follow odour plumes to their source (van Nouhuys \& Ehrnsten, 2004). Little is known on variation between parasitoid species in sensitivity to different concentration of HIPVs, their threshold concentrations and the maximum distance from the source where this threshold concentration can be found in the field. Differences in this perceptual range can be important for parasitoid foraging behaviour at the landscape scale (Olden et al., 2004; Bianchi et al., 2009; Vinatier et al., 2011b). Additionally, parasitoids are able to learn cues from host-infested plants, which can improve their foraging efficiency (Smid \& Vet, 2016). Oviposition experience on a less preferred plant can result in increased response towards volatiles of that plant (Liu \& Jiang, 2003) and also partially counter negative effects of habitat complexity (Kruidhof et al., 2015). This may also partly explain why differences in plant attractiveness are sometimes found under laboratory conditions which use naive parasitoids, but not in field conditions (Bruce et al., 2015). Future studies could expand upon this thesis by also considering parasitoid traits such as dispersal capacity, perceptual range and learning ability, in order to understand their role in parasitoid foraging behaviour in complex situations. This can be done by investigating different species of parasitoids which are known to differ in one of the traits mentioned above. 
It is important to study parasitoid foraging behaviour in field situations to evaluate the relevance of phenomena found under laboratory conditions (Heimpel \& Casas, 2008). This thesis makes an important contribution to the fundamental knowledge of foraging behaviour under field conditions and, as such, underlines the relevance of HIPVs in mediating tritrophic interactions in natural and agricultural systems. Such knowledge is valuable for developing agricultural practices that do not rely on insecticides but rather on biological control of insect pests.

\section{Acknowledgements}

I thank Erik Poelman, Wopke van der Werf, Felix Bianchi and Marcel Dicke for their constructive comments on an earlier version of this chapter. 
.

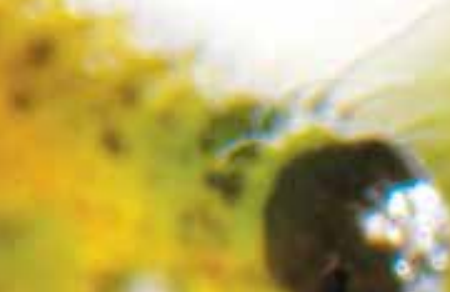

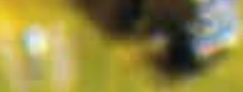
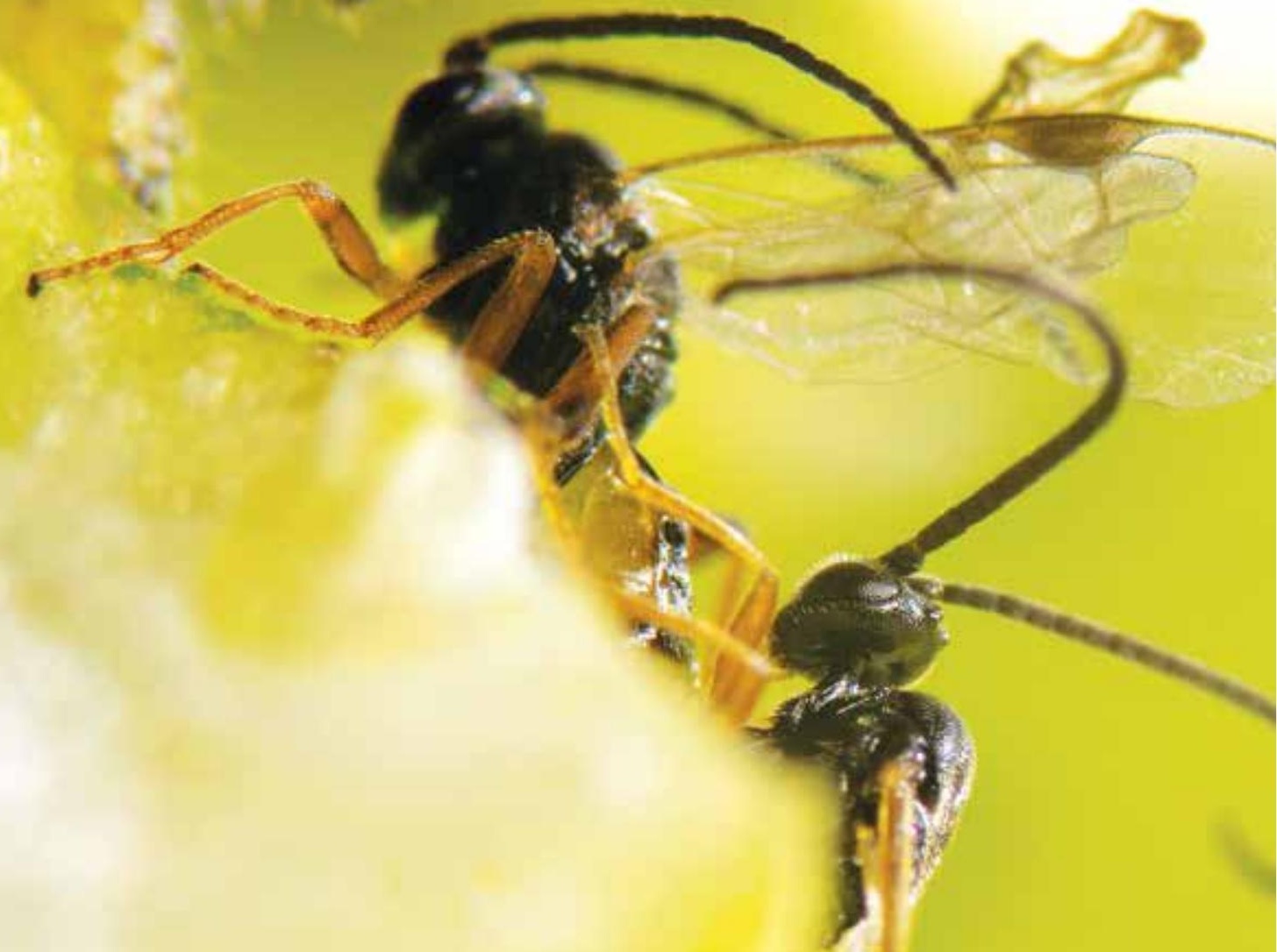
References 
Aartsma Y, Leroy B, van der Werf W, Dicke M, Poelman EH, Bianchi FJJA. 2018. Intraspecific variation in herbivore-induced plant volatiles influences the spatial range of plant-parasitoid interactions. Accepted for publication in Oikos, doi:10.1111/oik.05151

Aartsma Y, Bianchi FJJA, van der Werf W, Poelman EH, Dicke M. 2017. Herbivore-induced plant volatiles and tritrophic interactions across spatial scales. New Phytologist 216(4): 1054-1063.

Abrams PA, Matsuda H. 1996. Positive indirect effects between prey species that share predators. Ecology 77(2): 610-616.

Adams RP. 2001. Identification of essential oil components by gas chromatography/mass spectrometry. Carol Stream: Allured Publishing Corporation.

Áhman I, Glinwood R, Ninkovic V. 2010. The potential for modifying plant volatile composition to enhance resistance to arthropod pests. CAB Reviews: Perspectives in Agriculture, Veterinary Science, Nutrition and Natural Resources 5(006): 1-10.

Amo L, Jansen JJ, Dam NM, Dicke M, Visser ME. 2013. Birds exploit herbivore-induced plant volatiles to locate herbivorous prey. Ecology Letters 16(11): 1348-1355.

Andersson P, Lofstedt C, Hamback PA. 2013. How insects sense olfactory patches - the spatial scaling of olfactory information. Oikos 122(7): 1009-1016.

Arim M, Marquet PA. 2004. Intraguild predation: a widespread interaction related to species biology. Ecology Letters 7(7): 557-564.

Arimura G-i, Köpke S, Kunert M, Volpe V, David A, Brand P, Dabrowska P, Maffei ME, Boland W. 2008. Effects of feeding Spodoptera littoralis on lima bean leaves: IV. Diurnal and nocturnal damage differentially initiate plant volatile emission. Plant Physiology 146(3): 965-973.

Barbosa P, Hines J, Kaplan I, Martinson H, Szczepaniec A, Szendrei Z. 2009. Associational resistance and associational susceptibility: having right or wrong neighbors. Annual Review of Ecology, Evolution, and Systematics 40(1): 1-20.

Barton K 2016. Multi-Model Inference. R package version 1.15. 6.

Bates D, Maechler M, Bolker B, Walker S. 2015. Fitting linear mixed-effects models using Ime4. Journal of Statistical Software 67(1): 1-48.

Bau J, Cardé RT. 2015. Modeling optimal strategies for finding a resource-linked, windborne odor plume: Theories, robotics, and biomimetic lessons from flying insects. Integrative and Comparative Biology 55(3): 461-477.

Bernasconi ML, Turlings TCJ, Ambrosetti L, Bassetti P, Dorn S. 2003. Herbivore-induced emissions of maize volatiles repel the corn leaf aphid, Rhopalosiphum maidis. Entomologia Experimentalis et Applicata 87(2): 133-142.

Bernasconi Ockroy ML, Turlings TCJ, Edwards PJ, Fritzsche-Hoballah ME, Ambrosetti L, Bassetti P, Dorn S. 2001. Response of natural populations of predators and parasitoids to artificially induced volatile emissions in maize plants (Zea mays L.). Agricultural and Forest Entomology 3(3): 201-209.

Beyaert I, Hilker M. 2014. Plant odour plumes as mediators of plant-insect interactions. Biological Reviews 89(1): 68-81.

Bezemer TM, Harvey JA, Kamp AFD, Wagenaar R, Gols R, Kostenko O, Fortuna T, Engelkes T, Vet LEM, van der Putten WH, et al. 2010. Behaviour of male and female parasitoids in the field: influence of patch size, host density, and habitat complexity. Ecological Entomology 35(3): 341-351.

Bianchi FJJA, Schellhorn NA, Cunningham SA. 2012. Habitat functionality for the ecosystem service of pest control: reproduction and feeding sites of pests and natural enemies. Agricultural and Forest Entomology 15(1): 12-23.

Bianchi FJJA, Schellhorn NA, van der WerfW. 2009. Foraging behaviour of predators in heterogeneous landscapes: the role of perceptual ability and diet breadth. Oikos 118(9): 1363-1372.

Bianchi FJJA, Wäckers FL. 2008. Effects of flower attractiveness and nectar availability in field margins on biological control by parasitoids. Biological Control 46(3): 400-408.

Bianchi FJJA, Goedhart PW, Baveco JM. 2008. Enhanced pest control in cabbage crops near forest in the Netherlands. Landscape Ecology 23(5): 595-602. 
Bianchi F, Booij C, Tscharntke T. 2006. Sustainable pest regulation in agricultural landscapes: a review on landscape composition, biodiversity and natural pest control. Proceedings of the Royal Society of London B: Biological Sciences 273(1595): 1715-1727.

Blande JD, Holopainen JK, Niinemets Ü. 2014. Plant volatiles in polluted atmospheres: stress responses and signal degradation. Plant, Cell \& Environment 37(8): 1892-1904.

Blubaugh CK, Asplund JS, Eigenbrode SD, Morra MJ, Philips CR, Popova IE, Reganold JP, Snyder WE. 2018. Dual-guild herbivory disrupts predator-prey interactions in the field. Ecology 99(5): 1089-1098.

Bottrell DG, Barbosa P, Gould F. 1998. Manipulating natural enemies by plant variety selection and modification: a realistic strategy? Annual Review of Entomology 43(1): 347-367.

Bowler DE, Benton TG. 2005. Causes and consequences of animal dispersal strategies: relating individual behaviour to spatial dynamics. Biological Reviews 80(2): 205-225.

Braasch J, Kaplan I. 2012. Over what distance are plant volatiles bioactive? Estimating the spatial dimensions of attraction in an arthropod assemblage. Entomologia Experimentalis et Applicata 145(2): 115-123.

Brodeur J, Geervliet Jacqueline BF, Vet Louise EM. 1996. The role of host species, age and defensive behaviour on ovipositional decisions in a solitary specialist and gregarious generalist parasitoid (Cotesia species). Entomologia Experimentalis Et Applicata 81(2): 125-132.

Brodeur J, Vet LEM. 1995. Relationships between parasitoid host range and host defence: a comparative study of egg encapsulation in two related parasitoid species. Physiological Entomology 20(1): 7-12.

Bruce TJA, Aradottir GI, Smart LE, Martin JL, Caulfield JC, Doherty A, Sparks CA, Woodcock CM, Birkett MA, Napier JA, et al. 2015. The first crop plant genetically engineered to release an insect pheromone for defence. Scientific Reports 5: 11183.

Bruinsma M, Lucas-Barbosa D, ten Broeke CJM, van Dam NM, van Beek TA, Dicke M, van Loon JJA. 2014. Folivory affects composition of nectar, floral odor and modifies pollinator behavior. Journal of Chemical Ecology 40(1): 39-49.

Bukovinszky T, Poelman EH, Kamp A, Hemerik L, Prekatsakis G, Dicke M. 2012. Plants under multiple herbivory: consequences for parasitoid search behaviour and foraging efficiency. Animal Behaviour 83(2): 501-509.

Bukovinszky T, Poelman EH, Gols R, Prekatsakis G, Vet LEM, Harvey JA, Dicke M. 2009. Consequences of constitutive and induced variation in plant nutritional quality for immune defence of a herbivore against parasitism. Oecologia 160(2): 299-308.

Bukovinszky T, van Veen FJF, Jongema Y, Dicke M. 2008. Direct and indirect effects of resource quality on food web structure. Science 319(5864): 804-807.

Bukovinszky T, Gols R, Hemerik LIA, van Lenteren JC, Vet LEM. 2007. Time allocation of a parasitoid foraging in heterogeneous vegetation: implications for host-parasitoid interactions. Journal of Animal Ecology 76(5): 845-853.

Bukovinszky T, Potting RPJ, Clough Y, van Lenteren JC, Vet LEM. 2005. The role of pre- and postalighting detection mechanisms in the responses to patch size by specialist herbivores. Oikos 109(3): 435-446.

Cardé RT, Willis MA. 2008. Navigational strategies used by insects to find distant, wind-borne sources of odor. Journal of Chemical Ecology 34(7): 854-866.

Chabaane Y, Laplanche D, Turlings TCJ, Desurmont GA. 2015. Impact of exotic insect herbivores on native tritrophic interactions: a case study of the African cotton leafworm, Spodoptera littoralis and insects associated with the field mustard Brassica rapa. Journal of Ecology 103(1): 109-117.

Chaplin-Kramer R, de Valpine P, Mills NJ, Kremen C. 2013. Detecting pest control services across spatial and temporal scales. Agriculture Ecosystems \& Environment 181: 206-212.

Chaplin-Kramer R, O'Rourke ME, Blitzer EJ, Kremen C. 2011. A meta-analysis of crop pest and natural enemy response to landscape complexity. Ecology Letters 14(9): 922-932.

Chen YH, Gols R, Stratton CA, Brevik KA, Benrey B. 2015. Complex tritrophic interactions in response to crop domestication: predictions from the wild. Entomologia Experimentalis et Applicata 157(1): 40-59. 
Clavijo McCormick A, Unsicker SB, Gershenzon J. 2012. The specificity of herbivore-induced plant volatiles in attracting herbivore enemies. Trends in Plant Science 17(5): 303-310.

Clobert J, Galliard L, Cote J, Meylan S, Massot M. 2009. Informed dispersal, heterogeneity in animal dispersal syndromes and the dynamics of spatially structured populations. Ecology Letters 12(3): 197-209.

Cortesero AM, Stapel JO, Lewis WJ. 2000. Understanding and manipulating plant attributes to enhance biological control. Biological Control 17(1): 35-49.

Costamagna AC, Menalled FD, Landis DA. 2004. Host density influences parasitism of the armyworm Pseudaletia unipuncta in agricultural landscapes. Basic and Applied Ecology 5(4): 347-355.

Crist TO, Pradhan-Devare SV, Summerville KS. 2006. Spatial variation in insect community and species responses to habitat loss and plant community composition. Oecologia 147(3): 510521.

Cronin JT, Reeve JD. 2005. Host-parasitoid spatial ecology: a plea for a landscape-level synthesis. Proceedings of the Royal Society B - Biological Sciences 272(1578): 2225-2235.

Cronin JT. 2003a. Matrix heterogeneity and host-parasitoid interactions in space. Ecology 84(6): 1506-1516.

Cronin JT. 2003b. Patch structure, oviposition behavior, and the distribution of parasitism risk. Ecological Monographs 73(2): 283-300.

D'Alessandro M, Turlings TC. 2006. Advances and challenges in the identification of volatiles that mediate interactions among plants and arthropods. Analyst 131(1): 24-32.

De Moraes CM, Mescher MC, Tumlinson JH. 2001. Caterpillar-induced nocturnal plant volatiles repel conspecific females. Nature 410: 577.

de Rijk M, Cegarra Sánchez V, Smid HM, Engel B, Vet LEM, Poelman EH. 2018. Associative learning of host presence in non-host environments influences parasitoid foraging. Ecological Entomology 43(3): 318-325.

de Rijk M, Wang Q, Papagiannaki E, Dicke M, Poelman EH. 2016a. Herbivore species identity rather than diversity of the non-host community determines foraging behaviour of the parasitoid wasp Cotesia glomerata. Entomologia Experimentalis et Applicata 161(1): 20-30.

de Rijk M, Yang D, Engel B, Dicke M, Poelman Erik H. 2016b. Feeding guild of non-host community members affects host-foraging efficiency of a parasitic wasp. Ecology 97(6): 1388-1399.

de Rijk M, Dicke M, Poelman EH. 2013. Foraging behaviour by parasitoids in multiherbivore communities. Animal Behaviour 85(6): 1517-1528.

Degen T, Bakalovic N, Bergvinson D, Turlings TCJ. 2012. Differential performance and parasitism of caterpillars on maize inbred lines with distinctly different herbivore-induced volatile emissions. PLOS ONE 7(10): e47589.

Degen T, Dillmann C, Marion-Poll F, Turlings TCJ. 2004. High genetic variability of herbivore-induced volatile emission within a broad range of maize inbred lines. Plant Physiology 135(4): 19281938.

Deisig N, Dupuy F, Anton S, Renou M. 2014. Responses to pheromones in a complex odor world: sensory processing and behavior. Insects 5(2): 399-422.

Desurmont GA, Guiguet A, Turlings TCJ. 2018. Invasive insect herbivores as disrupters of chemicallymediated tritrophic interactions: effects of herbivore density and parasitoid learning. Biological Invasions 20(1): 195-206.

Desurmont GA, Harvey J, van Dam NM, Cristescu SM, Schiestl FP, Cozzolino S, Anderson P, Larsson MC, Kindlmann P, Danner H, et al. 2014. Alien interference: disruption of infochemical networks by invasive insect herbivores. Plant, Cell \& Environment 37(8): 1854-1865.

Dicke M, Baldwin IT. 2010. The evolutionary context for herbivore-induced plant volatiles: beyond the 'cry for help'. Trends in Plant Science 15(3): 167-175.

Dicke M, van Loon JJ, Soler R. 2009. Chemical complexity of volatiles from plants induced by multiple attack. Nature Chemical Biology 5(5): 317-324.

Dicke M, de Boer JG, Höfte M, Rocha-Granados MC. 2003. Mixed blends of herbivore-induced plant volatiles and foraging success of carnivorous arthropods. Oikos 101(1): 38-48. 
Dicke M, Sabelis MW, Takabayashi J, Bruin J, Posthumus MA. 1990. Plant strategies of manipulating predator-prey interactions through allelochemicals: prospects for application in pest control. Journal of Chemical Ecology 16: 3091-3118.

Dinno A. 2017. dunn.test: Dunn's test of multiple comparisons using rank sums.

Dobson A, Lodge D, Alder J, Cumming Graeme S, Keymer J, McGlade J, Mooney H, Rusak James A, Sala O, Wolters V, et al. 2006. Habitat loss, trophic collapse, and the decline of ecosystem services. Ecology 87(8): 1915-1924.

Edger PP, Heidel-Fischer HM, Bekaert M, Rota J, Glöckner G, Platts AE, Heckel DG, Der JP, Wafula EK, Tang M. 2015. The butterfly plant arms-race escalated by gene and genome duplications. Proceedings of the National Academy of Sciences 112(27): 8362-8366.

Elzinga JA, van Nouhuys S, van Leeuwen D-J, Biere A. 2007. Distribution and colonisation ability of three parasitoids and their herbivorous host in a fragmented landscape. Basic and Applied Ecology 8(1): 75-88.

Engelberth J, Alborn HT, Schmelz EA, Tumlinson JH. 2004. Airborne signals prime plants against insect herbivore attack. Proceedings of the National Academy of Sciences of the United States of America 101(6): 1781-1785.

Erb M, Foresti N, Turlings TC. 2010. A tritrophic signal that attracts parasitoids to host-damaged plants withstands disruption by non-host herbivores. BMC Plant Biology 10:247.

Fahrig L, Baudry J, Brotons L, Burel Françoise G, Crist Thomas O, Fuller Robert J, Sirami C, Siriwardena Gavin M, Martin JL. 2010. Functional landscape heterogeneity and animal biodiversity in agricultural landscapes. Ecology Letters 14(2): 101-112.

Fahrig L. 2003. Effects of habitat fragmentation on biodiversity. Annual Review of Ecology, Evolution, and Systematics 34(1): 487-515.

Fan M, Zhang B, Li MY. 2010. Mechanisms for stable coexistence in an insect community. Mathematical Biosciences and Engineering 7: 603-622.

Feng Y, Kravchuk O, Sandhu H, Wratten SD, Keller MA. 2017. The activities of generalist parasitoids can be segregated between crop and adjacent non-crop habitats. Journal of Pest Science 90(1): 275-286.

Finch S, Billiald H, Collier RH. 2003. Companion planting - do aromatic plants disrupt host-plant finding by the cabbage root fly and the onion fly more effectively than non-aromatic plants? Entomologia Experimentalis et Applicata 109(3): 183-195.

Finnerty PB, Stutz RS, Price CJ, Banks PB, McArthur C. 2017. Leaf odour cues enable non-random foraging by mammalian herbivores. J Anim Ecol 86(6): 1317-1328.

Fischer S, Samietz J, Wäckers F, Dorn S. 2001. Interaction of vibrational and visual cues in parasitoid host location. Journal of Comparative Physiology A 187(10): 785-791.

Forbes AA, Bagley RK, Beer MA, Hippee AC, Widmayer HA. 2018. Quantifying the unquantifiable: why Hymenoptera - not Coleoptera - is the most speciose animal order. bioRxiv https://doi. org/10.1101/274431

Fox J, Weisberg S. 2011. An $\{R\}$ Companion to applied Regression, second Edition. Thousand Oaks CA: Sage.

Friberg M, Posledovich D, Wiklund C. 2015. Decoupling of female host plant preference and offspring performance in relative specialist and generalist butterflies. Oecologia 178(4): 1181-1192.

Fryxell JM, Hazell M, Börger L, Dalziel BD, Haydon DT, Morales JM, Mclntosh T, Rosatte RC. 2008. Multiple movement modes by large herbivores at multiple spatiotemporal scales. Proceedings of the National Academy of Sciences 105(49): 19114-19119.

Geervliet JBF, Vreugdenhil Al, Dicke M, Vet LEM. 1998. Learning to discriminate between infochemicals from different plant-host complexes by the parasitoids Cotesia glomerata and C. rubecula. Entomologia Experimentalis et Applicata 86(3): 241-252.

Geervliet JBF, Vet LEM, Dicke M. 1996. Innate responses of the parasitoids Cotesia glomerata and C. rubecula (Hymenoptera: Braconidae) to volatiles from different plant-herbivore complexes. Journal of Insect Behavior 9(4): 525-538.

Geervliet JBF, Vet LEM, Dicke M. 1994. Volatiles from damaged plants as major cues in long-range host-searching by the specialist parasitoid Cotesia rubecula. Entomologia Experimentalis et 
Applicata 73(3): 289-297.

Girling RD, Stewart-Jones A, Dherbecourt J, Staley JT, Wright DJ, Poppy GM. 2011. Parasitoids select plants more heavily infested with their caterpillar hosts: a new approach to aid interpretation of plant headspace volatiles. Proceedings of the Royal Society B: Biological Sciences 278(1718): 2646-2653.

Gish M, De Moraes CM, Mescher MC. 2015. Herbivore-induced plant volatiles in natural and agricultural ecosystems: open questions and future prospects. Current Opinion in Insect Science 9: 1-6.

Godfray HCJ. 1994. Parasitoids: behavioral and evolutionary ecology. Princeton, N.J.: Princeton University Press.

Gohole LS, Overholt WA, Khan ZR, Vet LEM. 2005. Close-range host searching behavior of the stemborer parasitoids Cotesia sesamiae and Dentichasmias busseolae: Influence of a non-host plant Melinis minutiflora. Journal of Insect Behavior 18(2): 149-169.

Gols R, Veenemans C, Potting RPJ, Smid HM, Dicke M, Harvey JA, Bukovinszky T. 2012. Variation in the specificity of plant volatiles and their use by a specialist and a generalist parasitoid. Animal Behaviour 83(5): 1231-1242.

Gols R, Bullock JM, Dicke M, Bukovinszky T, Harvey JA. 2011. Smelling the wood from the trees: Non-linear parasitoid responses to volatile attractants produced by wild and cultivated cabbage. Journal of Chemical Ecology 37(8): 795-807.

Gols R, Bukovinszky T, Hemerik L, Harvey JA, van Lenteren JC, Vet LEM. 2005. Reduced foraging efficiency of a parasitoid under habitat complexity: implications for population stability and species coexistence. Journal of Animal Ecology 74(6): 1059-1068.

González E, Salvo A, Valladares G. 2017. Arthropod communities and biological control in soybean fields: Forest cover at landscape scale is more influential than forest proximity. Agriculture, Ecosystems \& Environment 239: 359-367.

Gouinguené S, Pickett JA, Wadhams LJ, Birkett MA, Turlings TCJ. 2005. Antennal electrophysiological responses of three parasitic wasps to caterpillar-induced volatiles from maize (Zea mays mays), Cotton (Gossypium herbaceum), and Cowpea (Vigna unguiculata). Journal of Chemical Ecology 31(5): 1023-1038.

Gurr GM, Wratten SD, Landis DA, You M. 2017. Habitat management to suppress pest populations: progress and prospects. Annual Review of Entomology 62(1): 91-109.

Haddad NM, Brudvig LA, Clobert J, Davies KF, Gonzalez A, Holt RD, Lovejoy TE, Sexton JO, Austin MP, Collins CD. 2015. Habitat fragmentation and its lasting impact on Earth's ecosystems. Science Advances 1(2): e1500052.

Hagen M, Kissling WD, Rasmussen C, De Aguiar MAM, Brown LE, Carstensen DW, Alves-DosSantos I, Dupont YL, Edwards FK, Genini J, et al. 2012. Biodiversity, species interactions and ecological networks in a fragmented world. Advances in Ecological Research: Academic Press, 89-210.

Halitschke R, Stenberg JA, Kessler D, Kessler A, Baldwin IT. 2008. Shared signals -'alarm calls' from plants increase apparency to herbivores and their enemies in nature. Ecology Letters 11(1): 24-34.

Hallmann CA, Sorg M, Jongejans E, Siepel H, Hofland N, Schwan H, Stenmans W, Müller A, Sumser $\mathrm{H}$, Hörren T, et al. 2017. More than 75 percent decline over 27 years in total flying insect biomass in protected areas. PLOS ONE 12(10): e0185809.

Hambäck PA, Englund G. 2005. Patch area, population density and the scaling of migration rates: the resource concentration hypothesis revisited. Ecology Letters 8(10): 1057-1065.

Hammill E, Kratina P, Vos M, Petchey OL, Anholt BR. 2015. Food web persistence is enhanced by non-trophic interactions. Oecologia 178(2): 549-556.

Hare JD. 2011. Ecological role of volatiles produced by plants in response to damage by herbivorous insects. Annual Review of Entomology 56: 161-180.

Heil M. 2014. Herbivore-induced plant volatiles: targets, perception and unanswered questions. New Phytologist 204(2): 297-306.

Heimpel GE, Casas J. 2008. Parasitoid foraging and oviposition behavior in the field. Behavioral 
ecology of insect parasitoids. Blackwell, Oxford: 51-70.

Heinen R, Gols R, Harvey JA. 2016. Black and garlic mustard plants are highly suitable for the development of two native pierid butterflies. Environmental Entomology 45(3): 671-676.

Hilker M, McNeil J 2008. Chemical and behavioral ecology in insect parasitoids: how to behave optimally in a complex odorous environment. Behavioral Ecology of Insect Parasitoids: Blackwell Publishing Ltd, 92-112.

Hlavac M. 2018. stargazer: Well-Formatted regression and summary statistics tables.

Hoedjes KM, Kruidhof HM, Huigens ME, Dicke M, Vet LEM, Smid HM. 2011. Natural variation in learning rate and memory dynamics in parasitoid wasps: opportunities for converging ecology and neuroscience. Proceedings of the Royal Society B: Biological Sciences.

Holland JM, Bianchi FJ, Entling MH, Moonen AC, Smith BM, Jeanneret P. 2016. Structure, function and management of semi-natural habitats for conservation biological control: a review of European studies. Pest Management Science 72(9): 1638-1651.

Holopainen JK, Gershenzon J. 2010. Multiple stress factors and the emission of plant VOCs. Trends in Plant Science 15(3): 176-184.

Holyoak M, Casagrandi R, Nathan R, Revilla E, Spiegel O. 2008. Trends and missing parts in the study of movement ecology. Proceedings of the National Academy of Sciences 105(49): 19060-19065.

Hothorn T, Bretz F, Westfall P. 2008. Simultaneous inference in general parametric models. Biometric Journal 50(3): 346-363.

Isaacs R, Tuell J, Fiedler A, Gardiner M, Landis D. 2008. Maximizing arthropod-mediated ecosystem services in agricultural landscapes: the role of native plants. Frontiers in Ecology and the Environment 7(4): 196-203.

James DG, Price TS. 2004. Field-testing of methyl salicylate for recruitment and retention of beneficial insects in grapes and hops. Journal of Chemical Ecology 30(8): 1613-1628.

Jonsson M, Straub Cory S, Didham Raphael K, Buckley Hannah L, Case Bradley S, Hale Roddy J, Gratton C, Wratten Steve D. 2015. Experimental evidence that the effectiveness of conservation biological control depends on landscape complexity. Journal of Applied Ecology 52(5): 1274-1282.

Kaiser L, Willis MA, Carde RT. 1994. Flight maneuvers used by a parasitic wasp to locate hostinfested plant. Entomologia Experimentalis et Applicata 70(3): 285-294.

Kamio M, Derby CD. 2017. Finding food: how marine invertebrates use chemical cues to track and select food. Natural Product Reports 34(5): 514-528.

Kaplan I, Lewis D. 2015. What happens when crops are turned on? Simulating constitutive volatiles for tritrophic pest suppression across an agricultural landscape. Pest Management Science 71(1): 139-150.

Kaplan I. 2012a. Attracting carnivorous arthropods with plant volatiles: The future of biocontrol or playing with fire? Biological Control 60(2): 77-89.

Kaplan I. 2012b. Trophic complexity and the adaptive value of damage-induced plant volatiles. PLOS Biology 10(11): e1001437.

Kappers IF, Hoogerbrugge H, Bouwmeester HJ, Dicke M. 2011. Variation in herbivory-induced volatiles among cucumber (Cucumis sativus L.) varieties has consequences for the attraction of carnivorous natural enemies. Journal of Chemical Ecology 37(2): 150-160.

Karban R, Mata Tawny M, Grof-Tisza P, Crutsinger G, Holyoak Marcel A. 2013. Non-trophic effects of litter reduce ant predation and determine caterpillar survival and distribution. Oikos 122(9): 1362-1370.

Karp DS et al. 2018 Crop pests and predators exhibit inconsistent responses to surrounding landscape composition, Proceedings of the National Academy of Sciences DOI: 10.1073/ pnas.1800042115.

Kerguelen V, Cardé RT. 1997. Manoeuvres of female Brachymeria intermedia flying towards hostrelated odours in a wind tunnel. Physiological Entomology 22(4): 344-356.

Kessler A, Heil M. 2011. The multiple faces of indirect defences and their agents of natural selection. Functional Ecology 25(2): 348-357. 
Khan ZR, Ampong-Nyarko K, Chiliswa P, Hassanali A, Kimani S, Lwande W, Overholt WA, Overholt WA, Picketta JA, Smart LE, et al. 1997. Intercropping increases parasitism of pests. Nature 388(6643): 631-632.

Kleijn D, Bink RJ, ter Braak CJ, van Grunsven R, Ozinga WA, Roessink I, Scheper JA, Schmidt AM, de Vries MFW, Wegman R. 2018. Achteruitgang insectenpopulaties in Nederland: trends, oorzaken en kennislacunes: Wageningen Environmental Research.

Kos M, Broekgaarden C, Kabouw P, Oude Lenferink K, Poelman Erik H, Vet Louise EM, Dicke M, van Loon Joop JA. 2011. Relative importance of plant-mediated bottom-up and top-down forces on herbivore abundance on Brassica oleracea. Functional Ecology 25(5): 1113-1124.

Kostenko O, Lammers M, Grootemaat S, Kroon T, Harvey JA, van Geem M, Bezemer TM. 2015. Effects of plant diversity and structural complexity on parasitoid behaviour in a field experiment. Ecological Entomology 40(6): 748-758.

Kremen C. 2005. Managing ecosystem services: what do we need to know about their ecology? Ecology Letters 8(5): 468-479.

Kristensen NP, De Barro PJ, Schellhorn NA. 2013a. The initial dispersal and spread of an intentional invader at three spatial scales. PLOS ONE 8(5): e62407.

Kristensen NP, Schellhorn NA, Hulthen AD, Howie U, Barro PJD. 2013b. Wind-borne dispersal of a parasitoid: The process, the model, and its validation. Environmental Entomology 42(6): 1137-1148.

Kruidhof HM, Roberts AL, Magdaraog P, Munoz D, Gols R, Vet LE, Hoffmeister TS, Harvey JA. 2015. Habitat complexity reduces parasitoid foraging efficiency, but does not prevent orientation towards learned host plant odours. Oecologia 179(2): 353-361.

Kulahci IG, Dornhaus A, Papaj DR. 2008. Multimodal signals enhance decision making in foraging bumblebees. Proceedings of the Royal Society of London B: Biological Sciences 275(1636): 797-802.

Kuroyanagi T, Abe J, Uefune M, Takabayashi J 2012. Computational fluid dynamics simulation of herbivore-induced plant volatiles around greenhouses. Acta Horticulturae: International Society for Horticultural Science (ISHS), Leuven, Belgium. 147-154.

Landis DA, Wratten SD, Gurr GM. 2000. Habitat hanagement to conserve natural enemies of arthropod pests in agriculture. Annual Review of Entomology 45(1): 175-201.

Lewis W, Stapel JO, Cortesero AM, Takasu K. 1998. Understanding how parasitoids balance food and host needs: importance to biological control. Biological Control 11(2): 175-183.

Li T, Blande JD, Holopainen JK. 2016. Atmospheric transformation of plant volatiles disrupts host plant finding. Scientific Reports 6: 33851.

Lima SL, Dill LM. 1990. Behavioral decisions made under the risk of predation: a review and prospectus. Canadian Journal of Zoology 68(4): 619-640.

Liu SS, Jiang LH. 2003. Differential parasitism of Plutella xylostella (Lepidoptera: Plutellidae) larvae by the parasitoid Cotesia plutellae (Hymenoptera: Braconidae) on two host plant species. Bulletin of Entomological Research 93(1): 65-72.

Lof ME, Etienne RS, Powell J, de Gee M, Hemerik L. 2008. The effect of chemical information on the spatial distribution of fruit flies: I Model results. Bulletin of Mathematical Biology 70(7): 1827-1849.

Lommen A. 2009. MetAlign: interface-driven, versatile metabolomics tool for hyphenated full-scan mass spectrometry data preprocessing. Analytical Chemistry 81(8): 3079-3086.

Loreto F, Dicke M, Schnitzler J-P, Turlings TCJ. 2014. Plant volatiles and the environment. Plant, Cell \& Environment 37(8): 1905-1908.

Loughrin JH, Manukian A, Heath RR, Turlings TC, Tumlinson JH. 1994. Diurnal cycle of emission of induced volatile terpenoids by herbivore-injured cotton plant. Proceedings of the National Academy of Sciences 91(25): 11836-11840.

Lucas-Barbosa D, Poelman EH, Aartsma Y, Snoeren TAL, van Loon JJA, Dicke M. 2014. Caught between parasitoids and predators - survival of a specialist herbivore on leaves and flowers of mustard plants. Journal of Chemical Ecology 40(6): 621-631.

MacArthur RH, Pianka ER. 1966. On optimal use of a patchy environment. The American Naturalist 100(916): 603-609. 
Mallinger RE, Hogg DB, Gratton C. 2011. Methyl salicylate attracts natural enemies and reduces populations of soybean aphids (Hemiptera: Aphididae) in soybean agroecosystems. Journal of Economic Entomology 104(1): 115-124.

Mangiafico SS. 2018. rcompanion: Functions to support extension education program evaluation.

Manoukis NC, Hall B, Geib SM. 2014. A computer model of insect traps in a landscape. Scientific Reports 4: 7015.

Mäntylä E, Blande JD, Klemola T. 2014. Does application of methyl jasmonate to birch mimic herbivory and attract insectivorous birds in nature? Arthropod-Plant Interactions 8(2): 143-153.

Mäntylä E, Klemola T, Haukioja E. 2004. Attraction of willow warblers to sawfly-damaged mountain birches: novel function of inducible plant defences? Ecology Letters 7(10): 915-918.

Martin JP, Beyerlein A, Dacks AM, Reisenman CE, Riffell JA, Lei H, Hildebrand JG. 2011. The neurobiology of insect olfaction: Sensory processing in a comparative context. Progress in Neurobiology 95(3): 427-447.

Mattiacci L, Dicke M, Posthumus MA. 1995. beta-Glucosidase: an elicitor of herbivore-induced plant odor that attracts host-searching parasitic wasps. Proceedings of the National Academy of Sciences 92(6): 2036-2040.

Menalled FD, Costamagna AC, Marino PC, Landis DA. 2003. Temporal variation in the response of parasitoids to agricultural landscape structure. Agriculture, Ecosystems \& Environment 96(1): 29-35.

Milli R, Koch UT, de Kramer JJ. 1997. EAG measurement of pheromone distribution in apple orchards treated for mating disruption of Cydia pomonella. Entomologia Experimentalis Et Applicata 82(3): 289-297.

Mills NJ, Wajnberg E. 2008. Optimal foraging behaviour and efficient biological control methods. Behavioural Ecology of Insect Parasitoids-From Theoretical Approaches to Field Applications: 3-30.

Misztal PK 2016. Measuring rapid changes in plant volatiles at different spatial levels. In: Blande JD, Glinwood R (eds). Deciphering Chemical Language of Plant Communication. Cham: Springer International Publishing, 95-114.

Moraes MCB, Pareja M, Laumann RA, Hoffmann-Campo CB, Borges M. 2008. Response of the parasitoid Telenomus podisi to induced volatiles from soybean damaged by stink bug herbivory and oviposition. Journal of Plant Interactions 3(2): 111-118.

Moreira X, Abdala-Roberts L, Rasmann S, Castagneyrol B, Mooney KA. 2016. Plant diversity effects on insect herbivores and their natural enemies: current thinking, recent findings, and future directions. Current Opinion in Insect Science 14: 1-7.

Mumm R, Dicke M. 2010. Variation in natural plant products and the attraction of bodyguards involved in indirect plant defense. Canadian Journal of Zoology 88(7): 628-667.

Murakami M, Hirao T, Kasei A. 2008. Effects of habitat configuration on host-parasitoid food web structure. Ecological Research 23(6): 1039-1049.

Murlis J, Willis MA, Cardé RT. 2000. Spatial and temporal structures of pheromone plumes in fields and forests. Physiological Entomology 25(3): 211-222.

Murlis J, Elkinton JS, Cardé RT. 1992. Odor plumes and how insects use them. Annual Review of Entomology 37(1): 505-532.

Nakagawa S, Schielzeth H. 2012. A general and simple method for obtaining R2 from generalized linear mixed-effects models. Methods in Ecology and Evolution 4(2): 133-142.

Nathan R, Getz WM, Revilla E, Holyoak M, Kadmon R, Saltz D, Smouse PE. 2008. A movement ecology paradigm for unifying organismal movement research. Proceedings of the National Academy of Sciences 105(49): 19052-19059.

Ogle DH. 2018. FSA: Fisheries Stock Analysis.

Ohsaki N, Sato Y. 1999. The role of parasitoids in evolution of habitat and larval food plant preference by three Pieris butterflies. Researches on Population Ecology 41(1): 107-119.

Ohsaki N, Sato Y. 1994. Food plant choice of Pieris butterflies as a trade-off between parasitoid avoidance and quality of plants. Ecology 75(1): 59-68. 
Olden JD, Schooley RL, Monroe JB, Poff NL. 2004. Context-dependent perceptual ranges and their relevance to animal movements in landscapes. Journal of Animal Ecology 73(6): 1190-1194.

Papaj DR, Vet LEM. 1990. Odor learning and foraging success in the parasitoid, Leptopilina heterotoma. Journal of Chemical Ecology 16(11): 3137-3150.

Pareja M, Moraes MCB, Clark SJ, Birkett MA, Powell W. 2007. Response of the aphid parasitoid Aphidius funebris to volatiles from undamaged and aphid-infested Centaurea nigra. Journal of Chemical Ecology 33(4): 695-710.

PDOK 2016. TOPNL Historie, https://www.pdok.nl/nl/producten/pdok-downloads/basisregistratietopografie/topnl/topnl-historie.

Peñaflor MFGV, Gonçalves FG, Colepicolo C, Sanches PA, Bento JMS. 2017. Effects of single and multiple herbivory by host and non-host caterpillars on the attractiveness of herbivoreinduced volatiles of sugarcane to the generalist parasitoid Cotesia flavipes. Entomologia Experimentalis et Applicata 165(1): 83-93.

Penaflor MFGB, Bento JMS. 2013. Herbivore-induced plant volatiles to enhance biological control in agriculture. Neotropical Entomology 42(4): 331-343.

Perfecto I, Vet LEM. 2003. Effect of a nonhost plant on the location behavior of two parasitoids: The tritrophic system of Cotesia spp. (Hymenoptera: Braconidae), Pieris rapae (Lepidoptera: Pieridae), and Brassica oleraceae. Environmental Entomology 32(1): 163-174.

Petermann JS, Müller CB, Weigelt, Alexandra, Weisser WW, Schmid B. 2010. Effect of plant species loss on aphid-parasitoid communities. Journal of Animal Ecology 79(3): 709-720.

Plećaš M, Gagić V, Janković M, Petrović-Obradović O, Kavallieratos NG, Tomanović Z, Thies C, Tscharntke T, Ćetković A. 2014. Landscape composition and configuration influence cereal aphid-parasitoid-hyperparasitoid interactions and biological control differentially across years. Agriculture, Ecosystems \& Environment 183: 1-10.

Poelman EH, Kos M 2016. Complexity of plant volatile-mediated interactions beyond the third trophic level. Deciphering Chemical Language of Plant Communication: Springer, 211-225.

Poelman EH. 2015. From induced resistance to defence in plant-insect interactions. Entomologia Experimentalis et Applicata 157(1): 11-17.

Poelman EH, Harvey JA, van Loon JJA, Vet LEM, Dicke M. 2013. Variation in herbivore-induced plant volatiles corresponds with spatial heterogeneity in the level of parasitoid competition and parasitoid exposure to hyperparasitism. Functional Ecology 27(5): 1107-1116.

Poelman EH, van Loon JJA, van Dam NM, Vet LEM, Dicke M. 2010. Herbivore-induced plant responses in Brassica oleracea prevail over effects of constitutive resistance and result in enhanced herbivore attack. Ecological Entomology 35(2): 240-247.

Poelman EH, van Dam NM, van Loon JJA, Vet LEM, Dicke M. 2009a. Chemical diversity in Brassica oleracea affects biodiversity of insect herbivores. Ecology 90(7): 1863-1877.

Poelman EH, Oduor AMO, Broekgaarden C, Hordijk CA, Jansen JJ, van Loon JJA, van Dam NM, Vet LEM, Dicke M. 2009b. Field parasitism rates of caterpillars on Brassica oleracea plants are reliably predicted by differential attraction of Cotesia parasitoids. Functional Ecology 23(5): 951-962.

Pollard KA, Holland JM. 2006. Arthropods within the woody element of hedgerows and their distribution pattern. Agricultural and Forest Entomology 8(3): 203-211.

Ponzio C, Cascone P, Cusumano A, Weldegergis BT, Fatouros NE, Guerrieri E, Dicke M, Gols R. 2016. Volatile-mediated foraging behaviour of three parasitoid species under conditions of dual insect herbivore attack. Animal Behaviour 111: 197-206.

Potting RPJ, Vet LEM, Dicke M. 1995. Host microhabitat location by stem-borer parasitoid Cotesia flavipes: the role of herbivore volatiles and locally and systemically induced plant volatiles. Journal of Chemical Ecology 21(5): 525-539.

Potts SG, Biesmeijer JC, Kremen C, Neumann P, Schweiger O, Kunin WE. 2010. Global pollinator declines: trends, impacts and drivers. Trends in Ecology \& Evolution 25(6): 345-353.

Poveda K, Martínez E, Kersch-Becker Monica F, Bonilla Maria A, Tscharntke T. 2012. Landscape simplification and altitude affect biodiversity, herbivory and Andean potato yield. Journal of Applied Ecology 49(2): 513-522. 
Powell W, Pennacchio F, Poppy GM, Tremblay E. 1998. Strategies Involved in the Location of Hosts by the Parasitoid Aphidius ervi Haliday (Hymenoptera: Braconidae: Aphidiinae). Biological Control 11(2): 104-112.

Price PW, Bouton CE, Gross P, McPheron BA, Thompson JN, Weis AE. 1980. Interactions among three trophic levels: influence of plants on interactions between insect herbivores and natural enemies. Annual review of Ecology and Systematics 11(1): 41-65.

Puente M, Magori K, Kennedy GG, Gould F. 2008. Impact of herbivore-induced plant volatiles on parasitoid foraging success: A spatial simulation of the Cotesia rubecula, Pieris rapae, and Brassica oleracea system. Journal of Chemical Ecology 34(7): 959-970.

Raguso RA, Agrawal AA, Douglas AE, Jander G, Kessler A, Poveda K, Thaler JS. 2015. The raison d'être of chemical ecology. Ecology 96(3): 617-630.

Ramsden M, Menendez R, Leather S, Wäckers F. 2016. Do natural enemies really make a difference? Field scale impacts of parasitoid wasps and hoverfly larvae on cereal aphid populations. Agricultural and Forest Entomology 10.1111/afe.12191.

Randlkofer B, Obermaier E, Casas J, Meiners T. 2010a. Connectivity counts: disentangling effects of vegetation structure elements on the searching movement of a parasitoid. Ecological Entomology 35(4): 446-455.

Randlkofer B, Obermaier E, Hilker M, Meiners T. 2010b. Vegetation complexity-The influence of plant species diversity and plant structures on plant chemical complexity and arthropods. Basic and Applied Ecology 11(5): 383-395.

Rasmann S, Köllner TG, Degenhardt J, Hiltpold I, Toepfer S, Kuhlmann U, Gershenzon J, Turlings TC. 2005. Recruitment of entomopathogenic nematodes by insect-damaged maize roots. Nature 434(7034): 732-737.

Reinecke A, Hilker M. 2014. Plant semiochemicals - perception and behavioural responses by insects. Annual Plant Reviews volume 47: Insect-Plant Interactions 10.1002/9781118829783. ch4: 115-153.

Rezende MQ, Venzon M, Perez AL, Cardoso IM, Janssen A. 2014. Extrafloral nectaries of associated trees can enhance natural pest control. Agriculture, Ecosystems \& Environment 188: 198-203.

Riffell JA, Abrell L, Hildebrand JG. 2008. Physical processes and real-time chemical measurement of the insect olfactory environment. Journal of Chemical Ecology 34(7): 837-853.

Rodriguez-Saona C, Kaplan I, Braasch J, Chinnasamy D, Williams L. 2011. Field responses of predaceous arthropods to methyl salicylate: A meta-analysis and case study in cranberries. Biological Control 59(2): 294-303.

Roitberg BD, Gillespie DR. 2014. Natural enemies on the landscape - Integrating life-history theory and landscapes. Biological Control 75: 39-47.

Roland J, Taylor PD. 1997. Insect parasitoid species respond to forest structure at different spatial scales. Nature 386(6626): 710-713.

Rowen E, Kaplan I. 2016. Eco-evolutionary factors drive induced plant volatiles: a meta-analysis. New Phytologist 210(1): 284-294.

Rusch A, Chaplin-Kramer R, Gardiner MM, Hawro V, Holland J, Landis D, Thies C, Tscharntke T, Weisser WW, Winqvist C, et al. 2016. Agricultural landscape simplification reduces natural pest control: A quantitative synthesis. Agriculture, Ecosystems \& Environment 221: 198-204.

Rusch A, Valantin-Morison M, Sarthou JP, Roger-Estrade J 2010. Biological control of insect pests in agroecosystems: effects of crop management, farming systems, and seminatural habitat at the landscape scale: a review. In: Sparks DL ed. Advances in Agronomy, Vol 109. San Diego: Elsevier Academic Press Inc, 219-259.

Scascighini N, Mattiacci L, D'Alessandro M, Hern A, Sybille Rott A, Dorn S. 2005. New insights in analysing parasitoid attracting synomones: early volatile emission and use of stir bar sorptive extraction. Chemoecology 15(2): 97-104.

Schellhorn NA, Gagic V, Bommarco R. 2015. Time will tell: resource continuity bolsters ecosystem services. Trends in Ecology \& Evolution 30(9): 524-530.

Schellhorn NA, Bianchi F, Hsu CL. 2014. Movement of entomophagous arthropods in agricultural landscapes: Links to pest suppression. Annual Review of Entomology 59: 559-581. 
Schoonhoven LM, van Loon JJA, Dicke M. 2005. Insect-Plant Biology. Oxford: Oxford University Press.

Schröder R, Hilker M. 2008. The relevance of background odor in resource location by insects: A behavioral approach. BioScience 58(4): 308-316.

Shiojiri K, Ozawa R, Kugimiya S, Uefune M, van Wijk M, Sabelis MW, Takabayashi J. 2010. Herbivorespecific, density-dependent induction of plant volatiles: honest or "cry wolf" signals? PLOS ONE 5(8): e12161.

Shiojiri K, Takabayashi J, Yano S, Takafuji A. 2000. Flight response of parasitoids toward plantherbivore complexes: A comparative study of two parasitoid-herbivore systems on cabbage plants. Applied Entomology and Zoology 35(1): 87-92.

Sih A, Cote J, Evans M, Fogarty S, Pruitt J. 2012. Ecological implications of behavioural syndromes. Ecology Letters 15(3): 278-289.

Šimpraga M, Takabayashi J, Holopainen JK. 2016. Language of plants: Where is the word? Journal of Integrative Plant Biology 58(4): 343-349.

Simpson M, Gurr GM, Simmons AT, Wratten SD, James DG, Leeson G, Nicol HI, Orre-Gordon GUS. 2011. Attract and reward: combining chemical ecology and habitat manipulation to enhance biological control in field crops. Journal of Applied Ecology 48(3): 580-590.

Smallegange R, van Loon J, Blatt S, A. Harvey J, Dicke M. 2008. Parasitoid load affects plant fitness in a tritrophic system Entomologia Experimentalis et Applicata 128:172-183.

Smid HM, Vet LEM. 2016. The complexity of learning, memory and neural processes in an evolutionary ecological context. Current Opinion in Insect Science 15: 61-69.

Smid HM, Wang G, Bukovinszky T, Steidle JLM, Bleeker MAK, van Loon JJA, Vet LEM. 2007. Speciesspecific acquisition and consolidation of long-term memory in parasitic wasps. Proceedings of the Royal Society B: Biological Sciences 274(1617): 1539.

Smilanich AM, Dyer LA, Chambers JQ, Bowers MD. 2009. Immunological cost of chemical defence and the evolution of herbivore diet breadth. Ecology Letters 12(7): 612-621.

Snoeren TA, Mumm R, Poelman EH, Yang Y, Pichersky E, Dicke M. 2010. The herbivore-induced plant volatile methyl salicylate negatively affects attraction of the parasitoid Diadegma semiclausum. Journal of Chemical Ecology 36(5): 479-489.

Soler R, Harvey JA, Bezemer TM. 2007. Foraging efficiency of a parasitoid of a leaf herbivore is influenced by root herbivory on neighbouring plants. Functional Ecology 21(5): 969-974.

Stam JM, Kroes A, Li Y, Gols R, van Loon JJA, Poelman EH, Dicke M. 2014. Plant interactions with multiple insect herbivores: from community to genes. Annual Review of Plant Biology 65(1): 689-713.

Stenberg JA, Heil M, Åhman I, Björkman C. 2015. Optimizing crops for biocontrol of pests and disease. Trends in Plant Science 20(11): 698-712.

Stork WF, Weinhold A, Baldwin IT. 2011. Trichomes as dangerous lollipops: Do lizards also use caterpillar body and frass odor to optimize their foraging? Plant signaling \& Behavior 6(12): 1893-1896.

Strauss SY, Sahli H, Conner JK. 2004. Toward a more trait-centered approach to diffuse (co)evolution. New Phytologist 165(1): 81-90.

Suzuki R, Shimodaira H. 2015. pvclust: Hierarchical clustering with p-values via multiscale bootstrap resampling.

Suzuki R, Shimodaira H. 2006. pvclust: an R package for assessing the uncertainty in hierarchical clustering. Bioinformatics 22(12): 1540-1542.

Takabayashi J, Takahashi S. 1990. An allelochemical elicits arrestment in Apanteles kariyai in feces of nonhost larvae Acantholeucania loreyi. Journal of Chemical Ecology 16(6): 2009-2017.

Tentelier C, Fauvergue X. 2007. Herbivore-induced plant volatiles as cues for habitat assessment by a foraging parasitoid. Journal of Animal Ecology 76(1): 1-8.

Thiel A, Hoffmeister TS. 2009. Decision-making dynamics in parasitoids of Drosophila. Advances in Parasitology Volume 70: 45-66.

Thies C, Steffan-Dewenter I, Tscharntke T. 2003. Effects of landscape context on herbivory and parasitism at different spatial scales. Oikos 101(1): 18-25. 
Tikunov YM, Laptenok S, Hall RD, Bovy A, de Vos RC. 2012. MSClust: a tool for unsupervised mass spectra extraction of chromatography-mass spectrometry ion-wise aligned data. Metabolomics 8(4): 714-718.

Tscharntke T, Karp DS, Chaplin-Kramer R, Batáry P, DeClerck F, Gratton C, Hunt L, Ives A, Jonsson $M$, Larsen A, et al. 2016. When natural habitat fails to enhance biological pest control - Five hypotheses. Biological Conservation 204: 449-458.

Tscharntke T, Tylianakis JM, Rand TA, Didham RK, Fahrig L, Batary P, Bengtsson J, Clough Y, Crist TO, Dormann CF. 2012. Landscape moderation of biodiversity patterns and processes-eight hypotheses. Biological Reviews 87(3): 661-685.

Tscharntke T, Bommarco R, Clough Y, Crist TO, Kleijn D, Rand TA, Tylianakis JM, Nouhuys Sv, Vidal S. 2007. Conservation biological control and enemy diversity on a landscape scale. Biological Control 43(3): 294-309.

Tscharntke T, Klein AM, Kruess A, Steffan-Dewenter I, Thies C. 2005a. Landscape perspectives on agricultural intensification and biodiversity - ecosystem service management. Ecology Letters 8(8): 857-874.

Tscharntke T, Rand TA, Bianchi FJ 2005b. The landscape context of trophic interactions: insect spillover across the crop-noncrop interface. Annales Zoologici Fennici 42: 421-432.

Tscharntke T, Brandl R. 2004. Plant-insect interactions in fragmented landscapes. Annual Review of Entomology 49(1): 405-430.

Turlings TCJ, Erb M. 2018. Tritrophic interactions mediated by herbivore-induced plant volatiles: Mechanisms, ecological relevance, and application potential. Annual Review of Entomology 63(1): 433-452.

Turlings TCJ, Bernasconi M, Bertossa R, Bigler F, Caloz G, Dorn S. 1998. The induction of volatile emissions in maize by three herbivore species with different feeding habits: Possible consequences for their natural enemies. Biological Control 11(2): 122-129.

Turlings TCJ, Tumlinson JH, Lewis WJ. 1990. Exploitation of herbivore-induced plant odors by hostseeking parasitic wasps. Science 250(4985): 1251-1253.

Uefune M, Kugimiya S, Sano K, Takabayashi J. 2012. Herbivore-induced plant volatiles enhance the ability of parasitic wasps to find hosts on a plant. Journal of Applied Entomology 136(1-2): 133-138.

Utsumi S, Ando Y, Miki T. 2010. Linkages among trait-mediated indirect effects: a new framework for the indirect interaction web. Population Ecology 52(4): 485-497.

van Alphen JJM, Bernstein C, Driessen G. 2003. Information acquisition and time allocation in insect parasitoids. Trends in Ecology \& Evolution 18(2): 81-87.

van den Boom CEM, van Beek TA, Posthumus MA, de Groot A, Dicke M. 2004. Qualitative and quantitative variation among volatile profiles induced by Tetranychus urticae feeding on plants from various families. Journal of Chemical Ecology 30(1): 69-89.

van Nouhuys S. 2005. Effects of habitat fragmentation at different trophic levels in insect communities. Annales Zoologici Fennici 42(4): 433-447.

van Nouhuys S, Ehrnsten J. 2004. Wasp behavior leads to uniform parasitism of a host available only a few hours per year. Behavioral Ecology 15(4): 661-665.

van Nouhuys S, Hanski I. 2002. Colonization rates and distances of a host butterfly and two specific parasitoids in a fragmented landscape. Journal of Animal Ecology 71(4): 639-650.

van Wijk M, de Bruijn PJA, Sabelis MW. 2011. Complex odor from plants under attack: Herbivore's enemies react to the whole, not its parts. PLOS ONE 6(7): e21742.

Vet LEM. 2001. Parasitoid searching efficiency links behaviour to population processes. Applied Entomology and Zoology 36(4): 399-408.

Vet LEM, de Jong AG, Franchi E, Papaj DR. 1998. The effect of complete versus incomplete information on odour discrimination in a parasitic wasp. Animal Behaviour 55(5): 1271-1279.

Vet LEM, Dicke M. 1992. Ecology of infochemical use by natural enemies in a tritrophic context. Annual Review of Entomology 37(1): 141-172.

Vinatier F, Tixier P, Duyck P-F, Lescourret F. 2011a. Factors and mechanisms explaining spatial heterogeneity: a review of methods for insect populations. Methods in Ecology and Evolution 2(1): 11-22. 
Vinatier F, Lescourret F, Duyck P-F, Martin O, Senoussi R, Tixier P. 2011b. Should I stay or should I go? A habitat-dependent dispersal kernel improves prediction of movement. PLOS ONE 6(7): e21115.

Vinson SB. 1976. Host selection by insect parasitoids. Annual Review of Entomology 21(1): 109-133.

Völkl W. 2000. Foraging behaviour and sequential multisensory orientation in the aphid parasitoid, Pauesia picta (Hym., Aphidiidae) at different spatial scales. Journal of Applied Entomology 124(7-8): 307-314.

Vollhardt IMG, Tscharntke T, Wäckers FL, Bianchi FJJA, Thies C. 2008. Diversity of cereal aphid parasitoids in simple and complex landscapes. Agriculture, Ecosystems \& Environment 126(34): 289-292.

von Mérey GE, Veyrat N, Lange Ed, Degen T, Mahuku G, Valdez RL, Turlings TCJ, D’Alessandro M. 2012. Minor effects of two elicitors of insect and pathogen resistance on volatile emissions and parasitism of Spodoptera frugiperda in Mexican maize fields. Biological Control 60(1): 7-15.

Vos M, Vet LEM, Wäckers FL, Middelburg JJ, van der Putten WH, Mooij WM, Heip CHR, van Donk E. 2006. Infochemicals structure marine, terrestrial and freshwater food webs: Implications for ecological informatics. Ecological Informatics 1(1): 23-32.

Vos M, Berrocal SM, Karamaouna F, Hemerik L, Vet LEM. 2001. Plant-mediated indirect effects and the persistence of parasitoid-herbivore communities. Ecology Letters 4(1): 38-45.

Vos M, Hemerik L, Vet Louise EM. 1998. Patch exploitation by the parasitoids Cotesia rubecula and Cotesia glomerata in multi-patch environments with different host distributions. Journal of Animal Ecology 67(5): 774-783.

Voskamp K, Den Otter CJ, Noorman N. 1998. Electroantennogram responses of tsetse flies (Glossina pallidipes) to host odours in an open field and riverine woodland. Physiological Entomology 23(2): 176-183.

Wäckers FL. 2001. A comparison of nectar- and honeydew sugars with respect to their utilization by the hymenopteran parasitoid Cotesia glomerata. Journal of Insect Physiology 47(9): 10771084.

Wajnberg É. 2006. Time allocation strategies in insect parasitoids: from ultimate predictions to proximate behavioral mechanisms. Behavioral Ecology and Sociobiology 60(5): 589-611.

Wanner H, Gu H, Dorn S. 2006. Nutritional value of floral nectar sources for flight in the parasitoid wasp, Cotesia glomerata. Physiological Entomology 31(2): 127-133.

Wäschke N, Hardge K, Hancock C, Hilker M, Obermaier E, Meiners T. 2014. Habitats as complex odour environments: How does plant diversity affect herbivore and parasitoid orientation? PLOS ONE 9(1): e85152

Webster B, Cardé RT. 2017. Use of habitat odour by host-seeking insects. Biological Reviews 92: 1241-1249

Werner EE, Peacor SD. 2003. A review of trait-mediated indirect interactions in ecological communities. Ecology 84(5): 1083-1100.

Wickham H. 2009. ggplot2: Elegant graphics for data analysis: Springer-Verlag New York.

Williams IH, Frearson DJ, Barari H, McCartney A. 2007. First field evidence that parasitoids use upwind anemotaxis for host-habitat location. Entomologia Experimentalis et Applicata 123(3): 299-307.

Willis MA, Murlis J, Cardé RT. 1991. Pheromone-mediated upwind flight of male gypsy moths, Lymantria dispar, in a forest. Physiological Entomology 16(4): 507-521.

Winkler K, Wäckers F, Pinto DM. 2009. Nectar-providing plants enhance the energetic state of herbivores as well as their parasitoids under field conditions. Ecological Entomology 34(2): 221-227.

Wissinger SA. 1997. Cyclic colonization in predictably ephemeral habitats: A template for biological control in annual crop systems. Biological Control 10(1): 4-15.

Woltz JM, Isaacs R, Landis DA. 2012. Landscape structure and habitat management differentially influence insect natural enemies in an agricultural landscape. Agriculture Ecosystems \& Environment 152: 40-49. 
Wyckhuys KAG, Lu YH, Morales H, Vazquez LL, Legaspi JC, Eliopoulos PA, Hernandez LM. 2013. Current status and potential of conservation biological control for agriculture in the developing world. Biological Control 65(1): 152-167.

Zhou K, Huang J, Deng X, van der Werf W, Zhang W, Lu Y, Wu K, Wu F. 2014. Effects of land use and insecticides on natural enemies of aphids in cotton: First evidence from smallholder agriculture in the North China Plain. Agriculture, Ecosystems \& Environment 183: 176-184.

Zhu F, Broekgaarden C, Weldegergis BT, Harvey JA, Vosman B, Dicke M, Poelman EH. 2015. Parasitism overrides herbivore identity allowing hyperparasitoids to locate their parasitoid host using herbivore-induced plant volatiles. Molecular Ecology 24(11): 2886-2899.

Zimmer RK, Zimmer CA. 2008. Dynamic scaling in chemical ecology. Journal of Chemical Ecology 34(7): 822-836.

Zollner PA, Lima SL. 2005. Behavioral tradeoffs when dispersing across a patchy landscape. Oikos 108(2): 219-230.

Zuur A, leno EN, Walker N, Saveliev AA, Smith GM. 2009. Mixed Effects Models and Extensions in Ecology with R: Springer New York. 


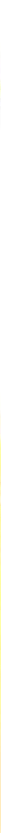




\section{Summary}


Plants need to defend themselves from attack by herbivorous insects. They can do this directly by producing chemical and structural defences such as toxins and spines, but also indirectly by promoting the effectiveness of enemies of the herbivores. This can be accomplished by providing resources such as nectar or shelter for these enemies, but also by providing information to the enemy on the location of their prey. These interactions form a mechanistic basis of tritrophic interactions: Interactions between plants, herbivores and enemies of these herbivores. Plants can provide natural enemies with information on herbivores by releasing chemical compounds upon damage inflicted by these herbivores. These chemical compounds are called herbivore-induced plant volatiles (HIPVs) and can be used by carnivorous animals to find their prey. Some plant species or varieties produce different HIPV blends than others, which can influence carnivore preference such as parasitic wasps or parasitoids.

Parasitoids are insects that lay their eggs in or on other insects. Their offspring feed from the host insect until the parasitoid completes larval development, which usually results in the death of the host. After emerging as adults and mating, female parasitoids need to find new hosts in their environment. These hosts can be spread heterogeneously, which means the parasitoid needs to disperse to locate the hosts. While foraging for hosts, parasitoids can use HIPVs as information on the identity, quality and abundance of hosts. This process has been well characterized at small spatial scales, but little research has been done on how HIPVs attract parasitoids at larger spatial scales. The spatial distribution of HIPVs can be influenced by a range of aspects of the surrounding environment, such as weather conditions, vegetation structure and interference with chemical compounds from other plants. Little is known on how these habitat characteristics affect the foraging behaviour of parasitoids under field conditions. Furthermore, research that combines plant variation in attractiveness to parasitoids with the effects of habitat characteristics is rare.

The aim of this thesis project was to investigate HIPV-mediated interactions between plants and parasitoids from local to landscape scale, and how plant variation in attraction of parasitoids via HIPVs alters parasitoid foraging behaviour at these spatial scales. I used a tritrophic system of white cabbage, Pieris brassicae 
(large cabbage white butterfly) and the parasitic wasp Cotesia glomerata. Two cabbage accessions were used, which were a priori known to differ in attractiveness to parasitoids under laboratory conditions and in small-scale field experiments. The accessions Christmas Drumhead is preferred over Badger Shipper.

In Chapter 2, the current state of the literature regarding HIPV-mediated interactions across spatial scales is reviewed. Key knowledge gaps in the use of HIPVs as a long-distance cue by parasitoids are the distance from which they can be perceived, how HIPVs from surrounding vegetation alter the ability of a parasitoid to find their hosts and how parasitoids use HIPV s on the landscape scale.

The spatial scale of parasitoid attraction by two plant accessions that differ in attractiveness was studied in Chapter 3. In an open field experiment, I released parasitoids in an experimental set-up with cabbage plants infested with caterpillars either at a spacing of $10 \mathrm{~m}$ or of $20 \mathrm{~m}$. Parasitoids which were released in set-ups with the accession Christmas Drumhead parasitized caterpillars at a similar rate in set-ups of $10 \mathrm{~m}$ and $20 \mathrm{~m}$. In set-ups with the less attractive accession, Badger Shipper, parasitism rates decreased dramatically when distance between plants was increased from $10 \mathrm{~m}$ to $20 \mathrm{~m}$. Additionally, detailed parasitoid behaviour was studied on a smaller scale (up to $8 \mathrm{~m}$ ) in a semifield set-up (tent). Similarly, I found that parasitoids are less able to find plants at larger distances, but that the more attractive accession Christmas Drumhead was found more frequently than the less attractive accession Badger Shipper at larger distances. The experiments show that parasitoids can be attracted to herbivore-infested plants over distances between 10 and $20 \mathrm{~m}$, and that a more attractive host-infested variety is found by parasitoids over longer distances. Habitat characteristics can influence parasitoid foraging behaviour. More specifically, the number of plants in a patch might affect the apparency of this patch to parasitoids, but also to herbivores, and the presence of another plant species might influence the ability of parasitoids and herbivores to find their food plant. In another field experiment (Chapter 4), I established cabbage plots of the two accessions which varied in plot size (small or large) and which had either no border, or a border of black mustard plants, Brassica nigra, a close relative of cabbage. Throughout the season, I investigated whether 
experimentally introduced Pieris brassicae caterpillars were parasitized and counted naturally occurring Pieris spp caterpillars in the plots. Abundance of the caterpillar Pieris rapae was not affected by cabbage accession or plot size, and only later in the season fewer caterpillars were found in bordered plots than non-bordered plots. Parasitism rates of experimentally introduced caterpillars were also not affected by plot size. The border only affected parasitism rates on the less attractive accession Badger Shipper, where fewer caterpillars were parasitized. The more attractive accession Christmas Drumhead had equal parasitism rates in plots with or without a border. This accession also had higher incidence of superparasitism (where the same or multiple Cotesia glomerata parasitized a caterpillar multiple times). Results show that herbivores and parasitoids responded differently to variation in habitat characteristics and plant accession, which might alter the outcomes of tritrophic interactions on longer timescales. Accessions less apparent to parasitoids might provide herbivores with a refuge space, where parasitism risk is lower. For parasitoids, more apparent plants may be easier to find in complex vegetation structure.

Parasitoid foraging behaviour may be affected when other insects, which are not hosts (non-hosts), are feeding from the same or neighbouring plants and alter HIPV emissions from these plants. In Chapter 5, I studied parasitoid foraging behaviour in environments with different distributions of plant and herbivores on these plants. In a semi-field tent set-up, plants of both accessions were mixed and infested with hosts or hosts and non-hosts. In most combinations of host or non-host caterpillars on the plants, the more attractive accession Christmas Drumhead had higher parasitism rates than Badger Shipper. However, when both hosts and non-hosts were present on Badger Shipper and only hosts on Christmas Drumhead, overall parasitism rates in the tent decreased. In a wind tunnel experiment, parasitoid preference was studied in more detail. The accession Christmas Drumhead was overall the preferred accession, but some combinations of host and non-host infestation led to disappearance of this preference. In a third experiment, blends of volatile organic compounds were collected from the plants through headspace sampling and chemically analysed. HIPV blends different between accessions. However, these differences did not fully explain the findings of the complex semi-field experiment. 
Nevertheless, I identified that plant trait variation in HIPVs intricately interacts with non-host presence in its effect on parasitoid host-location efficiency.

Finally, in Chapter 6, I investigated how parasitism rates are affected by landscape context and how plants which differ in attraction of parasitoids are affected differently by these aspects of the landscape. In a field experiment, I placed cabbage plants of the two accessions in 19 different landscapes in the vicinity of Wageningen, the Netherlands. On these cabbage plants, I assessed parasitism rates of caterpillars by the naturally occurring parasitoid population. Additionally, I measured landscape characteristics such as the area of arable land, pastures, forest and non-woody seminatural area. Furthermore, a more functional landscape characteristic was quantified, the cover of plants from the family Brassicaceae (the food plant family of the host herbivore, P. brassicae). Parasitism rates were positively associated with the area of (mostly organic) arable land and brassicaceous plant cover, but this effect was larger for the more attractive accession Christmas Drumhead than for the less attractive accession Badger Shipper. The area of forest in the landscape was negatively associated with parasitism rates, which can be explained by the forest's relatively low abundance of brassicaceous plants. I conclude that a more attractive accession is able to attract more parasitoids when there are sufficiently sized parasitoid populations nearby in the landscape by having a larger attraction radius. Additionally, for tritrophic interactions in which specialist insects are involved, functional characteristics of the landscape such as cover of host plants of the herbivore hosts of the parasitoid, can be more useful to explain parasitism rates than land use classes.

This thesis makes a contribution to the fundamental knowledge of foraging behaviour in complex field situations and, therefore, the relevance of HIPVs in mediating tritrophic interactions in natural and agricultural systems. In Chapter 7, I discuss my findings in a broader context. HIPVs are important longdistance cues for parasitoids to find their hosts in complex situations, where plants with hosts are patchily distributed with varying distance, embedded in vegetation structure. The connection between different plant patches can be strengthened by HIPVs. However, whether a higher attractiveness to parasitoids via HIPVs leads to higher parasitism rates can depend on characteristics of the 
plant's habitat. This is interesting for HIPVs as a resistance or defence trait. The production of HIPVs can be ecologically costly when they also 'advertise' the plant to other herbivores or to hyperparasitoids which parasitize parasitoids. In situations where a higher HIPV attractiveness does not lead to higher parasitism rates, producing these HIPV blends does not give an advantage over plants which do not produce them. However, the relevance of HIPVs for plant fitness of these plants in different situations remains to be investigated. I also discuss the use of plant varieties with increased attractiveness to parasitoids in agriculture. Plants could be specifically bred for increased attractiveness over larger distance and in more complex situations. However, the landscape context is an important factor influencing natural enemy populations from which these natural enemies can be attracted to crop fields. It is, therefore, important to also consider resource needs of natural enemies and connectivity from stable populations to fields where enemies are needed to suppress pest populations. Because in highly simplified landscapes fragments with stable enemy populations are scattered and have low connectivity with other fragments or agricultural fields were enemies are needed, I conclude that conservation biological control measures should be implemented on a regional scale. Additionally, for more attractive varieties to be marketable to (organic) farmers, they need to produce a higher yield than less attractive varieties, something which yet has to be studied. Finally, how precisely HIPVs from different plants disperse through the environment has yet to be determined, which is methodologically challenging. Also, different parasitoid species may respond differently to the habitat characteristics used in this study to investigate parasitoid behaviour. Studying parasitoid traits in relation to its response to volatiles might give a better understanding of the mechanisms of foraging behaviour via HIPVs in the field. In conclusion, this thesis provides important insights in the role of HIPVs at spatial scales ranging from the local scale to the landscape scale. Such information is important for developing sustainable crop protection under field conditions. 


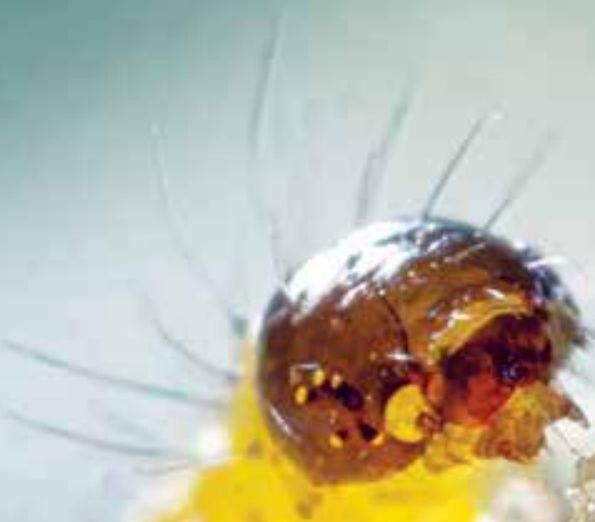

- 8 (5)

itaris

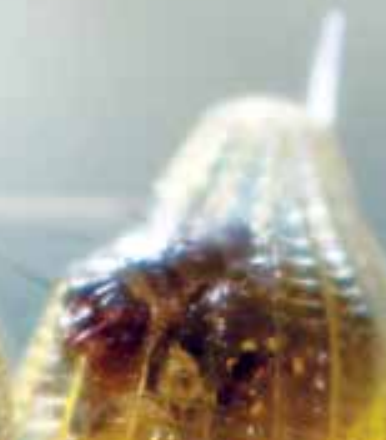

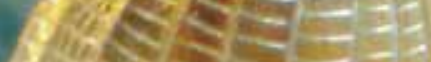
I.
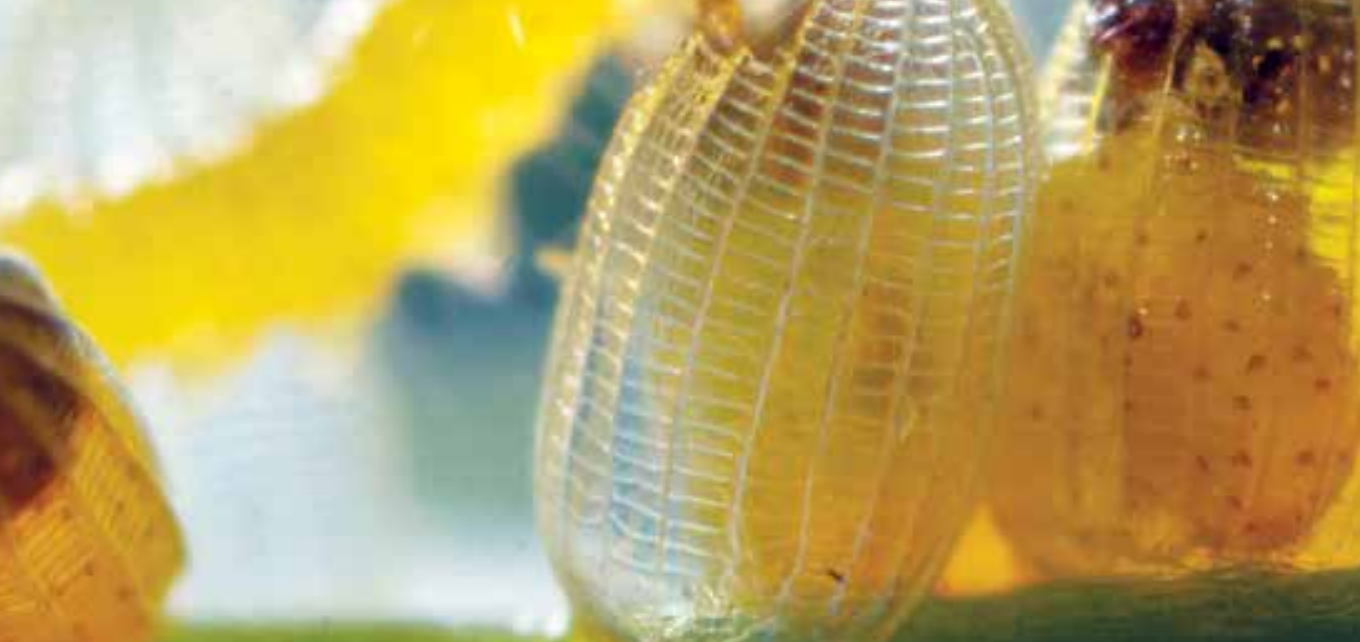

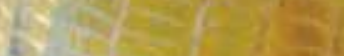

t.

$x^{6}=1$

, 


\section{Acknowledgements}


It seems like yesterday that I started this project, but in fact it is already five years ago that I applied! Now that this life chapter is coming to an end, it's time for me to reflect and think of all the support I had in these years. I had the benefit of working on my $\mathrm{PhD}$ project in a very supporting 'landscape', where many individuals interacted with me, directly and indirectly influencing my work! Although I believe that words cannot describe how grateful I am, I will make an attempt to acknowledge all of you in this section.

Ik dank al mijn supervisors voor het vertrouwen in mij en mijn project. Marcel, bedankt voor de goede begeleiding! Je had altijd snelle feedback, zelfs terwijl je met sabbatical was toen de inleverdatum wel erg dichtbij kwam. Felix, wat leuk dat ik je eerste $\mathrm{PhD}$ mag zijn die haar thesis mag verdedigen! Ik kon altijd jouw kantoor binnenlopen voor een kort (of lang) gesprek. Je luisterde aandachtig naar mijn soms wel heel erg uitgebreide uitleg over analyses en kleine details die ik had gevonden maar die toch niet zo heel belangrijk waren. Erik, je bent echt een voorbeeld van een goede supervisor. Je bent een echte mentor en hebt met motiverende woorden en reflecterende stiltes mij door dit project heen geleid. Bedankt voor de kansen die je me hebt gegeven en voor alles wat ik van jou heb geleerd. Wopke, ook al zijn we niet aan modelleren toe gekomen, ik ben heel dankbaar voor je bijdragen aan de statistiek. Ook waardeer ik het erg dat ondanks dat je vaak op reis was, je toch tijd maakte om al mijn veldexperimenten een keer te bezoeken. Je bent volgens mij de enige van de vier die dat gedaan heeft;)

The work in this thesis could not have been done without the support of a number of people involved in supplying the plants and insects, and taking care of the field. Therefore, I wish to thank Léon, Frans, André, Joop W and Pieter for the rearing of the insects used in this work. I also thank John, Gerard, Rinie and the many others from Unifarm who grew the plants and managed the field. Thank you Dine, for your help with my first field experiment and being my guardian angel in times of emergencies. Alexandre, thank you for the collaboration on the volatile analysis in the last year! I have learned a lot while working with you, and I appreciate the work we did together to make sure that the results could be included in the thesis chapter. 
Besides staff support, I also had help from several students in the data collection for the chapters of this thesis. Benjamin, you were my first student, but as you were doing your second MSc programme already, I always felt we were on the same level. Silvia, I greatly enjoyed spending time with you in the field and your curiosity and willingness to learn and discover new things. Kimmy, although your project was difficult and you faced several experimental problems, you handled the resulting data well and reported them in a very organised way. Yueyi, you were a great help in the last large field experiment, where we spent many hours together in the car talking about anything. Miguél, although I was not your official supervisor, you still contributed to one of the chapters in this thesis. You are very enthusiastic about the work and have bugged (;-)) me a lot over the years about when the paper is going to be published. I hope to make that wish come true soon!

Maite and Pulu! Thank you very much for agreeing to be my paranymphs and supporting me on the day of my defence! Pulu, we met during our MSc thesis and I'm happy we continued seeing each other throughout the years. Maite, whenever I talk with you I leave the conversation with more energy and enthusiasm than before. When I think I can't do it, you convince me I can.

Dani, when you were my EABI supervisor and later my MSc thesis supervisor, the idea of doing a PhD leaked into my mind. Your enthusiasm about scientific research is contagious and I'm happy you were at Ento for some more time after your own $\mathrm{PhD}$. I'm also happy that I have at least one person to share my wasp stories and pictures with! Social wasps are awesome! Lucille, thank you for the many way-too-long talks we had during work hours, even when we actually did not have time for them. You are truly a unique person. I am also very happy you invited us to your hometown after the SIP. It was a great trip and I will cherish the memories of that place.

Over the years I've felt very much a part of the 'Ento family', and all the activities we did together. Lucille, Quint and Tim, organising the labuitje together was a lot of fun! I also really enjoyed organising activities as member of the party committee. Sandeep, Yidong, Margot, Maite, Jeroen, Kay, Jessica and Stijn, 
thank you for the many lunch meetings and crazy ideas we sometimes had, and of course all the posters you let me create. Also many thanks to everyone in Erik's team: Feng, Marjolein, Jeltje, Jorad, Martin, Quint, Daan, Ilka, Maite, Antonio, Janneke and Klaas. Dinners, celebrating during meetings and brainstorming together were always enjoyable, and even led to writing a review with some of you! Peter, het was altijd lachen geblazen bij het Insect Ecology practicum met jou en Erik. Jeroen, wat leuk dat ik je thesis mocht opmaken! Het was een goede oefening en heeft me erg geholpen om dit boek te maken. Over the years I have met many interesting and cool Entomologists! I don't think I'll manage to mention everyone, but I also thank Karol, Emma, Jitte, Camille, Cindy, Shuhang, Foteini, Julia, Gerard, Chantal, Dennis, Peter, Tessa, Hans, Joop, Jenny, Thibault, Helen, Keiko, and Gonzalo, for being around and making the working days fun!

My dear colleagues at FSE, we are a small group and many of you are always on the road. However, seeing you was always nice and I enjoyed the activities we did together, like the yearly New Year's dinner, WE-day and spontaneous dinners. I love your idealism, especially when it comes to making the world's agriculture more sustainable, more environmentally friendly and more fair! Yodit, thank you for all our talks about the unnecessary complexity of statistical analyses and the meaning of our PhD's and careers. Lieneke, I'm glad I had the opportunity to go outside a bit while stuck behind the computer, whether it was the time I accompanied you to your field work or when we went wadlopen. Mirja, I will never forget your small acts of kindness throughout my $\mathrm{PhD}$. Walter en Kees, bedankt voor de gezellige tijd bij de Farm Design excursie! Wampie en ook Gemma, bedankt voor jullie hulp met administratieve zaken en de levendigheid op de groep. Rogier, wat leuk dat ik af en toe wat grafisch werk kon doen voor de groep! Also thanks to all the others; Francine, Carl, Roos, Victoria, Lenora, Géorges, Hennie, Uma, Bas, Erika, Dirk, Jeroen, Pablo and Daniel.

Finishing this $\mathrm{PhD}$ would also not have been possible without the occasional time for having fun with my friends! Brian, toen wij elkaar als eersteklassers ontmoetten dachten we echt niet dat we 17 jaar later (nog) vrienden zouden zijn, maar daar zijn we dan. Ook José en Jolein bedankt voor jullie steun en gezellige 
gesprekken! Wytze, Denys, Madeleine en Pim, we hebben de afgelopen jaren vele avonden doorgebracht aan een eettafel overladen met papieren, boeken, dobbelstenen en snacks, en al heel wat karakters versleten samen! Ruben, Quirijn en Carel, leuk dat we regelmatig bordspelletjes konden spelen, en het was fijn om met mensen buiten je directe kring te kunnen klagen over PhDzaken. Pascal en Joseline, jullie waren superburen!

Dan wil ik graag mijn familie bedanken. Gem en Lot, jullie zijn de liefste zusjes! Ondanks dat jullie allebei insecten echt he-le-maal niks vinden, weten jullie toch soms een beetje mee te doen met mijn enthousiasme wanneer ik een leuke foto heb gemaakt of wat heb geschreven. Opa en Omi, altijd als we bij jullie in Frankrijk vakantie kwamen houden, zag ik de meest fantastische beesten in de tuin. Opa, het is zeer bewonderenswaardig hoe graag jij kennis vergaart, ook nog op de leeftijd die je nu hebt! Jij bent voor mij een groot voorbeeld om altijd te blijven leren.

Mama en papa, bedankt voor de dingen die jullie mij hebben bijgebracht. Mama, van jou leerde ik creatief te zijn en oog te hebben voor de mooie wereld en mensen om mij heen. Papa, van jou leerde ik een kritische houding te hebben en om altijd hard te blijven werken, en vooral om geduld te hebben.

Mijn lief, Jaap, dank je wel voor alles de afgelopen jaren. Waar was ik toch zonder jou? Je hebt tijdens mijn eerste drukke veldseizoen de kleine kitten Watson opgevoed (“hij is een ramp geworden!”). Ik kan bij jou ook altijd mijn enthousiasme over insecten kwijt en je helpt ook met mijn vage projectjes, zoals op late avonden zwermen eendagsvliegen fotograferen ("aaaah, ik vind dit niet meer leuk, ze zitten overal op me!!”) of mijn flitser, reflectiescherm en acrylplaten vasthouden bij een klein bloemetje. Je weet ook de verrassingen in huis altijd te tolereren ("Yavan, waarom lopen er rupsen over de gordijnen?" - “je bidsprinkhaan is weer ontsnapt!”). Bij jou ben ik altijd thuis en ik ben benieuwd waar wij samen nog allemaal gaan komen. 

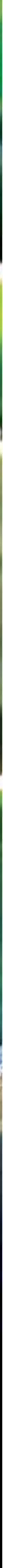


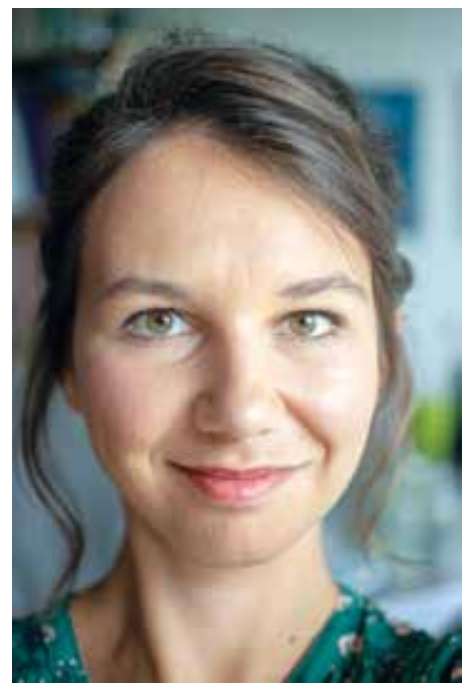

\section{About the author}

Yavanna Sietske Yoka Aartsma was born on 31 March 1989 in Zoetermeer, the Netherlands, but lived in the nearby city of Voorburg for most of her childhood. After completing secondary education in the Hague in 2007, she enrolled in the BSc Biology programme at Wageningen University. During her studies, she was an active member of the study association B.V.W. 'Biologica' as member of the board, chair of the photo committee and author/designer of the periodical Missing Link and two yearbooks.

After obtaining her BSc degree, Yavanna continued with the MSc Biology with a specialisation in Bio-interactions. For her MSc thesis, Yavanna wanted to do entomological field work. She studied pollinator and predatory wasp behaviour at the laboratory of Entomology. Later she went to Zürich, Switzerland, to study choice behaviour of cabbage butterflies to either pollinate or oviposit on Brassica rapa plants. After returning to Wageningen and obtaining the MSc degree, she started in 2014 with her $\mathrm{PhD}$ project on the role of herbivore-induced plant volatiles in tritrophic interactions at different spatial scales. The results of this work are presented in this book.

In her free time, Yavanna enjoys being outdoors and photographing nature. All photos in this thesis are made by her. 


\section{Publication list}

\section{Articles in refereed journals}

Aartsma Y., Leroy B., van der Werf W., Dicke M., Poelman E.H., Bianchi F.J.J.A.. 2018. Intraspecific variation in herbivore-induced plant volatiles influences the spatial range of plant-parasitoid interactions. Accepted for publication in Oikos, doi:10.1111/oik.05151

Aartsma Y., Bianchi F.J.J.A., van der Werf W., Poelman E.H., Dicke M. 2017. Herbivoreinduced plant volatiles and tritrophic interactions across spatial scales. New Phytologist 216(4): 1054-1063.

Lucas-Barbosa D., Dicke M., Kranenburg T., Aartsma Y., van Beek T.A., Huigens M.E., van Loon J.J.A.. 2016. Endure and call for help: strategies of black mustard plants to deal with a specialized caterpillar. Functional Ecology 31: 325-333

Lucas-Barbosa D., Poelman E.H., Aartsma Y., Snoeren T.A., van Loon J.J.A., Dicke M. 2014. Caught between parasitoids and predators - Survival of a specialist herbivore on leaves and flowers of mustard plants. Journal of Chemical Ecology 40(6): 621-631.

\section{Submitted}

Aartsma Y., Pappagallo S., van der Werf W., Dicke M., Bianchi F.J.J.A., Poelman E.H., Plant traits interact with habitat context in determining the strength of host-parasitoid relationships.

Aartsma Y., Calvo Agudo M., Vogel C., Villela A., Etalo D.W., van der Werf W., Dicke M., Bianchi F.J.J.A., Poelman E.H., Herbivory by hosts and non-hosts, in interaction with plant traits, affects herbivore-induced plant volatiles and foraging efficiency of a specialist parasitoid.

Aartsma Y., Hao Y., Dicke M., van der Werf W., Poelman E.H., Bianchi F.J.J.A., Variation in herbivore-induced plant volatiles and alternative host plant cover mediate tritrophic interactions at the landscape scale.

Aartsma Y., Cusumano A., Fernández de Bobadilla M., Rusman Q., Vosteen I., Poelman E.H., Understanding insect foraging in complex habitats by comparing trophic levels. 


\section{PE\&RC Training and Education Statement}

With the training and education activities listed below the $\mathrm{PhD}$ candidate has complied with the requirements set by the C.T. de Wit Graduate School for Production Ecology and Resource Conservation (PE\&RC) which comprises of a minimum total of 32 ECTS (= 22 weeks of activities)

\section{Review of literature (6 ECTS)}

- Herbivore-induced plant volatiles and tritrophic interactions across spatial scales

\section{Writing of project proposal}

- Linking local and landscape scale trophic interactions for plant-induced biological control of insect pests (2014)

\section{Post-graduate courses (3.6 ECTS)}

- Spatial ecology; PE\&RC (2014)

- Bugs at your service; PE\&RC (2014)

- Linear mixed models; PE\&RC (2016)

\section{Invited review of (unpublished) journal manuscript (3 ECTS)}

- Arthropod-Plant Interactions: herbivore-induced plant volatiles and plantpredator interactions (2016)

- Arthropod-Plant Interactions: effects of plant quality on the second and third trophic level (2017)

- Ecological Entomology: indirect defence via induced plant volatiles (2018)

\section{Deficiency, refresh, brush-up courses (6 ECTS)}

- Systems analysis, simulation and systems management; Wageningen University PPS (2014)

\section{Competence strengthening / skills courses (3.3 ECTS)}

- Competence assessment; WGS (2014)

- Techniques for writing and presenting a scientific paper; WGS (2015)

- PhD Workshop carousel; WGS (2015)

- Career orientation; WGS (2016)

- Last stretch of the PhD programme; PE\&RC (2016) 


\section{PE\&RC Annual meetings, seminars and the PE\&RC weekend (3 ECTS)}

- First Year PE\&RC Weekend (2014)

- Middle PE\&RC Weekend (2015)

- PE\&RC Day $(2015,2016,2017)$

- Last Year PE\&RC Weekend (2017)

\section{Discussion groups / local seminars / other scientific meetings (21.1 ECTS)}

- Insect-Plant-Interactions discussion group (2014-2017)

- Entomology PhD discussion group (2014-2017)

- Netherlands Annual Ecology Meeting (2015)

- R Users meeting (2015-2016)

- Netherlands Annual Ecology Meeting; poster presentation (2016)

- Wageningen PhD council PhD symposium; oral presentation (2016)

- NEV Entomologendag; poster presentation (2017)

- Netherlands Annual Ecology Meeting; oral presentation (2018)

\section{International symposia, workshops and conferences (7.2 ECTS)}

- Netherlands-Japan seminar on parasitoid biology; poster presentation; Wageningen, the Netherlands (2014)

- International entomophageous insects conference; oral presentation; Torre del Mar, Spain (2015)

- Symposium on insect plant interactions; oral presentation; Tours, France (2017)

\section{Lecturing / Supervision of practicals / tutorials (13.2 ECTS)}

- Systems analysis, simulation and systems management (2015)

- Insect-plant interactions (2015)

- Insect ecology $(2015,2016,2017)$

- Analysis and design of organic farming systems (2017)

\section{Supervision of MSc students (12 ECTS)}

- Spatial and temporal implications of patch searching behaviour in the parasitoid Cotesia glomerata in a complex odorous environment

- Response of a natural herbivore community to neighbouring wild and domestic brassicaceous species (Brassica nigra L. and Brassica oleracea var. alba L.)

- Preference and performance of Cotesia glomerata on wild vs cultivated plant species: the effect of flowering and cultivar attractiveness

- Effect of landscape composition on parasitism rate of Pieris brassicae on different cabbage cultivars 
The research described in this thesis was supported by the Division for Earth and Life Sciences (ALW) with financial aid from the Netherlands Organization for Scientific Research (NWO).

Cover design, layout and photography by:

Yavanna Aartsma

Printed by:

GVO drukkers \& vormgevers, Ede, The Netherlands 
